


\section{Homme et animal, la question des frontières}

Valérie Camos, Frank Cézilly, Pierre Guenancia et Jean-Pierre Sylvestre, coordinateurs

Éditions Quæ

c/o Inra, RD 10, F - 78026 Versailles Cedex 


\section{Collection Update Sciences \& Technologies}

Le golfe du Lion. Un observatoire de l'environnement en Méditerranée André Monaco, Wolfgang Ludwig, Mireille Provansal, Bernard Picon, coordinateurs 2009, 384 p.

Politiques agricoles et territoires

Francis Aubert, Vincent Piveteau, Bertrand Schmitt, coordinateurs 2009, 224 p.

La mise à l'épreuve. Le transfert des connaissances scientifiques en questions Christophe Albaladejo, Philippe Geslin, Danièle Magda, Pascal Salembier, coordinateurs 2009, 280 p.

Contaminations métalliques des agrosystèmes et écosystèmes péri-industriels Philippe Cambier, Christian Schvartz, Folkert van Oort, coordinateurs 2009, 308 p.

Conceptual basis, formalisations and parameterization of the STICS crop model Nadine Brisson, Marie Launay, Bruno Mary, Nicolas Beaudoin, editors 2008, 304 p.

Le code de la propriété intellectuelle interdit la photocopie à usage collectif sans autorisation des ayants droit. Le non-respect de cette disposition met en danger l'édition, notamment scientifique, et est sanctionné pénalement. Toute reproduction, même partielle, du présent ouvrage est interdite sans autorisation du Centre français d'exploitation du droit de copie (CFC), 20 rue des Grands-Augustins, Paris 6e. 


\section{Sommaire}

Présentation

Chapitre 1. L'écologie évolutive et la distinction homme-animal

Bernard Brun

Chapitre 2. La monogamie humaine est-elle « naturelle"?

Frank Cézilly

Chapitre 3. Les fonctions cognitives élémentaires chez l'enfant et l'animal

Valérie Camos et Jacques Vauclair

Chapitre 4. Brève histoire des idées et représentations des monstres hybrides entre l'homme et l'animal

Pierre Ancet

Chapitre 5. Quelques doutes sur la différence entre l'homme et l'animal

Pierre Guenancia

Chapitre 6. L'animal comme miroir de l'homme au XVIII ${ }^{\mathrm{e}}$ siècle

Jean-Luc Guichet

Chapitre 7. Humanité et animalité dans la pensée humaniste..

Jean-Pierre Sylvestre

Chapitre 8. La construction des mondes animaux et du monde humain selon Jacob von Uexküll 
Chapitre 9. La morale au royaume des animaux.

Catherine Larrère et Raphaël Larrère

Chapitre 10. Éthique et éthologie : comportement animal et conduite humaine Jean-Philippe Pierron

Chapitre 11. Les propositions d'extraction des animaux de la catégorie des objets de droit Sonia Desmoulin

Chapitre 12. Culture de l'élevage et barbarie des productions animales

Jocelyne Porcher

Chapitre 13. Des frontières familières à l'épreuve

d'une expérimentation de contraception animale.

L'" affaire " des marmottes de Prapic

Isabelle Mauz et Céline Granjou

Chapitre 14. Espoirs et désespoirs de l'extrapolation des résultats expérimentaux

Luc Rochette

Bibliographie

Liste des auteurs 


\title{
Présentation
}

\author{
JEAN-PIERRE SyLVESTRE
}

L'animalité - autrement dit, l'ensemble constitué de toutes les espèces vivantes différentes de l'espèce humaine - est rarement interrogée et explorée de manière explicite. Elle est pourtant source de perplexités bien aussi profondes que la question de l'essence de l'homme, qu'elle contribue d'ailleurs à éclairer et à infléchir si l'on veut bien considérer que l'animalité n'est sans doute pas une et que la connaissance approfondie de sa diversité peut conduire à bousculer l'apparente sûreté et stabilité des frontières établies avec l'humanité par les traditions théologiques et philosophiques dominantes. À l'évidence, l'animal et l'homme ont en commun - outre la naissance, la vie et la mort -, la faim, la soif, le plaisir, la peur, la douleur. Les oppositions classiquement reconnues comme significatives se sont concentrées sur le corps et l'âme, l'instinct et la raison, le cri et la parole, la nécessité et la liberté. Or on peut se demander si aujourd'hui elles ne sont pas dépassées par le progrès des connaissances scientifiques relatives notamment à l'écologie comportementale, à l'intelligence animale et aux formes complexes de la vie sociale chez les espèces supérieures. Une chose est certaine en tout cas, c'est l'influence des considérations ou des présupposés axiologiques sur les conceptions respectives de l'humanité et de l'animalité.

Dans l'univers occidental du moins, les pensées les plus communes comme les plus spéculativement élaborées emploient en fait le terme " animalité » en deux sens différents. D'une part, l'animalité est l'ensemble des qualités ou facultés des êtres composant le règne animal; de ce point de vue, biologique et évolutionniste, les êtres humains appartiennent indiscutablement à ce règne. D’autre part, le terme désigne la nature, la condition et le statut des animaux non humains; dans cette perspective, qui est privative, l'animalité est un moins être que l'humanité, située au sommet de la hiérarchie des « étants », parce que «plus riche en monde» pour paraphraser Heidegger ${ }^{1}$. Privés de

\footnotetext{
${ }^{1}$ Martin Heidegger (1992, p. 361) considère que l'animal, contrairement à la pierre par exemple, a bien un monde, au sens d'un environnement qu'il utilise sélectivement, mais que seul l'homme est configurateur de monde. Certes, il existe bien une pluralité des mondes animaux, comme le montrent les travaux de von Uexküll, mais celle-ci s'efface devant un trait commun qui est le « retrait de toute possibilité de percevoir quelque chose comme étant quelque chose », propriétésupposant la pensée réflexive et la capacité de se distancier de la vitalité essentiellement pulsionnelle.
} 
pensée réflexive, et par conséquent d'autonomie véritable, même les animaux les plus proches de l'homme, comme les grands singes, ne peuvent accéder à la dignité de sujets moraux et de sujets juridiques, ce qui ne leur interdit cependant pas d'avoir des droits et d'inspirer aux hommes des devoirs à leur égard.

Nous sommes au cœur d'un paradoxe, semble-t-il. En effet, scientifiquement, l'unique hypothèse explicative sérieuse dont nous disposions actuellement concernant la provenance et la nature de l'être humain est proposée par la biologie de l'évolution, et il s'agit d'une hypothèse continuiste. Mais il se trouve qu'elle est élaborée au sein de la modernité occidentale qui, aux plans philosophique et éthique, reste très attachée à la thèse de l'exception humaine ${ }^{2}$. Celle-ci repose sur la conjonction de quatre affirmations réservant l'esprit au seul être humain à l'exclusion des autres classes d'êtres.

- Il existe une différence de nature entre l'homme et tous les autres êtres vivants. L'enjeu ne concerne pas une simple propriété spécifique de l'être humain (le langage par exemple), en vertu de laquelle il se distinguerait des autres êtres vivants, euxmêmes différenciés en espèces et sous-espèces aux caractéristiques propres ; il porte sur l'absolue singularité de l'humanité, et l'affirmation que seul l'homme transcende l'ordre biologique, auquel il appartient pourtant en tant qu'être vivant, par sa capacité spirituelle à s'instituer lui-même comme sujet.

- La thèse de l'exception humaine n'oppose pas seulement deux domaines du vivant, celui de l'humain et celui de l'animalité. Elle s'exprime également dans la conception de l'homme lui-même à travers un ensemble d'oppositions binaires : corps/âme, affectivité/ rationalité, instinct/moralité, nécessité/liberté, nature/culture... Davantage que l'opposition explicite entre l'être humain et l'être animal, ce sont sans doute ces dichotomies internes à la définition de l'homme qui sont constitutives de notre vision du monde. En effet, dans la plupart des sociétés, il est rare que l'être humain soit considéré comme possédant des qualités ou propriétés ne se retrouvant chez aucun autre être vivant ${ }^{3}$.

- Ce qui est exclusivement humain dans l'homme, c'est l'autoconnaissance qui rend possible l'autonomie. Dans la version théologique et chrétienne de cette thèse, seul l'homme est capable de se connaître et de connaître (en partie du moins) son créateur puisqu'il est à son image. Dans ses versions « laïcisées », dont le cartésianisme est sans doute la figure inaugurale, l'homme est en mesure, grâce à la raison, secondée par la volonté, de conduire librement sa vie.

- Le caractère incommensurable de l'être humain exige une voie d'accès qui se distingue de celle permettant d'appréhender la nature inanimée et les autres êtres vivants. Cette affirmation impose un idéal cognitif antinaturaliste, c'est-à-dire non seulement antibiologiste, mais aussi bien antipsychologiste ou antisociologiste puisqu'il ne peut être question de comprendre le fondement des actions humaines en adoptant un point de vue exclusivement externe.

En même temps qu'elle interdit de concevoir le rapport d'homme à homme autrement qu'un rapport de sujet à sujet, la thèse de l'exception humaine a pour conséquence essentielle de conduire à penser le rapport de l'homme au monde non humain comme un rapport de sujet à objet. Parce que l'homme seul est sujet de sa pensée et de son action, parce qu'il s'autoconstitue simultanément comme conscience de soi et conscience du

\footnotetext{
${ }^{2}$ Pour une analyse approfondie et critique de cette thèse, $c f$. Jean-Marie Schaeffer, 2007, chapitre premier.

${ }^{3}$ Sur cette question, $c f$. Philippe. Descola, 2005.
} 
monde, il est l'instance à partir de laquelle tout ce qui n'est pas lui se trouve institué en objectivité pure et par conséquent disponible pour toutes les formes d'utilisation et de manipulation décidées par lui. Une telle représentation de la subjectivité induit donc ce que l'on appelle souvent le «prométhéisme » de la modernité occidentale, c'est-à-dire son attitude conquérante à l'égard de la nature et, corrélativement, sa propension à faire de l'homme le maître et l'artisan de son existence individuelle et collective.

En somme, la thèse de l'exception humaine est d'abord conduite à tracer une frontière de principe entre sa manière propre d'aborder l'esprit humain et les recherches menées par les sciences expérimentales ou sociales « objectivistes ». Ensuite, et surtout, elle ne peut légitimer cette frontière qu'en affirmant que le propre de l'homme est en lui ce qui échappe à toute étude " externaliste », c'est-à-dire à toute étude l'abordant comme un objet du monde animé parmi d'autres objets. Ainsi, le privilège épistémique accordé à l'autoconstitution de la conscience, comme fondement « internaliste » de toute validité cognitive, et l'humanisme antinaturaliste s'impliquent-ils réciproquement en tant qu'ils constituent les deux faces de la figure moderne de la thèse de la singularité humaine affirmant l'autonomie de l'activité consciente en tant que conscience de soi. En effet, la conscience ne peut s'assurer de sa propre identité que si elle pose une extériorité que précisément elle n'est pas : celle du monde, des objets et donc des corps biologiques, par opposition au «corps propre », c'est-à-dire au corps subjectif ou corps «pour soi » qu'est le corps humain, irréductible à sa seule matérialité ${ }^{4}$.

La thèse de l'irréductibilité de l'esprit à l'étude d'un ensemble de propriétés caractérisant certains types d'organes biologiques et, par extension, le rejet des études « externalistes » ou objectivistes ayant la prétention d'élucider les faits mentaux (écologie comportementale, éthologie, neurobiologie, psychologie cognitive...) sont donc la conséquence du postulat fondant la connaissance de l'homme sur l'auto-compréhension de la conscience, faisant par là même de l'homme un être pensant transcendant la loi commune du vivant, et donc séparé par essence de l'ordre de l'animalité. Or deux grands types de critique convergent aujourd'hui pour remettre en question la thèse de la singularité radicale de l'homme.

$\mathrm{Au}$ plan scientifique d'abord, de nombreux travaux de primatologues tendent à mettre en évidence que, du point de vue de l'identité psychologique (conscience de soi, empathie, coopération, "gestion » des conflits... $)^{5}$ comme de celui des performances cognitives, la différence entre les grands singes et l'homme ne serait pas de nature mais seulement de degrés. D’une manière plus générale, la biologie de l'évolution ne nie pas qu'il existe des différences majeures entre l'homme et les animaux, mêmes supérieurs, mais elle les réintroduit dans un paradigme explicatif qui est « continuiste », évolutionniste et non anthropocentré. La distinction entre l'homme et les autres animaux peut être

\footnotetext{
${ }^{4}$ Rappelons que, pour la phénoménologie, ce qui distingue le «corps propre » du corps biologique, objet d'observation anatomique, d'expérimentation et de manipulation, c'est qu'il ne peut être considéré comme une chose ou un objet physique auquel la conscience viendrait s'ajouter. Il se présente au contraire comme la manière spécifique dont l'homme accède au monde et, en même temps, comme un mode d'opposition au monde. Tandis que les animaux sont enfermés dans leurs comportements, captés par des données de leur milieu (en raison de déclencheurs innés ou acquis) et donc incapables d'opérer une "suspension » de leurs comportements, l'être humain, en revanche, n'est pas strictement enfermé et capté car son corps est plus qu'un organisme animal : il n'est pas pris dans un milieu naturel, mais « inscrit » dans un monde. Autrement dit, le corps humain est toujours « spiritualisé ».

${ }^{5} \mathrm{Cf}$. les travaux de Frans De Waal, notamment.
} 
maintenue, non pas tant comme une différence entre nature et culture, mais comme un ensemble de différences dans la nature, autrement dit sans remise en question de l'unité biologique substantielle entre tous les êtres. Ainsi, l'espèce humaine a évolué en développant des dispositions, linguistiques, sociales, culturelles, axiologiques et juridiques qui n'existent pas chez les autres espèces animales, ou alors seulement à l'état d'ébauche. Ces dispositions n'en sont pas pour autant antinaturelles. Elles doivent être considérées comme des séquences historiques dans l'évolution du vivant. Selon cette thèse, l'humanité de l'homme n'est pas une autofondation arrachée à la composante biologique de sa nature, mais la cristallisation, au cours de sa généalogie phylogénétique et de son histoire, de structures mentales et d'aptitudes particulières. La culture demeure un fait biologique qu'il convient d'expliquer comme un niveau organisationnel et fonctionnel propre.

Au plan éthique, ensuite, des formes inédites d'appropriation des animaux vivants (élevage « en batterie », expérimentation, transgénose) ont conduit à une instrumentalisation accrue de ceux-ci. Sans intention délibérée de nuire, mais par ignorance et sous la pression des intérêts économiques, les hommes infligent aux animaux des traitements qui vont à l'encontre de leur bien-être minimum. À cela s'ajoutent les menaces pesant sur les espèces sauvages en raison du développement des sociétés industrielles. Certains analystes ou militants de la «libération animale » en viennent ainsi à considérer qu'il existe une véritable solidarité entre les formes de vie humaines et animales qui doit se traduire par une reconsidération en profondeur des normes morales ou juridiques qui régentent et régulent l'ensemble des relations entre humains et non-humains.

Dans cette perspective, il est pertinent d'affirmer, à la fois contre la pensée humaniste et contre celle de Heidegger ${ }^{6}$, en s'inspirant notamment des recherches en éthologie et en sciences cognitives, que les animaux les plus développés ont une " valeur inhérente » en tant qu'ils sont « sujets de leur vie » (Regan, 1983, p. 248), c'est-à-dire qu'ils peuvent conduire leur existence à partir d'une expérience propre et en éprouver le bien être, bref qu'ils ont une authentique vie mentale, fût-elle élémentaire. Il n'est d'ailleurs pas impératif de traiter les animaux comme des êtres humains, mais seulement de prendre en considération leurs intérêts au même titre que ceux des humains, selon un point de vue qui est celui de la philosophie utilitariste (Singer, 1993).

Dans le cas des animaux sauvages, il ne s'agit plus de l'intérêt des animaux, pris comme individus, mais des espèces en tant qu'elles sont constitutives de la diversité, de la richesse, de l'équilibre, mais aussi de la beauté de notre planète et ne peuvent ainsi être instrumentalisées au seul profit des valeurs économiques propres à l'homme. Il n'est pas impératif de soutenir que la nature sauvage nous donne des exemples à suivre, mais de rappeler que toutes les valeurs ne sont pas économiques et que la domesticité n'est pas le destin obligé de l'animalité?

\footnotetext{
${ }^{6}$ La pensée de ce dernier est antisubjectiviste, mais pas pour autant anti « exceptionnaliste », dans la mesure où elle considère que seul l'homme est capable de poser la question de l'être en tant qu'être et, par conséquent, d'accéder à l'existence « authentique » du Dasein, à l'opposé de celle du « on », banale et vulgaire.

${ }^{7}$ En suivant Jean-Yves Goffi (2004, p. 27), il convient de distinguer au sein du règne animal les animaux domestiques et les animaux sauvages, car n'ayant pas la même vie, ils ne sont pas également menacés par les conséquences de l'activité humaine qui les concernent directement ou indirectement : « En un mot, ce qui est menacé dans le cas des animaux domestiques, c'est leur animalité, en tant qu'elle est suffisamment individualisée pour évoquer notre humanité ; dans le cas des animaux sauvages, c'est leur sauvagerie, en tant qu'elle est assez intacte pour évoquer la nature pristine. Les enjeux philosophiques ne sont pas les mêmes dans les deux cas ».
} 
Cependant, même si l'on reconnaît que d'autres espèces animales ont développé des formes rudimentaires de culture et de moralité, l'homme ne reste-t-il pas l'animal culturel par excellence, grâce au caractère accumulateur et autocatalytique de ses capacités d'apprentissage, à ses aptitudes langagières et au rôle incomparable joué par son imaginaire instituant? Ces caractères et capacités propres ne sont-ils pas la clé interprétative de ses performances cognitives et réflexives, puisqu'ils lui confèrent une autonomie dans la conduite de sa vie individuelle et collective que l'on ne saurait retrouver chez aucun autre animal ? Plus encore, l'homme n'est il pas également et fondamentalement un animal métaphysique et un animal éthique ? N'est-il pas en effet le seul à poser explicitement la question de son essence ; celle aussi de la finalité de son existence et de toute existence ; celle enfin de son rapport à autrui (l'autre homme, l'autre animal et l'autre en général), non seulement en termes de connaissance, mais tout autant en termes de reconnaissance et de responsabilité pleinement assumées?

Les textes rassemblés dans cet ouvrage ne proposent pas de réponses univoques à l'ensemble des problèmes posés : elles varient selon les prédispositions interprétatives de la discipline d'appartenance des auteurs ou selon leurs convictions et engagements intellectuels. L'objectif visé n'est pas d'atteindre l'unité doctrinale, mais de faciliter aussi bien la clarification des oppositions irréductibles que la levée des incompréhensions ou des malentendus. Corrélativement, il s'agit d'associer et d'articuler, dans la mesure du possible, des travaux d'inspiration prioritairement spéculative ou épistémologique, avec des travaux plus centrés sur des investigations empiriques.

Le manuscrit de cet ouvrage a bénéficié de la lecture critique et des suggestions de Pierre Barrouillet, Jean-Claude Beaune, Michèle Dion et Anne Veynante. Nous tenons à les en remercier vivement. 



\title{
Chapitre 1
}

\section{L'écologie évolutive et la distinction homme-animal}

\author{
BERNARD BRUN
}

L'histoire de la vie sur la terre a été marquée par un flux incessant de changements affectant la répartition des océans et des continents, le climat et la composition de l'atmosphère, le nombre et la nature des espèces vivantes. Plus de $99 \%$ des espèces ayant peuplé la planète ont disparu, mais ces disparitions n'ont pas empêché un accroissement - certes irrégulier et marqué par des crises d'extinction - du nombre total d'espèces, et c'est à tout moment que des espèces s'éteignent tandis que d'autres se transforment, à des rythmes très variables d'ailleurs, et enfin que de nouvelles espèces apparaissent.

Les relations qu'entretiennent les espèces vivantes avec leur milieu ne cessent donc de changer au cours de l'histoire de la biosphère, et ce n'est que si nous les observons sur un laps de temps réduit qu'elles peuvent nous apparaître immuables ${ }^{1}$. L'écologie évolutive a pour objet de décrire et d'interpréter ces changements. Elle s'inscrit donc dans le cadre plus vaste de la biologie de l'évolution mais, contrairement à d'autres sous-disciplines telles que l'anatomie comparée ou la systématique, elle se focalise sur l'analyse des modalités de changements dans la relation espèce/milieu dans le temps même où ils s'effectuent plutôt que sur leur résultante à long terme. Or, s'interroger sur la distinction homme/ animal semble signifier vouloir d'abord réfléchir sur le bilan d'une longue séparation.

Mais en fait, même lorsqu'elles restent très floues, et qu'elles aient ou non une prétention scientifique, les représentations de ce qui nous distingue des animaux ainsi que la valeur attribuée aux différences sont difficilement dissociables de nos représentations de l'origine de l'humanité, et ce, bien qu'il n'y ait jamais de relation strictement univoque entre telle théorie sur l'origine de l'humanité et tel regard sur ce qui fonderait l'humanité

\footnotetext{
${ }^{1}$ La découverte de momies d'animaux conservées dans des sépultures de l'Egypte ancienne avait semblé un témoignage remarquable de la fixité des espèces, mais que sont les quelques milliers d'années qui nous en séparent en regard ne serait-ce que de la durée de l'ère quaternaire ?
} 
dans sa différence. En raison de cette intrication entre représentation des origines et représentation des différences, on peut espérer que la réflexion sur les modalités de l'évolution puisse en retour éclairer certains aspects de la question de la distinction homme/animal.

\section{Le modèle général de l'écologie évolutive : sélection naturelle et dérive génétique}

Le modèle général de transformation évolutive que propose l'écologie évolutive est celui du changement progressif de la composition génétique d'une population, notamment sous l'effet de la sélection naturelle, mais pas seulement, puisqu'il reconnaît en particulier la possibilité de changements dus à de simples phénomènes d'échantillonnage aléatoire des génotypes et des gènes participant à la reproduction, changements particulièrement importants dans des populations aux effectifs réduits, et connus sous le nom de « dérive génétique $»^{2}$.

L'écologie évolutive s'inscrit sans restriction dans la théorie néodarwiniste de l'évolution et, recourant aux apports de la génétique des populations, ne s'en distingue que par le fait qu'elle ne perd jamais de vue la question de l'inscription des populations dans la complexité vivante des écosystèmes tandis que la génétique des populations s'évade volontiers dans les formalismes mathématiques de modèles dont le degré de réalisme n'est pas toujours facile à apprécier.

Ici, une précision à l'intention des lecteurs francophones n'est sans doute pas superflue : comme le soulignent Gouyon, Henry et Arnould (1997) dans l'avant-propos de leur ouvrage ${ }^{3}$ « ...la résistance des universitaires français à la génétique a longtemps été farouche... » et encore plus, pourrait-on ajouter, la résistance à la théorie néodarwiniste de l'évolution. Pourtant celle-ci recueille selon eux un « consensus quasi unanime »; ils précisent encore que les « ...raisons à cette grave lacune relèvent surtout de l'histoire et de la sociologie $»$.

Concernant l'applicabilité de la théorie néodarwiniste à l'interprétation de l'évolution humaine, les oppositions se sont cristallisées sur le postulat anti-darwinien d'une discontinuité évolutive fondatrice de l'humanité et sur celui de l'indépendance de la culture à l'égard de tout déterminisme biologique ${ }^{4}$. Tandis que divers paléontologistes

\footnotetext{
${ }^{2}$ Le rôle de la dérive génétique, reste largement méconnu des non-spécialistes et a été certainement sous-estimé dans la version standard de la théorie synthétique (néodarwiniste) de l'évolution, car ce qui nous fascine le plus dans le monde vivant, ce sont les faits d'adaptation. Or c'est la sélection naturelle qui en rend compte, même s'il est vrai qu'elle interfère avec le jeu de la dérive génétique.

${ }^{3}$ L'intérêt de l'ouvrage est de faire une large place à l'histoire des théories et de joindre des réflexions épistémologiques à la présentation de la théorie néodarwinienne de l'évolution.

${ }^{4}$ L'anthropologue américain M. Sahlins a formulé ces points de vue avec une clarté et une vigueur particulières dans sa Critique de la sociobiologie. Aspects anthropologiques, 1976 : «Le fil du raisonnement sociobiologique qui, partant de l'évolution phylogénétique, arrive à la morphologie sociale, est rompu par la culture ». Premier ouvrage de critique de la sociobiologie publié peu après l'ouvrage fondateur de E.O. Wilson, il reste un témoignage de l'incompréhension de l'ancrage de la sociobiologie dans la théorie moderne de l'évolution, incompréhension découlant de la croyance en une discontinuité culture/nature fondatrice de l'humanité. Son analyse, comme toutes celles qui tentent de réduire la sociobiologie à une simple idéologie, méconnaît le fait que la sociobiologie, en fait de déterminismes, ne postule que des corrélations - pas nécessairement fortes - entre facteurs génétiques et comportements sociaux, et non un déterminisme mécanique total.
} 
ou biologistes ont cherché une explication biologique d'une prétendue discontinuité (par exemple dans une mutation chromosomique particulière), des anthropologues ou des philosophes l'ont cherchée dans ce qui, à leurs yeux, fonde l'humanité dans sa spécificité unique. Ainsi l'éclosion de la conscience, l'avènement d'un langage comme système clos d'emblée structuré sans correspondances biologiques, ou encore l'interdit de l'inceste ont-ils pu être mis en avant. Ces dernières hypothèses ont le «mérite » - s'il est permis d'ironiser à leur égard - d'évacuer la question de la relation entre l'évolution biologique des ancêtres de l'humanité et les caractères de l'humanité actuelle. Ou bien peut-être présupposaient-elles (mais je n'ai jamais rencontré cette idée explicitement formulée) qu'il eût fallu que la lignée de nos ancêtres ait atteint un certain degré de maturation pour que l'irruption de la culture brise le lien entre la biologie et la culture.

À mon insistance sur le caractère progressif de la transformation des espèces, on pourrait être tenté d'objecter que la théorie néodarwiniste de l'évolution fait pourtant place à la possibilité d'apparition brusque de nouvelles espèces à la suite de mutations chromosomiques de grande ampleur. Mais même lorsque cela s'est produit - comme on l'observe dans certains groupes de poissons ou d'insectes -, le changement qui en résulte est minime et n'est souvent perceptible qu'aux yeux du spécialiste du groupe.

Concernant l'espèce humaine, divers arguments, notamment l'ampleur de la diversité génétique actuelle, s'opposent à l'idée que nos ancêtres aient pu passer par un goulot d'étranglement démographique correspondant à un couple unique. Il faut remarquer à ce propos que la théorie si poétiquement évocatrice d'une Eve africaine serait mal interprétée si l'on supposait que l'humanité ancestrale se soit réduite - côté féminin - à une seule personne. L'Eve africaine de la théorie était membre d'une population au sens habituel du terme. Son existence a été postulée à partir de l'examen de la variabilité génétique de $\mathrm{l}^{\prime} \mathrm{ADN}$ des mitochondries ${ }^{5}$; à partir de $\mathrm{l}^{\prime} \mathrm{ADN}$ du chromosome $\mathrm{Y}$ on remonterait à un Adam appartenant à une autre génération et sans doute une autre population!

Écartant par principe toute discussion sur la signification religieuse et symbolique du couple Adam et Eve de la Bible, j'insisterai par contre sur la nécessité impérieuse de renoncer à cette image dans la réflexion scientifique sur l'évolution humaine. En effet, même si l'on tenait à imaginer un goulot d'étranglement réduit à un couple dans l'histoire des origines de l'humanité (ce que l'écologie évolutive n'exclut pas par principe), on serait placé face à l'alternative suivante : si nous imaginons cet Adam et cette Eve fondateurs très proches dans le temps (100 000 ans par exemple), alors, l'essentiel de ce qui distingue l'humanité et l'animalité actuelles se serait construit progressivement au cours des temps antérieurs. Si à l'opposé nous les imaginons très loin dans le temps (cinq millions d'années par exemple), alors, l'essentiel de la différenciation entre l'animal et l'homme se serait effectué ultérieurement au sein des populations qu'ils auraient engendrées. Si pour échapper à cette alternative, nous émettons une hypothèse intermédiaire, la situation ne sera pas améliorée, puisque nous devrons supposer qu'une fraction de ce qui sépare l'homme et l'animal est antérieure à la création de l'humanité, et une autre postérieure.

Il nous faut donc retourner à la considération du modèle de loin le plus classique de l'écologie évolutive, celui de la transformation progressive de la composition

\footnotetext{
${ }^{5}$ Il s'agit d'organites cytoplasmiques renfermant de l'ADN distinct de celui des chromosomes et transmis seulement en lignée maternelle par les ovules.
} 
génétique d'une population. Plus précisément, puisque ce qui nous intéresse ici est une question de frontières, ce sont les processus de séparation et de divergence à partir d'une espèce ancestrale unique qui vont retenir notre attention. Une période cruciale dans le déroulement de ces processus est celle de la différenciation de deux espèces distinctes à partir d'une population ancestrale unique, ce que l'on désigne sous le nom de « spéciation».

\section{Quelques aspects généraux de la spéciation}

On reconnaît classiquement deux processus principaux d'apparition d'une espèce nouvelle. Le premier consiste en la simple transformation progressive au fil des générations d'une même lignée, jusqu'au point où des paléontologistes ne voient plus dans les fossiles de la population la plus récente la même espèce que celle qu'ils observaient dans les couches anciennes. Dans ce processus, connu sous le nom de « transformation anagénétique » ou " anagenèse », aucune discontinuité majeure n'intervient et, comme le soulignait Darwin, la distinction des espèces - opérée sous cet angle-là - est arbitraire. Le second processus, appelé « cladogenèse », est celui au cours duquel deux populations de la même espèce se séparent, le plus souvent par isolement géographique, puis divergent dans leur composition génétique jusqu'au point où, même si les populations-filles se rencontrent à nouveau, elles ne sont plus susceptibles - au moins dans les conditions naturelles - de fusionner à nouveau. Par définition on dira alors qu'elles constituent deux espèces biologiques distinctes. On dit encore qu'une fois cette situation atteinte, le processus de spéciation est achevé. La distinction entre deux espèces contemporaines, contrairement à celle que l'on opère dans le cas d'un processus d'anagenèse, met en jeu un critère objectif : l'isolement reproducteur des deux entités. Toutefois, si un processus de cladogenèse n'est pas allé jusqu'à un isolement complet, ou encore si l'isolement peut être simplement postulé, mais non démontré, le statut des deux ensembles soumis à examen sera difficile à préciser... et par suite largement tributaire de la subjectivité des auteurs. C'est ainsi que l'homme de Neandertal et l'homme moderne ont été tantôt considérés comme deux sous-espèces d'Homo sapiens, tantôt comme deux espèces distinctes.

Remarquons enfin que, pour que la séparation de deux populations aboutisse à une spéciation cladogénétique, il faut que l'une au moins des deux lignées évolue selon les processus invoqués pour l'anagenèse (sélection naturelle et dérive génétique); mais il serait tout à fait irréaliste de supposer que pendant ce temps l'autre n'évolue strictement pas, et il serait non moins irréaliste de supposer que les deux lignées et les espèces qui en descendront éventuellement évoluent à la même vitesse. Lorsqu'une des deux lignées qui se sont séparées par un processus initial de spéciation engendre à long terme une descendance qui se sera modifiée de façon spectaculaire dans ses caractéristiques morphologiques et écologiques, tandis que l'autre descendance aura moins changé, on a l'habitude de résumer l'histoire globale en évoquant l'origine d'un ensemble taxonomique nouveau à partir d'une souche ancestrale. C'est ainsi par exemple que l'on essaie de dater l'origine des vertébrés tétrapodes à partir des poissons, ou celle des oiseaux à partir des reptiles, et que l'on évoque l'origine de l'humanité à partir d'une souche animale. Mais, comme nous allons le voir, ces expressions sont facilement trompeuses. 


\section{Origines, discontinuités, frontières et distances}

Le Vocabulaire de la philosophie de Lalande rappelle les diverses significations du mot « origine ». Employé au pluriel, il désigne les circonstances qui entourent les commencements d'une entité ( $c f$. Les origines du christianisme), tandis qu'employé au singulier, le terme désigne souvent une première apparition, ou encore le point zéro de l'espace ou du temps à partir duquel peut être mesurée une variable.

La mention d'une origine de l'homme se trouve ainsi inévitablement associée à l'image d'un point zéro, d'une discontinuité radicale qui aurait présidé à la séparation homme/animal. Elle s'associe en conséquence, presque aussi inévitablement, à l'idée d'une dualité culture/nature, qui elle-même renvoie à une dualité âme/corps.

De telles hypothèses - explicitement énoncées ou non - étaient omniprésentes dans les sciences humaines il y a quelques dizaines d'années seulement, souvent d'ailleurs présentées comme la condition (et, sur un mode curieusement défensif, comme la légitimation) de leurs méthodes. Mais en fait, le sentiment d'unicité de l'espèce humaine face au reste du monde vivant imprègne suffisamment la conscience commune pour que même des biologistes avertis ne renoncent pas à évoquer la naissance de l'homme, ou le berceau de l'humanité, expressions qui véhiculent obligatoirement l'image d'un engendrement instantané, qu'on pourrait préciser dans le temps si nous disposions d'archives bien documentées.

C'est ici qu'un retour sur les mécanismes de la spéciation que propose l'écologie évolutive peut aider à dissiper les illusions.

Le premier épisode d'un processus de spéciation repose sur la séparation de deux populations préalablement indistinctes. Cet événement est en lui-même trivial et insignifiant. Il ne pourra acquérir de signification que si cette séparation se maintient suffisamment longtemps pour que les deux lignées divergent jusqu'à constituer deux espèces distinctes. Il est important de souligner qu'au moment même de la première séparation aucun événement évolutif significatif n'intervient; rien, si ce n'est le fait « géographique » de la séparation elle-même ; et rien, au départ ne permet de distinguer significativement les deux lignées.

Ces remarques valent, aussi loin que l'on remonte dans le passé, pour tous les épisodes de spéciation qui ont jalonné l'histoire évolutive de nos ancêtres. Elles valent aussi bien si la lignée souche de nos représentations s'est perpétuée (spéciation qui a séparé la lignée des hominidés de celle des pongidés, toujours représentée par les « grands singes » actuels) que si la lignée souche a disparu (cas de la spéciation qui a dû présider à la différenciation du genre Homo à partir des australopithèques).

Aussi, j'avancerai l'idée de renoncer totalement aussi bien au concept de discontinuité qu'à celui de frontière bien qu'il figure dans le sous-titre du présent ouvrage. Ce dernier terme renvoie en effet également à l'image d'une coupure, d'une ligne de démarcation qui ne peut être qu'arbitraire. Le biologiste de l'évolution ne peut manquer de souligner ici une convergence avec le point de vue de l'ethnologue. Dans, Limites floues, frontières invisibles. Des variations culturelles en France et à l'étranger, (Bromberger et Morel (dir.), 2005) ${ }^{6}$, T. Schippers souligne que « ...vues de près, les frontières culturelles

\footnotetext{
${ }^{6}$ L'intérêt de cet ouvrage collectif déborde beaucoup le seul domaine de l'ethnologie par la qualité des réflexions qu'il apporte sur les questions de frontières et de continuité.
} 
semblent introuvables... » et que « ...le chercheur animé par la meilleure volonté cartographique du monde, en vient à désespérer devant un fouillis qui ne se laisse jamais réduire à une ligne $»$.

Sans doute y a-t-il d'ailleurs, au-delà de la question de l'origine de l'homme, une tendance à une représentation mythique de toute origine, et il est probable que si nous connaissions le film des conditions de l'apparition de la vie, nous serions incapables d'y fixer un instant zéro. Plus près de nous, il est peu vraisemblable qu'une intuition géniale ait pu présider à la découverte du feu : il semble bien plus réaliste de postuler un apprentissage par étapes de l'utilisation des bénéfices secondaires d'incendies naturels.

Pour contrer l'image d'une discontinuité fondatrice, j'avancerai la métaphore de la naissance d'un cours d'eau à partir d'une tourbière comme on peut l'observer fréquemment dans le Massif Central. Le choix d'une métaphore est toujours délicat car une métaphore n'est pas un modèle et ne se prête guère à l'approfondissement ; l'expérience montre d'ailleurs avec quelle facilité elle peut se retourner contre elle-même ${ }^{7}$. Ces réserves énoncées, je vous invite à patauger dans une tourbière d'altitude : le sens du courant dans les flaques d'eau libre entre les touffes de sphaignes est indéterminable pour l'observateur. Mais si l'on chemine vers l'aval de la vallée, ce qui dans la métaphore représente la marche selon l'axe du temps, on verra bientôt de droite et de gauche des suintements d'eau significativement orientés, sans que l'on puisse encore identifier un cours d'eau bien défini. Puis se dessinera plus nettement un ruisseau aux bras multiples et anastomosés. Plus bas encore, il prendra l'image habituelle d'un torrent aux rives bien délimitées, mais encore franchissable d'un seul bond. Bien plus bas, le torrent, devenu fleuve, sera infranchissable à pied. À aucun moment, si ce n'est au prix d'une extrême simplification nous n'aurons pu situer sa source.

Imaginons maintenant une population, disons de préhumains, qui aurait vécu à une certaine période dans l'espace de la tourbière et dont sont issus deux groupes qui très lentement se déplacent au fil des siècles ou des millénaires vers l'aval. Au départ rien ne les distingue. Pendant un temps, les échanges sont encore possibles. Puis ils cessent définitivement et enfin vient le moment où, d'une rive à l'autre, les deux groupes, trop longuement séparés, ne se reconnaissent plus : malgré leurs ressemblances persistantes, la distance qui les sépare est devenue abyssale.

Aux concepts de rupture, discontinuité ou frontière, il est donc préférable de substituer celui de distance. La proposition n'est pas neuve et Lamarck utilisait déjà ce terme pour nous situer à proximité des grands singes dans «l'échelle des êtres ». Mais maintenant que presque rien ne semble devoir résister à l'impératif de la quantification mathématique, se pose la question de la mesure de la distance qui nous sépare des

\footnotetext{
${ }^{7}$ Soutenir qu'aucune discontinuité fondamentale n'a jamais coupé l'humanité de l'animalité conduit à postuler que l'histoire évolutive du développement des capacités intellectuelles chez nos ancêtres a dû reposer essentiellement sur le jeu de la sélection naturelle : le développement de la culture a dû nécessiter une intrication permanente entre tendances et capacités génétiquement programmées et faits d'apprentissage. Pour des raisons idéologiques, de nombreux auteurs récusent la dichotomie entre inné et acquis : pour eux, l'absurdité de la distinction est illustrée par la métaphore du tissage. N'est-il pas aberrant de se demander si la qualité d'un tissu tient plutôt à celle du fil de chaîne ou à celle du fil de trame ? En effet, elle est intrinsèquement liée à leur entrecroisement. Et pourtant... tout industriel du tissage, comme tout tisserand artisan ou amateur sait très bien qu'il n'y a pas les mêmes facilités et difficultés, les mêmes avantages ou inconvénients à modifier fil de chaîne ou fil de trame.
} 
animaux. Tant qu'il s'agit de comparer des groupes animaux entre eux, les biologistes se satisfont aisément de tout un ensemble de techniques d'évaluation présentant chacune ses avantages et ses inconvénients, mais toutes objectivables soit en termes de ressemblances biométriques, soit en termes de ressemblances génétiques. Mais, que nous apporte réellement le fait de savoir que chimpanzés et hommes auraient $99 \%$ de leur information génétique en commun? Et si cet énoncé devait être infirmé et la distance multipliée par deux, qu'est-ce que cela changerait dans notre regard sur les animaux ?

Il est en effet impossible de ne pas attribuer une valeur subjective à la distance qui nous sépare des animaux, ou, si l'on préfère son miroir positif, à la proximité homme/ animal. Mais il n'existe aucune solution scientifique pour substituer ici une mesure rationnelle à une évaluation subjective.

L'impossibilité de passer d'un ordre de la quantification des distances biologiques à un ordre de l'éthique ne justifie pas pour autant la négation d'une distance, et je voudrais manifester ici ma profonde inquiétude devant la multiplication des tentatives de faire des animaux des « sujets de droit», d'étendre aux grands singes les droits de l'homme ou encore de fonder une prétendue « éthique du vivant». Nier la distance qui, au terme actuel d'un long processus évolutif, sépare l'humanité des animaux les plus proches m'apparaît comme nier la spécificité humaine, et par suite contenir en germe la négation d'un principe d'humanité qui veut que nous ne traitions pas nos semblables comme des bêtes.

\section{La construction des différences évolutives : l'évolution en mosaïque}

Lorsqu'un processus de divergence s'enclenche à partir de deux populations appartenant initialement à la même espèce, il tend nécessairement à s'approfondir au cours du temps. Les deux populations filles ne se développant pas dans le même milieu, les forces sélectives qui tendent à les modifier n'agiront pas de la même façon sur l'une et sur l'autre; les différences tendront à s'accentuer car, une fois les premières différences acquises, même des changements extérieurs semblables ne produiront plus les mêmes effets. C'est tout un ensemble de forces sélectives différentes (sans compter les effets de dérive génétique) qui va modeler selon des rythmes différents et inconstants les deux lignées.

Il en résulte que les différences finales entre les deux groupes tiendront plus de l'accumulation d'une somme intriquée de variations distinctes qu'à une divergence assignable à un processus unique, même progressif. Bien sûr, toutes les différences n'ont pas le même " poids » dans le résultat final et ne sont pas pleinement indépendantes les unes des autres. La difficulté de penser l'évolution est justement d'avoir à écarter l'image du « sac de graines », selon l'expression imagée de E. Mayr (1984), où l'individu ne serait que le résultat de l'évolution séparée de chacun des gènes qui composent son génome, mais de devoir écarter également l'idée que l'évolution se ferait selon une tendance assignable à une raison unique qui expliquerait l'ensemble de toutes les variations.

Le sentiment d'unicité de l'espèce humaine a conduit à la tentation de céder à cette dernière facilité : même lorsqu'on abandonne l'idée d'une discontinuité, il est tentant de rechercher un critère unique bien identifié et d'en faire le caractère fondateur. 
Paradoxalement, cette tentation est d'autant plus grande que l'on regarde les choses de plus loin, car alors la multiplicité des particularités tend à s'effacer devant la prise en considération d'une différence globale prédominante.

Les paléontologistes qui s'attachent à un problème étroitement circonscrit tel que la caractérisation des hommes modernes à partir d'éléments crâniens fossiles, question bien plus limitée que celle de la distinction homme/animal, renoncent à l'identification d'un critère unique.

«Ce qui caractérise les hommes modernes, écrit B. Vandermeersch ${ }^{8}$ dans un article récent, c'est l'association d'une forme crânienne arrondie, d'un grand volume cérébral, d'une diminution marquée de la robustesse... ». Il ajoute encore d'autres caractères et précise : «...l'évolution est un processus en mosaïque, les différentes parties du crâne n’ont pas évolué au même rythme dans les différentes populations ».

Ce que Vandermeersch affirme sur la base de l'examen de restes fossiles peut être postulé pour tout caractère synthétique, estimé trop souvent unitaire dans son origine phylogénétique comme ontogénétique du seul fait qu'il nous paraît constituer un ensemble fonctionnellement unitaire. Ainsi faut-il se défier de toute explication simple - par discontinuité comme par continuité strictement linéaire - de l'apparition d'un caractère complexe, qu'il soit organique, fonctionnel (comme la locomotion bipède) ou élément de la structure sociale (comme la monogamie).

Inattendu pour le non-spécialiste, un bon exemple de la complexité de l'évolution de caractères fonctionnellement unitaires est donné par la parturition humaine. Elle se distingue de celle des autres primates par d'importantes particularités que l'on explique par la conjonction de l'augmentation du volume du crâne du nouveau-né découlant de l'encéphalisation et de la modification du pelvis liée à la bipédie. K. Rosenberg énonce dans ses conclusions d'une analyse détaillée : "Les modalités de la naissance chez les hommes modernes paraissent avoir évolué en mosaïque, comme on pouvait s'y attendre puisque les contraintes qui ont abouti au processus actuel (efficacité de la locomotion et dimension de la tête de l'enfant) ont évolué à des moments différents. Certains aspects de la parturition moderne [...] peuvent être apparus très tôt et avoir été présents chez les australopithèques, alors que d'autres [...] sont apparus il y a moins de 1,5 million d'années et peuvent avoir permis la croissance ultérieure de la capacité crânienne ${ }^{9}$.

Bien étayée pour des caractères qui ne sont certainement pas les plus complexes, une évolution en mosaïque doit être a fortiori postulée dans le développement du langage, de la conscience ou de l'intelligence.

\section{Le maintien de ressemblances: fonds commun et parallélisme}

Si l'évolution de deux lignées sœurs indépendantes aboutit donc à des différences complexes, un autre aspect de l'évolution adaptative réside dans le fait qu'elle engendre simultanément des modifications grossièrement parallèles. À travers les brillantes œuvres de vulgarisation de S.J. Gould, l'idée de contraintes héritées du passé, limitant

\footnotetext{
${ }^{8} C f$. Dutour et al., 2005. Il s'agit de la façon la plus moderne, conforme à la théorie néodarwinienne, d'aborder le problème de l'évolution humaine.

${ }^{9} \mathrm{~K}$. Rosenberg, cité in : Dutour et al., 2005.
} 
les directions évolutives possibles des espèces, est maintenant bien connue. Le concept d' « opportunité évolutive »- qui ne se confond pas exactement avec celui, plutôt obscur d'ailleurs, de «préadaptation »- peut expliquer que deux lignées sœurs puissent se ressembler non seulement en raison du fonds commun initial qu'elles continuent à partager, mais également à travers l'acquisition plus ou moins parallèle de caractères neufs sous l'effet de la sélection naturelle. L'atteinte d'un certain état par une population ancestrale peut en effet offrir les conditions pour que de nouvelles pressions de sélection apparaissent dans la descendance et se manifestent de façon plus ou moins semblable dans ses diverses lignées. La réalisation dans une espèce souche d'un certain type d'organisation sociale conjointement à un certain mode de recherche de la nourriture a des chances d'induire des pressions de sélection assez semblables dans les différentes lignées filles en direction d'une amélioration de la communication sociale, de l'usage d'outils, des capacités d'apprentissage... Assez semblables, mais jamais identiques car la sélection naturelle n'est pas une force extérieure qui vient s'appliquer sur l'espèce, mais est elle-même le résultat de la confrontation entre la variabilité génétique des populations et les conditions de vie des individus.

Il n'y a donc pas à s'étonner du développement de ce que l'on appelle parfois des «proto-cultures » dans d'autres espèces ou sous-espèces que Homo sapiens sapiens; il n'y a pas à s'étonner non plus du caractère si souvent composite à nos yeux de fossiles d'Hominidés qui traduisent soit un degré inachevé du processus d'hominisation, soit une évolution grossièrement parallèle et sans suite dans une espèce-sœur de celle qui figurait dans notre ascendance directe, soit encore une hybridation entre lignées encore imparfaitement séparées.

\section{Conclusion}

Jusque vers le milieu du $\mathrm{Xx}^{\mathrm{e}}$ siècle, presque toute découverte d'une combinaison nouvelle de caractères chez un hominidé fossile s'accompagnait de la dénomination d'une espèce nouvelle. La raison prépondérante était certainement, plus que la gloriole attachée à la désignation d'une espèce nouvelle, la dominance d'une représentation discontinuiste de l'évolution. Elle a en effet perduré plus longtemps chez les paléontologistes que chez les autres naturalistes et n'a commencé à y être sérieusement battue en brèche qu'après la publication de Rythmes et modalités de l'évolution de G.G. Simpson en 1944. Les auteurs actuels, beaucoup plus prudents, tendent à raisonner en termes de populations, ce qui correspond au cœur de la démarche de l'écologie évolutive.

Cette dernière éclaire de nombreux aspects des processus évolutifs qui ont conduit à la distance actuelle entre l'homme et les animaux les plus proches, mais si l'on se persuade que la question des frontières entre l'homme et l'animal n'est pas celle de la frontière mythique qui, dans l'évolution, aurait présidé à l'avènement de l'humanité, mais celle des frontières actuelles dans nos représentations, alors la biologie doit rester modeste. Son rôle doit demeurer un peu semblable à celui de la fameuse échelle de Wittgenstein, qu'il invite à repousser après en avoir gravi les échelons : écartant les faux problèmes et les fausses représentations et se contentant d'affirmer que le fil du lien avec nos ancêtres n'a jamais été coupé, la biologie laisse entièrement ouvert à d'autres disciplines le champ des réflexions sur nos relations présentes avec les animaux et sur l'éthique qui devrait y présider. 



\title{
Chapitre 2
}

\section{La monogamie humaine est-elle « naturelle »?}

\author{
Frank CÉZILLY
}

Historiquement, la position radicale consistant à établir une ligne de démarcation entre animalité et humanité n'a longtemps eu pour seule préoccupation que de conserver au sujet humain un statut d'exception. Cette position demeure encore quelque peu dominante en France où la relation homme-animal est le plus souvent abordée du point de vue de l'altérité (Lestel, 1996). Ailleurs, là où l'héritage cartésien est peut-être moins pesant, une autre conception, teintée d'évolutionnisme, a pu émerger. Celle-ci recherche dans la nudité morale de l'animal le reflet d'une essence humaine étouffant sous les habits neufs de la culture (Fisher, 1992 ; Wright, 1995 ; Buss, 2003) Le discours contemporain sur la monogamie humaine témoigne parfaitement de ce clivage entre deux conceptions opposées (Cézilly, 2006). L'une célèbre la monogamie en tant que rupture fondatrice de l'espèce humaine. L'autre se désole d'une culture censée avancer à contre-courant d'un déterminisme biologique ancré dans nos gènes. Dans le premier cas, la référence à la nature vise à lui retirer tout rôle prescriptif, alors que dans le second, elle consiste à réintroduire une réalité normative en décalage avec la norme culturelle. De ce point de vue, le discours sur la monogamie humaine se confond quelque peu, et ce n'est guère surprenant, avec le discours sur la sexualité (Schouman et Simard, 2006).

C'est cette ambiguïté récurrente dans la référence à la nature qui explique le titre de cette contribution. J'entreprends en effet de répondre ici à deux questions intimement liées l'une à l'autre, mais dont les domaines de validité restent, à mon sens, distincts. La première consiste à établir si l'espèce humaine provient d'une lignée ancestrale monogame et, à ce titre, présente des caractéristiques biologiques qui, d'une façon ou d'une autre, la prédisposent à être monogame. La seconde a trait à l'existence d'une norme naturelle devant faire autorité en matière de mœurs conjugales. À l'évidence, la réponse fournie à la première question sert d'argument pour répondre à la deuxième, mais la pertinence de chacune doit s'apprécier séparément. 


\section{L'espèce humaine est-elle biologiquement monogame ?}

Répondre à cette question présuppose d'être capable d'établir un consensus préalable sur le sens accordé au terme «monogame ». Que désigne en fait la monogamie ? Pour les anthropologues et les ethnologues, le terme renvoie à une prescription sociale qui impose à chaque individu de n'être marié qu'à un seul individu. Pour le généticien des populations, la monogamie est un système de reproduction qui, sous l'hypothèse d'un nombre égal de mâles et de femelles dans une population, maximise la diversité génétique de cette population en permettant a priori à chaque individu de propager ses gènes à la génération suivante. L'écologie comportementale, quant à elle, définit la monogamie comme un régime d'appariement (Cézilly, 2006 ; Cézilly et al., 2006). Ce concept permet d'établir une typologie de l'organisation sociale de la reproduction chez les espèces animales d'après un nombre limité de critères : la durée et l'intensité des liens entre partenaires, le nombre de partenaires différents auxquels un individu est associé au cours de sa vie, le degré d'exclusivité des relations sexuelles entre partenaires reproducteurs et le mode de partage des soins parentaux entre partenaires. La monogamie se définit donc en écologie comportementale comme un régime d'appariement où un unique mâle et une unique femelle sont associés pour au moins un épisode de reproduction et manifestent une forme quelconque de coopération (défense d'un territoire commun, soins aux jeunes, comportements d'entraide ou affiliatifs (Cézilly, 2006)). Au sens strict, la monogamie implique une fidélité sexuelle réciproque entre les deux partenaires tout le temps que dure leur association.

La première question à se poser en matière de monogamie est de savoir s'il existe réellement des espèces animales se conformant à de telles exigences. La question surgit du fait que la logique de l'évolution semble a priori s'opposer à l'établissement d'un régime d'appariement monogame chez une espèce sexuée. Une telle espèce se caractérise en effet par une répartition des individus en deux groupes distincts, les mâles et les femelles. La distinction fondamentale entre les deux genres tient à leur stratégie différente de production de gamètes. Les mâles, par définition, produisent à profusion des gamètes mobiles de petite taille, les spermatozoïdes. Par contraste, les femelles fabriquent en quantités limitées de gros gamètes, garnis de substance de réserve et peu mobiles, les ovules. Cette asymétrie dans la taille et la production des gamètes est connue sous le nom d'anisogamie. Elle entraîne a priori deux stratégies de reproduction différentes pour les mâles et les femelles. Considérant que les caractéristiques phénotypiques des organismes sont l'expression de l'interaction entre leur équipement génétique et l'environnement dans lequel ils se sont développés, on acceptera qu'une information génétique n'a de chances de perdurer au fil des générations que si elle confère à l'organisme qui l'abrite une capacité à survivre et à se reproduire dans l'environnement plus importante que celles des individus abritant une information génétique différente et vivant dans le même environnement. Les mâles étant physiologiquement capables de féconder plus d'une femelle, tout gène tendant à augmenter la promiscuité sexuelle des mâles devrait être promis à un meilleur avenir qu'un gène réprimant cette promiscuité. À l'inverse, les femelles qui, chez la plupart des espèces, peuvent a priori féconder tous leurs ovules au cours d'un unique accouplement, devraient, en l'absence de bénéfice issu de la multiplication des accouplements, manifester une promiscuité sexuelle bien inférieure à celle des mâles. Cette asymétrie est à la base du processus de sélection sexuelle 
introduit par Darwin (1859 et 1871). Parce que les proportions de mâles et de femelles sont à peu près équivalentes dans les populations naturelles (on dit alors que la sex-ratio est à l'équilibre), dès lors qu'un mâle féconde plus d'une femelle, il condamne un ou plusieurs autres mâles à ne pas laisser de descendance. En résulte une concurrence entre tous les mâles potentiellement capables de féconder plus d'une femelle.

Cette concurrence a laissé des traces partout visibles dans la nature. Elle peut prendre la forme d'une compétition acerbe où chaque mâle tente par la force de s'imposer comme géniteur et développe à cet effet certains traits qui renforcent ou décuplent sa force physique, ou de manière plus subtile résulter dans une sorte de " course évolutive aux ornements » qui conduit les mâles à développer jusqu'à l'extravagance certains caractères pour lesquels les femelles manifestent un intérêt particulier. Ce phénomène, moteur de l'évolution des caractères sexuels secondaires, se manifeste par la présence ou l'amplification de certains caractères chez un seul sexe, le plus souvent les mâles (Andersson, 1994 ; Danchin et Cézilly, 2005, pp. 235-298.). Les bois des cervidés, les ergots des faisans, la queue du paon, le chant du rossignol sont autant de caractères qui ont évolué à travers le processus de sélection sexuelle, permettant aux individus de s'imposer par la force ou par la séduction. Le développement de ces caractères est particulièrement marqué chez les espèces polygynes, c'est-à-dire celles où l'unité reproductrice de base correspond à un seul mâle et plusieurs femelles. Ce qui s'explique facilement. Considérons un caractère quelconque présentant une variation interindividuelle au sein d'une population. Dès lors que cette variation est au moins en partie attribuable à des différences génétiques, la vitesse d'évolution du caractère au sein de la population sera d'autant plus importante qu'il existera une relation positive entre le succès reproducteur des individus et le degré d'expression du caractère. La polygynie, du fait de la forte asymétrie qu'elle engendre dans le succès reproducteur des mâles d'une population, catalyse donc l'évolution des caractères qui confèrent un avantage aux mâles dans l'accès aux femelles (Danchin et Cézilly, 2005, pp. 235-298.) Si le dimorphisme sexuel nous paraît aussi évident, c'est en grande partie du fait que la polygynie est, de loin, le régime d'appariement le plus fréquent chez les espèces animales. Ce simple constat est souvent invoqué comme une démonstration de la prééminence de l'anisogamie dans la dynamique qui sous-tend l'organisation sociale de la reproduction. Mais à y regarder de plus près, on réalise vite que l'anisogamie joue certes un rôle important, mais loin d'être déterminant. Le simple fait qu'à côté d'une majorité d'espèces polygynes existent des espèces monogames, et même, à une faible fréquence, des espèces polyandres (c'est-à-dire caractérisées par une unité reproductrice de base formée d'une unique femelle et de plusieurs mâles (Cézilly et al., 2006)), indique que la polygynie n'est pas une fatalité du point de vue de l'évolution des espèces. En fait, le potentiel polygyne des mâles qui découle de l'anisogamie ne peut pleinement s'exprimer que si les conditions environnementales le permettent. Dans le cas contraire, ce potentiel peut être réfréné au point que l'espèce en vient à acquérir certaines caractéristiques physiologiques et/ou comportementales qui canalisent son tempérament monogame ou polyandre. Une telle révocation évolutive de la polygynie s'est certainement produite, à plusieurs reprises et de façon indépendante, dans différents groupes zoologiques au cours de l'évolution (Cézilly, 2006).

Comment les conditions du milieu peuvent-elles contenir, voire réprimer, le potentiel polygyne des mâles? D'un point de vue évolutif, le simple fait de multiplier les accouplements ne suffit pas pour qu'un mâle maximise la diffusion de ses gènes à la génération 
suivante. Encore faut-il que la progéniture qu'il aura engendrée voit le jour, prospère et soit à même de se reproduire elle-même un jour. Cela nécessite que les femelles aient accès aux ressources de l'environnement qui conditionnent leur succès reproducteur. C'est précisément la distribution de ces ressources et leurs conditions d'exploitation qui guideront la distribution des femelles dans l'espace, et, à partir de là, celle des mâles (Cézilly et al., 2006). Si les ressources sont distribuées de manière hétérogène, certaines portions de l'habitat en concentrent la majeure partie alors qu'ailleurs ces mêmes ressources font défaut, il s'ensuivra que les femelles auront tendance à être ellesmêmes regroupées dans l'espace. Il sera alors possible pour un mâle de monopoliser une portion de ressources suffisante pour subvenir aux besoins de plusieurs femelles et de leur progéniture. Dans le cas contraire, le territoire qu'un mâle sera capable d'acquérir et de défendre ne pourra convenir qu'aux exigences d'une seule femelle. La polygynie sera alors une impasse économique. Dès que les conditions du milieu invalident la polygynie, la situation à laquelle les mâles font face est radicalement différente. Chercher à multiplier les accouplements n'est plus une stratégie gagnante d'un point de vue évolutif. S'ouvre alors la possibilité que les mâles augmentent leur succès reproducteur et la diffusion de leurs gènes en assistant leur unique femelle et en contribuant aux soins parentaux. Au sein d'une population placée dans un environnement où le potentiel de polygynie ne peut s'exprimer, tout mâle qui s'investit dans une relation de coopération avec son partenaire reproducteur a la faculté de laisser des descendants en plus grand nombre et/ou de meilleure qualité que ceux engendrés par un mâle dont l'activité reproductrice se limite strictement à l'acte copulatoire.

Il n'est donc pas scandaleux d'un point de vue «biologique » de rencontrer des espèces monogames dans la nature. Et de fait, il en existe un certain nombre, quoique réparties de façon plutôt inégale entre les différents phylums. Je ne procéderai pas ici à un inventaire exhaustif des espèces monogames (Cézilly, 2006), mais me concentrerai plutôt sur l'aspect pluriel que revêt la monogamie chez les espèces animales. La monogamie animale présente une assez large diversité d'expressions qui résulte de différences fondamentales entre espèces. Ces différences sont liées notamment à la longévité des espèces, à leur écologie, à leur mode de reproduction et au degré de développement de leur système nerveux et de leurs capacités cognitives. Ainsi, le problème de la durabilité des liens du couple ne se pose vraiment que pour des organismes dont la durée de vie est assez longue pour que leur reproduction s'étale sur plusieurs saisons, à l'instar de nombreuses espèces d'oiseaux. De même, l'importance des soins bi-parentaux ne peut se faire sentir que chez des espèces produisant des jeunes dont le développement est relativement lent. Chez certains poissons où les jeunes prennent la forme de larves planctoniques qui, dès la naissance, se dispersent en dehors du territoire parental, la logique économique de la monogamie réside tout entière dans les avantages que procure tout au long de l'année une défense commune de ce même territoire. Enfin, une dimension particulière de la monogamie animale mérite d'être soulignée. Elle concerne le découplage entre monogamie sociale et monogamie sexuelle (ou génétique). L'existence d'un lien social, qui éventuellement se prolonge au cours du temps, ne permet pas de préjuger d'une fidélité sexuelle absolue. Loin de là. Les techniques moléculaires ont permis de démontrer depuis un certain temps déjà que la fidélité sexuelle, au niveau des populations ou des espèces, est l'exception plutôt que la règle (Cézilly, 2006). Cela signifie que l'on trouve régulièrement au sein des espèces socialement monogames des mâles prodiguant des soins parentaux 
à tout ou partie d'une progéniture qu'ils n'ont pas engendrée. Sans pour autant qu'un couplage entre fidélité sociale et fidélité sexuelle soit inexistant dans la nature. Certaines espèces de corvidés ou d'oiseaux marins semblent se conformer à cette double exigence, et il est certain que chez beaucoup d'espèces socialement monogames de nombreux individus ne connaissent qu'un seul partenaire sexuel au cours de leur vie.

La diversité du vivant apparaît donc incompatible avec un modèle standard de la monogamie destiné à servir d'étalon universel. D'où une première difficulté pour juger du caractère naturel de la monogamie humaine. On peut alors se réfugier derrière l'argument de la continuité et tenter d'exhumer une lignée monogame ancestrale d'où proviendrait l'espèce humaine. Mais cette tentative est elle aussi vouée à l'échec. Ou du moins, il n'est guère possible de faire remonter l'origine animale de la monogamie humaine dans la nuit des temps. La monogamie n'est guère de mise chez les espèce de primates qui se trouvent être les plus proches de l'espèce humaine d'un point de vue phylogénétique. Ni les chimpanzés, ni les bonobos, qui sont les descendants actuels des espèces de primates dont la lignée qui a conduit à l'humain s'est irrémédiablement séparée il y a environ cinq millions d'années, ne sont monogames. Il en va de même de ces autres lointains cousins que sont les gorilles et les orangs-outans (Cézilly, 2006).

Cinq millions d'années d'évolution humaine représentent cependant un nombre de générations assez conséquent pour que des changements adaptatifs se produisent. Il reste donc possible d'imaginer que la monogamie se soit substituée à la très probable polygynie des ancêtres communs à l'homme et aux chimpanzés, après la séparation entre les deux groupes. Mais où et quand? C'est là que les avis divergent. Certains conçoivent une hypothétique adoption d'un régime d'appariement monogame par nos ancêtres hominidés comme un événement fondateur de l'espèce humaine, au même titre que la bipédie (Lovejoy, 1981, pp. 341-350). Mais ce n'est à l'évidence pas l'avis de la psychologie évolutionniste, qui considère à l'inverse que la polygynie était la règle tout au long du Pléistocène (c'est-à-dire entre environ 1,8 million d'années et 10000 ans avant notre ère), période au cours de laquelle le psychisme humain se serait modelé (Cartwight, 2000). Un critère régulièrement invoqué pour juger du degré de monogamie ou de polygynie de nos ancêtres hominidés est l'étendue du dimorphisme sexuel. D'une manière générale, on constate parmi les espèces animales une association étroite entre dimorphisme sexuel et régime d'appariement. Chez les espèces monogames où la compétition entre mâles est limitée, le dimorphisme sexuel est typiquement modéré. Cette règle est assez bien respectée par les primates, d'où l'idée que le dimorphisme sexuel mesuré chez les australopithèques puisse servir d'indice pour juger du caractère ancestral ou non de la monogamie. Malheureusement (ou heureusement), les choses ne sont pas si simples. Et ce pour deux raisons majeures. D'une part les considérations sur l'étendue du dimorphisme sexuel des hominidés sont sujettes à caution. Sur la base d'un même échantillon il est possible de conclure à l'existence d'un dimorphisme similaire à celui observé chez l'homme moderne (Reno et al., 2003, pp. 9404-9409), ou au contraire largement supérieur (Plavcan et al., 2005, pp. 313-320). D'autre part, notre reconstruction de l'évolution de l'homme n'est pas à ce point parfaite qu'il nous soit possible de connaître nos ancêtres directs parmi la diversité des espèces appartenant au genre Australopithecus. Le dimorphisme semble cependant s'atténuer au cours des quatre derniers millions d'années, mais ce n'est pas là le seul changement directionnel majeur observable au sein des lignées d'où provient l'homme moderne (Flinn et al., 2005, pp. 10-46) : il faut y ajouter un triplement 
du volume cérébral et un quasi-doublement de la période de développement. Quant au dimorphisme sexuel des humains d'aujourd'hui, il convient d'abord de noter qu'il varie entre populations (Patriquin et al., 2005, pp. 119-127) sans pour autant pouvoir le corréler à une variation dans le régime d'appariement. En comparaison des primates, les différences de stature entre hommes et femmes sont plus marquées que celles couramment observées chez les espèces de primates monogames, mais bien inférieures à celles mesurées chez les anthropoïdes polygynes.

Certaines données génétiques tendent à valider l'hypothèse d'une transition de la polygynie vers la monogamie au sein de l'espèce humaine il y a environ 5 à 10000 ans (Dupanloup et al., 2003, pp. 85-97), avec cependant un certain degré de variation entre les populations vivant sous les tropiques et celle vivant plus au nord, variation qui pourrait être liée à des différences entre populations dans le mode dominant d'exploitation des ressources (Frost, 2006, pp. 85-103). La prédominance de la polygynie jusqu'à une période relativement récente est peut-être aussi à rapprocher de la tendance des groupes humains à manifester une philopatrie mâle, c'est-à-dire une tendance plus forte des mâles que des femelles à demeurer au sein de leur groupe natal une fois atteint l'âge reproducteur (Flinn et al., 2005, pp. 10-46). Si l'existence d'un fort degré d'apparentement entre certains mâles au sein des groupes sociaux a pu jouer un rôle déterminant dans la constitution d'alliances, particulièrement efficace lors des conflits entre groupes, elle a pu aussi favoriser une polygynie de type clanique au sein des groupes. L'augmentation de la taille des groupes humains, jointe à la différenciation des tâches issue du développement technologique, aurait petit à petit imposé un fonctionnement plus égalitaire entraînant une diminution du pourcentage d'hommes polygynes au sein des populations humaines (Alexander, 1987 ; MacDonald, 1990, pp. 195-238). La complexité du phénomène est encore augmentée par le découplage entre monogamie sociale et monogamie sexuelle.

Au final, il est bien difficile de délivrer à l'espèce humaine un certificat biologique d'authenticité monogame. Cette difficulté tient pour beaucoup au fait que la monogamie n'est pas à proprement parler un trait biologique, à l'égal du nombre de dents, de la taille de portée ou du nombre de neurones dans le cerveau. La monogamie, considérée au niveau des individus, serait certainement mieux appréhendée en tant que tableau comportemental polymorphe, c'est-à-dire sous la forme d'une suite de traits dont tous ne sont éventuellement pas présents, ou du moins pas exprimés avec la même intensité, chez toutes les espèces considérées comme monogames, voire même chez tous les individus d'une même espèce. La monogamie, en tant que régime d'appariement, c'est-à-dire en tant qu'état caractéristique majoritaire d'une espèce ou d'une population, reste avant tout une solution à un problème économique, chez l'homme comme chez l'animal (Cézilly, 2006). Or, précisément, le phénomène de convergence évolutive atteste de la capacité des espèces à aboutir à un même état en empruntant des chemins différents. D'où la limite d'un raisonnement privilégiant une stricte homologie.

\section{Que vaut l'autorité morale de la nature en matière de monogamie?}

Dès le début du $\mathrm{XX}^{\mathrm{e}}$ siècle, Nicolas Berdiaeff (1935) exprimait en ces termes sa grande perplexité vis-à-vis de la monogamie humaine : 
« La monogamie ne correspond pas à la loi naturelle de l'union sexuelle. Elle n'est en aucune façon inhérente à la "nature" de l'homme, elle n'a pas toujours existé et ne s'est formée qu'à un certain stade du développement humain. Si la monogamie est possible, elle ne l'est réellement que selon la grâce, mais nullement selon la nature ou la loi. Elle est bien plus un phénomène d'ordre spirituel et mystique que d'ordre naturel et social. C'est en cela que réside son paradoxe fondamental. »

Cette insistance à dénoncer le caractère «naturel» de la monogamie démontre bien l'amalgame pratiqué de longue date entre ce qui est naturel et ce qui est bon pour l'homme, procédé largement exploité de nos jours par les publicitaires pour vanter les mérites de certains produits. On peut cependant s'interroger sur le bien-fondé d'une démarche consistant à invoquer la nature pour légitimer, ou au contraire discréditer, la monogamie humaine. Le débat sur le caractère «naturel» ou non de la monogamie me semble s'articuler autour de l'interaction entre deux critères (tableau 1). Le premier, introduit ci-dessus, concerne le caractère ancestral ou récemment apparu de la monogamie humaine. Le second a trait au problème de la rupture ou de la continuité entre l'homme et l'animal. Si bien que le problème du statut «moral » de la monogamie humaine renvoie irrémédiablement à ces deux questions.

Tableau 1. Attitudes possibles vis-à-vis de la monogamie humaine

\begin{tabular}{|c|c|c|c|}
\hline & & \multicolumn{2}{|c|}{ Relation homme-animal } \\
\hline & & Continuité & Rupture \\
\hline \multirow{2}{*}{ Monogamie } & Ancestrale & $\begin{array}{c}\text { La monogamie } \\
\text { humaine est } \\
\text { naturelle }\end{array}$ & $\begin{array}{c}\text { La monogamie } \\
\text { humaine est } \\
\text { primitive }\end{array}$ \\
\hline & Récemment apparue & $\begin{array}{l}\text { La monogamie } \\
\text { humaine est } \\
\text { mal adaptée }\end{array}$ & $\begin{array}{l}\text { La monogamie } \\
\text { humaine est } \\
\text { essentielle }\end{array}$ \\
\hline
\end{tabular}

La possibilité que la monogamie humaine représente un caractère primitif n'a reçu que peu d'attention, sans doute du fait que la monogamie est apparue peu fréquente au sein des sociétés traditionnelles. En revanche, le caractère inadapté de la monogamie, en ce sens qu'un tel régime d'appariement irait à l'encontre du déterminisme biologique du psychisme humain, est régulièrement invoqué par les tenants de la psychologie évolutionniste (Wright, 1994 ; Buss, 2003). Quant à la distinction entre un caractère « naturel »vs « essentiel » de la monogamie humaine, elle n'a au final que peu d'effet sur l'obligation morale d'être monogame qui ressort comme une déduction commune aux deux attitudes. Mais, précisément, de quelle autorité morale peuvent se réclamer ceux qui prétendent que la monogamie nous est consubstantielle ou, au contraire, radicalement étrangère ?

Pour commencer, remarquons que le concept de nature, autant que les qualificatifs qui en découlent, reste un concept imprécis. Prise au sens large, la nature désigne communément tout phénomène observable au sein de l'univers, par opposition au domaine métaphysique. On peut toutefois circonscrire la définition au monde pris dans ce qui serait sa forme essentielle, c'est-à-dire soustraite à l'influence humaine. L'adjectif « naturel» désigne alors tout ce qui est dans la nature, et, de ce point de vue, la monogamie n'est ni plus ni moins naturelle que les autres régimes d'appariement tels que la polygynie, la polyandrie ou encore la polygynandrie (Cézilly et al., 2006) qui sont tous observables, quoiqu'à des fréquences inégales, au sein des espèces animales. On débouche alors sur 
une sorte de paralogisme naturaliste mou, qui s'accommode de la diversité du vivant pour légitimer toute forme d'union matrimoniale.

Mais le concept de nature peut aussi renvoyer à un ordre logique d'essence divine, à une finalité incarnant le bien qui, en quelque sorte, obligerait à s'y conformer. De façon symétrique, la nature pourrait représenter une fatalité à laquelle il serait permis, voire souhaitable, de résister. Dans le premier cas, la monogamie serait donnée comme essence à 1'Homme par le Créateur. Dans le second, elle serait la marque de fabrique d'une identité humaine qui, précisément, se construirait par rupture avec la nature. Il est intéressant, de ce point de vue, de noter que l'épistémologie évolutionniste (von Schilcher et Tennant, 1984 ; Wuketis, 1990) a régulièrement pris soin d'expliquer que la logique de l'évolution (entendue au sens de la propagation des gènes) ne constituait aucunement une prescription morale pour l'espèce humaine. Au contraire, il pourrait même être de notre responsabilité de lutter collectivement contre le dictat du déterminisme biologique à travers l'adoption de règles sociales. Comme l'a fait remarquer le paléontologue Niles Eldredge (2004), ce dictat des gènes prend alors la forme d'un pêché originel, au point que la normativité religieuse et la normativité naturelle en viennent à entretenir un rapport de renforcement mutuel.

Une dernière possibilité est que le concept de nature soit assimilable à une forme d'innéisme. C'est à mon sens la position de la psychologie évolutionniste qui, en dépit des avertissements de l'épistémologie évolutionniste, semble percevoir cet innéisme comme irréversible. Mais en affirmant que la véritable identité de l'espèce humaine réside dans une logique d'adaptation à un environnement ancestral (situé de façon quelque peu arbitraire au Pléistocène), la psychologie évolutionniste s'engage sur un terrain glissant. Aux conceptions «modularistes » de la psychologie évolutionniste, on peut facilement opposer une conception selon laquelle la cognition sociale s'est progressivement organisée à partir de composants de base peu spécialisés (Adolphs, 2003, pp. 165-178; Flinn et al., 2005, pp. 10-46). Un autre problème qui est posé par rapport aux canons de la psychologie évolutionniste est de savoir si les stimuli sociaux sont traités au plan cognitif de la même manière que des stimuli non sociaux (nourriture, autre renforcement), avec en suspens la question du statut du renforcement sexuel vis-à-vis du renforcement affectif. Enfin, les résultats mis en avant par la psychologie évolutionniste, et censés démontrer l'inertie psychologique de l'espèce humaine à travers l'existence d'invariants transcendant les cultures (notamment en ce qui concerne les différences entre sexes), ne sont pas exempts de tout reproche, particulièrement du point de vue méthodologique (Cézilly, 2006). Tout cela concourt à donner peu de crédit à une position consistant à présenter la monogamie humaine comme «contre-nature ».

\section{Conclusion}

Parce que la question du caractère «naturel» de la monogamie humaine renvoie irrémédiablement à la notion de «nature humaine » elle rebondit sur le problème de l'émergence de l'homme moderne à un point précis dans le temps et l'espace. Si l'idée d'une émergence ponctuelle sert indéniablement les théories qui confèrent à l'espèce humaine un caractère unique, elle tourne le dos au fait que l'histoire de l'humanité est sans doute mieux représentée par un flux continu que par une série de sauts (Proctor, 2004, pp. 466-490). En même temps, l'idée d'un déterminisme biologique ancestral 
invalidant la monogamie comme mode d'organisation sociale ne repose que sur une foi aveugle dans la toute puissance d'un déterminisme pseudo-évolutionniste. L'impasse que constituent les conceptions naturalistes de la monogamie humaine invite à préférer à la notion de «nature » celle de "réalité », descriptive plutôt que prescriptive (Bonnardel, 2005, pp. 105-107). Au bout du compte, la caractéristique essentielle du psychisme humain pourrait bien tenir à la formidable plasticité de notre système nerveux, qui loin de figer nos conduites, permettrait plutôt un ajustement continu à l'environnement physique et social. De ce point de vue, l'espèce humaine ne serait pas plus disposée biologiquement à être monogame, polygyne ou polyandre mais aurait la capacité de s'accommoder de chacun de ces régimes d'appariement en fonction des contraintes économiques du moment. C'est peut-être même là une des raisons de la formidable expansion écologique de l'espèce humaine au cours de son histoire évolutive et peut-être une piste d'intérêt à explorer en matière de démarcation entre l'homme et l'animal. 



\section{Chapitre 3}

\section{Les fonctions cognitives élémentaires chez l'enfant et l'animal}

Valérie Camos, Jacques Vauclair

Le but de cette contribution est d'exposer succinctement les travaux menés sur l'animal et cherchant à mettre en évidence leurs capacités cognitives. Nous avons choisi de nous focaliser sur les primates non humains et de rapporter des recherches éclairant leurs compétences dans le traitement du monde physique et du monde social en comparaison avec ce qui est connu chez l'homme et plus particulièrement chez l'enfant.

\section{Traitement cognitif du monde physique : permanence de l'objet, rotation mentale et catégorisation}

Rien n'est plus critique pour la survie d'une espèce que sa capacité à manipuler les objets et leur déplacement dans l'espace. Par exemple, trouver de la nourriture, ses congénères ou ses petits sont des buts quotidiens de l'activité dans toutes les espèces animales (pour une synthèse, Tomasello et Call, 1997). Comprendre le déplacement des prédateurs est également une habileté absolument fondamentale. Dans cette partie consacrée au traitement cognitif du monde physique, nous décrirons trois grandes habiletés cognitives : la permanence de l'objet, la rotation mentale et la catégorisation.

\section{La permanence de l'objet}

Le concept de permanence de l'objet défini par Piaget concerne l'existence d'un objet fixe, permanent. Par la construction de cette permanence, l'enfant admet qu'un objet continue d'exister même s'il n'est plus dans son champ perceptif (Vauclair, 2004). 


\section{Stades piagétiens de la permanence de l'objet.}

Les tâches utilisées avec les primates non humains sont très similaires à celles créées par Piaget afin d'étudier la permanence de l'objet chez l'enfant. Leur principe général est de cacher des objets aux bébés et d'observer leurs comportements de recherche. Par exemple, on montre un jouet à l'enfant puis on le cache sous une couverture. La question est de savoir si l'enfant va soulever la couverture pour récupérer l'objet, ce qui prouverait qu'il a une certaine connaissance que l'objet continue d'exister même s'il ne le voit plus. Chez l'enfant humain, cette tâche est réussie à partir de 8 mois. C'est ce que Piaget a nommé le stade 4 de la permanence de l'objet. Une autre tâche classique consiste à cacher un objet sous un cache A puis après plusieurs essais réussis où l'enfant récupère l'objet sous A, l'expérimentateur déplace l'objet sous un autre cache B. L'enfant peut suivre du regard ce déplacement et il doit aller chercher l'objet sous B. L'enfant humain réussit cette épreuve à partir de 12 mois (stade 5 de la permanence de l'objet). La fameuse erreur « A-non-B » produite avant cet âge et où l'enfant persiste à chercher sous A un objet qui maintenant est caché en B a reçu plusieurs interprétations. Tout d'abord, pour Piaget, cela montre que l'enfant ne différencie pas l'objet de ses déplacements. Par contre, pour Bremmer (1980, pp. 281-309), l'enfant utiliserait un code spatial égocentrique. Enfin, pour Diamond (1991, pp. 67-110), l'enfant ne parvient pas à inhiber la recherche vers un lieu qui était préalablement porteur de réussites. Une dernière épreuve demande de « suivre » un déplacement invisible. La tâche consiste à placer un objet sous une boîte. Cette boîte est ensuite cachée sous divers contenants, et l'objet est laissé sous un des contenants. Bien que le sujet ne puisse pas savoir où l'objet a été déposé, réussir cette tâche demande de faire une recherche systématique sous chacun des contenants où la boîte sera passée. Les enfants réussissent cette épreuve à partir de 18-24 mois (stade 6 de la permanence de l'objet). Dans la théorie piagétienne, cette recherche systématique indique que l'enfant a la connaissance que les objets doivent être le long de la trajectoire et pas ailleurs (où ils ne sont pas passés). Pour Piaget, seul le stade 6 de la permanence de l'objet nécessite une représentation mentale. Dans les stades 4 et 5 , une simple image statique de l'objet suffit pour réussir les épreuves. Dans le stade 6, il faut pouvoir se représenter mentalement les déplacements de l'objet.

\section{Stades 4 et 5 chez les animaux}

De nombreuses études ont été effectuées sur des singes de l'ancien monde (singesécureuil ou capucins), des singes du nouveau monde (macaques) et des grands singes (chimpanzés et gorilles) en reprenant les épreuves des stades 4 et 5 . Elles montrent que tous sont capables de réussir les deux épreuves de permanence de l'objet. Néanmoins, il est intéressant de noter qu'il y a typiquement une période lors des tests où les animaux font des erreurs A-non-B, comme ce qui est observé chez l'enfant. Par exemple, Visalberghi (1986, pp. 445-452) montre qu'un gorille de 17 mois peut suivre les déplacements visibles d'un objet. Cependant, lorsqu'il est déplacé de $180^{\circ}$ de l'autre côté du dispositif, il commet des erreurs A-non-B. Le gorille persévère dans ses réponses en A par rapport à son propre corps, ce qui est interprété comme l'utilisation d'une référence égocentrique et non allocentrique (comme la disposition de la pièce ou la couleur de certains murs, $c f$. Bremmer, 1980). Néanmoins, ce résultat peut également être interprété comme un manque d'inhibition, comme l'a suggéré Diamond (1991), le gorille persévérant dans sa réponse. 
On notera enfin que des études similaires ont également été menées chez d'autres espèces animales. Par exemple, certaines espèces de poulets continuent à chercher la nourriture. La plupart des mammifères ont des conduites de recherches actives; les plus étudiés sont les chats et les chiens qui réussissent aussi bien les deux types d'épreuves (Doré, 1990, pp. 359-370 ; Gagnon \& Doré, 1993, pp. 246-254).

Enfin, Vauclair (1984, pp. 321-328) remarque que la réussite à ces épreuves apparaît plus tôt dans le développement des primates non humains que dans celui des humains, ce qui est probablement dû à des habiletés motrices plus tardives chez ces derniers.

\section{Stade 6 chez les animaux}

Néanmoins, pour Piaget, seule la réussite à l'épreuve de stade 6 marque véritablement la maîtrise de la permanence de l'objet. Natale, Antinucci, Spinozzi et Pòti (1986, pp. 335-339) ont testé le stade 6 chez des primates, tout d'abord avec un macaque japonais. Le dispositif est composé d'une petite boîte et de deux grandes boîtes A et B. La petite boîte sous laquelle est placée de la nourriture va être déplacée sous une grande boîte $(\mathrm{A})$ où la nourriture restera. Puis, la petite boîte est placée à côté de cette grande boîte. L'idée est donc que la nourriture est soit sous la petite boîte soit sous A, et donc la recherche doit se faire soit sous la petite soit sous A mais jamais sous B. Les réponses du macaque sont notées : « erreur » si l'animal cherche en B, "réponses séquentielles » s'il cherche dans la petite boîte puis en A, et « réponses directes » s'il cherche sous A directement. Seules les réponses directes sont considérées comme clairement de stade 6 . Sur 27 essais, le macaque a fait 8 erreurs, 16 réponses directes et seulement 3 réponses séquentielles, ce qui conduit à penser que ce macaque a atteint le stade 6 . Seulement ce résultat ne suffit pas, car il existe de nombreuses stratégies simples qui peuvent également amener à ce résultat. Par exemple, l'animal peut employer une stratégie simple qui est de chercher sous le dernier contenant où est passée la boîte ou sous la dernière boîte soulevée par l'expérimentateur. Il faut donc créer des conditions contrôles.

Natale et al. (1986, pp. 335-339) ont mis au point deux conditions contrôles. Dans la première, on donne des « faux essais », où la nourriture est sous la petite boîte et celle-ci n'est pas mise sous une grande boîte. L'expérimentateur soulève une grande boîte, mais il n'y place pas la petite. Cette condition teste l'utilisation de la stratégie simple de chercher sous la dernière boîte soulevée. Dans cette condition et pour la moitié des essais, le macaque cherche sous la grande boîte, bien que ni la nourriture ni la petite boîte ne soient jamais allées sous la grande boîte. Dans la seconde condition contrôle, la procédure est similaire à celle de l'expérience principale sauf qu'à la fin, la petite boîte n'est plus placée à côté de la grande boîte $\mathrm{A}$ où se trouve la nourriture, mais au contraire à côté de la grande boîte $\mathrm{B}$ dans laquelle il ne peut y avoir la nourriture. En résumé, il y a toujours une grande boîte vide (B) entre les deux contenants possibles (la petite boîte ou A). Cela rend la recherche plus difficile car il faut passer au-dessus de cette grande boîte vide. Ces essais sont appelés « essais non linéaires ». Dans cette seconde condition, le macaque cherche dans la boîte vide dans $70 \%$ des essais. Les résultats de ces conditions contrôles tendent à montrer que le macaque n'a pas réellement atteint le stade 6 de la permanence de l'objet, contrairement à ce que les résultats de l'expérience principale laissaient suggérer. Dans des études similaires, avec 3 macaques à longue-queue et 2 capucins, Natale et Antinucci (1989, pp. 97-112) retrouvent ces résultats négatifs dans les conditions contrôles. En conclusion, les singes ont des stratégies pratiques pour réussir ce type de tâche, mais ils n'utilisent pas de recherches systématiques basées sur la représentation mentale de déplacements invisibles. 
Les mêmes auteurs, Natale et al. (1986), ont fait la même série d'expériences avec une jeune gorille femelle. Il semble, au contraire des singes, qu'elle ait une stratégie de recherche basée sur une représentation mentale. Dans l'expérience principale, la gorille utilise la stratégie directe. Dans la condition contrôle « faux essais », sur 12 essais, elle n'a jamais cherché sous la grande boîte qui avait été soulevée mais où rien n'avait été déposé. Dans la condition contrôle "essais non linéaires », elle passe correctement au-dessus de la grande boîte vide et cherche correctement (au-delà du seuil du hasard ; néanmoins elle commet $38 \%$ d'erreurs). Pour conclure, et contrairement aux singes, la jeune gorille semble donc bien avoir une représentation mentale des déplacements invisibles caractéristique du stade 6 de la permanence de l'objet. Cependant, c'est la seule étude avec un grand singe. Enfin, on notera que d'autres animaux semblent avoir également cette compétence. Bien que les chats ne puissent pas résoudre ce type de tâche (Doré, 1990 pp. 359-370), les chiens (surtout des terriers) utilisent la stratégie basée sur la représentation mentale (Gagnon et Doré, 1993, pp. 246-254).

Pour conclure sur la permanence de l'objet, les singes peuvent atteindre le stade 5 de la permanence de l'objet, ainsi que les chats et les chiens. Par contre, seuls les grands singes et les chiens réussissent les épreuves de stade 6. Une étude récente de Collier-Baker et Suddendorf (2006, pp. 89-97) montre que les chimpanzés manifestent des capacités similaires à celles des enfants de 2 ans dans la recherche d'un objet à la suite d'un double déplacement invisible.

\section{La rotation mentale}

La rotation mentale est une autre compétence du traitement du monde physique qui, chez l'homme, a été tout d'abord mise en évidence par Shepard et Metzler (1971, pp. 701-703). Dans leur étude qui est désormais un classique en psychologie cognitive, les auteurs présentaient deux figures géométriques complexes et demandaient aux sujets de dire si ces deux figures représentaient le même objet sous des angles différents. Le résultat majeur est que le temps mis par les sujets pour répondre dépend de façon linéaire de l'angle de rotation qui sépare les deux figures. Cette étude a permis de mettre en évidence, chez l'homme, ce processus de rotation mentale qui est similaire à une rotation dans le monde physique.

Vauclair, Fagot et Hopkins (1993, pp. 99-113) ont réitéré cette expérience avec 6 jeunes babouins (élevés en milieu naturel). Ils utilisent une procédure dite d'appariement à un modèle. Tout d'abord, un modèle est présenté sur un écran d'ordinateur au babouin (Figure 1a). Après un délai, ce modèle est remplacé par deux stimuli, l'un étant le modèle mais dans une orientation différente (tourné de $60^{\circ}, 120^{\circ}, 180^{\circ}, 240^{\circ}$ ou $300^{\circ}$ ) et l'autre étant l'image du modèle dans un miroir (Figure 1b). La tâche des babouins est de choisir parmi ces deux stimuli lequel représente le modèle. Quatre des 6 babouins ont été capables de choisir (c'est-à-dire, au-dessus du seuil du hasard) le stimulus qui s'apparie au modèle. De plus, tout comme pour les humains de l'expérience de Shepard et Metzler (1971), les temps de réponse dépendaient de l'angle de rotation du stimulus. Ainsi, les babouins ont un processus de rotation mentale similaire à celui de l'homme. On notera qu'un tel processus n'est pas présent dans toutes les espèces animales. Ainsi, bien que les pigeons puissent discriminer les stimuli et ceci quelle que soit leur orientation, leur temps de réponse ne dépend pas de l'angle de rotation des objets (Hollard et Delius, 1982, pp. 804-806). Une autre espèce, l'otarie semble également capable de 
rotation mentale partielle. Ainsi, Mauck et Dehnhardt (1997, pp. 1309-1316) rapportent chez ce mammifère marin (une seule otarie testée) une augmentation linéaire du temps de réaction dans la discrimination de formes visuelles asymétriques entre $0^{\circ}$ et $120^{\circ}$, puis une diminution du temps pour $150^{\circ}$ et $180^{\circ}$.

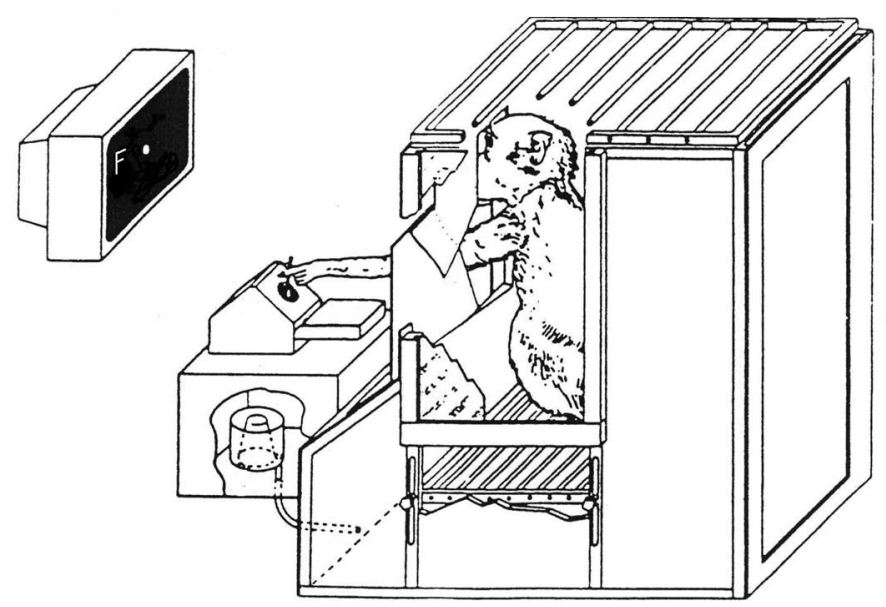

Figure 1a. Dispositif expérimental pour tester la rotation mentale chez le singe (babouin) (d'après Vauclair, Fagot \& Hopkins, 1993).

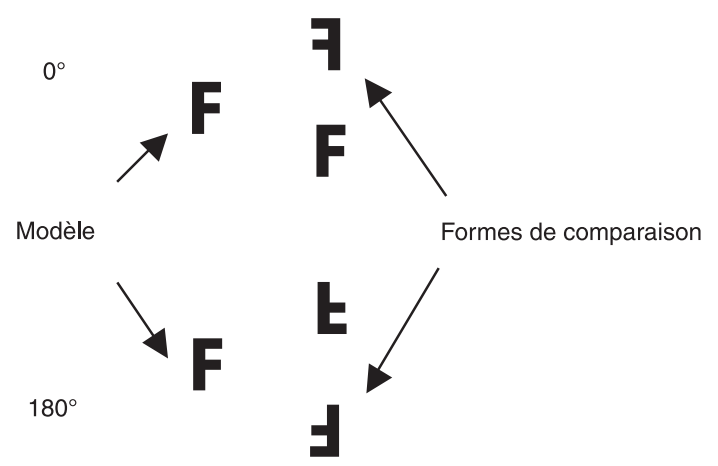

Figure 1b. Deux conditions de présentation des stimuli visuels $\left(0^{\circ}\right.$ et $\left.180^{\circ}\right)$ dans l'expérience (d'après Vauclair, Fagot \& Hopkins, 1993).

\section{La catégorisation}

La catégorisation est l'habileté à constituer des classes d'objets. Les enfants commencent à faire des classifications de façon spontanée dès 1-2 ans (par exemple, les poupées d'un côté et les nounours de l'autre), et ils trient les objets selon des critères similaires à ceux de l'adulte à partir de 3-4 ans (Langer, 1986). Dans leur environnement naturel, les singes montrent également des comportements qui indiquent une classification naturelle des objets. Par exemple, ils vont chercher certains types d'aliments ou 
n'utiliser que certains types de matériaux végétaux pour faire leur nid. Une façon de tester expérimentalement cette habileté est de présenter simultanément différents objets et de « demander » aux animaux de les trier sur la base de leurs ressemblances et différences. Ainsi, Weinstein (1945, pp. 2-48) a entraîné un macaque rhésus à trier des objets sur leur couleur. Par exemple, le singe devait enlever du plateau qui lui était présenté les objets rouges lorsque le modèle était rouge. Bien que le macaque réussisse cette tâche, ce tri est limité puisqu'il ne conduit qu'à un seul groupe (les rouges).

Garcha et Ettlinger (1979, pp. 213-224) ont mené une étude dans laquelle 5 chimpanzés, 6 macaques rhésus et 3 capucins devaient effectuer des tris en 3 groupes. Face à 3 récipients transparents, 9 objets en bois (différant en forme, taille et couleur) devaient être triés en 3 groupes. Pour le premier objet, l'animal avait toujours une récompense car a priori le tri est correct. Ce n'est que pour le deuxième que l'on peut juger du critère choisi par l'animal pour le tri. Aucun singe (capucin ou macaque) n'a été capable de faire le tri (c'est-à-dire, au-dessous du seuil du hasard) sur les 100 premiers essais. Un macaque et 2 capucins ont réussi à atteindre le seuil du hasard au bout de 880 à 2418 essais. Par contre, 4 chimpanzés (sur 5) ont réussi au-delà du seuil du hasard à faire le tri en 3 groupes ( 2 lors des 100 premiers essais et 2 lors des 650 essais). Dans un test suivant, 2 de ces chimpanzés ont même pu trier un nouvel ensemble d'objets selon des critères de taille ou de couleur (la forme semble beaucoup plus difficile à appréhender).

Avec une tâche plus complexe, Braggio, Hall, Buchanan et Nadler (1979, pp. 10971097) ont également testé des chimpanzés. Six jeunes chimpanzés devaient effectuer une tâche de classification multiple sur une matrice $2 \times 2$ où une case était à compléter (Figure 2). Cela demande donc la manipulation conjointe de deux critères de classement (dans l'exemple présenté, la taille et la forme). Très rapidement, les chimpanzés ont réussi à compléter cette matrice. Cependant, comme le notent Doré et Dumas (1987, pp. 219-233), il est possible que les animaux aient utilisé une stratégie plus simple car l'objet à choisir (le petit carré) était toujours présenté avec les mêmes formes que celles présentes dans la matrice. Par simple comparaison avec les formes présentes dans la matrice, les chimpanzés peuvent donc déterminer l'objet à choisir.

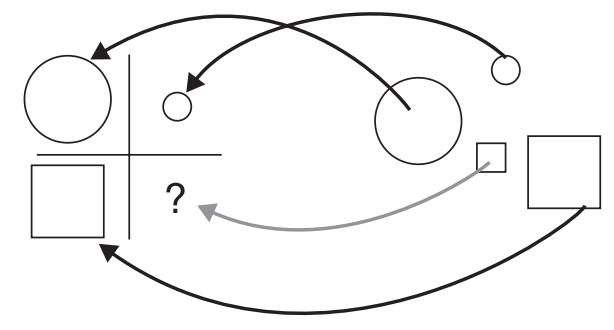

Figure 2. Matrice $2 \times 2$ et objets utilisés dans l'expérience de Braggio et al. (1979, pp. 1097-1097).

Les singes comme les capucins ont donc beaucoup de difficultés à effectuer des tris. Ce sont principalement les grands singes, quand ils ont été élevés par des humains, qui montrent les meilleures capacités de catégorisation. On notera enfin que les chimpanzés, qui sont de naturels utilisateurs d'outils, sont les seuls grands singes qui ont été observés en train de faire de telles classifications hors de tout entraînement ou influence humaine. 


\section{Traitement cognitif du monde social : représentation de soi et attribution de savoirs}

Parallèlement aux travaux menés sur la représentation et le traitement des objets, des recherches sont conduites sur la représentation de soi, et sur les capacités à attribuer des savoirs aux autres individus.

\section{La représentation de soi : la reconnaissance de soi dans le miroir}

Dans la recherche sur les questions de perception de soi, reconnaissance de soi et de concept même de soi, la méthodologie habituellement utilisée est l'étude de la réaction des individus face à leur image dans un miroir.

\section{Chez l'enfant}

Chez l'enfant humain, on décrit trois grandes phases dans les comportements face à son image dans le miroir (Fontaine, 1992; Mounoud et Vinter, 1981; Zazzo, 1975, pp. 389-413). De 6 à 15 mois, l'enfant placé devant un miroir va sourire à son image, il vocalise. Il cherche derrière le miroir cet autre enfant qu'il a en face de lui. À partir de 15-18 mois, apparaissent des comportements de rupture. L'enfant va regarder de biais cette image dont les mouvements sont si synchrones avec les siens. Il est embarrassé et préfère même s'en détourner. Ce n'est qu'à partir de 24 mois que l'enfant se reconnaît dans cette image, nommant son reflet par son prénom ou « moi ». Il va même commencer à utiliser le miroir. Ainsi, si on place une tache d'encre rouge sur le front de l'enfant, il pourra grâce au miroir toucher et frotter cette tache. De tels comportements ne sont possibles que si l'enfant a acquis la reconnaissance de soi.

\section{Chez l'animal}

Gallup (1970, pp. 86-87) a étudié les réactions face au miroir de 4 chimpanzés et de 6 macaques. Chaque animal a été exposé face au miroir 8 heures par jour pendant 10-14 jours. Gallup a noté une différence marquée dans le comportement des chimpanzés pendant cette période. Dès le $3^{\mathrm{e}}$ jour, il a observé un accroissement marqué des comportements dirigés vers soi et nécessitant le miroir, par exemple, épouiller des parties du corps non visibles ou enlever des morceaux de nourriture entre les dents. Ces comportements sont très différents de ceux que les chimpanzés peuvent avoir face à des congénères. Par contre, pour les macaques, très peu de comportements dirigés vers soi ont été observés sur l'ensemble de la période d'étude.

Dans une seconde phase, les sujets ont été marqués avec une tache rouge (au-dessus d'un sourcil) qui n'était pas visible pour le sujet. Ces taches ne laissent ni indices tactiles ni odeurs. Les individus sont ensuite placés devant un miroir. Les chimpanzés se comportent de telle sorte qu'ils comprennent que ces taches sont sur leur propre corps. Par exemple, ils touchent cette tache plus que toutes les autres parties de leur corps. Ils inspectent leurs doigts après avoir l'avoir touché et parfois ils reniflent leurs doigts. Des études similaires ont été faites avec d'autres grands singes. Les orangs-outans et les bonobos se comportent comme les chimpanzés face au miroir. Par contre, les résultats avec les gorilles sont plus mitigés. Suarez et Gallup (1981, pp. 239-244) ne répliquent pas les comportements dirigés vers soi dans l'épreuve de la tache rouge. Cependant, Patterson montre que son gorille Koko se reconnaît dans le miroir et réussit l'épreuve de la tache rouge (Patterson et Cohn, 1994, pp. 273-290). 
À l'inverse, les singes comme les macaques ne manifestent aucun de ces comportements. De nombreuses études menées sur les singes ont montré qu'ils n'avaient pas de comportements de reconnaissance dans le miroir. Cependant, il est possible que la tache ne soit pas suffisamment perceptible pour eux. Hauser et al. (1995, pp. 10811-10814) ont montré que les tamarins pouvaient réussir l'épreuve de la tache si celle-ci était très visible, c'est-à-dire, placée sur le devant de la tête dans le pelage blanc. Il est également possible que les singes ne sachent pas se servir du miroir pour repérer la tache. Enfin, des analyses détaillées des réactions face à l'image spéculaire indiquent que les singes capucins réagissent différemment à leur image et à celle d'un congénère (plus de contacts visuels et moins de réactions de peur devant l'image spéculaire). Il est probable que ces singes montrent ainsi un niveau intermédiaire entre percevoir leur propre image et percevoir l'image d'autrui (De Waal et al., 2005, pp. 1140-1147).

Des recherches plus récentes ont également mis en évidence des différences développementales et individuelles. Swartz et Evans (1991, pp. 189-206) rapportent qu'1 sur 11 de leurs chimpanzés a pu réussir l'épreuve de la tache rouge. Povinelli et al. (1994, pp. 74-80) montrent que, même après plusieurs heures d'exposition, seulement $26 \%$ de leurs 35 chimpanzés adultes (âgés de plus de 16 ans) se reconnaissaient dans le miroir (c'est-à-dire, avaient au moins 5 comportements d'exploration dirigés vers des parties du corps non visibles sans le miroir), $75 \%$ des 12 adolescents (âgés de 8 à 15 ans), $26 \%$ de leurs 10 enfants (6-7 ans) et 1 sur 48 des très jeunes chimpanzés (de 1 à 5 ans). À l'exception d'un chimpanzé, aucun ne pouvait réussir l'épreuve avant l'âge de 4 ans et demi. Lin et al. (1992, pp. 120-127), en utilisant des critères plus faibles, ont montré qu'aucun de leurs chimpanzés ne pouvait réussir avant 2 ans et demi. En effet, seuls les comportements d'exploration corporelle sont de bons indicateurs de la reconnaissance de soi dans le miroir ( $c f$. étude d'Eddy et al., 1996, pp. 38-44, où les auteurs comparent les comportements face au miroir vs. les comportements face à une image vidéo d'un congénère).

\section{Comprendre les connaissances et les croyances d'autrui}

Une autre façon de tester les représentations des primates d'eux-mêmes et des autres est l'étude de ce qu'on nomme les théories de l'esprit.

\section{Chez l'enfant}

Les théories de l'esprit sont les capacités à prédire et à expliquer un comportement par des états mentaux inobservables (Premack et Woodruff, 1978, pp. 615-636). Ce sont des conceptualisations naïves que l'enfant ou l'adulte construit à propos de phénomènes psychologiques qui se produisent chez lui-même et chez autrui. Très tôt, les enfants disposent de connaissances et de représentations relatives aux entités mentales. Dès 2-3 ans, apparaît l'ébauche d'une connaissance de la perception d'autrui et les enfants commencent à utiliser des verbes de croyances et de désirs (penser, savoir, vouloir). L'évolution la plus importante se déroule entre 2 et 6 ans et elle concerne trois principaux domaines. Tout d'abord, avant l'âge de 5 ans, un enfant ne peut concevoir que deux personnes peuvent avoir des représentations différentes d'une même situation. Il a également des difficultés à distinguer la différence entre l'apparence et la réalité (par exemple, un objet peut ressembler à un livre mais être en fait une boîte (Flavell et al., 1983, pp. 95-120)). Enfin, avant 5 ans, un enfant ne peut concevoir qu'autrui puisse avoir une croyance (une représentation) qui diffère de l'état de la réalité ou de ce qu'il connaît ou croit lui-même. Par exemple, si 
on cache un bonbon dans une boîte sous ses yeux, il croira qu'un autre enfant qui n'aurait pas assisté à la scène sait également qu'il y a un bonbon dans la boîte. Ce n'est qu'à partir de 5 ans que les enfants peuvent prédire les actions d'autrui à partir des croyances (Perner et al., 1987, pp. 125-137, J. Perner \& Wimmer, 1985, pp. 437-471).

\section{Distinguer ses connaissances de celles d'autrui}

Chez les primates non humains, de nombreuses études ont cherché à évaluer ces théories de l'esprit. Tomasello et Call (1997) rapportent une étude de Premack sur 4 jeunes chimpanzés. Au cours de celle-ci, un expérimentateur place de la nourriture dans un des deux récipients situés face à un chimpanzé. Cependant, le chimpanzé ne peut voir dans quel récipient la nourriture a été déposée. Cette scène se déroule également en présence de deux soigneurs humains dont l'un seulement peut voir où a été placé la nourriture, l'autre ayant son champ visuel entravé par un panneau de bois. Ensuite, les deux soigneurs indiquent un récipient au chimpanzé et celui-ci prend un des deux récipients. Deux des quatre chimpanzés choisissent de suivre l'indication du soigneur qui a été capable de voir, et ceci dès le premier essai, ce qui indique que les chimpanzés ont compris que cet individu était en possession de connaissances que ni eux ni l'autre observateur n'avait (c'est-à-dire, où était la nourriture).

Le problème avec cette interprétation est que lorsque les chimpanzés ont plus tard participé à une expérience similaire de choix entre les deux soigneurs, mais cette fois, les chimpanzés avaient vu où était la nourriture, ils ont continué de choisir le soigneur ayant été témoin alors que ce n'était plus la peine. Ils n'avaient plus besoin de son conseil puisqu'ils leur suffisaient de choisir eux-mêmes. Cette condition soulève la possibilité que les premiers choix effectués par les chimpanzés étaient basés sur une préférence pour le soigneur témoin. On notera également que Premack donne peu de détails sur la procédure employée dans ces études.

Povinelli, Nelson, et Boysen (1990, pp. 203-210) ont donc mené une expérience similaire avec 4 chimpanzés. Tous avaient une bonne expérience des contacts humains. Ils étaient témoins d'une scène où un expérimentateur cachait de la nourriture sous une tasse parmi 4. Ces tasses étaient toutes placées sous un cache pendant la durée de l'expérience. Ainsi, les chimpanzés ne savaient pas où la nourriture avait été placée. Un autre humain était resté hors de la pièce pendant que la nourriture était cachée et ne savait donc pas où elle se trouvait. Après l'entrée de cet humain «naïf» dans la pièce, les chimpanzés pouvaient choisir entre « le naïf» et un autre humain qui, lui, était dans la pièce lorsque la nourriture était cachée et qui allait les informer. S'ils choisissaient «l'informé », ils obtenaient toujours la nourriture et s'ils choisissaient « le naïf », ils n'obtenaient jamais la nourriture. Les résultats indiquent que 3 des 4 chimpanzés choisissaient significativement « l'informé » plutôt que le « naïf», pour 2 chimpanzés en 100-150 essais et pour le $3^{\mathrm{e}}$ en 250-300 essais (taux de bon choix environ $70 \%$ sur les derniers essais).

Une seconde phase a permis de tester le transfert sur 30 nouveaux essais où un expérimentateur neutre plaçait à nouveau la nourriture sous des tasses. La différence est que cette fois le « naïf » restait dans la pièce mais avec un seau sur la tête ce qui l'empêchait de voir. "L'informé » restait lui dans la pièce comme dans l'épreuve précédente. Les 3 chimpanzés réussissaient la tâche de la même façon. Bien que ces chimpanzés n'eussent pas les habiletés sociocognitives nécessaires au début de l'expérience, puisqu'il a fallu de nombreux essais pour qu'ils réussissent, cette épreuve de transfert montre qu'ils ont 
compris que c'était bien plus que la présence physique de l'expérimentateur mais quelque chose de plus général.

Cependant, deux problèmes ont été relevés dans cette étude. Premièrement, Povinelli en réanalysant les essais de transfert a noté que pour les 5 premiers essais la réponse des chimpanzés était au hasard, et ce n'est qu'après qu'ils choisissaient l'«informé ». Ils n'avaient donc peut-être pas compris que la seule présence n'était pas utile, et s'ils répondent correctement au bout de 5 essais dans la phase transfert c'est que l'apprentissage dans la discrimination de la bonne réponse se faisait plus vite dans cette seconde phase. Le deuxième problème est que Povinelli, Rulf et Bierschwale (1994, pp. 74-80) ont essayé de réitérer cette étude avec 6 chimpanzés de 4 ans élevés en captivité et qu'aucun des chimpanzés n'a pu faire la différence entre « l'informé » et « le naïf ». Les auteurs suggèrent que c'est parce que les singes étaient très jeunes. Par ailleurs, Povinelli, Parks, et Novak (1991, pp. 318-325) ont essayé de refaire l'étude de Povinelli, Nelson, et Boysen (1990, pp. 203-210) mais avec 4 macaques. Après des centaines d'essais, aucun des macaques n'a pu significativement choisir le bon expérimentateur.

Ces études portent toutes sur la capacité des primates non humains à distinguer leurs croyances de celles d'humains. Cheney et Seyfarth (1990) ont mené une étude où les primates doivent évaluer l'état de connaissances de leurs congénères. Les auteurs ont testé des macaques japonais et des macaques rhésus, et ils ont cherché à savoir si des mères macaques vont informer leurs petits de la présence de nourriture ou d'un (pseudo) prédateur. La procédure expérimentale comprend deux conditions. Dans une condition, la mère et le petit sont assis dans une cage et regardent, soit l'expérimentateur cacher de la nourriture dans la cage voisine, soit un prédateur (un expérimentateur avec un masque) entrer dans la cage voisine et se cacher derrière une barrière proche. Dans l'autre condition, le petit est absent quand ces événements se déroulent, et seule la mère en est témoin. Dans les deux conditions, le petit est placé dans la cage voisine et la question est de savoir si la mère va se comporter différemment dans les deux conditions afin d'avertir son petit, soit de l'emplacement de la nourriture, soit du danger que représente le prédateur. Les auteurs ont montré que la mère se comportait de la même façon dans les deux conditions, et ceci bien qu'on sache que les petits se comportent eux différemment quand ils sont informés qu'il y a de la nourriture (ils la cherchent) ou qu'il y a un prédateur (ils restent proches de leur mère). Les auteurs en concluent que les macaques ne comprennent pas l'état de connaissances de leurs congénères.

L'ensemble de ces résultats montre que les capacités à distinguer ses connaissances de celles d'autrui sont plus limitées chez des primates non humains adultes que celles dont font preuve les enfants de plus de 5 ans.

\section{Conclusion}

Les résultats des différentes expériences résumées ici indiquent clairement que les primates non humains manifestent la plupart des traitements cognitifs présents chez le jeune enfant. Il est important de noter que les capacités liées à la permanence de l'objet ou à la catégorisation s'observent dans de nombreux groupes taxonomiques et ne sont donc pas l'apanage des seuls primates (Kreutzer \& Vauclair, 2004, pp. 1-20 ; Vauclair, 1995). Pour ce qui est de la compréhension des connaissances et des croyances d'autrui, y compris la reconnaissance de soi, les données actuellement disponibles sont plus 
controversées (Povinelli, Bering \& Giambrone, 2000, pp. 509-541). Un problème majeur qui n'a pas encore été résolu à ce jour est de pouvoir déterminer si les animaux, dans ces situations, raisonnent à partir d'observables (apprentissage de régularités, hypothèse non mentaliste) ou bien s'ils sont capables de raisonner à partir d'états mentaux (Heyes, 1998, pp. 101-148). Une telle capacité de raisonner à partir d'états mentaux (qui indiquerait la présence d'une forme de méta-représentation) apparaît chez l'enfant dès l'âge de 4-5 ans. En traitant, d'une part, les observables et en attribuant, d'autre part, des états mentaux, l'homme dispose d'un système à deux niveaux. La théorie de l'esprit a comme fonction de l'aider à comprendre de façon nouvelle les anciens comportements. Pour les psychologues du développement (Karmiloff-Smith, 1992), la théorie de l'esprit permettrait, grâce au langage, de « redécrire » la théorie initiale de l'action. Il reste à vérifier si de telles compétences sont possibles, en l'absence de langage, chez l'animal. 



\section{Chapitre 4}

\section{Brève histoire des idées et représentations des monstres hybrides entre l'homme et l'animal}

PierRe Ancet

La monstruosité physique réelle confronte l'observateur à de bien étranges figures : un enfant cyclope, un homme velu comme un lion, un enfant sirène aux jambes jointes en queue de poisson, un anencéphale au visage sans front, surplombé par deux yeux globuleux de batracien... Il ne s'agira pas ici d'affirmer qu'il existe des monstres en soi, de dire ce qu'ils sont en eux-mêmes, mais ce qu'ils sont pour nous, notamment à travers les différentes représentations d'êtres hybrides, mi-hommes mi-animaux, qui viennent spontanément à l'esprit. La difficulté que nous, hommes, avons à tolérer une variation de la forme humaine nous conduit bien souvent à interpréter les déformations corporelles en termes de métamorphoses de l'homme en animal. Et cela d'autant plus que les hybrides sont des figures que l'on retrouve dans toutes les mythologies populaires : loin d'être une nouveauté, la forme hybride est rapidement reconnue et rapidement projetée sur le corps difforme.

Il semble fréquent (mais non constant) dans les groupes humains d'associer la difformité à une forme d'animalité, que l'on tienne cette proximité pour une simple analogie ou pour une réalité. En effet, l'humain, pour se définir, tâche de se dissocier de l'animalité (à l'exception des cultures où l'on se définit comme humain au travers du lien à un animaltotem). De ce fait, il n'existe que peu ou pas de catégories et stéréotypes intermédiaires entre l'humain et l'animal. Il faut que la séparation entre l'un et l'autre soit nette pour se définir en tant qu'humain. Dès lors, ce qui n'est pas homme, ce qui n'en n'a plus l'apparence, se teinte rapidement d'animalité. Même un enfant né du ventre d'une femme pourra ainsi paraître échapper à son espèce d'origine et laisser se développer des fantasmes liés à un acte reproducteur fautif et bestial. Les impressions d'hybridation (de mélange entre deux espèces), de naissance transpécifique (où un être naît d'une mère d'une autre espèce) viennent probablement de cet attachement à la forme humaine et de la volonté constante de dissocier l'humain de l'animal, tout en sentant implicitement une proximité de nature entre l'un et l'autre. Mais pour ne pas nous bercer de généralités, nous nous appuierons 
sur quelques exemples historiques et anthropologiques de regards portés sur ces êtres jugés hybrides ou transpécifiques. Ces exemples auront pour fonction de nous faire réfléchir à la manière dont un regard se constitue par l'intermédiaire de connaissances préalables. Ainsi, même si les monstres n'ont jamais laissé indifférent un groupe humain, le regard varie très profondément d'une époque à l'autre, d'une culture à l'autre : il est fonction d'une conception de l'humain et d'une idée des lois de la nature. La notion même de monstre possède une extension variable, même si elle désigne toujours un écart par rapport aux régularités naturelles. Si l'animalisation du monstre humain (ou l'humanisation du monstre animal) se rencontre très fréquemment, elle ne peut avoir la même signification ni la même fonction, en raison du réseau de sens dans laquelle elle prend racine. Quelle méthode employer pour appréhender le plus universellement possible le regard sur le monstre hybride ? Pouvonsnous arriver à nous délivrer de nos propres inquiétudes pour interroger les regards du passé ? Sommes-nous certains d'être délivrés de ces représentations de monstres hybrides depuis qu'existe la science des monstres ou « tératologie » apparue au début du XIX ${ }^{\mathrm{e}}$ siècle en France avec Étienne et Isidore Geoffroy Saint-Hilaire ${ }^{1}$ ?

Nous commencerons par interroger le regard porté sur le monstre hybride à la fin du Moyen Âge et à la Renaissance, dont l'étrangeté sera un bon moyen de lutter contre notre tendance à surestimer l'universalité de nos perceptions et réactions face à la monstruosité.

\section{L'enfant à tête de chien et le veau né d'une femme}

Le monstre n'a pas toujours produit les réactions de crainte qui nous semblent aller de soi parce qu'elles sont viscérales. Que la vision de la monstruosité retentisse fortement sur l'ensemble de nos contemporains n'est pas une preuve qu'il en ait toujours été ainsi : le caractère irrépressible d'une réaction n'est pas nécessairement la marque de sa validité atemporelle et transculturelle.

Mais c'est trop peu dire encore que l'on réagit différemment selon les époques : les perceptions elles-mêmes diffèrent. La perception n'est pas un acte autonome, indépendant de la culture qui viendrait y surajouter une interprétation. Lorsqu'un homme de la fin du Moyen Âge découvre le prodige qu'est l'enfant à tête de chien ${ }^{2}$, il voit véritablement cette chimère composite, et ne se demande pas s'il est en train de s'illusionner. $\mathrm{Au}$ nom de quoi se le demanderait-il ? Comment pourrait-il avoir l'idée que ce qu'il voit doit nécessairement être un enfant difforme parce que né d'une femme ? Il ne possède pas l'idée d'une loi de la nature qui régirait la génération. Comme l'écrit F. Jacob, « Il n'existe pas encore de lois de la nature, pas plus pour régir la génération des animaux que le mouvement des astres. On ne distingue pas entre la nécessité des phénomènes et la contingence des événements » (Jacob, 1992, p. 27). Cette vision de l'enfant à tête de chien ne met pas en cause l'idée que le spectateur du Moyen Âge a de l'homme. Il ne cherche pas à le ramener à une humanité mal formée, il ne se demande pas si ce qu'il voit est de l'ordre d'une ressemblance plus supposée que constatée. Car ce qu'il voit a valeur

\footnotetext{
${ }^{1}$ L'ouvrage de référence présentant une classification ordonnée des monstres toujours utilisée aujourd'hui dans des thèses de médecine est l'Histoire générale et particulière des anomalies de l'organisation chez l'Homme et les animaux ou Traité de tératologie, 1832-1836 (3 vol.) d'Isidore Geoffroy Saint-Hilaire.

${ }^{2}$ Céard, 1996, p. 75 : «En l'an du Christ 1414, “fut présenté ung monstre qui avoit la teste come ung chien et tous les aultres membres come ung home..."».
} 
de signe. Et sa préoccupation a pour objet la lecture de ce monstre. Le monstre hybride a valeur de texte ou d'image à clé. C'est pourquoi son apparence n'est pas examinée pour elle-même : elle est perçue à travers cette valeur d'expression qui la dépasse. Même si l'homme qui le découvre ne se sent pas apte à décrypter seul le message, ce statut demeure, comme demeure aujourd'hui l'idée d'une malformation explicable même pour l'observateur moyen qui serait bien en peine de l'expliquer. Dans les deux cas, il s'agit de faire rentrer le monstrueux dans l'ordre d'un sens préétabli. Il n'est pas de perception en soi ni d'apparence en soi qui serait isolable de ce contexte culturel de découverte. Encore moins existe-t-il une réaction invariable face à l'apparition de ce que nous appelons un monstre et que la Renaissance nommait un prodige.

Pour l'homme de la fin du Moyen Âge et l'homme de la Renaissance l'être composite est plus une source d'expression qu'un objet d'examen et d'interrogation. Et la ressemblance qui s'y lit a une valeur bien supérieure à ce que nous appellerions l'analogie visuelle. Que cette tête ressemble à celle d'un chien au sens où nous l'entendons n'est d'aucun intérêt. La ressemblance n'a rien de ludique ou d'illusoire. Au contraire, elle indique une similitude plus profonde. Elle est une clé d'intelligibilité du monde. La ressemblance de forme est la marque d'une proximité au sein d'une nature où les formes renvoient les unes aux autres. Pour l'homme de la Renaissance, une ressemblance n'est pas une analogie oiseuse ou un jeu du regard humain avec les formes. Elle a valeur de connaissance. Dans la pensée de la Renaissance, écrit encore F. Jacob, «Les ressemblances ne sont ni inutiles ni gratuites. Elles ne traduisent pas un simple divertissement du ciel. C'est parce que certains corps possèdent les mêmes propriétés qu'ils se ressemblent. Inversement, la similitude traduit une communauté de qualité » (Jacob, 1992, p. 29).

Lorsqu'au début du XVII ${ }^{\mathrm{e}}$ siècle à Genève, une femme donne naissance à un veau, les membres du grand conseil de la ville ne se préoccupent pas de savoir si ce « veau» possède une forme vaguement humaine : toute la discussion porte sur l'origine « divine » ou « naturelle » de la naissance, sur la faute maternelle qui doit en être la cause, et sur le sort à lui réserver, comme en témoignent les passages suivants :

« La cloche sonne, ils s'assemblent en la salle du grand Conseil, où fust porté ledict veau [...] un quidan Philosophe d'entre eux, voulut attribuer cela aux imaginations de la mère, disant cela estre naturellement : Mais il fut très bien repoussé par un des assistans, lequel disoit que ce ne pouvoit arriver naturellement. ${ }^{3}{ }^{\prime}$

Pourtant l'impiété de la mère est retenue comme cause possible de la naissance monstrueuse, cette femme étant supposée avoir déclaré :

«J'aimerais mieux plus tost mourir, ou vrayment enfanter un veau que de permettre que l'oraison de saincte Marguerite fust dicte en mon intention: Response fort indigne, et dont [...] elle en recent tost son guerdon : Car d'un corps formé d'une ame raisonnable qu'elle avoit dans son ventre, elle sent un corps brutal, et à l'instant délivre d'iceluy, sçavoir d'un veau, ainsi qu'elle l'avait souhaité ». ${ }^{4}$

\section{Enfin la sentence tombe :}

«Toutes les disputes entendues, Messieurs s'assemblent, et ordonnent que ledit veau sera prins et jetté dans le Rosne $» .^{5}$

\footnotetext{
${ }^{3}$ Anonyme, 1858 , p. 8.

${ }^{4}$ Ibid.

${ }^{5}$ Ibid., p. 9.
} 
En aucun moment, on ne voit en ce veau des traits pouvant mettre en doute ce que nous appellerions sa nature bovine. Sa forme rappelle suffisamment celle du veau pour que la question n'ait pas à être soulevée. La ressemblance joue un rôle proche de celui dévolu par nous à l'identité de nature. L'idée d'une nature spécifique transmise par les lois de la reproduction n'existe pas, ni même l'idée d'une observation attentive permettant de ranger cet être sous une catégorie spécifique. L'appeler " un veau » relève de l'évidence, et non du classement raisonné. De ce point de vue, seules les circonstances de la naissance de ce « veau » sont étonnantes : il est né du ventre d'une femme.

Pour l'immense majorité des personnes présentes, l'examen critique que nous appelons «observation » n'a pas lieu d'être : qu'apporterait-il de plus à l'évidence du prodige ? Ce prodige (le veau né d'une femme) suscite l'admiration, ainsi qu'un puissant étonnement mêlé d'effroi, et ce sentiment d'urgence suffit à motiver l'interprétation. Mais l'interprétation se suffit à elle-même : nul besoin de s'engager dans une description détaillée de l'apparence qui n'aurait guère de sens. Le narrateur comme les membres du conseil dont il rapporte les propos voient à l'évidence autre chose qu'un être humain. Ils ne voient pas davantage un monstre au sens où nous l'entendons, puisque son aspect ne pose pas de problème particulier, puisqu'il n'est pas un être apparemment interspécifique à ramener coûte que coûte à la déformation d'une forme propre à une espèce. Verraientils d'ailleurs un prodige humain comme un enfant à deux têtes que les causes de leurs craintes seraient sans commune mesure avec les nôtres. Jean Céard dans La Nature et les prodiges montre bien qu'ils le percevraient plus comme présage que comme relevant d'une commune nature humaine. ${ }^{6}$ Quant au veau né d'une femme, il n'a pas l'apparence d'un veau, il en est un, sans quoi la discussion entre les membres du conseil ne pourrait se poursuivre.

Même si une telle attitude n'excluait pas certaines descriptions plus précises, qu'utilisera encore plusieurs siècles plus tard Isidore Geoffroy Saint-Hilaire, fondateur de la tératologie scientifique, celles-ci font figure d'exceptions (la plus connue restant la description par Montaigne dans les Essais (Livre II, chapitre XXX) de l'enfant «pris et collé à un autre enfant sans teste $»^{7}$ ). Il ne fait pas de doute qu'elles entraient dans un tout autre contexte, par exemple comme moyen de montrer la différence radicale de vue entre Dieu et les hommes. En règle générale, le regard commun et le regard lettré ne s'aventurait pas aussi loin qu'il nous semble normal de le faire.

La question de l'humanité partagée ne se pose pas : elle n'intervient que subsidiairement dans le constat d'une naissance interspécifique. La modification du corps humain, racine de l'angoisse pour l'homme du $\mathrm{XX}^{\mathrm{e}}$ siècle, n'est même pas envisagée, car ce corps n'est pas même suspecté d'être un corps humain. Il est d'une autre nature.

Pour que la question de l'humanité puisse être au centre de l'hésitation perceptive face au monstre, encore faut-il qu'il y ait un sens à la poser, ce qui n'était pas le cas au début $\mathrm{du} \mathrm{XVII}^{\mathrm{e}}$ siècle. Dès lors que le prodige est un veau né d'une femme, son statut est clair, il redevient lisible et il échappe à l'ensemble des monstres humains qu'envisagerait la tératologie moderne d'un Geoffroy Saint-Hilaire.

\footnotetext{
${ }^{6}$ « De là vient qu'on les appelle monstres : non seulement parce qu'en raison de l'étonnement qu'éveille leur nouveauté les hommes se les montrent (monstrant), mais aussi parce qu'ils montrent (monstrant) que la colère de Dieu menace les hommes ». (Naucierus cité par Céard, 1996, p. 77 et p. 314).

${ }^{7}$ Le monstre de Montaigne n'a rien d'imaginaire : il serait classé parmi les monstres doubles parasitaires hétéradelphes selon les termes de la tératologie moderne.
} 
C'est au même Étienne Geoffroy Saint-Hilaire, fondateur de la science des monstres au début du XIX ${ }^{\mathrm{e}}$ siècle, que nous allons maintenant emprunter l'exemple le plus anciennement connu d'un authentique cas tératologique perçu comme transpécifique. Ce disant, nous suivrons la vision très «positive » que pouvait en avoir Geoffroy Saint-Hilaire. En l'absence de récit de première main, cet exemple nous apprendra autant sur le regard des tératologues du XIX ${ }^{\mathrm{e}}$ siècle que sur celui des égyptiens anciens, à l'origine de l'embaumement d'un monstre anencéphale.

\section{La momie monstrueuse}

Les anciens Égyptiens voyaient-ils certains monstres humains comme des animaux ? Une momie singulière, découverte au début du $\mathrm{XIX}^{\mathrm{e}}$ siècle par Passalacqua, un archéologue de Trieste, a pu le laisser penser. Elle se trouvait parmi une collection de momies de singes cynocéphales, vénérés par les Égyptiens, et enterrés dans les catacombes d'Hermopolis. Elle était accompagnée d'une amulette en terre cuite représentant un singe. Le récit de cette anecdote ouvre L'Histoire des monstres du médecin Ernest Martin, rédigée en 1880 (un demi-siècle après les travaux de Geoffroy Saint-Hilaire) : « Comme la taille et les dimensions dépassaient celles des animaux de ce genre, ensevelis dans les mêmes catacombes, on s'était demandé s'il n'y avait pas là quelque étrangeté et par conséquent quelque détermination à faire qui pût intéresser la science. On fit donc appel aux lumières d'Étienne Geoffroy Saint-Hilaire et c'est le résultat de son examen qu'il vint lui-même annoncer [lors de la séance du 9 janvier 1826 à l'Académie royale des sciences] » (Martin, 2002, pp. V-VII). Étienne Geoffroy Saint-Hilaire y reconnut en effet tout de suite un type de monstre humain qu'il avait abondamment étudié : le monstre anencéphale, dépourvu de cerveau et de boîte crânienne, dont les yeux globuleux saillent au-dessus de la tête sans front, comme coupée en deux à mi-hauteur. On avait tenté de lui extraire le cerveau par le nez, suivant le procédé habituel d'embaumement, preuve que l'on n'avait pas compris son caractère d'anencéphale (privé de cerveau). Les anciens Égyptiens auraient donc perçu cet être comme un animal né d'une femme, et l'avaient exclu des tombeaux humains. Mais laissons la parole à l'interprétation positive d'Étienne Geoffroy Saint-Hilaire :

«Tite-Live, Valère-Maxime, Pline, etc., parlent de femmes qui, par des enfantements extraordinaires, donnaient lieu aux plus sinistres présages, obligeaient de recourir à des lustrations, à des purifications générales : c'était quand elles accouchaient d'êtres caractérisés singes ou éléphans par les formes bizarres de leur tête. Ces prétendus mammifères singes ou éléphans ne sont pour moi que des monstruosités humaines des genres que j'ai déterminés sous les noms d'Anencéphales et de Rhinencéphales [ces derniers sont des monstres réels nés avec une sorte de trompe et un seul œil], mais c'était là une présomption, une déduction de quelques faits, qu'il est sans doute intéressant de changer en certitude.

Cet avantage nous est procuré par la momie possédée par M. Passalacqua. C'est plus qu'un document historique, fourni par cette sorte de mammifère-singe, que la circonstance de son exclusion des sépultures humaines : or, cet être d'une nature ambiguë a été découvert dans des catacombes réservées aux animaux, dans des caveaux où se trouvaient en particulier des singes. Et de plus, ce qui montre qu'il n'y avait là ni méprise, ni ignorance, mais qu'on observait en cela un rite religieux, c'est une amulette suspendue au cou de la momie. Cette amulette, faite en terre cuite ou en mauvaise porcelaine, est une exacte copie du singe cynocéphale (...). La forme de ce symbole exprime-t-elle l'intention d'une comparaison entre l'infériorité organique 
accidentelle de la monstruosité embaumée, et l'infériorité normale de l'être le plus dégradé parmi les animaux à face humaine ? Ceci est une conjecture ; mais ce qui n'en est pas une, c'est que les amulettes en collier étaient dans la vieille Égypte un attribut réservé aux hommes » (Geoffroy Saint-Hilaire, 1826, pp. 3-4).

Nous serions tentés de suivre Geoffroy Saint-Hilaire à ce sujet, puisqu'il avait fait partie de l'expédition en Égypte de Napoléon et s'appuyait sur une bonne connaissance des coutumes antiques. Si son interprétation est exacte, il semblerait donc que l'être momifié n'ait été assimilé ni à un animal ni à un homme, mais à un être intermédiaire, hautement fascinant pour des Égyptiens anciens qui croyaient à la métempsychose où, au sortir de la mort, homme et animal se fondent l'un en l'autre. Un être qui évoquait le divin pour un peuple dont les Dieux sont des hybrides à têtes thériomorphes sur des corps anthropomorphes, un être enfin qui ne pouvait que profondément toucher un peuple vouant un culte à la mort : « quel plus grave sujet de méditation et d'entraînement mystique, que le spectacle d'un être en quelque sorte voulu à-la-fois et délaissé par la nature, tenu de naître et de mourir au même moment ! » (Geoffroy Saint-Hilaire, 1826, pp. 3-4). Un être intermédiaire entre l'homme et l'animal, entre les vivants et les morts, méritait donc amplement d'être momifié pour rejoindre l'éternité.

Bien qu'ayant fondé la tératologie explicitement contre ces assimilations abusives entre l'homme et l'animal, Étienne et Isidore Geoffroy Saint-Hilaire leur ont toujours accordé un grand intérêt. Pour arracher le public à l'obscurantisme, encore faut-il se placer sur le terrain même des représentations que l'on entend combattre, comme le fait Étienne Geoffroy Saint-Hilaire dans le récit suivant, où il est question de l'accouchement d'un nouveau-né du même type que le monstre momifié cité plus haut :

« On s'attendit à un accouchement laborieux, et, pour avoir à y procéder avec plus de facilité, on fit prendre à la malade une position particulière sur le bord du lit.

Cependant on se dispose : une élève approche une lampe du lieu de la scène, quand tout à coup le fœtus prend de lui-même son départ. Son mouvement est rapide : il est lancé, il saute, m'at-on rapporté, sur la lampe qu'il éteint ; mais il fait mieux, car il arrive avec d'amples bagages : il est suivi de son placenta, que la sage-femme, qui est à ce moment dans l'obscurité, reçoit dans son autre main, sans trop savoir ce que signifie cette nouvelle évacuation. L'embarras de la sage-femme augmente encore par l'épanchement de nouvelles eaux; car tout échappe à la fois du sein maternel ; tout arrive sur elle, ses deux mains soutenant chacune ce double produit de l'enfantement.

Mais voilà bien d'autres sujets de surprise : du moment qu'elle eut remis son principal dépôt dans une autre partie de la salle, éclairée et préparée pour recevoir l'enfant, cet être, qui vient de faire une si brusque entrée dans le monde, regarde fixement les assistans. C'est un monstre à traits hideux; sa tête est enfoncée dans les épaules et renversée en arrière ; ses yeux sont saillans et ouverts, le crâne est déprimé, rompu et concave, le museau avancé ; le cou manque, et le dos est large et pelé ; enfin, son attitude, toute sa physionomie ne rappellent et ne donnent l'idée que d'un crapaud. On ne se défendit pas d'un peu d'effroi, et l'on ne se rassura que lorsque la réflexion eut ramené à l'idée d'un bien moins grand désordre. Quelqu'un s'écria : C'est un singe, une manière de singe!

Mais, l'esprit revenu de tant de surprises, on connut que c'était une fille mal conformée dans toutes les parties postérieures du tronc et de la tête, une fille Anencéphale. Ses voies aériennes étaient obstruées, et elle termina, dans le milieu atmosphérique, après deux ou trois mouvemens spasmodiques, sa vie passée dans l'utérus ; n'ayant ainsi reçu, au commencement de toutes choses pour elle, qu'une viabilité semblable à celle des poissons, des conditions organiques, pour exister seulement dans un milieu aquatique. » (Geoffroy Saint-Hilaire, 1824, pp. 3-4). 
Dans ce passage digne d'un roman gothique, Étienne Geoffroy Saint-Hilaire prend en considérations les assimilations communes à un crapaud, puis à un singe, qu'il s'agit de dépasser en ramenant le monstre à une régularité : il s'agit d'une fille anencéphale. La comparaison entre le fœtus capable de vivre uniquement dans les eaux du ventre maternel et un poisson ne relève pas pour lui d'une analogie, elle interdit de dévaloriser le monstre en raison de son incapacité à vivre une vie extra-utérine. La comparaison est d'autant plus convaincante pour Geoffroy Saint-Hilaire qu'il croit les fœtus capables de respirer par les pores de leur peau dans le liquide intra-utérin. On le constate : les associations jugées probantes ne sont au fond que celles qui reposent sur une théorie acceptée et considérée comme scientifique. Ainsi Geoffroy Saint-Hilaire rapproche fréquemment l'organisation des monstres humains de celles des animaux, car il y voit la confirmation de sa théorie d'unité de composition organique, selon laquelle tous les vivants animaux partagent un même plan d'organisation. Il y a continuité entre les formes animales, et toute déformation reconduit nécessairement vers une autres forme déjà connue. Cela autorise Geoffroy Saint-Hilaire à de nombreuses comparaisons qu'un biologiste contemporain jugerait à son tour purement associatives ${ }^{8}$.

Malgré la limite que rencontre toute interprétation, y compris scientifique, la science positive de la fin du $\mathrm{XIX}^{\mathrm{e}}$ siècle s'est montrée beaucoup plus cavalière et conquérante que Geoffroy Saint-Hilaire dans le passage cité plus haut. Georges Canguilhem dénonce ce réductionnisme de l'interprétation dans son article «La Monstruosité et le monstrueux $»{ }^{9}$. Nous allons montrer l'inadéquation de ce réductionnisme aux représentations courantes avec un exemple emprunté cette fois à l'Antiquité grecque.

\section{La naissance d'un jeune centaure}

Ernest Martin dans son Histoire des monstres de 1880 rapporte l'anecdote suivante : Périandre ayant convié à un festin ses amis philosophes, dont Dioclès et Thalès, ils se retrouvèrent en présence d'un jeune centaure qu'une jument venait de mettre bas : un jeune pâtre apporte alors « un petit tendron, qu'il disoit estre né d'une jument, duquel le hault jusques au col et aux mains avoit forme humaine et tout le reste de cheval, cryant au reste ne plus ne moins que les petits enfiants quand ils sortent du sein de leur mère. » (Martin, 2002, p. 38). Dioclès y voit un « présage de discord et de sédition », Thalès suppose quant à lui un commerce entre le jeune pâtre et la jument. Mais ces interprétations ne remettent pas en cause l'évidence du fait de la naissance d'un hybride interspécifique.

Face à de tels témoignages, les positivistes du XIX ${ }^{\mathrm{e}}$ siècle rapportaient l'enfant centaure à un dédoublement partiel de l'arrière-train, une malformation que l'on rencontre dans l'espèce humaine. De nombreuses thèses de médecine tentent aujourd'hui encore de lire de tels témoignages à travers cette réduction à une donnée préalablement connue ${ }^{10}$.

\footnotetext{
${ }^{8}$ Étienne Geoffroy Saint-Hilaire (1825, pp. 4-5) comparait ainsi l'organisation génitale d'un monstre Aspalasome à celle de la taupe femelle. Son " pied amaigri et allongé » dont le tarse " oscillait sur le péroné » paraît « presque la répétition d'un conformation particulière au paresseux, dit unau ».

${ }^{9}$ « L'anthropologie positiviste s'attache à déprécier les mythes religieux et leurs représentations artistiques. En 1878, le Dr Parrot cherche à établir, devant les membres de la Société d'anthropologie, que le dieu nain Phtah, adoré par les Egyptiens, reproduisait les caractéristiques d'un monstre achondroplasique » (Canguilhem, 1971, p. 181). ${ }^{10}$ Citons quelques tentatives de médecins et biologistes pour ramener les formes artistiques dans le giron des formes tératologiques: Jeannerey (1923, pp. 131-138); Fricaud (1933); Bressou (1944); Bertin (1950); Carneiro Alves (1987); Wolff-Quenot (1996).
} 
Mais la prégnance de l'image de l'enfant centaure ne vient pas seulement d'une naissance tératologique un jour donné. On pourrait d'ailleurs affirmer contre le regard positif que c'est le centaure mythologique qui explique la vision de ce centaure réel et non l'inverse : l'effet de cette naissance est d'autant plus marquant que la forme de l'hybride supposé est perçue au travers d'une grande figure typique de l'imaginaire qui oriente le regard. La connaissance d'une forme familière modèle l'acte perceptif et influence cette reconnaissance supposée.

Intéressons-nous davantage à la figure du centaure. Son pouvoir de fascination vient également de ce qu'elle s'appuie sur une expérience vécue : le centaure figure l'entente parfaite entre le cavalier et son cheval. Ce dernier réagit comme un prolongement du corps du cavalier qui peut par la seule pensée initier un mouvement. Par la figure du centaure qui fusionne l'homme et le cheval, le cavalier retrouve une expérience familière du corps vécu, la métamorphose de son propre corps dans la relation qui le lie à sa monture.

Nous avons conscience en proposant une telle interprétation de substituer une explication psychologisante à une explication réductionniste de type anatomo-pathologique. Mais une telle interprétation a l'intérêt de ne pas enfermer dans de très étroites limites la figure du centaure. Et sans doute le vécu du corps à cheval était-il plus familier pour les auditeurs de l'Antiquité que ne l'était une vision anatomique du corps difforme. Se fondre dans le corps d'un animal en le chevauchant n'est pas seulement une réinterprétation contemporaine d'une expérience passée. Elle fait appel à une idée de continuité entre l'homme et l'animal à laquelle la cosmologie et la mythologie grecque étaient beaucoup moins réticentes que nous ne le sommes.

La figure de l'hybride comme manifestant la fusion entre deux êtres possède d'autres prolongements fantasmatiques : elle renvoie à une sexualité zoophilique transgressive, comme celle qu'évoquait Thalès dans le passage précédent. Le commerce entre l'homme et l'animal peut conduire à des naissances inattendues qui mettent en évidence des désirs de fusion plus intimes et difficiles à reconnaître en dehors de ces fruits supposés de la copulation.

Ces motivations fantasmatiques permettent de comprendre la permanence de telles représentations, alors même qu'Aristote avait depuis longtemps déjà fermement nié la possibilité des hybrides ou d'êtres transpécifiques par la seule considération des temps de gestation des différentes espèces ${ }^{11}$.

Ce décalage important entre représentations raisonnées et représentations fantasmées se repère à toutes les époques : certaines idées d'hybridation ont survécu longtemps, même après le début de la science des monstres, comme vont nous le montrer quelques exemples empruntés à l'époque de l'exhibition industrielle du corps monstrueux, la fin du XIX ${ }^{\mathrm{e}}$ siècle en Europe et surtout aux États-Unis. Pourra-t-on y retrouver, à des siècles de distance, la même prégnance des grandes figures de l'imaginaire?

\footnotetext{
11 «Mais la naissance d'un pareil monstre, d'un animal dans un autre, est impossible : on le voit par la durée de gestation qui est tout à fait différente chez l'homme, le mouton, le chien, le bœuf ; or, il est impossible que chacun d'eux naisse en dehors de son temps normal de gestation. " (Aristote, De la Génération des Animaux, IV 3,769$)$
} 


\section{Fantasmes à l'époque contemporaine : les grandes exhibitions de la monstruosité au XIX ${ }^{e}$ siècle}

Les appellations suggestives évoquant une hybridation foisonnaient au moment des grandes exhibitions de phénomènes humains: Sans prétendre donner une liste exhaustive, citons les hommes-chiens au visage velu, la femme-hyène, la femme-ours, la femme-limace privée de squelette sur tout le corps excepté la tête, l'homme-escargot, la femme-jument, la femme-tête de mule, la femme-pingouin aux membres atrophiés, les femmes-homards aux mains fendues en deux immenses doigts, les hommes ou femmeséléphants (le plus célèbre «Elephant Man» étant Joseph Merrick dont plusieurs livres ont raconté l'histoire ${ }^{12}$ ). Très loin d'être toutes motivées par une assimilation visuelle convaincante, ces dénominations étaient révélatrices d'un effort pour satisfaire les fantasmes du public (notamment les fantasmes sexuels, sévèrement réprimés à la fin du $\mathrm{XIX}^{\mathrm{e}}$ siècle, que le corps monstrueux permettait d'aborder à moindre frais). Ces exhibitions profitaient de la crédulité du public populaire pour réintroduire dans les livrets et les discours de présentation des phénomènes l'idée d'une genèse bestiale, ou d'une influence de l'imagination de la mère sur le fœtus.

Face à l'écho imaginaire que rencontre une forme évoquant l'hybridation, la gravité d'une affection compte peu: des réactions très fortes peuvent être suscitées par des anomalies très simples et très localisées, comme l'inversion des rotules. Les personnes atteintes de cette malformation plient le genou vers l'arrière et non vers l'avant. L'angle ouvert que dessine la jambe inversée évoque la patte arrière au talon haut placé des animaux digitigrades (comme le chien) ou onguligrades (comme la vache). Cette apparence d'animalité conduit à la représentation composite d'un arrière-train animal monté, par exemple, sur le buste d'une belle jeune fille, telle Lucy Elvira Jones, née en 1881 qui se produisait dès l'âge de treize ans au Texas au titre de « femme-chameau ». Son impresario, habillé en Arabe, la faisait s'accroupir très lentement à la manière d'un dromadaire, alors qu'un phonographe diffusait une musique orientale. Le but de cette mise en scène grossière n'était sans doute pas de faire croire à l'hybridation, mais de satisfaire les fantasmes du public, un public ravi d'imaginer à quoi ressemblerait sans vêtement cette femme-chameau orientale, ce qui était un bon moyen détourné de parler de sexualité, ordinaire ou bestiale.

R. Bogdan (1988) a abondamment souligné l'importance de la mise en scène dans l'exhibition des monstres: l'étrangeté et l'ambiguïté interspécifique ne relèvent pas uniquement de la malformation ou de la différence de l'individu exposé, mais de l'orientation du regard induite par le contexte d'exhibition. Ces jeux sur l'apparence d'hybridation sont donc à la fois involontaires et volontaires: involontaires, parce qu'ils dérivent d'une tendance de l'esprit à repérer d'abord des formes familières, fussent-elles fantastiques ; volontaires, parce que les exhibitions exploitaient cette tendance et s'efforçaient par tous les moyens de les prolonger.

En voici un nouvel exemple. À la même époque, on pouvait voir dans les mêmes lieux d'exhibition un gros chien habillé en homme laper dans sa gamelle. Soudain, le public le voyait s'étirer, puis se redresser, prendre avec ses pattes une pipe puis se l'allumer tranquillement avant de s'asseoir dans un fauteuil. Pendant un instant, il proposait au public un authentique mélange entre l'homme et le chien, une transformation visible

${ }^{12}$ Voir, entre autres, Montagu, 1996. 
de l'un en l'autre. Adrian Jeftichew, l'homme ainsi exhibé, était atteint d'hypertrichose, une variation fonctionnellement sans gravité, entrainant l'apparition de longs poils sur tout le visage. Ce lieu d'identification privilégié de l'être humain devient alors inintelligible. L'habitude de se repérer dans ce visage faisant défaut, l'expression caractéristique de l'humain disparaît sous le masque de l'animalité. Voilà qui permettait de donner à voir des «hommes-chiens" ou «hommes-loups », échos des légendes de peuples cynocéphales ou de la métamorphose des lycanthropes.

En effet, l'image de l'homme-loup comme celle du centaure étudiée plus haut possède un enracinement anthropologique. Paul Schilder insiste à propos des métamorphoses de l'image du corps sur ce fantasme à la fois individuel et collectif : « Nous savons que les psychotiques ont souvent la sensation d'être transformés en animal, un chien ou un loup par exemple », et l'on retrouve ce fantasme de métamorphose dans « les contes de fées, et mythes de tous les peuples » (Schilder, 1968, p. 222). Précisons que s'il y a bien au sein de l'humanité une tendance à l'autoplastie imaginaire, celle-ci prend une valeur fort différente selon les peuples et les cultures. Mais cela n'enlève rien à l'intérêt de cette tendance à la déformation corporelle que l'on rencontre dans tant d'histoires fantastiques peuplées de monstres composites. L'autoplastie imaginaire se donne pour Schilder comme une « activité d'essai continuelle » (Schilder, 1968, p. 227), passant notamment par la tendance à se représenter humain-animal. Il s'agirait là d'une forme d'expression médiatisée de la violence des pulsions. Car l'animalité renvoie davantage à la face sombre de l'humain qu'à une caractéristique réellement animale: le « loup » qui se mélange à l'homme n'a pas une cruauté animale, mais une agressivité bien humaine qui a faim de sa faim future. Il correspond au besoin d'exprimer une altérité en soi. La figure d'un homme-animal donne corps aux sentiments d'agressivité et de cruauté. Ce n'est donc pas un hasard si l'autoplastie choisit cette forme mythique pour s'exercer, car, réciproquement, cette figure typique de l'imaginaire humain s'enracine dans un vécu du corps et des affects. S'il existe des inventions individuelles dans le monde des formes, celles-ci nourrissent et se nourrissent de référents culturels préexistants. Il y a là un phénomène de genèse réciproque pour reprendre une expression de Gilbert Durand ${ }^{13}$.

En vertu de motivations d'ordre anthropologique comme le désir de variation du corps dont nous venons de parler, l'hybridation, dans son aspect statique de forme déjà aboutie, ne saurait être nettement distinguée de la métamorphose dans son aspect dynamique. En découvrant un hybride apparent, chacun réinvente sa propre métamorphose ${ }^{14}$.

La fascination pour les hommes-loups apparents ne vient donc pas seulement de ce que les figures lycanthropiques sont déjà connues comme formes mythologiques: elles s'ancrent dans l'expérience d'une altérité pulsionnelle en marge de l'humanité socialement affichée. C'est en satisfaisant un désir familier d'autoplastie que l'individu atteint d'hypertrichose devient aussi frappant pour le public. Sans cet arrière-plan anthropologique, il ne serait jamais qu'un homme très velu, et cette simple variation de surface ne serait pas perçue comme une monstruosité.

\footnotetext{
${ }^{13}$ Cette expression est utilisée par Gilbert Durand pour désigner l'échange perpétuel dans l'imaginaire entre les pulsions subjectives et les intimations objectives du milieu social (1992, p. 38).

${ }^{14}$ Pour plus de détails à ce sujet, nous renvoyons le lecteur à notre ouvrage Phénoménologie des corps monstrueux, 2006.
} 


\section{Les craintes contemporaines associées aux corps chimériques}

L'analyse de ces différents exemples semble donc montrer que les explications rationalisantes ne touchent pas leur véritable objet. Elles réduisent le regard sur le monstre à une méprise en occultant l'influence des grandes figures de l'imaginaire humain. Cela est vrai non seulement à des époques où ce que nous nommons « merveilleux » ou « fantastique » n'était pas nettement distinct de ce que nous nommons « réel», mais encore à l'époque contemporaine, lorsque le monstrueux réel rencontre ces figures anthropologiques de l'imaginaire. Il serait illusoire de penser que nous sommes aujourd'hui définitivement libérés de telles représentations parce que nous connaissons l'existence d'une science des monstres, parce qu'une pensée rationnelle est venue se substituer à une pensée analogique ou magique. Il suffit pour s'en convaincre de penser à nos propres réactions à propos de la création de chimères réelles expérimentales entre la caille et le poulet, entre le mouton et la chèvre ${ }^{15}$.

Ainsi, nous sommes loin d'être certains de l'impossibilité d'une naissance interspécifique entre humains et grands singes, d'autant que des tentatives de fécondations ont déjà été menées par le passé. S'il venait à naître, cet être serait-il humain, ou bien resterait-il animal ? Il viendrait brouiller nos catégories apparemment si bien établies en ce qui concerne la distinction entre l'homme et l'animal sur le plan moral et juridique. Tous les fantasmes d'hybridation reparaissent alors, sous-jacents à ces interrogations plus conformes à une argumentation rationnelle.

On retrouve ces craintes dans les réactions aux projets de xénogreffe thérapeutique : pour suppléer au manque d'organes humains, il est envisageable de greffer à leur place des organes de porcs génétiquement modifiés. Les personnes ainsi greffées pourraient donc se percevoir comme des chimères composites, et l'on jugera de la difficulté à vivre son corps s'il est partiellement un corps de porc : toutes les difficultés rencontrées par les greffés (la présence d'une partie d'un autre en soi, les risques de rejets psychologiques) pourraient être ici amplifiées. Inversement, les porcs génétiquement modifiés changeraient de statut, en tant que faisant potentiellement partie du corps humain : leur viande resterait-elle consommable ? Manger leur chair, cela ne pourrait-il pas être vécu comme un acte cannibalique ?

Face à cette absence de certitude générée par le progrès du savoir et de la technique, l'activité fantasmatique stigmatisée plus haut reparaît de plus belle. Il suffit de la réalité d'une expérience pour relancer le travail de l'imagination. Chaque modification génétique d'un animal par un gène humain fait resurgir le même type d'idées, même lorsqu'elle n'a comme conséquence que de faire produire du lait maternel humain par des bovins ou des ovins.

\section{Naissances et genèses bestiales}

On le voit : ce que nous pourrions qualifier de «pensée magique » pour décrire cette impression d'une genèse transpécifique ou interspécifique reste bien présent aujourd'hui

${ }^{15}$ Citons par exemple Le Douarin, 2000. 
dans le cadre de nos représentations non verbalisées. Lorsque l'intensité des affects est trop importante, comme lors de la naissance d'un enfant malformé, de telles idées resurgissent spontanément. Ainsi, à l'annonce de la naissance d'un fils gravement handicapé, l'idée « d'un monstre innommable - doté peut-être d'une tête de chat et d'un corps gonflé comme un ballon » vient à l'esprit de Bird, le narrateur de Une Affaire personnelle, roman à caractère autobiographique de Kenzaburo Oé (1968, pp. 24-25). Lorsqu'il montre le bébé pour la première fois à une amie, il ajoute : « Il ne ressemble à personne. Il n'a même pas l'air humain. » (Oé, 1968, p. 161).

Cette inquiétude irrationnelle quant à la genèse concerne non seulement l'enfant déjà né et l'enfant à venir, mais également celui que l'on a été, ce moment caché de l'histoire personnelle où le corps n'était encore qu'une ébauche. Pour l'enfant ou l'adolescent atteint d'un handicap, l'idée d'une genèse fabuleuse peut servir à recouvrir l'inacceptable et le non-dit de la différence. Le jeune Henri Cueco parle ainsi du secret et du silence familial observé à propos de sa difformité faciale (une fente palatine) jusqu'au jour où un médecin lui dit en lui tapotant la joue : "quel joli bec de lièvre ! ». Ces termes, que sa famille refuse de lui expliquer, le renvoient à l'idée d'une genèse hybride de soi, à une sexualité perverse qui l'aurait mis au monde par la transgression : «à la faveur des ombres de mon livre d'histoire, vers l'âge de treize ans, je crus que j'étais le produit d'une honteuse pratique zoophilique qui avait uni dans la profondeur des futaies druidiques mon ancêtre avec un lapin $\gg^{16}$.

Dans cet exemple, la représentation fantasmatique propose une solution à un problème inacceptable, générateur d'une angoisse qu'aucune compréhension rationnelle ne peut soulager : la genèse incompréhensible d'un visage anormal. La pensée magique fait alors irruption dans la conscience pour soulager l'incertitude associée à cette impossible quête d'une identité normale, chez cet enfant qui se sent en permanence en décalage identitaire.

\section{Conclusion}

Nos représentations contemporaines sont donc loin d'être libérées des représentations d'êtres hybrides et transpécifiques, surtout face aux angoisses liées aux manipulations génétiques, à la naissance présente d'un enfant handicapé, à la genèse d'un handicap actuel. En ce sens, les approches phénoménologique (au sens de Gilbert Durand, 1992), psychologique et psychanalytique peuvent nous venir en aide, comme moyen de saisir l'émergence de ces représentations. Comme les autres approches citées plus haut, elles renseignent sur une manière de penser contemporaine autant qu'elles nous apprennent sur leur objet, ce qui n'autorise ni à les surestimer comme universalisables, ni à les disqualifier comme abusives. Ces derniers exemples ont eu à tout le moins le mérite de nous montrer une certaine continuité au sein de l'histoire des représentations du corps monstrueux et hybride, sans négliger toutes les réserves précédemment énoncées sur l'idée de représentations atemporelles de la monstruosité, bien que les perceptions diffèrent en nature, bien que la fonction des images soit variable, certaines figures anthropologiques demeurent, au rang desquelles celle du monstre hybride qui mêlera pour longtemps encore dans nos esprits l'homme et l'animal.

\footnotetext{
${ }^{16}$ L'Ane, avril 1995, nº 60, pp. 25-28. Cité par Serge Tisseron, 1996, pp. 45.
} 


\title{
Chapitre 5 \\ Quelques doutes sur la différence entre l'homme et l'animal
}

\author{
Pierre Guenancia
}

On définit l'homme comme un animal qui aurait, en plus de ce qui est commun aux autres animaux, une propriété ou une faculté qui l'en différencierait et qui l'aurait conduit ainsi à un dépassement irréversible de l'état animal. En ayant quelque chose de plus que les autres animaux, l'homme serait devenu autre qu'eux. Ainsi de la faculté de rire, de la faculté de penser ou de raisonner, de la faculté de s'associer, non sous la forme d'une bande, d'un groupe, ou d'une famille, mais dans celle d'une société politique. La liste est loin d'être close, chaque différence engendrant une infinité d'autres différences, chacune exhibant pourtant un trait spécifique et distinct de l'homme. On n'en finirait pas de recenser toutes les définitions qui prétendent être les seules à enseigner la différence spécifique de l'homme avec l'animal, bien qu'aucune d'elles ne puisse être, à un niveau ou à un autre, sinon contestée, du moins relativisée. Mais ce travail fastidieux serait de plus inutile. La plupart des définitions traditionnelles reviennent à dire que l'homme possède quelque chose qui, en s'ajoutant à sa nature animale, la transforme essentiellement et, à la limite, la dénature.

Ainsi envisagées, ces définitions signifient que l'homme est le seul animal qui ne soit pas un animal. Ce que l'on sait déjà, si l'on emploie dans la définition le terme qu'elle cherche à définir ! Aucune définition ne nous permettrait de reconnaître l'homme parmi la multitude des espèces animales, si nous ne le savions déjà d'une façon indéfinissable. Le but de telles définitions est à l'évidence autre : il consiste à s'assurer que l'homme possède une place bien à lui et qu'il est seul à occuper. Ce souci ne semble pas être celui de l'animal qui paraît plutôt chercher à « animaliser » l'homme en l'intégrant dans sa propre hiérarchie et en lui faisant jouer le rôle d'un congénère (le phénomène est décrit par Lorenz sous le nom d'imprinting ${ }^{1}$ ).

\footnotetext{
${ }^{1}$ Cf. Lorenz, 1975, p. 107 et p. 110 ; Tinbergen, 1979, p. 143. Canguilhem (1970, p. 123) écrit pour sa part : «...l'homme est perçu par l'animal comme animal-stimulus, congénère, associé ou ennemi [...] L'animal perçoit l'homme en l'animalisant et, par exemple, en l'incorporant à sa hiérarchie sociale ».
} 
Le moins que l'on puisse dire, c'est que l'animal n'est pas payé de retour : on ne le grandit que pour mieux rabaisser l'homme, et les arguments dont on se sert alors ne sont jamais que des arguments ad hominem. Moralistes et humanistes ne se réfèrent à l'animal que pour lui demander de montrer qu'il n'a pas, selon les cas, les qualités de l'homme ou ses vilains défauts. L'homme est tellement peu sûr de savoir vraiment ce qu'il est qu'il presse l'animal de le lui « dire » et cherche dans cette altérité de quoi déduire sa propre identité. La conscience de son identité personnelle ne paraît pas lui suffire, il lui faut aussi celle d'une appartenance à une espèce distincte de toute autre qui conférerait à ses ressortissants le sentiment d'une identité commune et transindividuelle. II me paraît douteux que la conscience de soi comme ego trouve son fondement dans le fait d'être un homme, ou qu'elle en dérive par différenciation progressive. Ces deux formes de conscience ne me semblent ni devoir coïncider dans une expérience élargie, ni pouvoir présenter la même certitude.

L'idée que les lignes qui suivent vont développer est qu'il y a une différence essentielle entre le fait métaphysique de se connaître comme moi ou ego et le fait empirique de se reconnaître comme homme. Certes, tout homme est un être capable de dire « je »; mais c'est pourtant seulement quand un homme dit « je » (et s'adresse par là même à un autre) qu'il actualise la forme jusque-là vide de l'homme dans laquelle il peut se projeter et se reconnaître, mais de laquelle il est nécessairement distant, du fait même de la singularité constitutive de cet acte (Benveniste, 1966, chap. XX). Ainsi l'ego peut-il se rapporter à la forme ou à l'idée indéterminée d'homme comme à un devoir-être, et non comme à son être-propre. En ce sens, l'idée d'homme ne peut être séparée de celle de norme, voire du normal, sans que le contenu de cette norme se laisse définir par les traits caractéristiques de l'espèce à la manière d'une définition naturaliste. Seul un être qui dit « je » peut, en se distinguant ainsi fondamentalement de son espèce, constituer son identité sur le mode de ce qu'il veut ou doit être, et en faire le pôle régulateur de son individualité. Seul un être qui se pense comme sujet peut avoir son identité devant lui, et non derrière lui. La question du sens de l'humain n'est pas la question de l'origine de l'homme et ne saurait s'y réduire, pas plus que le problème de la reconnaissance de l'homme ne s'explique par le recours aux propriétés communes et caractéristiques de l'espèce humaine. Nous ne chercherons donc pas dans les remarquables études contemporaines sur le comportement animal des preuves ou des arguments, mais seulement des exemples dont il n'est, après tout, pas interdit de se servir philosophiquement, comme le montre notamment l'œuvre de Konrad Lorenz dont ce n'est pas là le moindre intérêt, ni la plus petite de ses audaces spéculatives.

\section{L'homme : un être qui dit « Je »}

Le langage peut être envisagé selon deux directions : l'une qui en fait une propriété acquise à un moment du développement phylogénétique de l'homme et se transmettant par une tradition culturelle qu'il a rendue possible ; l'autre qui en voit la «naissance » et l'actualité dans le discours grâce auquel un homme fait entendre sa pensée à un autre et aussi à lui-même. Tel est, on le sait, le critère cartésien de la distinction de l'homme avec l'automate animal-machine, ou simulacre de l'homme. Descartes ne mentionne pas la langue, au double sens d'organe phonique et d'ensemble de règles d'utilisation du langage, mais la parole, ce moment où le langage s'incarne pour ainsi dire dans un corps qui passe ainsi de la gestualité ou de la gesticulation à la signification. L'autre homme est donc un homme comme moi, dans la mesure où je le comprends et me fais comprendre 
de lui. La compréhension réciproque fait des hommes des mêmes hommes. Rien de tel entre l'homme, fut-il « hébété », et l'animal, si supérieur soit-il. C'est d'emblée, et non au terme d'une expérience, que le face à face avec l'animal confronte l'homme à une irréductible altérité ${ }^{2}$. La curiosité que le petit enfant éprouve envers l'animal est l'une des expériences les plus manifestes de cette obscure conscience d'altérité.

Plus tard même, l'on n'observerait sûrement pas avec la même attention mêlée d'étonnement les jeux et les comportements animaux si l'on ne pressentait pas l'imprescriptible limite de ce spectacle, le fait qu'il est destiné à demeurer un spectacle, et nous un spectateur. L'animal nous maintient en dehors de lui-même, il circonscrit les limites d'une extériorité dans laquelle nous savons ne pas pouvoir pénétrer. La perception du corps de l'animal, de l'animal comme corps, coïncide avec le sentiment de cette extériorité infranchissable. L'animal donne à voir son corps comme la seule chose qu'on peut voir de lui. Son corps n'est pas seulement la découpe matérielle de son individualité, il est le seul objet sur lequel se pose le regard qui est comme réfléchi par ce corps qu'il observe mais ne traverse pas. Pour que le regard de l'homme s'applique au corps de l'homme, il faut une sorte de réduction de l'attitude naturelle qui se convertit en regard clinique, esthétique ou désirant. II se produit, dans ces expériences comme dans le face à face avec l'animal, une sorte de disjonction entre altérité et compréhension, comme si l'altérité ne pouvait apparaître vraiment que là où cesse la compréhension de l'autre. C'est pourquoi l'autre homme n'est jamais une altérité radicale, sauf à la constituer intentionnellement par un regard « indiscret», une altérité radicale, mais un autre homme, un être générique en même temps qu'un individu.

II ne s'agit pas ici de relation dialectique, ni de reconnaissance d'une conscience par une autre dans la rivalité et la lutte. L'homme ne reconnaît pas l'autre parce qu'il en a peur, mais parce qu'il se fait comprendre de lui. Ce rapport immédiat de compréhension est inséparable d'une relation confiante, tellement confiante que l'homme commence à s'abandonner à l'autre, et que la première relation de l'un à l'autre est une impérieuse demande de compréhension. L'acquisition du langage permet à l'homme d'assouplir et de relativiser cette demande, car à partir du moment où l'homme parle, il dispose de la possibilité de se comprendre lui-même et devient de ce fait moins exigeant envers les autres. Mais avant le langage, il y a la compréhension, dont le registre est l'expression. Le corps humain, on l'a souvent dit, est un corps expressif, un lieu où prennent corps les significations. Le corps humain est un corps immédiatement traversé par la signification, et c'est ce qui le rend visible et transparent à l'autre, c'est ce qui fait qu'on ne s'arrête pas au corps comme à un dehors; c'est aussi ce qui fait que l'homme, d'emblée, a un corps. Il n'est pas son corps, comme l'animal dont c'est pour ainsi dire la carapace et l'identité même. Si l'animal, comme on le remarque souvent, sait se servir très vite de son corps, à la différence de l'enfant, c'est parce qu'un rapport d'inhérence ne nécessite aucun apprentissage, de la même manière qu'une machine est prête à fonctionner aussitôt construite. Avoir un corps requiert au contraire d'en apprendre l'usage et, par conséquent, d'imiter ce que l'on voit faire chez l'autre.

Ainsi, le rapport à l'autre homme est ce qui s'interpose toujours entre l'homme et les choses pour en instruire l'usage. Si l'enfant a tout à apprendre des hommes, ce n'est pas parce que l'homme est un animal social (car il ne semble pas que les autres animaux

\footnotetext{
${ }^{2}$ Nous reviendrons plus en détail sur cette question dans la quatrième partie de cette étude.
} 
sociaux apprennent grand-chose les uns des autres), mais parce que l'homme a, d'entrée de jeu, une relation d'entente avec les autres, et qu'à celle-ci se subordonnent toutes les autres. Son corps n'est que le moyen par lequel il s'adresse à l'autre, il est l'instrument, ou le support matériel, de la signification. Si le sens n'était pas strictement contemporain de la vie humaine, on ne voit vraiment pas comment il pourrait parvenir à s'y fixer et la pénétrer si intimement. L'union de l'âme et du corps est, sous cet angle, union du sens et du corps, ou bien, car cela revient au même, union de l'homme avec l'autre. L'âme est le corps animé par une intention de signification ou un vouloir-dire. L'âme n'est pas principe de conservation, principe vital, mais volonté de signification ; elle n'est pas effort, mais intention ou volonté. Et c'est bien pour cela que l'homme est d'emblée union de l'âme avec le corps et non corps d'abord, âme ensuite.

Le souci de soi - de la conservation de soi - ne nous paraît donc pas la caractéristique essentielle de l'humain, alors même qu'on y verrait la détermination fondamentale de l'être vivant. Il y a une inconscience du souci de soi et de la conservation de son être qui nous semble incompatible avec la conscience qu'implique au contraire le fait de signifier à l'autre, d'une manière plus globale que précise, qu'il est compris parce qu'il me comprend. Cette ouverture ne peut jamais se clore, alors que le souci de soi conduirait nécessairement au repli sur soi dès lors que le souci connaîtrait la satisfaction, repli qui ne ferait qu'un avec l'oubli. L'animal aussi manifeste et en ce sens exprime la faim, la peur et la satisfaction. Mais la différence est sensible entre la satisfaction qui suit le fait de ne plus avoir peur ou faim, et celle qui vient de ce que l'on a été compris en tant qu'éprouvant la peur ou la faim.

Le contentement de l'homme est inséparable de la compréhension par l'autre. La possibilité du langage, comme Descartes le faisait déjà remarquer, ne se trouve pas seulement dans la possession d'organes ou la disposition d'un mécanisme qui en contiendrait virtuellement l'usage, elle ne se trouve pas réduite non plus, faudrait-il ajouter, dans l'existence d'une langue transmise et transmissible, mais au moins autant dans la nécessité pour l'homme de passer par l'autre homme pour parvenir à soi. Le recours à la parole témoigne en ce sens d'une incomplétude ou d'une imperfection de l'homme, qui parle d'abord parce qu'il a quelque chose à dire. Nous sommes spontanément hors de nousmêmes, et l'expression consciente ou volontaire, l'interpellation de l'autre, ne seraient pas si impérieuses et « vitales » chez l'homme si chaque individu n'avait pas le sentiment confus que c'est par ce biais, ou par cette médiation, qu'il fallait passer pour avoir une prise sur les choses. On rejoint ici l'idée maintes fois avancée d'un inachèvement et de la prématuration de l'être humain. On ne remarque pas suffisamment à quel point l'homme est mal adapté à la nature, et comme s'explique, par là, la double et inséparable invention du langage et de la technique, du « geste et de la parole ».

Mais l'extériorisation du corps et de la pensée ne constituerait pas l'accès obligé à l'humanité si l'homme n'était pas lié à l'autre homme d'une façon toute différente de celle qui lie un animal à ses congénères. Aristote a vu le premier que l'homme était de tous les animaux le plus imitateur. De tous les facteurs qui contribuent à l'acquisition de la pensée conceptuelle, l'imitation est, pour Konrad Lorenz, celui pour lequel l'homme manifeste une compétence toute particulière ${ }^{3}$. Le perroquet et le singe sont devenus des

\footnotetext{
${ }^{3}$ Lorenz, 1975, pp. 208-209. Tinbergen (1967,p. 100) écrit aussi : « l'imitation réelle est extrêmement rare chez les animaux ». Cf. également, Tinbergen, 1980, p. 201.
} 
symboles dégradants de l'imitation, alors que l'homme les laisse loin derrière lui dans la perfection d'une telle disposition. L'homme doit singer l'homme pour être un homme, il doit répéter comme un perroquet ce qu'il entend pour le dire à son tour en en comprenant le sens. L'imitation précède la compréhension, comme la génuflexion amène, selon Pascal, la foi. À force d'être un autre, l'homme finit par être lui-même.

C'est donc "la passion d'être un homme », selon Lacan, qui fait l'homme. Autant dire que l'homme n'est au fond jamais vraiment sûr d'être un homme. Pourquoi chercherait-il avec une telle obstination «la différence entre l'homme et l'animal», sinon pour donner au trouble sur son identité une issue objective et rassurante ? Or il est tout de même étrange que tant de différences, toutes plus spécifiques les une que les autres, ne parviennent pourtant pas à l'assurer de son identité et à lui faire savoir ce que c'est qu'être un homme. Il ne lui suffit pas de se distinguer par le volume de son cerveau, la station droite de son corps, la possession d'un langage symbolique, ou par sa légendaire sociabilité ; il ne voit là que manifestations diverses d'une essence qui ne se donne pas elle-même. Rien ne contraste davantage avec ce doute sur l'humain que la tranquille identité à soi de l'animal. Un chimpanzé n'est pas un chimpanzé, a-t-on pu dire (Yerkes), pas plus qu'un rat n'est un rat ${ }^{4}$. Mais si l'on peut appeler un chat un chat, à quoi cela nous avance-t-il d'appeler un homme un homme ? J'ai toujours été intrigué par cette remarque de Locke au sujet des noms propres et des animaux : "je suis persuadé que si nous étions obligés de faire mention des chevaux particuliers aussi souvent que nous avons l'occasion de parler de différents hommes en particulier, nous aurions pour distinguer les chevaux des noms propres, qui nous seraient aussi familiers que ceux dont nous nous servons pour désigner les hommes, que le mot de Bucéphale, par exemple, serait d'un usage aussi commun que celui d'Alexandre. Aussi voyons-nous que les maquignons donnent des noms propres à leurs chevaux aussi communément qu'à leurs valets, pour pouvoir les connaître, et les distinguer les uns des autres $»^{5} \ldots$

\section{L’homme, « spécialiste de la non spécialisation »}

Il me semble que cette observation enseigne surtout que l'homme ne peut avoir de relation avec ce qui l'entoure qu'en lui donnant un nom, et que l'individualité des choses comme des êtres est constituée par leurs noms, bien davantage que par leurs formes ou figures. Mais du fait même de nommer les choses ou les êtres, on les distingue de leurs classes ou de leurs espèces. Les choses ou les êtres à qui l'on donne des noms propres acquièrent par là une sorte d'individualité et un semblant d'humanité. L'expérience nous montre suffisamment qu'une relation d'intimité avec une chose ou un être sollicite une appropriation par un nom. Le chat ou le chien «domestiques » deviennent en quelque sorte des «personnes » quand ils acquièrent une identité par le nom, dès qu'on entre dans la sphère de l'intimité et du lien personnel, il n'est plus possible d'appeler un chat, un chat. L'identité personnelle sépare l'individu de son espèce. Si nous ne sommes pas sûrs de savoir ce que c'est qu'être homme, c'est sans doute parce qu'on ne peut l'être sans être un individu distinct et séparé de tous les autres, et par conséquent qu'en cessant d'être homme - au sens d'une généralité d'espèce. L'animal, par ce que nous en savons, n'est lui-même qu'au sein de son espèce (c'est ce que souligne la phrase de Yerkes citée

\footnotetext{
${ }^{4}$ Voir le si troublant chapitre X de L'Agression, 1969.

${ }^{5}$ Locke, 1972, p. 329 ; voir aussi Bergson, 1970, p. 1130.
} 
plus haut). S'il y a conscience, elle ne peut être à ce niveau qu'une conscience d'appartenance à une " famille », un groupe ou une race, alors que la conscience chez l'homme correspond à ce moment de séparation d'un individu avec tous les autres qui le rend sujet ou ego.

L'animal hérite d'une identité qu'il revêt aussitôt qu'il naît, l'homme constitue la sienne propre dans une relation à autrui qui lui apprend tout, et surtout le sentiment d'être soi, la subjectivité même. Si le fondement de celle-ci est, comme l'a dit Benvéniste, dans l'exercice de la langue (1966, p. 262), la position égale et symétrique des consciences qui se marque par la substitution du toi au je est la véritable condition de l'accès à l'humain. Élection, et non sélection. C'est pourquoi la possibilité qu'a l'homme de rendre humain ce qui l'entoure, et ce avec quoi il vit, est quasiment illimitée et engendre l'inévitable péché d'anthropomorphisme, car les objets inanimés ont une âme sitôt qu'ils ont un nom. La lutte des consciences conserve bien sûr toute la vérité que Hegel y a décelée, à ceci près qu'elle caractérise aussi les nombreuses situations de rivalité pour le prestige, telles que les décrivent les éthologues. Là non plus il n'y a pas de lutte réelle, mais un comportement tout aussi symbolique pour des enjeux qui ne le sont pas moins. On a voulu voir dans cette lutte pour la reconnaissance la marque spécifiquement humaine du désir, mais à tort croyons-nous, et il se pourrait bien que les conduites symboliques liées à l'agression et plus généralement à la hiérarchie rendent plutôt perceptibles la continuité des sociétés humaines et animales. Nous suivons donc M. Serres (1985, p. 75) quand il écrit : « ...la dialectique décrit la logique des anthropoïdes. L'homme naît quand il en voit la fausseté $\rangle^{6}$. L'identification par le biais des circuits symboliques dont sont empreints les comportements sociaux nous semble donc ce par quoi la société animale perdure dans 1' « humaine ». Car il y a aussi des façons tout à fait humaines d'être animal, et pas seulement la «bestialité ». Quand les hommes se situent les uns par rapport aux autres, quand ils se jaugent et évaluent par signes leurs biens, leur puissance ou leur place, ce sont là des modalités typiquement humaines de fonctions dont on trouverait aisément des expressions différentes dans d'autres organisations sociales. La couleur de la cravate possède une valeur de reconnaissance et d'identification sociales comparable à la tache du rouge-gorge dans sa société (Leroi-Gourhan, 1965, p. 35).

L'idée même de comportement social dit assez l'anonymat des termes qui servent de supports aux rapports symboliques et qui ne servent qu'à manifester la relation fonctionnelle des êtres entre eux, dans une société où la communication se fait à distance et par code. Si l'homme ne parle vraiment que lorsqu'il parle à quelqu'un, alors n'est-ce pas du code de signaux plutôt que du langage que relèvent la plupart des messages induits par le comportement? Et n'est-ce pas aussi pour cette raison que les activités humaines se laissent si bien décrire en termes de comportement? Il ne nous vient jamais à l'esprit d'évoquer en ces termes ce que fait ou dit une personne de notre entourage. Mais l'idée de comportement s'insinue en revanche dès que l'on a conscience d'une bizarrerie ou d'une anomalie qui rend quelque peu étrangère à la compréhension que nous en avons ordinairement la personne chez qui on l'observe. Le comportement rend distant du regard l'être qu'on met ainsi en observation. On recourt à l'idée du comportement quand l'inhabituel se glisse dans la compréhension ordinaire que nous avons d'un être. Le fait même

\footnotetext{
${ }^{6} C f$. également, Tinbergen, 1980, p. 247.
} 
de décrire ou de définir un comportement normal manifeste le soupçon d'anormalité qui motive une telle démarche.

L'étude des animaux et des hommes sous l'angle du comportement n'exercerait pas une telle fascination si elle ne rendait pas, par le choix même d'une telle perception, étranger ce qui nous semble proche et familier ${ }^{7}$. Le comportement est ce par quoi le sujet glisse du côté de l'objet et s'éloigne par là même de la compréhension intime que j'en ai. C'est alors son corps qui parle. Mais on ne comprend pas un corps, on le déchiffre, on tente de surprendre une signification qui s'esquisse. En ce sens, parler, c'est échapper à son corps, le mettre à l'arrière-plan de la scène que l'ego occupe avec un autre, plus que devant lui. Réciproquement, je ne peux écouter ce que me dit quelqu'un et en percevoir objectivement le corps : plus tard je me souviendrai des paroles entendues, mais il me faudra faire un effort pour retrouver tel trait du visage ou tel aspect du corps.

La tentation d'humaniser les animaux et d' " animaliser» les hommes ne serait pas un procédé courant de la fiction si la réalité n'y prédisposait pas, en ne nous donnant de l'existence des autres hommes d'autres «preuves » que leur corps. Descartes se demandait s'il ne pourrait pas se faire que les chapeaux et les manteaux qu'il percevait de sa fenêtre puissent recouvrir des spectres ou des automates. Mais en admettant qu'il s'agisse de "vrais hommes », qu'est-ce que cela change ? N'aurais-je pas toujours affaire à des corps qui ne me livrent que «médiatement» une signification comportementale ? Je vois des gens courir pour attraper l'autobus ou se frotter les mains parce qu'ils ont froid. J'assemble la signification par «morceaux » même si j'aperçois synthétiquement la situation. Rien de tel ne se passe quand quelqu'un me parle : c'est lui que je vois et non son corps et ses motivations. Le langage fait véritablement sortir l'ego de son corps et l'érige en sujet devant celui qui le comprend. Il ouvre une brèche dans la muraille de l'intimité, corporelle ou sociale. Lorsque je reconnais des hommes dans la rue, ma perception atteint un type ou une espèce, une généralité comparable à celle d'autres espèces. L'identité d'individus ainsi appréhendés est une identité spécifique : c'est la propriété commune aux membres d'une classe, terme commun à la zoologie et à la sociologie.

Il y a comportement humain là où une succession d'actes ou d'attitudes semble régie par une loi qui transcende les individualités et leur confère l'unité d'un type ou d'une espèce. C'est comme si les individus étaient les acteurs de rôles auxquels leurs interprétations ne pouvaient apporter que de minimes variations. Qu'il y ait alors une corrélation entre l'empreinte de l'espèce et le degré de spécialisation n'est pas très surprenant car l'uniformité ne va jamais sans une étroitesse fonctionnelle. Plus on «descend» dans l'échelle des espèces animales, moins on trouve d'écarts individuels par rapport au type (Schopenhauer, 2004, p. 176), et plus parfaite est l'exécution de la tâche indéfiniment accomplie. Lorenz appelle l'homme le spécialiste de la non-spécialisation (1974, pp. 211-223), mais nul mieux que lui n'a mis en évidence la continuité des sociétés animales et humaines au niveau des comportements symboliques et des processus de ritualisation.

Lorsque Lorenz décrit le cérémonial de triomphe des grues, il ne peut se retenir de leur prêter les paroles toutes faites qu'en des situations analogues les hommes s'échangent (Lorenz, 1969, p. 69 et pp. 172 sq.). Son lecteur, quant à lui, éprouve le sentiment

\footnotetext{
${ }^{7}$ Cf. Morin, 1974, le chapitre V et les communications de M. Chance et de H. Montagner.
} 
mélangé de comique et de malaise qui apparaît généralement quand on se voit jouer un rôle et exécuter machinalement des propos et des gestes que, dans le feu de l'action, nous pensons être spontanément nôtres, tant il est vrai que «...nous sommes automates dans les trois-quarts de nos actions » (Leibniz). Si nous tentons d'analyser ce sentiment trouble, nous y découvrons sans doute un effet de la répétition et d'un mécanisme qui tourne à vide ${ }^{8}$. Cela explique le comique qu'engendre inévitablement le sérieux ou le sériel, non le malaise éprouvé devant le spectacle de l'animal ou de l'homme dont l'existence tourne en rond dans les circuits symboliques montés dans leurs espèces respectives. C'est que la conscience d'une extrême légalité enfouie dans l'individualité même ne va pas sans le soupçon de l'anormalité . Husserl dit que «les animaux sont essentiellement constitués pour moi comme les "variantes" anormales de mon humanité » (1969, p. 107). Le trouble ressenti devant l'animal qu'un mur de sérieux sépare de lui-même et des autres a quelque chose de commun avec l'impatience qui nous gagne devant un interminable commencement, un départ sans cesse différé, un geste dont l'esquisse se développe indéfiniment. Rien n'est sans doute plus symbolique et formaliste qu'un animal pour qui le réel ne semble être que le prétexte ou l'occasion de faire jouer avec virtuosité des mécanismes qui fonctionneraient aussi bien sans cette « chiquenaude », comparable en cela au paranoïaque qui ne rencontre la réalité que sur le mode de la confirmation. Si le leurre marche si bien avec les animaux, c'est sans doute parce que leur " problème » n'est pas de savoir s'ils se trompent, mais plutôt de montrer qu'on ne les trompe pas. Comme la sentinelle dont le sens de la discipline et de sa mission prévaut sur l'usage du jugement, et qui tire sur tout ce qui bouge.

« L'animal est sûr de lui-même... », a dit Bergson (1970, p. 1092). Une telle « sûreté » est inversement proportionnelle à la conscience de soi ; elle n'est jamais plus forte ni plus entière que là où le sujet ne se voit plus agir, comme dans le somnambulisme et dans la plupart des actions que nous faisons sans y penser. Il faut pourtant la distinguer de l'assurance, acquise par essais, tâtonnements et rectification, au terme d'un long processus d'hominisation. L'animal donne moins l'impression de grandir que de se déplier et de parvenir très rapidement au plein emploi de ses capacités. Le terme de « sûreté » exprime cette maîtrise sans travail que l'on reconnaît, chez les êtres qui en sont doués, à l'absence de toute hésitation, à ce qu'elle ne nécessite pas, ou fort peu, d'appréhension par imitation, ni la prise en compte d'un autre point de vue sur la situation.

On comprend alors pourquoi Machiavel demandait au prince de ne pas s'encombrer du préjugé de l'humain et d'imiter plutôt le lion et le renard pour acquérir (ou développer) la sûreté commune à la force de l'un et à la ruse de l'autre. Mais le principal conseil du «docteur des Princes» (« ...il est beaucoup plus sûr de se faire craindre qu'aimer... ») indique que la véritable vertu, celle qui tranche avec le bavardage moralisateur, consiste pour un homme politique à avoir l'audace de devenir un animal politique, à l'exemple d'Hannibal et «...de son inhumaine cruauté, laquelle va ensemble avec ses

\footnotetext{
${ }^{8} C f$. la notion de « réaction à vide » chez Lorenz (1974, p. 28) ; Tinbergen (1980, p. 151) dit que «...les psychologues [...] se rendent rarement compte de ce que, à strictement parler, ce n'est pas ses petits ou sa nourriture que l'animal s'efforce d'atteindre, mais l'accomplissement même des activités maternelles ou nutritionnelles ».

${ }^{9}$ C'est ce que remarque très justement Canguilhem (1971, p. 168) : «Qui voudrait soutenir, en matière de psychisme humain. que l'anormal n'y obéit pas à des normes ? Il n'est anormal que parce qu'il leur obéit trop ».
} 
infinies vertus... » (Machiavel, 1952, pp. 339-340). Machiavel, avec sa volonté de vérité effective, n'exhorterait pas un homme à se faire animal et inhumain si, dans son esprit, l'humanité ne désignait pas qu'un préjugé ou une notion morale. Pour s'en délivrer, il suffit de ne pas avoir à passer par le point de vue de l'autre pour constituer le sien.

La sûreté ne peut être le fait d'une conscience qui se sait irréductible à toute autre, et qui par conséquent est scindée par la dualité du moi et de l'autre - d'une conscience affectée par le sentiment de l'identité personnelle. L'animal au contraire (mais comment le savoir ?) doit plutôt tenir son identité du sentiment de l'appartenance à une espèce ou à un groupe. Il est probable qu'un animal reconnaisse dans l'autre avant tout, et peutêtre uniquement, un congénère. C'est l'espèce qui soude ici « les liens personnels » et qui régule l'amitié, l'amour ou l'agression. On peut trouver quelque chose d'analogue dans « les sociétés closes » où les individus doivent au sentiment d'appartenance, et à l'incorporation profonde des rites et symboles, la conscience d'être " membre », et non la conscience d'être soi, indépendamment de toute inscription territoriale. À ce niveau une famille de rats ou une tribu de singes sont-elles fondamentalement distinctes d'une communauté de membres soudés par le sentiment nécessairement trans-individuel de l'appartenance ? On y trouve là ces vertus dont on déplore quelquefois le défaut chez les hommes : la solidarité des membres, le respect sacré de la hiérarchie, des rites et des traditions invariables, et... la férocité sans limite envers l'étranger, l'autre, justement.

\section{Être ou penser?}

Il faut une éducation et un progrès de la réflexion pour reconnaître dans l'homme un alter ego ; les barrières communautaires donnent aux individus d'abord un sentiment plus immédiat d'identité sous la forme de l'appartenance. Sentiment que les individus surmontent peut-être, en accédant à la conscience de leur identité personnelle et de son universalité. II est douteux que les animaux franchissent jamais les barrières de leurs espèces et les limites de leurs territoires, qu'il y ait chez eux découverte de leur commune animalité. Il n'y a de «monde animal» que pour l'homme, à qui la science et la philosophie inculquent le sens de l'animal. Sans cette sortie de soi et de chez soi, l'individu ne perçoit que ce qui est perceptible, les différences visibles, sensibles entre les « races » d'homme, et les ressemblances entre membres d'une même race. L'homme, comme horizon de l'universalité, n'est visible qu'aux yeux de l'âme, d'une pensée qui reconnaît le fait du langage dans la variété des langues.

Aucune observation empirique ne rencontre quelque chose comme l'homme ou l'animal. Seule une idée de la raison peut se donner l'homme comme exigence infinie et idéal régulateur. L'homme n'est jamais sûr d'être un homme. L'insécurité de l'essence d'homme est aussi ce qui oblige l'homme à déployer en tous sens son activité, à avoir le sens du possible et à échapper aux spécialisations qui cantonnent les espèces, les groupes, les professions dans un territoire symboliquement défini et à un langage codé ou administratif. L'homme qui se prend pour un homme tourne indéfiniment sur le circuit symbolique de son identité imaginaire, comme «le garçon de café » de Sartre. La perfection de son geste et la reproduction de son code lui masquent l'insécurité et l'imperfection chronique de son essence. Le leurre suprême, pour l'homme, est de se croire homme, par droit divin. C'est l'homme à qui son essence est un dû, c'est l'homme sûr de lui-même par groupe, « corps » ou ethnie interposés. 
II me semble que l'humain ne peut consister que dans le doute sur l'humain, dans ce trouble qui saisit l'ego au bord de ces trous d'animalité que sont les automatismes corporels et sociaux. Mais en reprenant une réflexion dont le souffle est quelquefois coupé par le regard porté vers l'abîme de l'animalité, l'ego ne trouvera pas l'assurance qu'il cherche en affirmant qu'il est un homme. Cette croyance peut le rassurer, elle ne saurait l'assurer de ce qu'il est. Jamais le face à face de l'homme comme espèce avec l'animal ne pourra dénouer la question de son essence. Je ne vois pas de raisons autres qu'idéologiques pour lesquelles il faudrait s'assurer de l'existence d'un passé ou d'un saut dans l'évolution du vivant, et multiplier indéfiniment les «Rubicon » dont on dit de chacun d'eux qu'il est décisif. L'homme en tant qu'espèce peut bien, comme chaque espèce, présenter des caractéristiques uniques, mais il n'y a aucune raison pour qu'une science de l'homme soit, elle, d'une autre espèce que celle qui s'applique à connaître les caractères et les comportements des animaux. L'anthropologie en ce sens ne serait qu'une annexe de la zoologie.

Mais connaître l'homme n'implique nullement de savoir ce que c'est qu'être un individu identique à soi-même, ou ce que c'est qu'être moi. Ces deux plans sont tout à fait distincts, comme Descartes l'avait bien vu. Son traité de l'Homme ne dit pas un mot sur l'humain. C'est à se demander s'il n'y a pas quelque ironie dans ce titre, suivi d'une description de la machine du corps humain. Même là où l'on attendrait que Descartes nous dise quelque chose de l'humain, dans les Passions de l'âme (et non de l'homme), on se trouve une nouvelle fois devant une stricte distinction entre une description et une explication mécanistes des passions (qui n'est pas sans analogie avec l'étude de Darwin sur les émotions), et une morale que l'âme se donne pour faire de ces passions un bon usage. Cherchons donc dans les Méditations. Il y est beaucoup question de Dieu, de l'âme, de l'esprit, de l'entendement, de la nature des corps physiques et de celle du corps humain - mais d'homme, point ou presque pas. La métaphysique, avant d'être la recherche d'un fondement pour la science, décrit la progression d'un esprit d'abord et spontanément confiant (envers le témoignage des sens et le témoignage d'autrui) du doute sur ce qu'il sait et ce qu'il est jusqu'à l'acquisition d'une assurance rendue possible par l'élucidation du problème de l'erreur et la dissipation du soupçon d'être trompé. La Méditation première rappelle l'état de confiance totale et passée, dont on peut dire en généralisant qu'il constitue le véritable environnement de l'homme naissant ainsi que la condition de tout apprentissage. Mais le réveil difficile de l'esclave qui jouit dans son rêve d'une « liberté imaginaire » est encore retardé par des expressions spontanées d'une confiance (ou d'une croyance, c'est égal) finissante. Ainsi cette exclamation de Descartes, après la célèbre évocation fugitive de la folie : «Toutefois j'ai ici à considérer que je suis homme ». J'y vois pour ma part beaucoup moins la manifestation de quelque chose «d'antérieur ou d'extérieur » au discours cartésien que la tentation, qui va vite se révéler vaine, de se raccrocher à une certitude ancienne qui commence seulement à se morceler. C'est le reste d'un savoir ancien, vieux comme est toujours vieille la confiance, et que le fait de son ancienneté suffit, pour la conscience qui s'éveille, à rejeter du côté du non-savoir.

A-t-on suffisamment remarqué que l'avancée méthodique jusqu'à la découverte du cogito a comme premier résultat la suspension de la validité de l'idée d'homme ? Que le cogito qui donne en un éclair au moi, qui s'en inquiète depuis le début de la Méditation seconde, l'expression simple et entière de son identité, que le cogito, donc, oblige l'esprit 
à mettre entre parenthèses l'idée d'homme dont Descartes s'aperçoit qu'elle est complexe et incertaine ? Comment ne pas rapprocher la mise en garde que Descartes se fait à luimême "...de ne prendre imprudemment quelque autre chose pour moi...", de cet aveu de croyance (ou de confiance) qui résume en un trait tout le passé pré-philosophique de l'homme :

«Qu'est-ce donc que j'ai cru être ci-devant ? Sans difficulté, j'ai pensé que j'étais un homme. Mais qu'est-ce qu'un homme? Dirai-je que c'est un animal raisonnable ? Non certes : car il faudrait par après rechercher ce que c'est qu'animal, et ce que c'est que raisonnable, et ainsi d'une seule question nous tomberions insensiblement en une infinité d'autres plus difficiles et embarrassées, et je ne voudrais pas abuser du peu de temps et de loisir qui me reste, en l'employant à démêler de semblables subtilités »(Descartes, 1964-1974, t. 9, p. 20).

Regardons dans la suite des Méditations: Descartes n'apporte jamais de réponse à cette « question »-dont Foucault a montré qu'elle était le point d'Archimède de l'épistémè moderne -, trop endurcie dans « son sommeil anthropologique » pour soupçonner cette identité imaginaire de n'être « qu'un songe $»^{10}$. Inversement, il monte un dispositif imaginaire, il institue une expérience de pensée pour déduire une différence qui est moins celle de l'homme et de l'animal que celle de la pensée et de la machine. Ce ne sont pas des êtres et moins encore des modes d'être que Descartes oppose ou compare lorsqu'il traite de la question de la différence homme/animal, ce sont des principes distincts de fonctionnement, des modes d'organisation, qu'il cherche à dégager en se tenant au plus près de ce qui est observable, et seulement observable, dans des comportements qui nous sont trop proches, trop immédiatement compréhensibles, pour que nous puissions mettre en question l'analogie ou la parenté présumée de l'homme et de l'animal. Cette question est donc de nature épistémologique, et c'est dans les limites d'une enquête de ce type qu'entend se tenir Descartes dans tous les textes où il en parle. C'est seulement par la suite, chez ses «disciples » (sic !), qu'elle va dériver dans les eaux agitées des enjeux théologiques. Tentons d'établir ce que dit effectivement Descartes sur ce sujet.

\section{L'animal comme machine}

Il y a deux façons d'aborder cette question. Dans la perspective de l'histoire des sciences, pour mesurer l'influence de la thèse cartésienne sur les savants et les philosophes du XVII ${ }^{\mathrm{e}}$ et du XVIII ${ }^{\mathrm{e}}$ siècles (jusqu'à La Mettrie et le passage ou le saut de l'animal à l'homme machine); ou bien en la rapportant au cartésianisme, non pas comme une partie du système (dans lequel elle n'occupe d'ailleurs pas une place précise), mais dans la perspective du développement de la pensée cartésienne. Or celle-ci, comme toute véritable philosophie, détermine, diversement mais avec constance, une façon pour l'homme de se rapporter à lui-même et de parvenir à la connaissance de soi, en surmontant ce qui y fait obstacle: préjugés issus de l'enfance, vaines imaginations, fausses croyances. La question des animaux-machines est d'abord un parfait exemple du changement d'attitude que doivent produire dans l'esprit des hommes la science et la philosophie lorsqu'elles parviennent à établir une liaison simple et intelligible entre des phénomènes particuliers et des causes communes aux choses naturelles. La résolution

\footnotetext{
${ }^{10}$ Henry (1985, voir notamment le chapitre III) a très bien montré l'exclusion de la problématique de l'homme par le cogito ainsi que le contresens sur lequel repose le procès que Heidegger fait au cartésianisme.
} 
de cette question doit permettre aux hommes de se dégager des préjugés qui les accompagnent depuis leur enfance et, du même coup, de mieux se connaître eux-mêmes. C'est un moyen indirect qui est ainsi donné aux hommes de connaître, ou mieux de reconnaître, leur nature propre, par différence avec la nature animale en général. La connaissance de l'animal ne mène à celle de l'homme que par la différence qu'elle met en évidence entre la conduite machinale et le comportement intelligent. Descartes distingue deux types d'actions fondamentalement différentes entre lesquelles il y a bien solution de continuité, il n'établit pas une hiérarchie entre des niveaux de complexité afférents à des classes d'êtres vivants distincts. On ne s'élève pas de l'animal à l'homme, on ne distingue pas l'homme par une propriété qui lui serait spécifique dans le genre qu'il partage avec les autres animaux. N'est-ce pas ce mouvement d'esquive qu'exprime la question (formulée en des termes très proches de ceux de Montaigne) que Descartes se pose à lui-même dans le cours de la Deuxième méditation?

Cette mise entre parenthèses de la signification immédiate du terme d'animal n'est sûrement pas sans arrière-pensée, elle permet de considérer la signification du terme d'homme en elle-même, sans référence à la communauté formée par les êtres vivants. L'homme n'est pas compris dans le genre animal ; il a un sens propre, plus clair que celui d'animal raisonnable. En toute rigueur, le cartésianisme ne peut pas recevoir une telle expression, car l'animal est privé de raison (Descartes pense pouvoir montrer que rien dans les comportements des animaux ne témoigne de la présence de la raison en eux), de cette raison dont la possession fait de l'homme un être pensant, et non un être vivant. Il y a bien deux principes, irréductibles, auxquels se rapportent les actions des hommes : un principe qui gouverne les fonctions corporelles stricto sensu et qui leur est commun avec les animaux, un deuxième principe, tout à fait différent du premier, qui rend compte de tout ce qui se fait par la pensée, de tout ce qui l'implique, si peu que ce soit.

Cette dualité de principe ne redouble pas simplement la dualité ontologique de l'âme et du corps, car toutes les actions (et les passions) de l'homme en tant qu'homme sont de l'ordre de la pensée ; seules celles qui se font sans l'entremise de la pensée devant être tenues pour seulement corporelles, c'est-à-dire machinales ou automatiques. Ainsi, les fonctions qui s'accomplissent sans le concours de l'esprit sont-elles bien mieux effectuées que si l'esprit s'en mêlait. Une horloge montre mieux les heures que ne le feraient les hommes avec toute leur science et toute leur prudence ${ }^{11}$.

Cette sorte de naturalisation de la machine permet alors de comparer et de faire communiquer les produits de la nature et ceux de l'art, de «lever la barrière » (ce qui ne veut pas dire « d'effacer les frontières ») entre le vivant et le mécanique, et de poser le rapport homme / animal en termes d'esprit et de corps, c'est-à-dire de tout ou rien, et non de plus et de moins. Ce n'est pas que Descartes n'ait pas de considération pour les différences et les singularités qui font la matière de la réflexion de Montaigne par exemple, mais il pose la question autrement en interposant la machine entre l'homme et l'animal. La confrontation entre l'homme et l'animal (si jamais confrontation il y a) ne se fait pas directement, ni de l'intérieur (l'homme cherchant à deviner ce qui se passe dans la tête de l'animal, ou à y retrouver des sentiments ou des pensées semblables aux

\footnotetext{
${ }^{11}$ Cf. Lettre à Newcastle du 23 novembre 1646, AT IV, p. 575 : « elles [les bêtes] agissent naturellement et par ressorts, ainsi qu'une horloge, laquelle montre bien mieux l'heure qu'il est que notre jugement ne nous l'enseigne ».
} 
siens), mais par le biais de la machine dont la vertu est d'extérioriser et d'objectiver un rapport qui resterait sans cela purement spéculaire. À chaque fois qu'il est question de comparer l'homme et l'animal, Descartes scinde en deux la question et confronte d'une part l'homme et la machine, d'autre part l'animal et la machine. Et sa méthode consiste seulement à se demander jusqu'où peut aller cette confrontation, s'il y a un point où une différence apparaît, ou s'il n'y en a pas, ce qui, en ce cas, ne permet pas d'éliminer l'hypothèse de l'automatisme ou la pertinence de la comparaison avec la machine. La thèse (si ce terme convient) de Descartes n'est pas que les bêtes ne pensent pas, ni même que ce sont des machines, mais qu'il n'y a aucun argument, aucune raison, aucune preuve qui permette de dire qu'elles pensent et ne sont pas que des machines ${ }^{12}$. C'est une thèse principalement dirigée contre ceux qui assurent (mais sans le prouver) que les bêtes pensent et agissent comme nous, et même quelquefois mieux.

Ce qu'il faut alors se demander, c'est pourquoi Descartes multiplie les assauts contre ce préjugé sans doute mieux encore partagé que le bon sens, car ce ne sont pas les animaux en tant que tels qui préoccupent Descartes. Ce qui se joue à travers la question des animaux machines est tout autre que la question du sort de l'animal, ou même que la question de la différence entre l'homme et l'animal. La vraie question est celle des limites, et donc aussi de la puissance, de l'automatisme corporel, c'est celle de l'analogie, fonctionnelle et non pas sensible, du corps et de la machine (l'horloge). Toute la philosophie de Descartes, et cela dès le début, peut être considérée comme une critique de la ressemblance et des préjugés qui en dépendent. La physique commence par surmonter cet obstacle en cherchant des équivalents mécaniques des phénomènes naturels, comme la lumière. Il ne faut pas penser que les choses matérielles, les corps physiques sont comme nous les voyons et sentons. Descartes prolonge cette critique dans le domaine du vivant, il demande alors au lecteur de ne pas croire qu'il doit y avoir dans les corps vivants, " animés », une ou plusieurs âmes qui les dirigent, ni que l'on doive conclure de la ressemblance entre certaines actions des animaux et de l'homme à l'existence dans leurs corps des pensées ou sentiments liés pour nous à ces actions ou mouvements corporels. Les mouvements corporels qui sont, pour certains d'entre eux en tout cas, accompagnés de pensée n'ont pas pour autant la pensée pour cause, ils peuvent être, chez l'homme seulement, associés à la pensée, simultanés à la conscience que l'homme en a. Il est quasi inévitable que lorsqu'on voit des mouvements corporels ressemblants (comme ceux des passions : colère, peur, joie, tristesse, etc.) on imagine des sentiments semblables au dedans. Mais ces pensées analogiques ne sont peut-être dues qu'à l'ignorance de ce que peuvent les corps par leur nature propre, à la sous-estimation de l'art divin, toujours beaucoup plus admirable qu'on l'imagine, dont pourtant l'art humain des machines automates nous donne déjà quelque idée.

Comprise dans la perspective de la philosophie cartésienne, ladite théorie des animauxmachines porte témoignage de la puissance créatrice de Dieu, d'un Dieu constructeur et non d'une nature protectrice. Loin de conduire au rabaissement et au mépris de l'animal (on notera plutôt l'absence de tout propos normatif, de tout jugement de valeur sur les

\footnotetext{
${ }^{12}$ Cf. Lettre à Morus du 5 février 1649, AT V, pp. 275-279 : « J'ai tenu pour démontré que nous ne pouvions prouver en aucune manière qu'il y eût dans les animaux une âme qui pensât. [...] Cependant, quoique je regarde comme une chose démontrée qu'on ne saurait prouver qu'il y ait des pensées dans les bêtes, je ne crois pas qu'on puisse démontrer que le contraire ne soit pas, parce que l'esprit humain ne peut pénétrer dans leur cœur pour savoir ce qui s'y passe ».
} 
animaux), cette idée de la machine corporelle que Descartes substitue à l'idée traditionnelle de nature contraint l'imagination moyenne des hommes à reconnaître son incapacité à la représenter. C'est en n'imaginant pas l'animal mais en le concevant sur un plan qui n'est pas propice à l'imagination mais à la connaissance, c'est en déjouant le piège de la ressemblance que l'on peut se faire une idée de ce qu'il est, et de ce qu'il peut, par l'effet de sa propre nature. L'image de la machine refoule l'imaginaire de l'animal. L'idée de l'automatisme animal place donc la question sur un tout autre terrain que celui de l'intelligence ou de la bêtise des animaux (Descartes ne dit d'ailleurs pas que les animaux sont bêtes, ce n'est que pour les hommes qu'il parle de stupidité), sur le terrain, encore si peu foulé, de la connaissance scientifique et médicale du corps qui seule est à même de démonter la machine et d'expliquer pourquoi une machine est d'autant plus admirable qu'elle est toujours machine, qu'elle n'a besoin d'aucun esprit pour accomplir sa tâche et que cette autonomie par rapport à l'esprit la rend admirable. La question proprement cartésienne n'est pas celle de la différence de l'homme et de l'animal; ou plutôt, à travers cette question bien traditionnelle, trop peut-être pour la philosophie de Descartes, percent la nouveauté et la profondeur d'un questionnement méthodique sur les marques auxquelles on peut reconnaître l'action d'une machine et l'action de la pensée.

Le «test» imaginé par Descartes dans la $5^{\mathrm{e}}$ partie du Discours de la méthode procède, comme la fable du nouveau monde ou la supposition d'un malin génie, de la conscience de la nécessité de tordre le bâton dans l'autre sens pour convaincre les hommes malgré les préjugés qui imprègnent leurs croyances. La démarche de Descartes est celle d'un expérimentateur qui sait a priori ce qu'il faut prouver et qui part d'une hypothèse inhabituelle, voire invraisemblable, pour que le lecteur ne puisse s'appuyer sur des " évidences » communément partagées. La différence entre les hommes et les animaux est trop manifeste, rendue visible par trop de marques, pour qu'on puisse feindre de l'ignorer. Mieux vaut alors imaginer une expérience que personne n'a jamais pu faire et qui confronte non pas l'homme et l'animal, mais d'une part l'homme et la machine, d'autre part l'animal et la machine. Par cet artifice, Descartes ne permet pas à l'opinion de s'exprimer comme elle le fait ordinairement, c'est-à-dire bruyamment et abondamment, ajoutant sans fin une anecdote à une autre. Descartes sait bien que l'on ne peut apprendre quelque chose qui contredit nos préjugés qu'en recourant au jeu. Imaginons donc des machines aussi semblables que possible aux animaux que nous connaissons: comment reconnaîtrions-nous ceux-ci ? Ne sachant rien d'autre que ce que nous pouvons voir, nous ne pourrions pas distinguer le naturel de l'artificiel, ou le vivant de la machine, ces animaux-machines étant en quelque sorte du vivant plaqué sur du mécanique, pour paraphraser la célèbre formule de Bergson... On concéderait alors le caractère équivalent de ces machines animées et des animaux-machines.

Le même artifice appliqué aux hommes (et par ce mot Descartes ne désigne pas autre chose que les figures ou le style corporel des hommes) ne nous laisserait pas sans recours. Les deux moyens que nous aurions pour distinguer les vrais hommes des automates mettent en évidence la même chose : le caractère inimitable de ce qui procède de la pensée, aussi banale, aussi fruste qu'en soit l'expression. En effet, la caractéristique commune à la parole et aux actions humaines est à la fois l'extension indéfinie de leur champ d'application et la liberté de l'esprit dont elles témoignent. Les termes utilisés par Descartes pour souligner la spécificité de la parole par rapport aux imitations seulement phoniques des animaux sont : « composer un discours », « assembler des mots », termes qui supposent non seulement 
une activité de l'esprit dans le langage mais une initiative de celui qui parle, une invention même, car il n'est pas concevable qu'une machine soit programmée pour parler adéquatement en toutes sortes d'occasions ou de rencontres. Occasions ou rencontres parce que ce n'est pas une stimulation extérieure qui fait parler les hommes, mais une situation qui suscite, grâce à l'intelligence que l'homme en a, une réponse appropriée.

Ce caractère de plasticité et d'adaptabilité du langage se rencontre aussi dans les actions humaines dont chacune peut être moins parfaite que son analogue animale justement parce qu'elle n'est pas comme emboîtée dans une situation ou un contexte bien déterminés, mais traduit la polyvalence de l'intelligence qui peut s'appliquer à toutes les situations parce qu'elle ne dépend d'aucune d'entre elles en particulier et peut s'adapter à toutes : la raison, dit Descartes, est un instrument universel. Magnifique définition qui montre que le propre de l'homme, la raison, ne consiste pas dans une capacité particulière (parler, fabriquer, etc.) qui distinguerait l'espèce humaine des autres espèces, mais dans le pouvoir d'inventer ou d'instituer des signes pour se faire comprendre, dans la faculté d'agir intelligemment, c'est-à-dire de trouver la solution adaptée au problème rencontré, sorte de perception fine du moment ou de l'occasion qui se nommerait plutôt « jugement » qu' « intelligence » (au sens strictement et platement cognitif de ce terme). Plus cartésien que les cartésiens orthodoxes, Pascal fera écho à cette non-définition de l'homme en disant qu'il « ...n'est produit que pour l'infinité $»^{13}$.

La machine a donc surtout une fonction heuristique. En imaginant le corps comme une machine, Descartes se donne le moyen d'en observer l'intérieur comme s'il était transparent. Il n'a pas besoin de recourir à des choses ou êtres invisibles pour expliquer le visible. Il pose que tout est corporel dans le corps, que la différence est entre ce qui est trop petit pour être vu et ce qui est proportionné à notre vue. Une machine, parce que les organes, les membres, forment un assemblage, un tout si l'on veut. La machine peut être organique sans perdre son caractère de machine, elle peut même être appelée "vivante », si l'on y tient. Car, contrairement aux dogmatiques qui se réclameront du cartésianisme, Descartes se sert du mot «machine » en savant et expérimentateur, pour accroître la visibilité des choses et des êtres naturels et non pour déterminer quelque chose de leur nature. C'est pourquoi il peut dire qu'il n'ôte aux bêtes ni la vie, ni la sensibilité, ni l'âme à condition d'entendre ces mots de façon corporelle ${ }^{14}$. La machine de Descartes n'est pas insensible : elle peut agir, pâtir, sentir. Mais comme une machine, c'est-à-dire sans penser qu'elle agit, qu'elle pâtit ou qu'elle sent ${ }^{15}$. De ce mode de vie, il faut bien reconnaître que nous ne pouvons nous faire une idée distincte. Mais, justement, cette limite de la compréhension ne doit pas être franchie en posant une thèse négative comme « les bêtes ne pensent pas, donc elles ne vivent pas, ne sentent pas, pas plus que des horloges ou des instruments de musique ». Cette thèse, faut-il encore le dire, n'est pas celle de Descartes.

\footnotetext{
${ }_{13}^{13}$ Préface sur le Traité du vide, in Euvres complètes, 1963, p. 231.

${ }^{14}$ Cf. Lettre à Morus du 5 février 1649, AT V, p. 278 : « Je n'ôte la vie à aucun animal, ne la faisant consister que dans la seule chaleur du cœur. Je ne leur refuse pas même le sentiment [sensum] autant qu'il dépend des organes du corps ».

${ }^{15}$ Cf. Lettre à Plempius pour Fromondus du 3 octobre 1637, AT, I, p. 413 : « Il suppose que, selon moi, les bêtes voient de la même façon que nous, c'est-à-dire en sentant, ou en pensant qu'elles voient [hoc est sentiendo sive cogitando se videre] »; «Mon opinion n'est pas que les bêtes voient comme nous lorsque nous sentons que nous voyons [dum sentimus nos videre], mais seulement qu'elles voient comme nous lorsque notre esprit est appliqué ailleurs ».
} 


\section{L'homme : une chose qui doute}

La métaphysique cartésienne enseigne, comme jamais avant elle, ce que c'est que l'esprit, ou l'entendement, ou la raison, et qu'en cela consiste l'être ou l'identité du moi comme cette chose qui pense dans toute pensée d'autre chose, elle dévoile l'identité de l'idée de Dieu dans celle de l'infini; elle ouvre des perspectives pour la science de la nature du corps physique, du corps humain et animal, - elle n'a rien à dire de l'homme comme réalité sui generis. Descartes ne parle pas de l'homme, mais - et c'est tout différent - du composé, ou de l'esprit humain, ou de la nature de l'homme. L'homme prend toujours son sens de quelque autre chose qui le précède, l'éclaire ou l'explique : schématiquement, du moi (ego), de Dieu, de la nature. Et comme l'idée de moi-même est inséparable de l'idée de Dieu, « née et produite avec moi dès lors que j'ai été créé », il n'y a effectivement pas de place pour l'homme comme fait originaire entre l'animal et Dieu. L'union du fini avec l'infini - l'homme - n'est donc pas une idée simple ${ }^{16}$, mais complexe et constituée par des pôles qui ne fusionnent pas dans une quelconque " unité de l'homme ». C'est pourquoi l'homme est le seul être qui doute de son être, et qui ignore ce que c'est qu'être un homme, alors qu'il sait ce qu'il est lui-même (ego ipse), « une chose qui pense, c'est-à-dire une chose qui doute ». Une chose, notons-le bien, et ce terme est un de ces mots primitifs auxquels s'arrête le langage, et que toute définition obscurcirait. Entre l'altérité du corps (non ce corps « vécu », mais le corps mis en pièces par la pensée), et l'altérité de Dieu ou de l'idée d'infini, l'ego ne cesse d'aller et venir, à la recherche d'une identité spécifique (et non plus individuelle ou personnelle), d'un lieu où il trouverait la tranquillité de l'animal ou l'immobilité du divin - entre un être tellement fini qu'il semble n'avoir besoin de personne (anormalité de l'animalité) et un être infini qui ne dépend de rien d'autre que de lui-même (impénétrable mystère de la causa sui). Et pourtant, comme l'a dit un philosophe qu'on ne peut soupçonner d'humanisme, « il pourrait sembler que l'essence du divin nous soit plus proche que l'étrangeté des êtres vivants [...], que la parenté charnelle, abyssale, à peine pensable, avec l'animal ${ }^{17}$.

Peu de choses donnent autant que la vie animale le sentiment du destin. La « carrière » de chaque animal semble avoir été programmée de toute éternité, dans l'ordinateur de son espèce. Quand on voit les efforts prodigieux des psychologues des animaux pour arracher aux êtres les plus doués un semblant d'activité symbolique (et encore n'est-elle pas libre, mais obtenue à grands coups de procédures de renforcement), et quand on lit la passionnante et émouvante relation qu'A. Sullivan a faite du cas d'Helen Keller, enfant née sourde, muette et aveugle, qui découvre, invente presque, un langage à l'aide d'un alphabet digital, on ne peut s'empêcher d'opposer la vanité de tout effort pour faire sortir l'animal de lui-même à l'impossibilité pour l'homme de ne pas chercher, par tous les moyens, à sortir de soi. «Elle apprend parce qu'elle ne peut pas faire autrement » écrit, à propos de cette enfant, A. Sullivan ${ }^{18}$.

\footnotetext{
${ }^{16}$ Même si l'union de l'âme et du corps constitue une «notion primitive » qui n'est connue clairement que par les sens «en s'abstenant de méditer» (Lettre à Élisabeth du 28 juin 1643). Le fait de parler de l'homme en termes d'union d'âme et de corps, ou de composé, n'équivaut pas au fait de le désigner par le terme d'homme, qui serait en ce cas plus immédiatement intelligible que celui de composé, qui désigne, rappelons-le, l'interaction continue des deux substances, et donc l'expérience de leur « mélange ».

${ }^{17}$ Heidegger, cité par D. Franck, 1986, pp. 63-64.

${ }^{18}$ Cf. la relation qu'en fait Lorenz, 1975, pp. 243-255.
} 
Certes les animaux communiquent entre eux, mais comme on communique à l'intérieur d'une administration, par notes et instructions : pas de discussion, exécution ! Le dialogue entre le petit enfant et son entourage (dialogue pré-verbal, mais sans doute aussi pré-natal) manifeste ceci de remarquable que l'enfant fait sienne la pensée de l'autre en la percevant comme une question à lui poser. Les singes et les anthropoïdes, d'après Buytendijk, ne réalisent pas qu'une question leur est posée quand on leur demande d'exécuter une tâche (Buytendijk, 1965, p. 166). Au mieux, la question s'efface après que la réponse adéquate a été donnée ; elle ne continue pas de résonner dans l'esprit jusqu'à ce que le sujet en fasse un usage personnel et s'adresse à son tour à l'autre. De même, un enfant à qui l'on apprend quelque chose de nouveau n'éprouve pas de plaisir et de nécessité plus grands que celle d'éduquer son éducateur. Tout se passe comme s'il savait « d'instinct» que l'homme ne comprend qu'en faisant comprendre, et que la seule voie pour rendre vraiment sien un savoir reste de l'enseigner aux autres.

Mais, plus généralement, le monde n'aurait pas pour l'homme ce caractère indéfiniment et irréductiblement problématique si la structure foncièrement interrogative de la relation de l'un à l'autre ne reposait pas sur le fait que chaque sujet aperçoit dans un autre sujet la possibilité d'une expérience autre que la sienne, bien qu'installées l'une et l'autre dans un monde commun. La conscience est toujours conscience de la possibilité d'une autre conscience qu'elle-même, et par conséquent d'un autre point de vue. En termes leibniziens, l'esprit imite dans son département et son petit monde... ce que Dieu fait dans le grand, il n'est pas simplement comme les autres monades « miroir de l'univers » mais « image de la divinité » (Leibniz, 1972, p. 395). C'est pourquoi l'esprit construit un rapport entre lui et le monde, au lieu d'adhérer au monde comme à un milieu naturel. De là vient que la question de l'illusion (du rêve, de la folie, de la tromperie et de l'erreur) exige une certitude métaphysique qui puisse fonder, à défaut d'une théodicée ordonnée au principe du meilleur, du moins l'objectivité des perceptions claires et distinctes. Lorsque Descartes se demande pourquoi il juge que c'est la même cire qui demeure malgré ses incessantes altérations, il parle d'une « inspection de l'esprit », et d'un esprit humain en cela qu'il est capable de « distinguer la cire d'avec ses formes extérieures », tout de même que s'il lui avait «enlevé ses vêtements ». C'est précisément parce que l'esprit est capable d'apercevoir l'identité, qu'il distingue en toute chose un objet et en tout homme un sujet, quand bien même je ne verrais de ma fenêtre que « chapeaux et manteaux », ou une physionomie ou une couleur de peau qui, ainsi que des vêtements, dissimuleraient l'essence humaine de toute homme aux sens et à l'imagination. Aussi cette essence ne peut-elle être celle d'une espèce ou d'un genre, avec ses différences sensibles et caractéristiques, mais celle d'un ego et d'une âme par laquelle chacun est ce qu'il est, simple et peut-être première expression du principe d'identité. En paraphrasant une célèbre formule de Leibniz, on dirait qu'un homme, c'est un homme. Autrement dit, l'homme, parce qu'il désigne l'irréductible individualité de chacun, et n'est humain qu'ainsi, est une notion indiscernable. Mais l'impossibilité d'une définition de logique, par genre et différence, ne constitue que l'envers d'une extrême évidence : le fait d'être le même que l'autre en ce qu'il se constitue sur le mode indéclinable de l'ego. Bref un homme est une chose qui dit «je», chose sans forme ni figure extérieure, chose donc immatérielle ou métaphysique.

À travers ces multiples occurrences, une même idée circule, ou plutôt, c'est vers elle que convergent ces significations et d'autres encore. Être humain, c'est faire spontanément 
ce que l'on n'est pourtant pas obligé de faire. Plus encore, c'est s'abstenir quelquefois de faire ce que le règlement, la loi (des hommes ou celle de la nature) nous permettent ou nous obligent de faire. Un homme se montre « humain » en étant capable à certains moments de donner plus que ce qu'il doit, - d'enfreindre le règlement, les rites ou les usages symboliques de son groupe, de son clan, de son territoire et autres micro-espèces - de reconnaître l'homme nu. Les animaux ne sont pas vêtus et pourtant ils ne sont pas nus. Fourrures, plumages, écailles constituent autant de vêtements incorporés, marques signifiantes qui strient la surface visible et mélangent de façon inséparable l'essence et l'apparence.

Seul un être qui se sait lui-même au-delà des marques auxquelles le regard s'arrête peut avoir conscience qu'une essence se dissimule, invariante, dans le jeu sans profondeur des apparences. Le sens de l'humain serait alors le courage de « voir » la faiblesse et la vulnérabilité d'une essence foncièrement nue affleurer le plumage bariolé des apparences et des symboles - sorte de présence sensible de l'intelligible même. La rougeur, expression la plus éminemment humaine de l'émotion d'après Darwin (1981, p. 390), est la couleur de l'âme à fleur de peau. Les performances spécifiques de l'homme et le raffinement de la civilisation peuvent nous donner le sentiment de la puissance et d'une relative sécurité. Autre est celui d'humanité, qui ne va pas sans un certain désaccord avec les mécanismes naturels et sociaux et qu'expriment aussi bien le terme cartésien de «générosité » que celui, rousseauiste, de «pitié ». N'avons-nous pas quelque peu oublié ce que les philosophes classiques savaient : qu'être humain, c'est autant se rendre semblable à Dieu que semblable à tout ce qui souffre, être capable à la fois de se faire plus et moins qu'homme? 


\section{Chapitre 6}

\section{L'animal comme miroir de l'homme au $X V I^{e}{ }^{e}$ siècle}

JEAN-LUC GUICHET

L'objet de ce chapitre sera d'esquisser une clarification - inévitablement sommaire sur bien des points qui ne seront guère que mentionnés - de la situation du rapport de l'homme et de l'animal au XVIII ${ }^{\mathrm{e}}$ siècle. Le fait majeur caractérisant cette période est qu'elle apparaît comme une transition, un point d'équilibre fragile et provisoire entre, d'une part, des conceptions antérieures, métaphysiquement ou théologiquement plutôt assurées en règle générale de la différence ontologique des deux êtres et, de l'autre, des conceptions modernes, ou plutôt contemporaines, qui ne le sont plus du tout. Schématiquement, trois mouvements peuvent être repérés comme essentiels :

- au XVIII ${ }^{\mathrm{e}}$ siècle s'effectue un rapprochement décisif de l'homme et de l'animal, plus particulièrement au milieu du siècle ;

- ce rapprochement est tel que ce rapport de l'homme et de l'animal devient l'un des pivots essentiels de l'anthropologie moderne qui est en train d'émerger depuis le siècle précédent ;

- ce rapprochement induit une sorte d'inquiétude, de vertige, chez beaucoup, d'où des réticences, des réactions, et un mouvement d'éloignement après ce moment de rapprochement.

\section{L'animal et la nouvelle anthropologie}

Commençons par une citation, celle de la première phrase de la préface du Traité des animaux de Condillac publié en 1755 : «Il serait peu curieux de savoir ce que sont les bêtes, si ce n'était pas un moyen de connaître mieux ce que nous sommes » (2004, p. 111). Condillac s'inspire beaucoup ici d'une phrase de Buffon (1753) qu'il rappelle d'ailleurs tout de suite après : «S'il n'existait point d'animaux, dit M. de Buffon, la 
nature de l'homme serait encore plus incompréhensible ${ }^{1}$. Ainsi, cette idée - celle que l'animal constitue un terme de comparaison privilégié pour l'homme - est très partagée à l'époque. Or, si certes, l'animal a toujours plus ou moins tenu ce rôle - on peut notamment penser à Aristote -, cela était, nous semble-t-il, d'une tout autre manière. Par exemple, dans la perspective finaliste d'Aristote, la comparaison joue dans les deux sens : c'est tout autant l'homme qui, au titre du plus parfait des animaux, contribue à expliquer les animaux que l'inverse, et même davantage en droit, puisque, précisément, il est le plus parfait des animaux et qu'il présente donc de manière totalement actualisée ce que contiennent seulement potentiellement les autres êtres. L'animal constitue par conséquent un terme de comparaison, mais tout à fait infériorisé et la balance est très inégale. Plus tard, dans le monde chrétien, c'est évidemment Dieu la première source de lumière, toutes choses étant plus ou moins son miroir - Dieu se reflétant dans l'ensemble de la création - et particulièrement l'homme, ce que Pascal rappelle dans les Pensées d'une formule qui offre une symétrie frappante avec celle de Condillac et Buffon : privé de Dieu, l'homme serait « un monstre incompréhensible » à lui-même. ${ }^{2}$

$\mathrm{Au}$ XVIII ${ }^{\mathrm{e}}$ siècle s'observe donc un déplacement, le terme de comparaison n'est plus fondamentalement Dieu, et l'animal tend à lui disputer ce rôle. Davantage et de manière différente qu'auparavant, ce dernier est devenu un miroir de l'homme et c'est à ce titre qu'il occupe dans l'anthropologie naissante une place essentielle. Plus précisément, il acquiert ce rôle car il accompagne la formation d'une anthropologie nouvelle qui ne peut plus se fonder sur les termes de comparaison antérieurs. La nouveauté de cette anthropologie tient à six tendances, puisqu'il ne s'agit en effet que d'un ensemble de tendances et qui ne trouveront pas leur point d'aboutissement au XVIII ${ }^{\mathrm{e}}$ siècle même, mais se prolongeront bien au-delà :

En premier lieu, cette anthropologie n'est plus une anthropologie de la différence, mais une anthropologie de la différenciation. Désormais, c'est sur la base préalable d'une unité originaire que l'on cherche en un second temps à distinguer l'homme et l'animal, par un processus de transformation et non plus, comme auparavant, en les asseyant dès l'abord dans une différence figée (que ce soit sur la base d'une métaphysique dualiste des substances ou d'une métaphysique finaliste de type aristotélicien) à partir de laquelle certains traits communs se trouvent ensuite en général dégagés. La conception qui commence, difficilement et progressivement, à émerger est donc d'ordre génétique : l'animalité n'est plus simplement ce à quoi s'oppose l'humain pour se constituer, mais elle tend à être ce à partir de quoi l'humain s'édifie par réflexion et culture. Métaphysiquement, cela est lié à la critique du dualisme et à la recherche d'un principe d'unité ontologique générale, ce qui précipitera vers la recherche de l'origine. Celle-ci se révélera particulièrement problématique puisqu'il s'agira de la penser comme une, tout en admettant la nécessité d'une différenciation décisive à un moment ou un autre du processus, c'est-à-dire en fait de concevoir deux origines, sinon davantage, à partir d'une seule. Le problème dès lors ne sera plus celui de la coupure - celle entre l'homme et l'animal, mais également entre l'âme et le corps, l'esprit et la matière, etc. - mais celui plus délicat du seuil.

\footnotetext{
${ }^{1}$ Phrase tirée par Condillac de la première page du Discours sur la nature des animaux (t. II, p. 311).

${ }^{2}$ Pensée n 130-420.
} 
En deuxième lieu, cette anthropologie est traversée par un mouvement qui s'enracine en partie dans le siècle précédent, mais se développe au XVIII ${ }^{\mathrm{e}}$ : celui d'une révision à la baisse de la définition humaine. Les caractères innés - traditionnellement attribués à l'homme - de rationalité, de connaissance de Dieu, de sociabilité, de moralité ont tendance à diminuer (malgré, évidemment, bien des résistances). Depuis le XVII ${ }^{\mathrm{e}}$ siècle, est mis en avant le désir de conservation de soi, base unitaire de plus en plus consensuelle de l'anthropologie et de la compréhension du vivant en général. La nouvelle définition de l'humain, sous le signe d'une sorte de modestie métaphysique, tend à devenir minimale, à se régler par la sévérité d'une méthode radicale de dépouillement.

En troisième lieu, et cela joue un rôle majeur, s'opère une réévaluation des facteurs d'ordre empirique et matériel, particulièrement de la sensibilité sous sa forme physique, comme sensorialité, et corrélativement de la corporéité. Cette réévaluation est solidaire, en quatrième lieu, d'une promotion de la sensibilité cette fois sous sa forme morale, celle du désir, de l'affectivité, des passions, à l'encontre d'une certaine tradition chrétienne et déjà auparavant stoïcienne.

En cinquième lieu, il ne faut pas oublier également parmi tous ces facteurs la conception unitaire et non hiérarchique du monde que développe la science moderne. Pour celle-ci règne partout dans la nature l'unité des mêmes lois et, en conséquence, on ne peut trouver a priori de raisons pour que l'homme et l'animal relèvent ontologiquement de régimes différents.

Enfin, en sixième lieu, le plan métaphysique ou théologique - auparavant le plan premier dans lequel s'enracinait la définition de l'homme - s'éloigne et devient un arrière-plan (qui ne disparaît cependant pas pour autant : on peut donner comme exemple la métaphysique quelque peu fugitive de Condillac dans le Traité des animaux), tandis que l'anthropologie acquiert un statut premier et fondateur. On assiste donc à un renversement du rapport antérieur de la métaphysique et de l'anthropologie. Les appuis métaphysiques et théologiques du discours anthropologique ressemblent en effet de plus en plus à des gages de bonne conduite, prenant souvent l'allure plus ou moins de prétextes, ce qui est assez sensible par exemple chez Buffon. Ainsi, alors que la différence humaine avait toujours reposé sur un fondement métaphysique ou théologique, les Lumières initient un effort pour penser cette différence sur une base purement naturelle, en substituant dans ce domaine la question de l'origine à celle du fondement.

La spécificité humaine au XVIII ${ }^{\mathrm{e}}$ siècle se constitue dès lors de façon assez étrange, certes comme une nature, mais une nature en quelque sorte "en l'air », sans sol métaphysique ou théologique suffisamment consistant, et sans bases scientifiques encore assez établies; de là son apparence de construction finalement assez fragile. C'est dire l'importance, dans cet entre-deux inconfortable, d'appuis empiriques et comparatifs latéraux comme celui de l'animal. Cette fragilité de la fondation de la nature humaine au XVIII ${ }^{\mathrm{e}}$ siècle tient largement au fait que la catégorie d'histoire n'a pas encore acquis son caractère fondamental et autonome - ce qui sera le cas au siècle suivant. En outre, ce ne sera pas en se cantonnant au champ proprement historique, mais en s'étendant avec Boucher de Perthes au passé humain préhistorique, et avec Darwin à celui de la vie - et non plus essentiellement de la terre, comme avec Buffon, qui apparaît comme un maillon ${ }^{3}$

\footnotetext{
${ }^{3}$ En accroissant significativement - même si, bien sûr, c'est encore loin du compte - la durée de l'histoire de la terre.
} 
encore insuffisant, mais nécessaire - et de sa continuité phylogénétique. Cette catégorie d'histoire ainsi élargie et approfondie pourra prendre le relais de la notion de nature - en s'articulant d'abord à elle - pour donner plus de consistance à la nouvelle anthropologie. La formation de l'homme, en effet, sera alors dotée du recul temporel nécessaire permettant de la rendre effectivement concevable à partir d'une animalité originaire, ce qui n'était pas le cas auparavant.

Ces six aspects de la nouvelle anthropologie - différence par différenciation, révision à la baisse de la «nature humaine », promotion de la sensibilité physique et de la corporéité, promotion conjointe de la sensibilité morale et des passions, unité scientifique de la nature, retrait de la métaphysique - sont des facteurs puissants qui induisent fortement une requalification anthropologique de l'animalité. L'animal accroît sa pertinence en regard de l'homme, renforce l'effet de miroir, l'humain pouvant s'y étudier non pas simplement en négatif, pour ce qu'il n'est pas ou ne doit pas être, mais aussi de plus en plus pour comprendre son humanité même. Le mode de rapport en effet n'est plus seulement l'opposition comme c'était essentiellement le cas dans sa forme traditionnelle antérieure, mais bien aussi la saisie positive de la possibilité d'une certaine identification. L'animalité n'est plus non plus, comme au Moyen Âge, dégradation de l'humain dans la bestialité, comme recueil soigneusement répertorié des vices humains, ou à l'inverse sublimée dans un code allégorique des vertus chrétiennes ${ }^{4}$. Elle n'a plus un sens moral - négatif ou positif - mais celui d'un minimum naturel neutre qui n'est pas tant obstacle que base et condition avec quoi il faut compter. Cette sorte de neutralisation morale de l'animalité - solidaire, comme on l'a dit, d'une réévaluation des passions - qui s'accompagne d'un esprit beaucoup plus scientifique, est particulièrement évidente chez Hobbes. Mais si, au XVII ${ }^{\mathrm{e}}$ siècle, la comparaison reste encore très globale, mettant en jeu plus l'animalité que les animaux, au XVIII ${ }^{\mathrm{e}}$ en revanche, la surface de comparaison s'accroît. Non seulement l'animalité comme thème général semble davantage s'articuler à l'homme, mais en outre l'animal n'est plus seulement un thème général ou une idée, c'est de plus en plus aussi l'objet d'une étude positive et détaillée.

Or, tout cela induit un brouillage des frontières. Au siècle des Lumières, le miroir animal devient pour l'homme à la fois plus pertinent et plus trouble, comme amplifiant l'incertitude que Locke déjà, à la fin du siècle précédent, avouait à propos de la définition de l'homme en déclarant: "Nous sommes éloignés de connaître certainement ce que c'est qu'un homme $»^{5}$.

\section{Le brouillage des frontières}

Le brouillage des frontières homme/animal au milieu du XVIII ${ }^{\mathrm{e}}$ siècle tient donc pour une grande part - même s'il est loin de s'y épuiser - au renouvellement des données scientifiques depuis la fin du siècle précédent, et ce brouillage suscite régulièrement chez les savants - comme en écho au propos de Locke - la forme récurrente et assez étonnante d'un aveu d'incapacité à distinguer l'homme et l'animal sur une base purement

\footnotetext{
${ }^{4}$ Par exemple le symbole connu du pélican - supposé nourrir ses petits de ses entrailles - comme figure du dévouement parental.

${ }^{5}$ Cf. Locke, 1983, III, 6, 27, et III, 6, 22, p. 367, paragr. 27.
} 
scientifique, cela dans tous les domaines concernés. La différence alors se logera dans des déclarations métaphysiques et théologiques de principe pas toujours, comme on l'a vu, très convaincantes. Enumérons ces domaines.

En premier lieu, l'anatomie comparée dont les progrès permettent une compréhension de plus en plus grande de l'unité des organismes ${ }^{6}$. Buffon par exemple y insistera en montrant que c'est de façon périphérique, à mesure qu'on s'éloigne du centre, que se différencient les organismes. Ainsi, le Discours sur la nature des animaux, qui ouvre le quatrième volume de l'Histoire naturelle en 1753, déclare : «De plus, si nous y faisons attention, nous verrons que les plus grandes différences sont aux extrémités, et que c'est par ces extrémités que le corps de l'homme diffère le plus du corps de l'animal [...] En prenant donc le cœur pour centre dans la machine animale, je vois que l'homme ressemble parfaitement aux animaux par l'économie de cette partie et des autres qui en sont voisines; mais plus on s'éloigne de ce centre, plus les différences deviennent considérables, et c'est aux extrémités qu'elles sont le plus grandes » (Buffon, t. II, p. 315, dans l'éd. Flourens).

Dans la reconnaissance de cette unité, un pas décisif a été franchi avec Edward Tyson et la dissection du chimpanzé. En 1698, Edward Tyson et William Cowper présentent à la Royal Society de Londres leur rapport de dissection (publié en 1699) sur un «orangoutang » (en fait un chimpanzé). La conclusion de Tyson est que l'animal présente une affinité remarquable avec l'organisme humain, bien plus que tous les autres animaux - même les singes - connus jusqu'à présent, mais, ajoute-t-il de façon tout à fait cartésienne, puisqu'il est incapable de parler de son vivant alors qu'il possède tous les organes pour cela, cela établit que, métaphysiquement, l'homme possède un statut à part (un esprit). Le rapport de Tyson, catalogue extrêmement détaillé des ressemblances et des dissemblances, mais trop partial, appuyant davantage sur les premières que sur les secondes (notamment à l'égard du cerveau), aura - couvert par sa grande réputation et répercuté par Buffon s'appuyant sur son autorité ${ }^{7}$ - des effets qui persisteront tout le long du siècle.

Il faudra attendre quatre-vingts ans, en 1779, pour que le travail et les conclusions de Tyson soient scientifiquement critiqués par le Hollandais Camper qui, disséquant à nouveau des pongidés, mettra en évidence des sacs laryngiens rendant impossible l'articulation des sons et donc le langage. Signalons que les pongidés de Camper étaient des orangs-outangs véritables, c'est-à-dire asiatiques, alors que Tyson avait eu affaire à un chimpanzé, lequel n'est jamais équipé de tels sacs. Cependant, Camper pointait également d'autres éléments qui étaient en revanche tout à fait pertinents et qui révélaient une différence anatomique entre l'homme et les grands singes bien plus grande que celle concédée par Tyson. Le «malentendu » suscité par Tyson d'une proximité scientifiquement indiscutable, et touchant presque à l'identité, du moins au plan physique, commence alors sérieusement à se dissiper. Toutefois, l'affaire est loin d'être terminée et cet épisode

\footnotetext{
${ }^{6}$ Celle-ci aboutira au siècle suivant à l'unité de plan du règne animal défendue par E. Geoffroy Saint-Hilaire et combattue par Cuvier.

${ }^{7}$ « Comme quelques-uns des faits que nous venons d'exposer pourraient paraître suspects à ceux qui n'auraient pas vu cet animal, nous avons cru devoir les appuyer de l'autorité de deux célèbres anatomistes, Tyson et Cowper, qui l'ont ensemble disséqué avec une exactitude scrupuleuse, et qui nous ont donné les résultats des comparaisons qu'ils ont faites de toutes les parties de son corps avec celles de l'homme. ", "Les orangs-outangs », Histoire naturelle, t. IV, dans l'éd. Flourens, pp. 33-34.
} 
entre dans une série de vives controverses ostéologiques, une véritable «bataille de l'os ». Marquée en France par l'article en 1764 de Daubenton prouvant, grâce au trou occipital $^{8}$, la destination bipède de l'homme et celle globalement quadrupède des autres mammifères, elle se poursuit par Camper encore qui, sur la base toujours de la dissection du pongo, estime établir l'absence de l'os intermaxillaire supérieur chez l'homme alors que tous les autres animaux - même en l'espèce les pongos - en sont pourvus, ce qui atteste à ses yeux leur différence de nature.

Cependant, un nouveau rebondissement se produit en Allemagne : Goethe, en désaccord avec Camper, découvre en 1786 cet os intermaxillaire ${ }^{9}$ chez l'homme, précieux sésame réinsérant à nouveau l'homme dans le monde animal. L'ostéologie n'est qu'une pièce, certes très stratégique, dans un vaste débat anatomique dont les cibles et enjeux privilégiés sont essentiellement: l'exclusivité de la bipédie chez l'homme (pour ou contre laquelle l'argumentation anatomique n'emprunte pas qu'à l'ostéologie: par exemple, l'adhérence du péricarde et du diaphragme permettant le maintien des viscères en position debout est un élément important), la phonation (l'appareil pharyngo-laryngé en premier lieu), et la pensée (avec en particulier le cerveau).

En second lieu, la classification, spécialement celle linnéenne, Linné effectuant une sorte de régularisation taxinomique de l'homme. Dans la dixième édition du Systema Naturae (1758), il présente ce dernier comme constituant le genre Homo rangé dans l'ordre plus vaste des primates. Il distingue dans ce genre Homo deux types : l'Homo sapiens ou diurnus (où à côté des races européenne, américaine, asiatique et africaine, on trouve aussi l'Homo ferus, c'est-à-dire l'homme ensauvagé, retourné à la pure nature, ainsi d'ailleurs que des formes tout à fait fantastiques) et l'Homo sylvestris (ou Homo nocturnus, où se situe l'orang-outang), genre intermédiaire entre l'homme et le singe. Explicitement, même si ce n'est qu'au plan de la nomenclature, l'homme était intégré dans le monde animal. Linné ira très loin en ce sens puisqu'il reconnaît en 1746 dans la Fauna Suecica : «Il est vrai qu'en conséquence de mes principes d'histoire naturelle, je n'ai jamais su distinguer l'homme du singe ${ }^{10}$. Buffon, inspiré par Tyson, le rejoint partiellement quelques années après, en 1766, dans sa Nomenclature des singes, sous la forme révélatrice encore d'un aveu : « Je l'avoue, si l'on ne devait juger que par la forme, l'espèce du singe pourrait être prise pour une variété dans l'espèce humaine : le Créateur

\footnotetext{
${ }^{8}$ Cf. Daubenton, 1764, pp. 568-575. Ce trou à la base du crâne permet chez l'homme l'insertion de la colonne vertébrale grâce à un angle avec cette dernière rendant possible la bipédie, à la différence des autres animaux chez qui le même angle la rend impossible, même chez les pongidés, en tout cas comme attitude aisée et permanente.

${ }^{9}$ Pour expliquer l'étrange incertitude - cause de tous ces retournements - de ce qui devrait être une simple constatation de fait, il faut préciser que cet os n'en est en réalité pas vraiment un, mais une sorte de pièce de la mâchoire supérieure (portant les incisives) très vaguement visible chez l'homme (il l'est nettement en revanche chez les herbivores) par des sutures peu apparentes et s'effaçant avec l'âge (précisément comme chez les grands singes, d'où d'interminables atermoiements).

${ }^{10}$ Cité par Diderot dans la Pensée 49 des Pensées sur l'interprétation de la nature (1754) : «L'homme, dit Linnaeus (Fauna Suecica, préf.), n'est ni une pierre, ni une plante ; c'est donc un animal. Il n'a pas un seul pied ; ce n'est donc pas un ver. Ce n'est pas un insecte, puisqu'il n'a point d'antennes. Il n'a point de nageoires ; ce n'est donc pas un poisson. Ce n'est pas un oiseau, puisqu'il n'a pas de plumes. Qu'est-ce donc que l'homme ? il a la bouche du quadrupède. Il a quatre pieds ; les deux de devant lui servent à l'attouchement, les deux de derrière au marcher. C'est donc un quadrupède. » " Il est vrai, continue le méthodiste, qu'en conséquence de mes principes d'histoire naturelle, je n'ai jamais su distinguer l'homme du singe» (1994, pp. 586-587, mis en italiques par nous).
} 
n'a pas voulu faire pour le corps de l'homme un modèle absolument différent de celui de l'animal ; il a compris sa forme, comme celle de tous les animaux, dans un plan général ; mais en même temps qu'il lui a départi cette forme matérielle, semblable à celle du singe, il a pénétré ce corps animal de son souffle divin » (t. IV dans l'éd. Flourens, pp. 17-18) et, un peu plus haut, il avait déclaré : « On verra, dans l'histoire de l'orang-outang, que, si l'on ne faisait attention qu'à la figure, on pourrait également regarder cet animal comme le premier des singes ou le dernier des hommes, parce qu'à l'exception de l'âme, il ne lui manque rien de tout ce que nous avons, et parce qu'il diffère moins de l'homme pour le corps, qu'il ne diffère des autres animaux auxquels on a donné le même nom de singe. » (t. IV dans l'éd. Flourens, p. 16). Mais Buffon qualifie ensuite cette apparence d'humanité chez les singes en général de sorte de "masque ${ }^{11}$ dissimulant la plus grande dissemblance de fond : cela certes, il l'affirme du singe, mais l'orang-outang, précise-t-il, incapable de langage, n'est bien qu'un singe.

En troisième lieu, on voit l'importance du point que l'on aborde maintenant, à savoir la « découverte » des pongos (c'est-à-dire des grands singes, appelés depuis Pongidés), découverte certes éthologiquement encore très insuffisante, d'où les récriminations de Rousseau dans la note $\mathrm{X}$ du Discours sur l'origine et les fondements de l'inégalité parmi les hommes. En attendant, les pongidés ramenés en Europe au XVIII ${ }^{\mathrm{e}}$ siècle ont une proximité avec l'homme si frappante que - anecdote connue de l'époque ${ }^{12}$ - devant l'un d'eux, un orang-outang au Jardin du roi, un cartésien aussi convaincu que le cardinal de Polignac se serait écrié : «Parle et je te baptise !», propos singulier par la manière dont, à la fois, il exprime étonnement devant la ressemblance et parfaite assurance quant à la différence. Il s'agit en effet d'un étonnement car, auparavant, l'Antiquité et le Moyen Âge ont pratiquement ignoré les singes anthropomorphes (à part quelques exceptions comme celle du périple du carthaginois Hannon vers le $\mathrm{VI}^{\mathrm{e}}$ siècle avant J.-C.). Le fait est très important car les singes connus dans l'Antiquité inquiétaient beaucoup moins la spécificité humaine : leur ressemblance avec l'homme est en effet bien plus éloignée que celle des pongos, entre autres parce qu'ils sont clairement quadrupèdes ou plutôt quadrumanes (comme y insiste Buffon après Tyson). Aux XVI ${ }^{\mathrm{e}}$ et $\mathrm{XVII}^{\mathrm{e}}$ siècles encore, malgré quelques rares exemplaires parvenus en Europe, c'est surtout par l'intermédiaire des récits de voyage et en particulier des croquis que l'on a $\mathrm{pu}$ en faire ${ }^{13}$ que l'on en prend connaissance, croquis en règle générale très fortement anthropomorphisés, notamment pour le visage et la station droite. Au XVIII ${ }^{\mathrm{e}}$ siècle en revanche devient possible un accès plus direct, en particulier à la foire Saint-Germain (où un « orang-outang » fut exposé en 1720 (de Maillet, 1984, p. 270)), à la ménagerie de Versailles et dans le Jardin du roi, même si cela reste tout de même extrêmement

\footnotetext{
${ }^{11}$ «Aussi ce singe [...] n'est dans la vérité qu'un pur animal portant à l'extérieur un masque de figure humaine », (t. IV dans l'éd. Flourens, p. 22).

${ }^{12}$ Rapportée par exemple par Diderot dans Le rêve de d'Alembert : «- Bordeu : Avez-vous vu au Jardin du Roi, sous une cage de verre, cet orang-outan qui a l'air d'un saint Jean qui prêche au désert ? - Mademoiselle de L'Espinasse : Oui, je l'ai vu. - Bordeu : Le cardinal de Polignac lui disait un jour : Parle, et je te baptise. », p. 675.

${ }^{13}$ En particulier ceux des Hollandais Tulpius et Bontius, tous les deux autour de 1630, le premier en Europe sur la base d'un exemplaire mort (sans doute un chimpanzé, mais sans certitude possible), le second à Java. Sur toutes ces questions, $c f$. Franck Tinland, 2003, et Claude Blanckaert, 1991.
} 
difficile ${ }^{14}$. Par ailleurs, les grands singes en question que l'on observe ne sont connus que partiellement puisque ce sont seulement des chimpanzés et des orangs-outangs, que l'on confond d'ailleurs ensemble sous ce dernier vocable, et le gorille n'est pas encore identifié.

Or, c'est justement essentiellement ce dernier dont parle Rousseau sous le nom de pongo dans la note $\mathrm{X}$ du second Discours. Cependant, à l'inverse de ce que cet auteur envisageait au moins comme possible, leur connaissance plus approfondie en réduira les performances supposées et alimentera un mouvement de réaction, la découverte scientifique du gorille au milieu du XIX ${ }^{\mathrm{e}}$ siècle (1847) verrouillant finalement ce retour en arrière : l'animal qui était qualifié d'anthropomorphe sera bestialisé (pour le grand public en gros jusqu'à Diane Fossey et la popularisation de son œuvre et de sa vie dans les années 1980). Plus précisément, cette « réduction » scientifique du pongo se fera par un double biais, anatomique et particulièrement ostéologique (ce que l'on a déjà évoqué) d'une part et éthologique de l'autre, à partir du dernier tiers du XVIII ${ }^{\mathrm{e}}$ siècle $^{15}$. Ces deux approches convergent pour rectifier l'allure si anthropomorphe auparavant du pongo en établissant sa station semi fléchie. Cependant, un certain relais sera assez vite assuré par le darwinisme qui transférera la projection anthropomorphique sur l'ancêtre primate supposé de l'homme.

Évoquons plus rapidement le quatrième domaine, celui de la physiologie, où se mènent des recherches, d'une part, sur la génération et l'embryologie - auxquelles on lie alors celles sur la génération spontanée, les régénérations diverses (le polype de Tremblay), l'hérédité et la tératologie - et de l'autre, sur le système nerveux, les tissus et les organes ; travaux donnant lieu à la réaction animiste de Stahl contre le mécanisme, à la « découverte » de l'irritabilité par Haller, et enfin à l'extension de la sensibilité avec l'École de Montpellier. Toutes ces recherches alimentent plus ou moins la tendance très forte à un nouveau vitalisme - nouveau car fondé sur ces données scientifiques - qui s'impose à partir du milieu du siècle et offre une vision continuiste de la vie et des vivants mise à profit par les matérialistes pour étayer la proximité de l'homme et de l'animal.

Signalons enfin, toujours trop brièvement, l'étude également essentielle de ce qu'on peut appeler les « marges anthropologiques ». Il en est ainsi par exemple des handicaps. Le cas est fameux du sourd de Chartres (dont l'histoire est rapportée entre autres par Fontenelle et La Mettrie (2004, p. 56)) qui, un beau jour, recouvra l'ouïe tout à fait par hasard grâce au son des cloches de la cathédrale. Le récit qu'il fit de sa vie avant

\footnotetext{
${ }^{14}$ En fait foi par exemple cette déclaration en 1764 d'un naturaliste pourtant aussi chevronné que Daubenton : « Je n'ai point vu de jocko vivant » (« Sur les différences de situation du grand trou occipital dans l'homme et dans les animaux », pp. 568-575). Sa connaissance directe des grands singes, au moins jusqu'à cette date, et au moins pour le jocko (c'est-à-dire le chimpanzé), reste donc exclusivement anatomique, comme chez la plupart des savants qui en parlent. Deux ans après, en 1766, sera publiée La nomenclature des singes où Buffon fait état d'une dissection de chimpanzé effectuée en commun avec Daubenton et où il déclare avec une singulière insistance (cette déclaration effectuée dès l'abord est répétée presque à chaque page de l'article sur les orangsoutangs, ce qui marque bien la rareté de la chose) avoir « vu le petit orang-outang ou le jocko vivant », p. 23.

${ }^{15}$ Ainsi, le même Diderot qui soulignait implicitement en 1769 la ressemblance troublante du pongo et de l'homme dans Le rêve de d'Alembert, écrira-t-il ensuite (sans doute vers la fin des années 1770) dans les Eléments de Physiologie: "J'ai vu un homme singe : il ne pensait pas plus que le singe, il imitait comme le singe, il était malfaisant comme le singe, il ne parlait point, mais il jetait des cris comme le singe ; il s'agitait sans cesse comme le singe ; il se fâchait, il s'apaisait, il était sans pudeur comme le singe », Euvres, t. I, p. 1278.
} 
cet événement est celui d'une vie réduite aux seules sensations : le fond originaire de l'humain semble alors se révéler comme purement animal et la spécificité humaine n'avoir plus rien d'inné. La réflexion également sur les enfants sauvages, auxquels les philosophes prêtent davantage attention au XVIII ${ }^{\mathrm{e}}$ siècle, tend de plus en plus à des conclusions dans le même sens.

\section{Les spéculations}

Ces avancées scientifiques sont largement utilisées par diverses spéculations qui renforcent ce rapprochement de l'homme et de l'animal. On peut les classer en quatre rubriques.

\section{Du côté des métaphysiques matérialistes}

C'est ce qu'on peut appeler l' " extrémisme matérialiste », ce qui renvoie à La Mettrie, Diderot, Helvétius, d'Holbach pour citer les plus connus. Certes, ils ont toujours plus ou moins rapproché l'homme et l'animal, mais l'élément nouveau, c'est que le rapprochement qu'ils effectuent ne s'apparente plus au XVIII ${ }^{\mathrm{e}}$ siècle à une stratégie sceptique (rejoignant ainsi un camp qui déborde la sphère de la stricte obédience matérialiste), comme on la trouve par exemple encore chez Montaigne, argumentation s'alimentant souvent - de façon sans doute en général plus tactique que sincère - au merveilleux naïf et crédule des récits et des légendes. Ce rapprochement s'étaye désormais essentiellement de données scientifiques nouvelles - l'anatomie et la physiologie comparées, la classification, l' « éthologie » naissante et la psychologie animale ${ }^{16}$, toutes deux bien sûr encore tout à fait embryonnaires - et également des nouvelles théories de la connaissance. Les matérialistes vont donc instrumentaliser la question de l'animal - auquel ils s'intéressent en général moins pour lui-même qu'essentiellement dans une optique stratégique de déstabilisation des privilèges humains, de l'âme spirituelle et en particulier de la croyance au libre arbitre - en s'appuyant sur ces nouvelles bases du savoir, la science constituant pour eux un véritable tremplin spéculatif. Le mouvement est par conséquent beaucoup plus argumenté, voire dogmatique, agressif et donc dangereux. C'est de plus un nouveau type de matérialisme qui tend à s'imposer : le matérialisme vitaliste - dont Diderot est le fer de lance - qui, très logiquement, fait constamment appel, de façon systématiquement alternée ou entrelacée, aux données physiologistes et à celles concernant les animaux. Chez certains matérialistes cependant, l'animal, sous l'angle de cet intérêt pour la vie et le vivant - intérêt lui-même lié à la recherche métaphysique sur la nature et l'origine matérielle de toutes choses -, reçoit une attention plus directe et plus puissante, moins instrumentalisée ; le corps animal vivant et sentant apparaît comme l'une des portes d'entrée essentielles pour toutes ces questions.

Ces spéculations matérialistes mettent en outre en lumière le rôle des sens et de la corporéité : la main notamment est consacrée comme véritable clé biologique (comme par exemple chez Helvétius) du devenir humain. L'animal devient alors une sorte de statue physiologique et expérimentale « grandeur nature » pour beaucoup de ces auteurs.

\footnotetext{
${ }^{16}$ Spécialement celle des primates : c'est le cas par exemple de La Mettrie qui réclame l'enseignement du langage aux singes.
} 
On peut, en ajoutant ou retranchant tels et tels organes, composer ou décomposer hommes et animaux, l'animalité se convertissant en système de transformations se prêtant à la fois à l'observation empirique et à l'expérimentation mentale. Chez les matérialistes, la comparaison débouche ainsi sur la combinaison et la différence humaine se résout dans ses éléments animaux, l'humain n'étant plus rien d'autre qu'une combinaison animale particulièrement réussie, comme par exemple chez Diderot.

\section{Du côté des théories de la connaissance}

Ici, se distingue le « radicalisme épistémologique» de Condillac. Celui-ci n’est pas matérialiste, mais tout à fait spiritualiste. Cependant, comme sensationniste, il rejoint les matérialistes dans leur insistance sur le rôle des sens, de la main et du toucher, de la corporéité en général. Le processus de construction de la connaissance est pour lui fondamentalement unitaire et c'est à partir de ce courant sensible commun que l'animal et l'homme, à un certain moment, vont diverger du fait de différences dans l'équipement organique (c'est essentiellement le rôle de la main et du toucher chez l'homme) et dans les besoins. Mais Condillac maintient une différence métaphysique de principe. Cette différence est évidemment supprimée dans la version matérialiste du sensationnisme. De manière générale, on peut observer la tendance au milieu du siècle, à passer de l'empirisme lockien, admettant l'origine indépendante de la réflexion et du jugement, donc encore cartésien par ce côté, à un sensationnisme radical étendant l'origine sensible à toute la sphère de l'esprit, qu'il s'agisse de celui de l'homme ou de l'animal. La théorie de la connaissance pousse donc ici au rapprochement de l'homme et de l'animal.

\section{Du côté de l'origine}

Ici, se signale l'audace des spéculations sur l'histoire de la nature - donc à propos de la question décisive du temps (on a vu le rôle de Buffon sur ce point) - et sur l'origine de l'homme en poussant la notion d'état de nature plus loin que cela n'avait été fait jusque-là pour arriver jusqu'à sa limite ultime. Concernant ce dernier point, Rousseau est central. Ce dernier en effet dépouille l'homme de l'état de nature davantage qu'aucun de ses prédécesseurs en lui retirant non seulement la sociabilité comme Hobbes, mais également toute rationalité (alors que Hobbes lui laissait une capacité minimale de calcul), tout développement affectif et toute existence familiale. Il le rétrograde à un état animal tel que plus rien extérieurement ne le distingue des animaux. Certes, Rousseau invoque une différence métaphysique, celle de la liberté de la volonté, mais pour ajouter tout de suite qu'étant inobservable, elle prête indéfiniment à discussion. Il éprouve par conséquent le besoin de la relayer par un critère observable et empirique, celui de la perfectibilité. Mais celle-ci étant en principe non encore vraiment actualisée dans le pur état de nature, elle y demeure donc en principe également invisible. Même si Rousseau se garde bien de franchir le Rubicon, l'homme de l'origine apparait extérieurement comme indiscernable des animaux. C'est cet excès d'audace qu'on lui reprochera, comme si, malgré toutes ses précautions, il avait transgressé une limite interdite. Après Rousseau et cette hypothèse de l'état animal de nature, il ne restera plus, il est vrai, qu'à aller encore un peu plus loin pour passer, par la paléoanthropologie naissante, de l'état animal d'origine de l'homme à la pure et simple origine animale de l'homme. 


\section{Du côté de la psychologie humaine}

C'est la revalorisation de la sensibilité et des passions qui nous donnera encore l'occasion de reparler de Rousseau. On trouve la théorie des passions, et aussi, dans une certaine mesure, l'éthique (avec l'effort pour trouver un fondement naturel de la morale). En la matière se rencontrent assez naturellement chez les matérialistes de fréquents rapprochements entre l'homme et l'animal, mais ces rapprochements sont loin de s'effectuer seulement sous leur bannière. Ainsi, Rousseau, qui pourtant s'oppose aux matérialistes, emprunte constamment aux animaux pour construire son anthropologie. Par exemple, dans l'Émile, il trace régulièrement un parallèle avec l'animal pour comprendre l'enfant, ses besoins, ses réactions, ses tendances ${ }^{17}$. Il peut s'observer ainsi des sentiments innés plus ou moins communs entre l'homme et les animaux : ce sont l'amour de soi, la pitié, la paresse, la sexualité, la pudeur, l'imitation. Ainsi, par exemple, la compréhension de la sexualité chez l'homme bénéficie de la comparaison animale. En effet Rousseau tire argument de celle des animaux pour s'opposer à l'idée de nombre d'auteurs à son époque (La Mettrie, Diderot, Buffon par exemple) qui lui accordent une puissance naturelle qu'il juge disproportionnée. Il fait remarquer que la sexualité n'est précisément pas si importante chez les animaux, ce qui selon lui indique l'origine culturelle du développement particulier qu'elle prend chez l'homme. Ces sentiments innés, en particulier la pitié, fournissent chez Rousseau l'appui naturel de la morale qui cependant ne devient telle que lorsqu'elle se trouve ressaisie par la réflexion. Selon sa perspective, l'homme, loin de se définir unilatéralement par opposition à l'animal, constitue au contraire son humanité sur la base de son unité réfléchie avec lui.

Ainsi, le rapport à l'animal nous semble bien représenter, sinon le rapport essentiel, du moins un creuset privilégié de la nouvelle anthropologie qui émerge sous les Lumières, un pivot fondamental de comparaison par lequel l'homme s'expérimente dans la nouvelle compréhension qu'il a de lui-même, et où en même temps il éprouve le vertige de ressentir une différence, naguère grosso modo métaphysiquement et théologiquement assurée, problématique et incertaine comme jamais. Du caractère inquiétant de ce jeu si serré d'étrangeté et d'identité, le célèbre tableau de Chardin - La raie - peut certainement prétendre à être considéré comme l'emblème ${ }^{18}$. Le miroir animal de l'homme des Lumières, loin d'offrir un reflet tranquille de soi, est mis en abîme de soi et tend à terme - terme qui se situe dans le siècle suivant avec Lamarck et Darwin - à montrer l'homme comme essentiellement transformation animale. Certes, il faut nuancer, ce mouvement de rapprochement de l'homme et de l'animal non seulement est inabouti, mais également très relatif. L'homme du XVIII ${ }^{\mathrm{e}}$ siècle en général demeure au fond plus centré sur son rapport à l'animal que sur l'animal lui-même, la preuve en est le classement délibérément anthropocentré choisi par Buffon pour son Histoire naturelle, l'ordre d'examen des espèces s'y réglant sur l'utilité humaine. Cet homme ainsi se pose fréquemment comme avant tout dominateur du monde animal, avec une marge importante de tranquillité et de

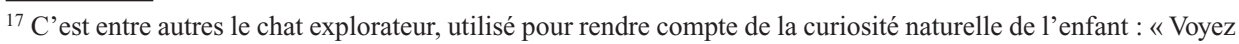
un chat entrer pour la première fois dans une chambre; il visite, il regarde, il flaire, il ne reste pas un moment en repos, il ne se fie à rien qu'après avoir tout examiné, tout connu. Ainsi fait un enfant commençant à marcher, et, entrant pour ainsi dire dans l'espace du monde » (Rousseau, 1969).

${ }^{18}$ Nous nous permettons sur ce point de renvoyer à notre article: Guichet, «L'animal dans la peinture de Chardin », 2004.
} 
satisfaction de soi, sans apparemment d'interrogation excessive. Cependant, une certaine contenance affichée à l'égard de soi ne signifie pas que, en profondeur, il ne se passe rien et que les rapports superficiellement exhibés n'y soient pas en crise et en interrogation, ce dont on a cherché dans cet article à montrer les signes. De même, le fait que ce rapprochement soit fondamental ne veut pas dire qu'il soit exclusif. Si ce rapport à l'animal nous semble être au moins aussi essentiel, si ce n'est davantage, que les autres facteurs - ces autres facteurs étant la comparaison ethnologique (et, de plus en plus, raciologique ${ }^{19}$ ) grâce aux récits de voyage, la réflexion sur les handicaps et les monstres, et enfin celle sur les enfants sauvages, c'est-à-dire l'ensemble de ce qu'on peut appeler les marges anthropologiques -, ces facteurs comptent bien sûr également de façon décisive. Mais le rapport à l'animal se situe dans les départages fondamentaux où l'homme doit décider de lui-même ce qu'il estime le plus constitutif de sa spécificité et son unité, tandis que les autres rapports mentionnés interviennent davantage à un niveau ultérieur, interhumain. Cependant, cette continuité pressentie de l'homme et de l'animal au XVIII ${ }^{\mathrm{e}}$ siècle manque encore d'un maillon essentiel, celui de la démonstration scientifique de la continuité phylogénétique, laquelle, débutant véritablement avec Lamarck, ne sera effectivement constituée qu'au milieu du XIX ${ }^{\mathrm{e}}$ siècle avec Darwin. Faute du maillon décisif de cette continuité qu'on pourrait appeler « bio-historique », la nouvelle conception n'offre pas de capacité de résistance suffisante à des réactions qui se durcissent dans le dernier tiers du XVIII ${ }^{\mathrm{e}}$ et la première moitié du XIX ${ }^{\mathrm{e}}$, donnant lieu notamment aux vives controverses ostéologiques, celles de la «bataille de l'os », déjà évoquées. Cette première moitié du $\mathrm{XIX}^{\mathrm{e}}$ siècle voit se constituer une nouvelle synthèse alliant les nouvelles données scientifiques, anatomiques et «prédiluviennes »-qui s'accumulent sans cadre transformiste encore assez crédible et accepté qui permettrait de les rendre intelligibles - à des préoccupations d'ordre spiritualiste veillant à préserver une coupure ontologique tout à fait tranchée entre l'homme et l'animal : cette synthèse, préparée dans une certaine mesure par Buffon et d'autres, c'est celle - magistrale - de Cuvier.

\footnotetext{
$\overline{19}$ Où, cependant, l'animal se rappelle à nouveau dans la mesure où il entre de plus en plus dans le discours de l'homme sur l'homme, comme c'est exemplairement le cas avec Camper dont l'angle facial servira d'instrument de mesure pour évaluer l'indice d'animalité des diverses «races» humaines. Au siècle suivant ainsi, l'unité de l'homme et de l'animal sera largement utilisée pour séparer l'homme de l'homme. C'est là la récupération raciste du continuisme, ce qui ne veut pas dire que c'en soit le destin.
} 


\section{Chapitre 7 Humanité et animalité dans la pensée humaniste}

JEAN-PIERRE SYLVESTRE

Dans son ouvrage, Par-delà nature et culture (2005), l'anthropologue Philippe Descola nous invite à interpréter la représentation que les sociétés humaines ont de l'animalité en fonction de leur conception de la nature. On peut considérer, selon lui, qu'il en existe quatre grands types ontologiques qu'il dénomme respectivement animique, totémique, analogique et naturaliste. Ceux-ci correspondent à une structuration de l'expérience individuelle et collective se ramenant à un double schéma d'identification des êtres non humains qui gouverne la relation entretenue avec eux ${ }^{1}$.

Le naturalisme, que nous préférons appeler « humanisme », pour éviter toute confusion avec le «physicalisme » ou le «biologisme», caractérise la culture occidentale moderne, laquelle établit une différence générique entre les mondes animaux et le monde humain fondée sur la présence de l'esprit, propre à ce dernier. Ce qui différencie les humains des non-humains pour notre culture, c'est la conscience intentionnelle ou subjectivité, c'est-à-dire le pouvoir de signifier par la maîtrise des symboles et du langage. Autrement dit, si nous reconnaissons bien notre appartenance au monde animal par notre nature physique et biologique, nous considérons que nous nous en séparons radicalement par notre intériorité psychique et sa double dimension rationnelle et morale qui fait de nous des êtres substantiellement autonomes. En découle une définition de la

\footnotetext{
${ }^{1}$ « La distribution des quatre combinaisons de ressemblances et de différences s'organise à partir de deux axes verticaux. L'un est caractérisé par de grands écarts dichotomiques, par la prééminence du continu sur le discontinu et par l'inversion des pôles d'inclusion hiérarchique : la continuité des intériorités entre humains et non-humains partageant une même "culture" prend dans l'animisme la valeur de l'universel (par contraste avec le particulier et le relatif qu'introduisent les différences de forme et d'équipements biologiques), tandis que c'est la continuité des physicalités dans le champ de la "nature" qui joue ce rôle dans le naturalisme (par contraste avec le particulier et le relatif qu'introduisent les différences culturelles). L'autre axe privilégie les contiguïtés chromatiques et juxtapose en une symétrie couplée un système de ressemblances tendant vers l'identité, le totémisme, et un système de différences graduelles tendant vers la continuité, l'analogisme » (Descola, 2005, pp. 321-322).
} 
subjectivité humaine à partir de deux traits essentiels bien mis en évidence par la philosophie criticiste : la " réflexivité », ou capacité de travail lucide sur soi d'une part, et la « volonté », ou capacité d'action délibérée d'autre part. La réflexivité n'est pas seulement l'aptitude à l'autoréférence, présente dans tout être vivant, mais l'aptitude d'un sujet à se mettre à distance de soi, à se poser comme objet de sa propre intellection. Quant à la volonté, elle est la dimension réfléchie de notre essence créatrice, l'expression de notre faculté à conduire notre existence en fonction de projets qui ne sont pas réductibles à la continuation complexifiée de nos pulsions instinctuelles.

Une telle vision du monde n'est-elle, comme le pense Descola, qu'une des déclinaisons possibles de notre rapport à l'être ? N'est-elle ni plus ni moins légitime que les trois autres ontologies répertoriées, quels que soient par ailleurs ses incontestables mérites, notamment dans les domaines scientifique ou politique, et le fait que nous ne puissions pas nous soustraire du « terreau familier du naturalisme » (Descola, 2005, p. 418), acquis et fortifié pendant notre socialisation ${ }^{2}$ ? Faut-il considérer, par exemple, que l'ontologie des sociétés animistes pour lesquelles la catégorie des «personnes » englobe des esprits, des plantes et des animaux, tous dotés d'une âme, possède une valeur de vérité aussi probante que celle de la modernité occidentale, dans la mesure où elle est l'une des expressions possibles des modes d'objectivation symbolique du monde et d'autrui? Faut-il même aller jusqu'à supposer qu'elle possède une sorte de probité ou de sagesse supérieure à la nôtre, que retrouveraient indirectement ceux de nos contemporains qui, scientifiques, philosophes ou militants de la «cause animale », insistent sur le caractère ténu des frontières qui séparent l'humanité de l'animalité et incitent par là même à reconsidérer l'ensemble de nos relations avec nos « frères inférieurs »?

À contre-courant de ces idées, dont nous ne sous-estimons pas pour autant l'intérêt et l'importance, notre propos est de défendre l'idée, anti-relativiste, que la pensée humaniste, en établissant une différence de nature, et non pas seulement de degrés, entre l'homme et l'animal, n'exprime pas exclusivement les valeurs et les convictions d'une culture particulière, mais développe un niveau de réflexion et d'argumentation inégalé pour nous faire accéder à la connaissance vraie des ordres humain et animal.

Nous procéderons en trois temps. Tout d'abord, nous rappellerons les thèses kantiennes et fichtéennes en faveur de la spécificité de l'ordre humain. Ensuite, nous montrerons leur adéquation avec des recherches récentes de l'éthologie. Enfin, en nous appuyant sur les travaux de Cornélius Castoriadis qui, à bien des égards, prolongent et approfondissent la pensée criticiste, nous nous efforcerons de mettre en évidence que c'est l'imagination créatrice, comme dimension spécifique de l'autonomie, qui permet d'identifier et de justifier la singularité radicale de l'univers humain.

\footnotetext{
${ }^{2}$ Dans l'universalisme relatif, dont il se réclame, Descola défend un programme anthropologique, d'inspiration structuraliste, qui requiert seulement « un sujet qui ne préjuge pas du vécu de la conscience d'autrui à partir du vécu de sa conscience et qui admet néanmoins que le monde offre à tous le même genre de "prises", quels que soient les usages, cognitifs et pratiques, auxquels ils se prêtent ; un sujet plus attentif au réel institué par l'activité intentionnelle des subjectivités très diverses dont il étudie les produits qu'aux évidences trompeuses de sa propre intentionnalité instituante, dès lors ramenée aux dimensions d'un filtre imparfait, toujours contaminée par des déterminations historiques qu'aucune épochè ne saurait réduire ; un filtre certes indispensable mais qui n'a d'autre privilège que d'être le seul accessible, et qu'il doit parvenir à objectiver de l'extérieur comme une simple variation parmi d'autres du régime de l'être, provisoirement investie d'une fonction de connaissance totalisatrice en raison de la conjoncture où il se trouve placé » (Descola, 2005, p. 419 ; cf. aussi p. 131).
} 


\section{La spécificité de l'ordre humain dans la pensée criticiste : les arguments de Kant et de Fichte}

Pour la pensée criticiste, entre l'ordre animal et l'ordre humain la différence n'est pas seulement de degrés mais de nature, avons-nous dit. Celle-ci tient au fait que l'homme est, par essence, un animal libre, même si, empiriquement, cette liberté demeure une « liberté dans la condition », autrement dit, non pas absolue, mais relative aux lieux et aux circonstances qui limitent les conditions de possibilité de son exercice.

Le signe de cette liberté est le phénomène de l'articulation, car il manifeste la capacité qu'ont les différentes parties du corps humain à se mouvoir de façon autonome, d' «après des concepts » (ou représentations), ce qui suppose la volonté. Ce signe ou symbole qu'est l'articulation permet à Fichte (1984, pp. 90 sq.), à la suite de Kant (1968 $\S 63$ à 68), de distinguer trois ordres du réel.

Le premier ordre est celui de la nature inorganisée, de la matière. Cet ordre est dominé par le mécanisme et il est l'objet de connaissance des sciences physico-chimiques. On n'y trouve, ni finalité, ni individualité à proprement parler.

Le deuxième ordre est celui de l'être organisé, dont le prototype est la plante ${ }^{3}$. L'organisme ne saurait être confondu avec la machine puisqu'il ne peut être compris sans le recours à la finalité. Cependant, dans l'ordre de l'organisation nous n'accédons pas encore à la sphère de l'individualité absolue mais seulement à celle de l'individualité relative dont le critère est la possibilité de la greffe. En effet, le fait de pouvoir greffer un arbre prouve que l'arbre n'existe pas vraiment comme individu puisqu'il tolère que vienne s'adjoindre à lui une greffe qui va en modifier la nature. Inversement, le fait que l'on puisse en détacher un rameau et le greffer sur un autre arbre prouve que le rameau détaché n'était pas une partie indissociable de l'arbre, et donc que celui-ci, dont on l'a détaché, n'est pas véritablement un « in-dividu », un tout qui est détruit comme tel si l'on en soustrait une partie. "C'est pourquoi, dit Kant, on peut considérer chaque rameau, chaque feuille d'un arbre comme simplement greffé ou écussonné à celui-ci, c'est-à-dire comme un arbre existant pour lui-même qui s'attache simplement à un autre arbre et se nourrit comme un parasite » (Kant, 1968, p. 191).

Le troisième ordre est celui de l'individualité absolue. Avec plus de précision que Kant, Fichte indique le critère empirique du vivant supérieur, du vivant animal : l'articulation et l'impossibilité de la greffe. C'est cette dernière qui nous fait passer de l'individualité relative (l'articulation) à l'individualité absolue (la vie animale). À ce niveau, l'individu en tant que vivant accompli, possédant un corps articulé qui lui permet d'agir selon des représentations, accède, dans le cas de l'homme, au rang de "personne », c'est-à-dire d'être responsable (au sens moral comme au sens intellectuel puisque les deux ordres renvoient à la notion de rationalité comme critère d'identification de la nature

\footnotetext{
${ }^{3}$ Cf. Kant, 1968, § 64, pp. 189-191, et Fichte, 1984, qui écrit pp. 93-94 : « Par l'hypothèse qu'un phénomène est un produit de la nature, on a un concept complet si tout ce qui se manifeste en lui, se rapporte à nouveau à l'organisation et se laisse expliquer complètement à partir de la fin de cette organisation déterminée. Par exemple la forme suprême et ultime, le degré extrême de la forme organisatrice dans les plantes considérées en leur individualité, c'est la semence : or celle-ci se laisse expliquer complètement par l'organisation en tant que fin; c'est par la semence que l'espèce se reproduit; c'est par elle que l'organisation à nouveau, retourne en elle-même et recommence son cours $(3,379)$ depuis le début. L'acte de cette force organisatrice n'est donc nullement achevé, mais se poursuit en un cycle éternel ».
} 
humaine) et libre, donc capable de communiquer directement et intentionnellement avec autrui en qui il reconnaît un alter ego.

On voit par conséquent qu'il reste à subdiviser le troisième ordre pour faire apparaître la sphère du vivant proprement humain à l'intérieur du vivant, ce qui sépare l'homme des animaux, mêmes supérieurs, dirions-nous aujourd'hui. En effet, la vie, dans ses formes les plus achevées, comprend à la fois l'humanité et l'animalité. Aussi, il convient de différencier le corps articulé et individualisé humain et le corps articulé et individualisé animal de telle manière qu'il soit possible, dès la manifestation empirique d'une certaine structure spécifique, de conclure à la présence d'un homme, d'un être à mon essence pareil. Autrement dit, le corps humain doit nécessairement être immédiatement saisi comme la présence sensible de la raison, et donc de la liberté. Selon Fichte (1984, p. 92), « Je ne puis comprendre le phénomène d'un corps humain si ce n'est en admettant qu'il est le corps d'un être raisonnable, signifie par conséquent: je ne puis m'arrêter, dans l'assemblage de ses parties avant d'être parvenu au point où je suis obligé de le penser comme corps d'un être raisonnable ».

Il s'agit dès lors de montrer comment le corps articulé de l'homme, à la différence du corps articulé de l'animal, impose la référence au concept de liberté pour être pensé adéquatement. Or s'il en est ainsi c'est parce que l'homme est le seul être qui «originairement n'est rien ». Cette mise en évidence de l'humanité de l'homme comme " phénoménologie de la liberté »" s'opère de la façon suivante.

Le corps humain doit être pensé comme un phénomène organisé. Il m'apparaît comme un tout et, dans sa perception, je ne suis pas sans cesse renvoyé à des éléments intrinsèquement hétérogènes, mais qui seraient agencés par un mécanicien extérieur de telle manière qu'ils coopèrent les uns avec les autres. Il est donc essentiel de ne pas confondre, comme l'avait déjà fait remarquer Kant, cette totalité organisée avec un « produit de l'art» ou artefact ${ }^{5}$. Ce qui est pensable comme un tout, c'est un "produit organisé de la nature », par exemple une plante ; et l'homme, comme la plante, est bien un tout dont les parties s'impliquent réciproquement. L'organisation ne suffit toutefois pas à expliquer le corps humain qui est plus qu'une plante parfaite dont la finalité, nous l'avons vu, est la semence à partir de laquelle l'espèce se reproduit.

L'articulation précisément échappe à la finalité reproductrice. Elle fait signe vers une autre fin qui est le mouvement libre. Cependant, ce mouvement non assujetti aux lois de la reproduction, tout corps articulé en est capable, qu'il soit animal ou humain ; aussi, pour distinguer le mouvement libre animal du mouvement libre humain, Fichte (1984, p. 95) introduit-il les concepts de détermination et d'indétermination. Si l'animal, comme l'homme agit selon des concepts ou représentations et possède ainsi un libre mouvement, celui-ci est déterminé par l'instinct et il n'exprime donc aucune réflexivité authentique et accomplie. On peut dès lors considérer que, s'il existe de la raison dans l'animal, elle lui reste étrangère. L'ordre est bien intériorisé mais il reste hétéronome. Chez l'homme seulement, il n'existe aucune déterminité de l'articulation mais une déterminabilité à l'infini ; aucune structure absolument contraignante, mais une structurabilité à l'infini.

\footnotetext{
${ }^{4}$ Nous reprenons ici l'interprétation que propose Alain Renaut (1986, pp. 213-215) de la pensée de Fichte.

${ }^{5}$ Cf. Fichte, 1984, p. 93 : « Toutefois, dans le produit de la nature, le tout existe aussi au service des parties : il n'a pas d'autre fin que celle de produire précisément ces parties ; dans le produit de l'art, en revanche, le tout ne renvoie pas aux parties, mais à une fin extérieure à lui : il est instrument pour autre chose ».
} 
Alors que tous les animaux sont fondamentalement complets et achevés, l'homme n'est qu'une ébauche, une esquisse, dont l'essence est l'éducabilité et la perfectibilité toujours inachevées. C'est la raison pour laquelle Fichte l'appelle «libre mouvement indéterminé ». Si l'individualité confère à l'animal une singularité qui le différencie de ses congénères, celle-ci est donnée une fois pour toute, alors que l'individualité de l'homme est aussi, et avant tout, l'effet du processus d'auto effectuation du moi qui est ce qu'il n'est pas et qui n'est pas ce qu'il est. Seul l'homme est véritablement confié à lui-même (et à ses semblables). C'est dans sa capacité à s'arracher à l'ordre des choses qu'il témoigne de son humanité et accède simultanément, de ce fait même, aux sphères de l'éthique et de la culture. ${ }^{6}$

\section{La position criticiste au regard de l'éthologie}

Parce qu'il est « néant », comme le dit Fichte, parce qu'il doit construire lui-même son identité, l'homme occupe donc une place particulière et privilégiée au sein du règne animal. Contrairement aux autres mammifères supérieurs, il ne possède pas d'environnement spécifique à son espèce, c'est-à-dire fermement structuré par sa propre organisation instinctuelle. Il n'existe pas de monde de l'homme au sens où l'on peut parler d'un monde du cheval ou du chien ${ }^{7}$. En dépit d'une zone d'apprentissages et d'accumulations individuels, tout cheval ou tout chien entretient avec son environnement une relation fortement déterminée qu'il partage avec les autres membres de son espèce. Chez les animaux, la relation à l'environnement est biologiquement fixée même si une variation géographique est introduite. De ce point de vue, comme individu ou comme espèce, l'animal vit dans un monde clos dont les structures sont prédéterminées par son équipement génétique. Par contraste, la relation de l'homme à son environnement est caractérisée par l'« ouverture au monde». L'homme a non seulement pu s'installer sur la plus grande partie de la planète, mais il a réussi à créer une relation à l'environnement qui n'est que très accessoirement structurée par sa constitution biologique.

Des travaux d'éthologie, à commencer par ceux de Karl Lorenz (1984, 1970), nous paraissent confirmer assez largement la justesse, en tout cas la solidité, de l'argumentation criticiste. La particularité de la constitution biologique de l'homme concerne sa composante instinctuelle ${ }^{8}$. Celle-ci peut être qualifiée de «sousdéveloppée » par rapport à celle des autres mammifères supérieurs. L'homme a bien sûr des «poussées », mais ces poussées sont essentiellement non spécialisées et non

\footnotetext{
${ }^{6}$ Fichte, 1984, p. 95 : «Chaque animal est ce qu'il est; l'homme seul, originairement n'est absolument rien. Ce qu'il doit être, il lui faut le devenir; et, étant donné qu'il doit en tout cas être un être pour soi, il lui faut le devenir par soi-même. La nature a achevé toutes ses œuvres, pour l'homme uniquement elle ne mit pas la main et c'est précisément ainsi qu'elle le confia à lui-même. La capacité d'être formé, comme telle, est le caractère propre de l'humanité (31). Par l'impossibilité de supposer pour une forme humaine un quelconque autre concept que celui de lui-même, tout homme est forcé intérieurement de tenir tout autre pour son semblable ». ${ }^{7}$ Cf. les travaux de von Uexküll, et notamment, « Mondes animaux et monde humain », 1965.

${ }^{8}$ Un comportement peut être qualifié d'«instinctuel» quand il présente les caractéristiques suivantes : être hérité au sens génétique ; être propre à une espèce ; varier faiblement d'un individu à un autre et se dérouler selon une séquence temporelle peu susceptible de bouleversement; atteindre d'emblée et sans apprentissage sa perfection ; paraître répondre à une finalité.
} 
dirigées. Autrement dit, l'organisme humain est capable d'appliquer l'outillage fourni par sa constitution à une gamme d'activités à la fois variable et changeante. Cette singularité réside dans son développement ontogénétique. En effet, l'organisme humain manifeste une très grande souplesse de réponse aux forces et sollicitations de son environnement interne et externe. Cela est particulièrement évident lorsqu'on observe la flexibilité biologique de l'homme soumise à diverses déterminations de nature socioculturelle. C'est pour cette raison que les manières de devenir et d'être un homme sont aussi nombreuses que les formes de sociétés humaines. En d'autres termes, il n'existe pas de nature humaine au sens d'un substrat, biologiquement fixé, qui déterminerait la variabilité des formations socioculturelles. Il n'existe de nature humaine qu'au sens de constantes anthropologiques (la souplesse de la structure instinctuelle, précisément, rendant possible l'" ouverture au monde ») qui délimitent et permettent les différents types d'expression culturelle des individus et des groupes. On peut donc considérer que l'homme construit sa nature proprement humaine, précisément parce qu'il est « libre mouvement indéterminé ».

Dans le comportement animal, au contraire, il faut admettre une sorte de " programme instinctif » qui se reconnaît là où, écrit Lorenz (1984, pp. 70-71), " un comportement, dont l'objectif nous apparaît clairement, fait également apparaître un décalage entre les facultés d'intelligence que nous avons généralement observées chez l'animal et celles qu'il aurait fallu pour mener l'enchaînement en question à son terme volontairement [...] et là où le déroulement du comportement présente des failles qui montrent incontestablement que l'animal n'a pas lui-même conscience de l'objectif de son action. ». Un grand nombre de comportements semblent en effet réglés selon des schémas qui présentent des formes temporelles très complexes, des sortes de "mélodies d'impulsion » et qu'il faut interpréter comme des mouvements instinctifs ou " coordinations héréditaires » représentant en fait le «squelette du comportement d'une espèce donnée » (Lorenz, 1984, pp. 66-67).

$\mathrm{Au}$ sein de cette « unité fonctionnelle », Lorenz distingue deux mécanismes physiologiques différents. Tout d'abord un «mécanisme inné de déclenchement» (Mid) qui donne à l'animal une « connaissance innée » de la situation environnementale biologiquement adéquate pour l'emploi du comportement en question. Ensuite, une « coordination motrice héréditaire » programmée phylogénétiquement qui, une fois mise en route par le mécanisme de déclenchement, maîtrise cette situation avec une connaissance innée (Lorenz, 1984, p. 198).

Le fait remarquable constitutif du Mid est l'existence de clés dans l'environnement. «Des combinaisons déterminées d'excitations agissent souvent comme des clés en déclenchant de manière très spécifique des réactions déterminées ; ces mêmes réactions peuvent ne pas être déclenchées par des combinaisons d'excitations très semblables. Il existe donc une corrélation qui, un peu comme une serrure complexe, ne répond qu'à des combinaisons déterminées d'excitations et déclenche l'acte instinctif» (Lorenz, 1970, p. 209). Cela signifie que dans le Mid l'organisme ne réagit pas à une image complète de la situation environnementale adéquate, mais à une configuration de stimuli bien précise qui caractérise schématiquement cette situation. « Il ne s'agit donc pas comme chez l'homme d'une réaction à la forme complexe de l'objet naturel (qui ferait l'objet d'une perception authentique), mais à des configurations de stimuli clés qui doivent s'enchaîner d'une manière précise, un peu comme une mélodie, où ce qui 
joue un rôle déterminant, ce ne sont pas les valeurs absolues mais les intervalles de tons » (Lorenz, 1984, pp. 197-198). C'est pour cette raison qu'il est possible de leurrer l'animal et par là même d'étudier par variations la séquence de configurations de stimuli qui opère le déclenchement de son comportement. En effet, l'information fournie par le Mid est trop simple et trop peu précise et ne porte que sur des valeurs relatives, donnant ainsi la possibilité d'exagérer les caractères jouant le rôle de stimuli clés et de fabriquer des leurres que l'animal préférera à l'objet naturel adéquat (Lorenz, 1984, pp. 200-220). On voit ainsi en quoi on peut parler pour l'animal d'une sorte de «capture comportementale » relative qui fait de son action un «libre mouvement déterminé » au sens de Fichte.

Il faut encore ajouter, pour montrer le caractère « déterminé » mais non mécanique du comportement animal, que celui-ci manifeste véritablement de l'«appétit» pour l'acte instinctif qu'il cherche à exécuter (Lorenz, 1970, pp. 237-238), ce qui conduit au double constat suivant: tout d'abord, l'animal est bien le «sujet» d'une véritable appétence, sorte de prolégomènes du désir, mais pour des comportements instinctifs vers lesquels il est poussé ; ensuite, son «appétence » est assouvie, non par la rencontre des stimuli déclencheurs, qui peuvent fort bien manquer, mais par l'accomplissement de l'acte lui-même. Le comportement de l'oie cendrée décrit par Lorenz le montre clairement: « $\mathrm{Si}$ au retour d'un long vol, elle se pose maladroitement dans une haie dont elle ne peut repartir qu'en volant, elle a certes une assez grande compréhension de la situation pour essayer d'atteindre, par des mouvements de secouement du bec et des mouvements d'envol, une accumulation d'excitation suffisante pour s'envoler, mais elle peut mettre très longtemps pour y parvenir. Un stimulus extérieur supplémentaire peut provoquer la motivation nécessaire, mais en dépit de sa compréhension, l'animal n'a pas la possibilité de se donner une impulsion que nous appellerions chez l'homme une "décision" ». Cette "appétence » qui s'extériorise de manière désordonnée et agitée, nous dit Lorenz, consiste plutôt à subir la poussée dans ce qu'elle a d'impératif, quitte à ce que, parvenue à un niveau suffisant, celle-ci déclenche le mouvement «à vide».

Le champ d'intellection de l'animal est donc étroitement limité, et son investigation ne porte pas vraiment sur la clé qui va déclencher son mouvement instinctif, mais, avant que la poussée n'arrive à ce seuil où le comportement s'accomplirait à vide, il porte sur cet élément de l'environnement ou sur le signal déclencheur lui-même, inné en tant qu'il fait partie intégrante de l'acte instinctif. Bref, il semble que le comportement instinctif qui se cherche dans l'« appétence » ne se sait pas comme tel et ne peut donc constituer, comme chez l'homme, l'objet spécifique d'un désir susceptible d'une confirmation langagière ${ }^{9}$.

À l'objection selon laquelle les conclusions de Lorenz ne sont peut-être pas valides pour les animaux supérieurs, et notamment les singes, la consultation de recherches récentes de primatologues permet d'apporter une réponse qui ne remet pas fondamentalement en cause les thèses du chercheur allemand. Nous prendrons l'exemple de la célèbre

\footnotetext{
${ }_{9}^{9}$ On peut considérer que dans la « jouissance fonctionnelle », dont parle Lorenz (1984, p. 389), il y a quelque chose qui manifeste la liberté animale dans ce qu'on peut appeler une « ouverture au monde » pré-langagière et préconsciente. Celle-ci est confirmée par le comportement de curiosité des animaux supérieurs, qui «ne peut être activé par aucune autre motivation et ne peut l'être que tant que tous les autres se taisent ».
} 
synthèse sur la culture des chimpanzés publiée dans la revue Nature par un groupe d'éthologues (Whiten et al., 1999, pp. 682-685).

Sur la base de nombreuses observations indépendantes conduites dans la longue durée sur des populations de chimpanzés éloignées les unes des autres, les auteurs montrent que des groupes distincts élaborent des familles de techniques bien différenciées. Ce type de variations ne pouvant, semble-il, être expliqué par une évolution adaptative des comportements aux contraintes écologiques, les auteurs sont amenés logiquement à attribuer aux chimpanzés des cultures distinctives, c'est-à-dire une liberté d'inventer des réponses sui generis aux nécessités de la subsistance et de la vie commune.

Cependant, on fera remarquer que pour invalider la théorie criticiste et plus largement la philosophie humaniste, il faudrait mettre en évidence que les singes sont aptes à produire de la singularité culturelle en mobilisant des facultés internes qui leurs sont propres, autrement dit, qu'ils ont une «théorie de l'esprit », pour reprendre l'expression de Joëlle Proust (2000). Il faudrait montrer qu'ils font appel à des ressources psychiques identiques aux nôtres lorsqu'ils s'engagent dans des activités culturelles. Or ce point reste obscur. Les éthologues constatent des variations dans les systèmes techniques des animaux sauvages et décrivent minutieusement des procédures au moyen desquelles ils se diffusent d'individu à individu, mais ils demeurent assez vagues concernant les conditions mentales et neurophysiologiques nécessaires pour ce faire. Ils évoquent seulement une aptitude générale à l'imitation qui pourrait dériver, chez les grands singes, du contrôle de l'attention d'autrui, et qui laisse perplexes beaucoup de psychologues expérimentalistes. Ces derniers, attachés à comparer l'apprentissage social chez les enfants humains et chez les chimpanzés captifs récusent l'idée même que ces animaux soient capables d'imiter. Ils considèrent que les enfants apprenant à utiliser un outil ont une représentation du but poursuivi par leur instructeur, tandis que les chimpanzés se contentent de régler leurs comportements les uns sur les autres par ajustements successifs au cours d'occurrences répétées suscitant l'émulation (Tomasello et Call, 1997).

Le débat est donc vif entre les éthologues de terrain enclins à prêter aux animaux qu'ils observent des propriétés mentales susceptibles de rendre compte de leurs actions, et les éthologues de laboratoire qui affirment ne pas trouver trace de ces hypothétiques propriétés chez les animaux qu'ils étudient. Mais en fait, étudient-ils véritablement les mêmes animaux ? Montrer que le comportement des chimpanzés sauvages est affecté par des variations culturelles, parce que certaines de leurs facultés cognitives seraient identiques aux nôtres, impose en effet qu'on leur fasse effectuer des tâches en laboratoire qui permettent la comparaison systématique avec les humains. Or, les éthologues font justement remarquer que le contexte du laboratoire est si particulier, en raison de l'interaction entre les animaux captifs et les expérimentateurs, que les résultats obtenus ne peuvent guère être généralisés aux primates sauvages.

À propos de la question du langage, on constate également que, majoritairement, les éthologues cognitifs ne sont pas disposés à attribuer aux animaux une véritable pensée consciente, considérant que le langage animal est le produit d'une prédisposition psychique encodée dans le cerveau, et dont les caractéristiques varient en fonction du génome de leur espèce. Le langage animal procéderait ainsi d'une détermination neurophysiologique peu à peu spécifiée par l'expérience, sans qu'il soit nécessaire pour l'expliquer de faire intervenir la médiation de représentations complexes, c'est-à-dire d'aptitudes propositionnelles 
grâce auxquelles l'animal objectiverait ses propres états internes (émotions, croyances, intentions) et interpréterait ceux des autres animaux présents dans son environnement (Marler, 1991, pp. 37-66).

Plus globalement, concernant les relations entre culture et apprentissage et le fait que les primatologues de terrain observent l'existence de savoir-faire propres à une communauté d'animaux relatifs à la préparation de la nourriture, Joëlle Proust (2000, pp. 15-17) propose une interprétation qui récuse également tout rapprochement décisif avec le modèle humain. «Par exemple, les macaques japonais de Koshima ont appris en quatre ans à laver leurs patates douces pour les débarrasser de la terre après qu'un seul individu eut inventé cette technique. Mais ce cas relève-t-il de l'enseignement, pratique intentionnelle dirigée vers autrui? Rien dans la technique des macaques ayant acquis la technique de nettoyage n'indique le moindre souci pédagogique. La lenteur de l'acquisition tend à montrer que les animaux n'acquièrent l'innovation ni par enseignement délibéré, ni même par imitation. Là où l'observateur non averti croit voir le fruit d'un enseignement, l'éthologue repère un phénomène d'“intensification de stimulus": le simple voisinage spatial entre un membre du groupe et l'objet-cible élève l'intérêt des congénères pour ce type d'objet et suscite chez eux des tentatives d'utilisation ».

Toujours selon Joëlle Proust (2000, p. 16), il en est de même pour l'hypothèse du mensonge chez les primates : «Après avoir, pendant un temps, conclu que les primates non humains peuvent mentir, c'est-à-dire se conduire délibérément de manière à produire chez autrui des croyances fausses, les chercheurs admettent aujourd'hui que ces comportements peuvent s'expliquer par apprentissage des comportements efficaces dans une situation donnée. Il n'est pas nécessaire, pour produire les résultats attendus, de savoir que les congénères agissent sur la base de leurs croyances. Il suffit simplement de découvrir le type d'action à faire ou à ne pas faire dans telle ou telle circonstance [...]. L'hypothèse de l'existence d'une théorie de l'esprit chez l'animal ne se soutient plus aujourd'hui que d'anecdotes rassemblées par les propriétaires de grands primates domestiques ».

\section{L'imagination créatrice comme dimension spécifique de l'humanité : rupture et indétermination}

Résumons-nous. Il faut penser le développement de l'organisme humain comme inséparable de celui du moi et selon des modalités qui vont donner lieu à des productions socioculturelles que l'on ne retrouve pas dans les espèces animales, ou alors seulement peut-être à l'état d'ébauche. La relation du moi à son organisme se présente ainsi comme « excentrée ». D'une part, l'homme est un corps, ce qui est également vrai de tout organisme animal ; mais, d'autre part - et c'est là son originalité - il $a$ un corps. Cela signifie que l'homme fait l'expérience de lui-même comme d'une entité, certes inséparable de son corps, mais non identique à celui-ci et qui, de surcroît, peut disposer (au moins en partie) de ce corps. En d'autres termes, son expérience de lui-même est toujours en équilibre problématique entre l'être et l'avoir. C'est pour cette raison que l'activité humaine doit toujours être interprétée à la fois comme intégrée dans un environnement matériel et comme extériorisation d'intentions subjectives. 
Par ailleurs, l'humanité psychique de l'homme et sa socialité sont empiriquement mêlées de façon inextricable. C'est toujours collectivement (y compris dans la lutte) que les hommes construisent un environnement humain. La dynamique de l'ordre social n'est pas biologiquement donnée. Ce qui est biologiquement donné, c'est l'instabilité originaire de son organisme, qui oblige l'homme à créer « artificiellement », autrement dit, culturellement, l'environnement stable de ses propres conduites. Bien qu'aucun ordre social existant ne puisse être issu d'un déterminisme biologique, la nécessité de l'ordre social en tant que tel résulte de l'équipement biologique spécifique de l'homme qui le contraint en quelque sorte à l'autonomie et à l'imagination créatrice.

Pour prendre toute la mesure de ce que Cornélius Castoriadis appelle « l'imaginaire radical » (1975), il faut concevoir celui-ci comme création première et considérer que la distinction apparemment fondatrice opérée par l'ontologie traditionnelle entre le réel et l'imaginaire n'est en fait qu'une opposition dérivée de cette imagination première (Castoriadis, 1975, pp. 7-8). Il existe dans tout être vivant en tant qu'il est «pour soi », précise Castoriadis, une imagination élémentaire. En effet, il doit se poser comme « autofinalité », ce qui implique une intentionnalité minimale, celle de se conserver en puisant dans le monde extérieur les moyens de sa reproduction. Il constitue ainsi son monde propre à partir d'un environnement initialement étranger, car il faut bien insister sur le fait que le vivant ne se contente pas d'accueillir des informations qui existeraient en soi dans le monde extérieur. C'est bien plutôt l'être vivant lui-même qui crée en quelque sorte les informations avec lesquelles il constitue son «chez soi ». L'imagination et la logique à l'œuvre dans le vivant sont, dès le départ, fixées de manière rigide et constituent un système rigoureusement fonctionnel qui vise l'autoconservation de l'espèce. Ce n'est qu'avec l'apparition de l'homme que se manifeste une rupture dans l'ordre du " pour-soi », puisque l'être humain se singularise par le caractère fondamentalement «a-fonctionnel» de son imagination. Alors que le «pour-soi » de l'animal ne peut être déconnecté de sa constitution neurosensorielle, le psychisme humain est caractérisé par la domination du plaisir représentatif sur le plaisir organique, la séparation de la sexualité humaine d'avec la reproduction constituant l'une de ses conséquences les plus remarquables. Cette a-fonctionnalité se manifeste chez l'homme, par le remplacement, en tout cas la surdétermination de la satisfaction biologique par la satisfaction « hallucinatoire », qui présuppose non pas tant la capacité à voir des images ou à se voir en tant qu'image dans un miroir, mais bien plutôt l'aptitude à poser ce qui n'est pas, ou plus exactement à voir dans quelque chose ce qui n'y est pas. C'est cela qui permet, par exemple, au nourrisson de produire la représentation du sein absent sous forme de fantasme et d'en jouir sur un mode hallucinatoire, ou encore, à un enfant plus âgé, d'imaginer un chien dans les cinq lettres du mot ${ }^{10}$.

\footnotetext{
${ }^{10}$ Bien entendu, la radicalité de l'imaginaire humain ne lui permet pas pour autant de passer outre certaines exigences de la vie biologique et certaines obligations sociales. Hormis les contraintes imposées par la matérialité du monde et les exigences de la vie qui font que l'homme peut connaître et utiliser les lois de la nature mais pas les transgresser, il faut insister sur le fait que l'imaginaire crée de la nouveauté à partir des mondes culturels déjà institués. Il est donc pris dans l'historicité du social. Les modèles et normes dont hérite une collectivité ne la prédestinent pas, mais ils la pré-orientent, lui ouvrent des voies et lui en ferment d'autres, au moins pour un certain temps. Par ailleurs, si son institution imaginaire n'est pas rationnelle au sens instrumental et stratégique du terme, si elle n'est pas un projet pleinement conscient, explicitement réfléchi et parfaitement maîtrisé dans sa conduite, car elle est aussi constituée d'affects et de désirs, elle ne peut cependant être viable
} 
Bref, les représentations de l'imaginaire radical ne constituent pas la reproduction - fût-elle idéale - d'une réalité première. « Il n'y a aucune possibilité de comprendre la problématique de la représentation si on cherche l'origine de la représentation hors de la représentation elle-même. La psyché est certes "réceptivité des impressions", capacité d'être affectée-par ; mais elle est aussi (et surtout - sans quoi cette réceptivité des impressions ne donnerait rien) émergence de la représentation en tant que mode d'être irréductible et unique et organisation de quelque chose dans et par sa figuration, sa "mise en image". La psyché est un formant qui n'est que dans et par ce qu'il forme et comme ce qu'il forme ; [...] elle est imagination radicale qui fait surgir déjà une "première" représentation à partir d'un rien de représentation, c'est-à-dire à partir de rien » (Castoriadis, 1975 p. 383).

L'imaginaire ne peut cependant être réduit à la seule dimension de la psyché, qui est, par sa nature même, a-sociale et par conséquent inapte à la vie. Ainsi, un nourrisson qui en resterait au stade du seul plaisir hallucinatoire deviendrait rapidement psychotique. D'où la nécessité de socialiser la psyché, afin de lui faire accepter la présence d'autrui, non pas seulement comme limite, mais comme condition positive de la réalisation de son désir. C'est aux institutions qu'incombe la charge de fabriquer, à partir de la psyché, un individu social à part entière. Mais si la psyché des individus humains ne peut exister que socialisée, il reste que dans son fond elle ne l'est jamais totalement, et ce fond d'être présocial constitue toujours une menace pour les institutions, en même temps qu'une condition de possibilité du changement.

Faisons le point. De tous les êtres vivants, l'homme n'est pas le seul à vivre en société ; l'ordre animal connaît aussi des formes complexes de vie en collective. Ce que l'homme apporte de spécifique à la vie en société s'appelle la «culture» et, si celle-ci répond bien à la nécessité de s'organiser en commun pour survivre, les formes historiques qu'elle va prendre sont largement indéterminées parce qu'elles sont l'effet de sa libre praxis et des systèmes symboliques (langage, mythes, religions, sciences...) qu'elle met en œuvre. La différence entre l'homme et l'animal réside précisément dans la nécessité où le premier se trouve pour assurer sa survie individuelle de passer par une médiation collective d'essence culturelle.

La caractéristique propre du domaine culturel par rapport au domaine naturel est non seulement sa plus grande complexité mais aussi et surtout son degré plus élevé d'indétermination. Dans le registre naturel, nous l'avons vu, les mécanismes biologiques instinctuels déterminent le comportement animal de manière immédiate et spontanée. La connaissance des éléments d'une situation donnée nous permet de prédire de manière quasi certaine le comportement caractéristique de la réaction animale. En revanche, dans le registre socioculturel, l'immédiateté et la spontanéité du déterminisme naturel sont nettement moins affirmées. Les possibilités de solution étant beaucoup plus élastiques et arbitraires, le degré de prévisibilité des comportements individuels et collectifs diminue considérablement.

On comprend ainsi pourquoi les sociétés animales sont relativement immuables. En effet, dans celles-ci, l'individu étant exclusivement identifié à ses fonctions répondant

\footnotetext{
.../... sans présenter une configuration logique, une cohérence structurale, parce que la rationalité est une composante de l'essence du réel. L'être n'est pas un désordre absolu, sa consistance « ensembliste-identitaire » ou « ensidique », pour parler comme Castoriadis, fait de l'usage de la raison une condition de possibilité de toute vie sociale.
} 
aux exigences de la survie du groupe, il ne peut qu'agir selon une logique de maximisation de son identité sociale. Dans les sociétés humaines, au contraire, la part d'indétermination introduite par la réflexivité et sa dimension d'autonomie rompt l'immédiateté et la spontanéité de la reproduction fonctionnelle ${ }^{11}$. Il ne s'agit pas de nier le soubassement biologique du comportement humain, car il est vrai que la référence aux problèmes d'ordre vital dans leur rapport aux conditions matérielles de la survie permet toujours d'établir un certain degré de prévisibilité des actions humaines. L'interprétation de celles-ci est cependant rendue plus complexe par le nombre quasi infini des solutions symboliques au moyen desquelles les sujets individuels et collectifs cherchent à justifier et consolider leurs problèmes d'identité et d'appartenance selon une logique existentielle qui peut aller à l'encontre même des exigences de la vie biologique ${ }^{12}$.

Certes, pour qu'il y ait unité sociale il faut bien que les actions des individus soient prévisibles en partie et donc conformes à des normes préconstituées; mais celles-ci, loin d'être imposées par la nature, sont nées du choix initial de ceux qui ont réussi à les imposer à l'ensemble de la collectivité. La culture, en fournissant des modèles et des règles de comportement, correspond à l'exigence essentielle de fonder la prévisibilité sociale. Elle cherche ainsi à rétablir un automatisme analogue à celui du régime instinctuel, mais sans jamais y parvenir complètement, à cause précisément de son caractère de médiation, de distanciation par rapport à la vie immédiate, qui laisse toujours la possibilité, même infime, d'autres options comportementales.

L'écart est donc constitutif de la réflexivité. S'il permet de rompre avec le mécanisme instinctuel, il empêche également sa reproduction au plan symbolique autrement que de manière analogique et à titre provisoire. D'un côté, les impératifs de l'action, aussi bien que l'angoisse suscitée par le risque de l'imprévu, poussent la conscience à s'attacher à une forme particulière de détermination culturelle et à l'absolutiser. L'ordre symbolique retenu fournit ainsi un stock de prévisions et de certitudes nécessaires à la stabilité sociale. D’un autre côté, parce qu'elle est le lieu de l'écart et de la différence, la conscience, quoique fixée dans une forme symbolique déterminée, en perçoit aussi (même confusément) les limites et les entraves. De là son refus des normes qui pourraient l'enfermer et sa recherche incessante de formes nouvelles de médiation symboliques et sa tendance à se déplacer indéfiniment d'une forme à l'autre. Ce n'est pourtant qu'en acceptant à la

\footnotetext{
${ }^{11}$ Cf. Crespi (1983, p. 12) : «L'homme est le seul animal normalement capable de désordre, c'est-à-dire d'actualisation de comportements irréductibles au modèle fonctionnel du système vivant, au point de s'opposer aux règles de la survie de l'individu et du groupe. Même les dimensions instinctuelles qui sont présentes dans l'homme (instinct de conservation, instinct de reproduction, etc.), restent subsumées dans la modalité existentielle particulière qui s'ouvre avec la conscience et ne peuvent être simplement rapportées à une composante purement biologique ».

${ }^{12}$ Cf. Rousseau, (1969, p. 47) : «Je ne vois dans tout animal qu'une machine ingénieuse, à qui la nature a donné des sens pour se remonter elle-même, et pour se garantir, jusqu'à un certain point, de tout ce qui tend à la détruire ou à la déranger. J'aperçois précisément les mêmes choses dans les machines humaines ; avec cette différence que la nature fait tout dans les opérations de la bête, au lieu que l'homme concourt aux siennes en qualité d'agent libre. L'une choisit ou rejette par instinct, et l'autre par un acte de liberté ; ce qui fait que La bête ne peut s'écarter de la règle qui lui est prescrite, même quand il lui seroit avantageux de le faire, et que l'homme s'en écarte souvent à son préjudice. C'est ainsi qu'un pigeon mourroit de faim près d'un bassin rempli des meilleures viandes, et un chat sur des tas de fruits et de grains, quoique l'un ou l'autre pût très bien se nourrir de l'aliment qu'il dédaigne, s'il s'étoit aviser d'en essayer. C'est ainsi que les hommes dissolus se livrent à des excès qui leurs causent la fièvre et la mort parce que l'esprit déprave les sens, et que la volonté parle encore quand la nature se tait ».
} 
fois le déterminé, c'est-à-dire la nécessité d'un ordre symbolique, et l'indéterminé, c'està-dire la limite de cette médiation symbolique, que l'on peut comprendre que le monde social humain est la concrétisation des projets de la conscience intentionnelle comme lieu de la différence et analyser à partir de là les liens entre liberté et contrainte. Si le sujet n'est pas seulement l'expression de sa détermination biologique ou sociale, mais aussi celle de sa capacité à reconnaître et à dépasser les limites de cette détermination, il est donc en même temps le lieu d'origine de la définition des normes et le lieu de leur infraction. La dynamique tensionnelle entre ces deux pôles opposés et complémentaires définit alors la liberté comme capacité du sujet à gérer, dans ses interactions avec d'autres sujets, le rapport déterminité-indéterminité au sein même de l'organisation sociale qui constitue la base et l'horizon de son expérience spécifique. N'est-ce pas là le propre de l'homme ? ${ }^{13}$

\footnotetext{
${ }^{13}$ On notera au passage que, selon les défenseurs de la «libération animale », tel Peter Singer, l'animal est respectable comme une personne morale pourvue d'une dignité intrinsèque fondée sur l'éthique utilitariste qui fait de la capacité à éprouver du plaisir ou de la peine le critère de la dignité d'un être et le constitue comme personne juridique. Au contraire, pour la pensée humaniste, c'est la liberté, ou capacité à s'arracher aux intérêts immédiats, qui définit la dignité suprême et fait du seul être humain une authentique personne juridique. La raison ou le langage ne sont donc pas des critères discriminants suffisants pour identifier la frontière entre l'humanité et l'animalité. Il est bien clair, cependant, que, si l'animal n'est pas assimilable à une personne, il ne saurait pour autant être traité comme un objet ou un artefact, et qu'il doit par conséquent bénéficier de tous les égards réservés à un être susceptible de souffrir.
} 



\title{
Chapitre 8
}

\section{La construction des mondes animaux et du monde humain selon Jacob von Uexküll}

\author{
FLORENCE BURGAT
}

Il est bien des manières de s'interroger sur ce qui distingue l'homme et les animaux, mais cette opération est le plus souvent conduite à des fins de hiérarchisation morale. Les animaux sont en effet examinés à l'aune de l'humain, et c'est de leur ressemblance avec lui que dépend leur statut moral, et donc la façon dont nous devons les traiter. Mon propos ne relèvera pas d'une telle orientation, tant il me semble important de s'émanciper du référent humain si l'on veut tenter de cerner ce que la vie animale, sous ses multiples formes, a de propre. C'est dans leur singularité aussi que les animaux doivent être regardés; à défaut, ils demeureront au mieux ces «frères inférieurs » au langage qui n'en est pas un, au monde qui n'est qu'un environnement, à l'intelligence qu'il vaut mieux nommer « instinct», etc.

Les auteurs qui sont ici convoqués ont magistralement contribué à cet effort de décentrement, élaborant, grâce à une approche phénoménologique, une conception de la vie animale pour elle-même et non comme prétexte à réitérer le propre de l'homme. Nous examinerons la «question des frontières entre l'homme et les animaux » à partir du travail de Jacob von Uexküll sur la manière dont est construit le monde propre à chaque espèce animale. On verra combien y est centrale la notion de signification. C'est aussi à une refonte du concept de comportement, loin de ses fondements béhavioristes, que l'on est conduit. Nous nous pencherons plus particulièrement sur les points suivants : qu'entend Jacob von Uexküll lorsqu'il qualifie l'animal de sujet dans son monde ? Pour ce qui concerne la construction des mondes animaux, comment le couple perception et action forme-t-il ce que Uexküll nomme « le cercle fonctionnel »? Peut-on dégager une structure d'ensemble qui, en quelque façon, subsumerait tous ces mondes ? L'harmonie $\mathrm{du}$ point et du contrepoint constitue le plan de la nature au sein duquel ceux-ci s'organisent. Il s'agit enfin d'en finir avec la double illusion d'un monde unique, d'une part, d'un monde humain en surplomb, d'autre part (le monde humain n'est en effet pas la 
somme des mondes animaux). Nous compléterons cet exposé par la perspective que Maurice Merleau-Ponty propose dans son ouvrage de 1942, La structure du comportement, et dans ses cours prononcés au Collège de France une quinzaine d'années plus tard, au fil desquels il commente la pensée de Uexküll ; le comportement y est en effet pensé comme relation dialectique au monde.

\section{L'animal : un sujet dans son monde?}

Jacob von Uexküll est un zoologiste spécialisé dans la physiologie musculaire. C'est au prix d'une immense audace théorique qu'il s'éloigne de l'étroitesse des vues de sa spécialité, pour attacher son nom à l'étude du monde propre à chaque espèce animale. Il se fait écologue, et dédie sa Théorie de la signification ${ }^{1}$ « à la bienveillante attention de [ses] adversaires scientifiques ». Disposition requise, puisque d'entrée de jeu la notion de sujet est introduite par Uexküll pour caractériser l'animal dans son monde, car l'animal ne saurait se penser hors de son monde. Qualifier l'animal de sujet, qu'est-ce à dire ? Il nous faut prendre des distances avec l'acception classique du concept de sujet - du sujet cartésien notamment, qui pense parce qu'il se sait penser. Il convient d'envisager ici un sujet sans conscience réflexive ; le champ ouvert par le concept de sujet est du reste plus large que celui embrassé par la conscience. Est en effet pour Uexküll un sujet tout individu qui agit et perçoit, et pour lequel s'ouvrent par conséquent un monde de la perception et un monde de l'action. Frederik Buytendijk n'hésite pas lui non plus à qualifier les animaux de sujets, et sa lecture de Uexküll n'est pas étrangère à cette audace. Doit être à ses yeux qualifié de sujet celui dont le «mode d'existence [qui] s'affirme comme le fondement d'une réceptivité aux significations intelligibles et en même temps d'une activité qui crée ces significations et y répond intelligemment» (Buytendijk, 1965, p. 22). On voit toute l'importance qu'il accorde, à la suite de Uexküll, à la signification dans le monde vivant capable de comportements. Car, pour Frederik Buytendijk, tout organisme n'est pas un sujet (même si tout organisme - plante ou cellule - constitue une unité de signification) et tous les mouvements ne sont pas des comportements; seuls le sont les mouvements spontanés, en tant qu'ils révèlent une situation. Le sujet existe, et ne fait pas que vivre, selon une opposition traditionnelle, bien simplificatrice, pour laquelle il y a la vie purement biologique (qu'est-ce à dire ?) de l'animal ou du végétal (dont rien ne permet de distinguer les modalités d'être) et l'exister humain. L'existence n'est en effet pas entendue par Buytendijk au sens, qu'il juge étroit, que lui confèrent tant la perspective anthropologique que les ontologies existentialistes. L'existence désigne chez lui « une situation manifestée par un comportement» (Buytendijk, 1965, p. 46). L'identité de structuration qu'il repère chez l'homme et chez l'animal - c'est-à-dire le fait que tous deux se constituent dans le comportement que, du même coup, ils façonnent - forme ce qu'il appelle la subjectivité.

Comme Uexküll l'annonce en avant-propos de ses analyses (2004), la difficulté inhérente à l'étude des mondes animaux tient non seulement au fait que ces mondes (ou milieux) nous sont à la fois inconnus et invisibles, mais encore au fait que leur existence même est contestée par la plupart des zoologistes, et plus encore par les physiologistes.

\footnotetext{
${ }^{1}$ J. von Uexküll, 2004, Mondes animaux et monde humain (désormais noté MAMH) suivi de Théorie de la signification (désormais noté TS).
} 
Tout biologiste digne de ce nom et soucieux de se démarquer du physiologiste doit, selon Uexküll, adosser ses recherches au principe selon lequel l'être vivant est un «sujet qui vit dans son monde propre dont il forme le centre » (2004, « Mondes... », p. 19), et non l'objet qu'y voit le physiologiste. La décision de Uexküll d'étudier le monde spécifique de l'animal s'inscrit clairement dans une perspective critique de la biologie d'inspiration mécaniste. Celle-ci voit en effet dans les êtres vivants des machines et assimile l'organisme à un complexe d'outils. Lorsque Uexküll déclare que « c'est la signification qui est le fil directeur sur lequel la biologie doit se guider, et non la misérable règle de causalité qui ne peut voir plus loin qu'un pas en avant ou un pas en arrière, et reste aveugle aux grandes relations structurelles » (2004, «Théorie... », p. 117), il exprime le décentrement qu'il opère au regard de la biologie mécaniste.

Parce que l'animal est un sujet, il se trouve au centre de la construction du milieu (ou du monde propre) : " ce sont les actions des animaux projetées dans leur milieu qui confèrent leur signification aux images perceptives grâce à la connotation d'activité » (Uexküll, 2004, «Mondes... », p. 60). De manière générale, le monde propre de l'animal se découpe de la façon suivante : les objets saillants d'un milieu sont ceux qui ont une connotation d'activité pour l'animal. Plus les actions possibles sont diversifiées et nombreuses, plus le monde est riche, mais combien plus périlleux. La représentation picturale que Uexküll donne d'une même pièce d'habitation humaine vue par l'homme, le chien et la mouche montre quels sont les objets chaque fois chargés ou non d'une connotation d'activité : le bureau permet à l'homme d'écrire (cet objet renvoie donc pour l'homme à cette fonction), tandis qu'il constitue pour le chien qui se couche en-dessous une sorte d'abri, etc. Avec les mammifères, et Uexküll (2004, p. 80) observe surtout le chien familier, des «élaborations subjectives » sont à l'origine d'une délimitation du territoire qui ne répond plus à la seule activité : certains lieux attirent le chien sans que l'observateur puisse comprendre les raisons de leur pouvoir attractif. Ce sont, dit Uexküll, des « lieux magiques » $(2004$, p. 80), dont le sujet est seul à percevoir l'intérêt particulier, un intérêt qui s'attache à des critères autres qu'objectifs. Avec le territoire et la maison émergent des qualités qui ne regardent pas d'abord les registres de la causalité et de la finalité ; en d'autres termes, ce serait pour d'autres types de raisons que les animaux choisiraient leurs lieux. Puisse ce mystère demeurer celé...

\section{Perception et action : le cercle fonctionnel}

Comment les signaux perceptifs deviennent-ils les caractères des objets extérieurs ? Comment un objet fait-il sens pour moi ? Pour parler de manière imagée, l'objet saillant pour le sujet animal est comme enserré dans les deux branches d'une pince, dit Uexküll, dont l'une correspond à la dimension perceptive de l'objet et l'autre à sa dimension active. L'action de l'animal est conditionnée par le choix d'excitations que laissent passer les récepteurs, comme par l'agencement des muscles qui rend telle action possible et non telle autre. Cela forme ce que Uexküll nomme le cercle fonctionnel; ce cercle est ce qui relie le sujet aux objets qui sont ceux de son monde.

C'est avec l'exemple de « la tique et son milieu » que Uexküll choisit d'ouvrir son analyse du monde animal. L'étroitesse du monde de la tique permet d'expliciter la manière dont les objets acquièrent une signification pour un sujet - car la tique est un sujet au sens défini plus haut : elle perçoit et agit. Ce qu'il s'agit de comprendre, c'est comment et 
pourquoi, lorsqu'un mammifère passe à portée de la tique, seuls trois effets (l'acide butyrique, le toucher, la chaleur) provenant du corps du mammifère sont pour elle porteurs de caractères perceptifs. Les trois stimulants qu'elle perçoit comme significatifs de proie sont dotés de cette signification parce qu'ils rendent possible la triple activité de se laisser tomber, d'explorer et de perforer. Ni le biais explicatif mécaniste de l'excitation chimique de l'acide butyrique, ni l'effet, mécanique, provoqué par les poils du mammifère, ni celui, thermique, de sa peau, ne peuvent selon Uexküll rendre compte de ce qui se passe entre la tique et le mammifère. À s'en tenir au schéma stimulus-réponse, on manque la question du monde, et donc du sujet, c'est-à-dire de sa relation avec certains objets et pas d'autres. Cette conversion des stimulants en signaux est possible parce que la tique est pourvue de trois signaux perceptifs qu'elle peut transformer en caractères perceptifs ; et son monde propre se réduit aux trois caractères évoqués.

Il est des animaux, comme l'oursin ou la méduse, dont le cercle fonctionnel se limite à un seul élément. Tel est le cas de la méduse, dont tout l'organisme se borne à n'être qu'une pompe flottante qui absorbe l'eau de mer riche de plancton. Pour ce qui concerne les animaux dont le monde se ramène à un seul cercle fonctionnel, on peut parler, dit Uexküll, d' " animal-réflexe ». La tique, malgré l'étroitesse de son monde limité à trois cercles fonctionnels, représente un type d'animal supérieur à la méduse ou à l'oursin, car les cercles fonctionnels sont en elle rattachés à un organe de perception commun et non à des arcs réflexes isolés. Cet aspect conduit Uexküll à faire l'hypothèse que pour la tique, le mammifère-proie qu'elle identifie forme une unité. Cela ne doit cependant pas donner à penser que la tique guette sa proie : elle n'est pas un sujet au sens où ses actions seraient guidées par une intention; elle ne dispose du reste point des outils psychophysiologiques nécessaires à une telle visée.

Comment expliquer qu'un objet soit porteur de signification pour un individu d'une espèce donnée et non pour un individu d'une autre espèce ? On l'a dit, la perception et l'action sont au fondement de la connotation que prend un objet. On peut faire l'expérience de mettre différents animaux en face d'un objet pour savoir quels sont ceux qui entreront en relation avec cet objet. Expériences mille fois conduites et qui n'ont, souligne Uexküll, abouti à rien de convainquant, du fait qu'étrangers à la théorie des milieux, les observateurs sont partis du présupposé faux qu'un animal peut entrer en relation avec un objet comme tel. Or chaque objet est porteur d'une signification spécifique, et c'est par le sujet que la signification advient à l'objet. L'armoire dans laquelle sont rangées des couvertures n'a pas la même signification pour l'être humain que pour le chat qui aime à s'y installer douillettement et y dormir. Cela vaut aussi bien à l'intérieur du monde humain où les objets ne sont pas absolument tous porteurs des mêmes significations. Il n'y aurait pas non plus pour l'homme d'objet comme tel, le « comme tel » exprimant la possibilité de le détacher de tout contexte utilitaire. On peut prendre l'exemple simple d'un objet fabriqué que je vois pour la première fois et dont la finalité d'usage ne m'apparaît pas. L'idée d'une signification unique de l'objet s'efface. Des connotations différentes peuvent s'attacher à un même objet : je peux voir dans la canne qui accompagne de longues marches un objet contondant, dans la tasse un vase possible, dans un outil dont l'usage est évident à son artisan une forme mystérieuse... Pour le dire autrement, la même image perceptive peut être chargée de plusieurs images actives. On peut détourner un objet de la signification première à laquelle répond son usage le plus courant, c'est-à-dire celui pour lequel il a été façonné. Il en va de même dans le monde 
naturel où la tige d'une fleur des champs constitue un objet d'émerveillement pour l'enfant, un chemin pour la fourmi, un aliment pour la vache. En revanche, plus large est le monde du sujet, plus grande la possibilité de faire varier la signification des objets. En effet, le chien dressé à sauter sur une chaise au commandement « chaise », note Uexküll, sautera sur un autre objet pouvant faire office de chaise (des caisses ou des étagères renversées). Cela montre que la connotation d'un objet dépend des actions qu'il permet à un sujet d'accomplir.

Ce point est très important, car il indique que le monde du mammifère peut s'enrichir de bien des objets qui à première vue lui sont indifférents, au sens où ils ne sont porteurs d'aucune signification. Cela est patent dans le cas des animaux familiers qui habitent dans la maison de l'homme. Si un autre objet que celui initialement destiné à une action satisfait aux critères de réalisation de cette action, il sera vu comme un même porteur de signification. Parce que le chien est doté d'une structure nerveuse complexe, il est capable, comme le montre cet exemple, d'identifier une même connotation dans des objets différents. Cette possibilité de variation est synonyme de liberté par rapport aux choses.

Il existe donc une certaine ambiguïté des objets. Buytendijk se démarque à nouveau de Uexküll lorsqu'il n'attribue de monde ambigu qu'à l'homme, du fait de la capacité de recul qu'il peut prendre à l'égard de toute situation (le « comme tel»). Ce serait parce que l'homme peut s'extraire de son environnement, qu'il peut faire varier la signification de ses objets. Buytendijk reconnaît cependant aux animaux supérieurs vivant au contact de l'homme la capacité à apprendre l'ambiguïté, c'est-à-dire à voir une chose sous deux aspects différents ; mais cela constitue une exception à la règle si, pour lui, l'ambiguïté est un propre de l'homme. Le chien, pourtant, se distingue des autres animaux par le fait qu'il cherche « quelque chose ». Outre son incroyable aptitude à entrer dans le monde humain (à en acquérir les habitudes, à percevoir l'intention des actes de l'homme), le chien poursuit un but, et non point seulement une trace. Cela signifie qu'il use de détours pour atteindre « quelque chose », ce qui met par ailleurs en évidence la position vers « le lointain spatio-temporel » (Uexküll, 2004, p. 127) qui est la sienne.

On résumera la pensée de Uexküll en disant que « chaque action, avec sa composante perceptive et active, imprime sa signification à tout objet neutre et en fait dans chaque milieu un porteur de signification rattaché au sujet» (2004, p. 100).

\section{Le plan de la nature : point et contrepoint}

C'est à un «plan de la nature» (notion qui vaut probablement à Uexküll d'être parfois classé parmi les néo-vitalistes) que tout être vivant est subordonné. Mais, dans la considération de ce plan, il faut commencer par se débarrasser d'un double fourvoiement : la finalité, d'une part; le mécanisme d'autre part, qui n'ont que trop marqué la pensée biologique. Le plan de la nature, auquel Uexküll recourt pour rendre compte de l'essence des relations qui unissent le sujet aux objets qui s'offrent à lui sous la figure de porteurs de signification, n'est aucunement superposable à un quelconque finalisme de la nature. Ce plan de la nature fait plutôt figure de transcendance ordonnatrice ou de structure. Lorsque les individus agissent en fonction d'un but, comme cela est le cas pour l'homme et au moins pour tous les mammifères, leurs actions n'en sont pas moins subordonnées au plan général de la nature. Celle-ci constitue « la totalité qui transcende 
chaque milieu particulier » (2004, p. 90) et sans laquelle nous serions face au chaos des perspectives propres à chaque espèce.

Approfondissons maintenant la question de savoir comment un objet devient signifiant pour un individu au regard du plan de la nature. Il s'agit de comprendre comment, au niveau biologique, l'organisme actualise les significations que les porteurs de signification lui offrent. Le mystère de la toile d'araignée est cette fois l'exemple qui illustre ce propos. Ce mystère réside dans le fait que la toile met en œuvre la signification « proie », sans que l'araignée n'ait au préalable rencontré la mouche à partir de laquelle elle eût pu élaborer son piège. Aussi, Uexküll nous invite-t-il à regarder la toile comme la copie fidèle de la mouche. Tendue dans les endroits où passent les mouches, elle est par ailleurs tissée de sorte que l'œil de la mouche soit incapable de la distinguer.

Cette harmonie préétablie entre le sujet et son objet nous donne à voir une nature qui a par avance ordonné la relation signifiante unissant un sujet à ses objets. C'est en cela que tient le plan de la nature. Du point de vue biologique, cela signifie ici que le «pour la mouche» de la toile d'araignée, c'est-à-dire le fait que celle-ci soit fondamentalement destinée à capturer des mouches, est intégré dans le corps de l'araignée elle-même. La croissance embryonnaire répond à un plan préétabli (dit « plan de signification englobant »), au sens où la partition présente dans le développement embryonnaire trouve son prolongement dans le monde sensoriel. «Je prétends, écrit Uexküll, que la partition originelle de la mouche [...] agit de telle sorte sur la partition originelle de l'araignée que la toile que tisse cette dernière peut être qualifiée de "mouchière" " (2004, p. 117). Autre exemple de ce type de correspondance : pourquoi le papillon de nuit ne perçoit-il que le cri de la chauve-souris ? Il y a là encore la marque d'un accord préétabli, dont les structures corporelles de la chauve-souris et du papillon se font l'écho. La règle du développement embryonnaire inscrit biologiquement l'ouverture de telle espèce à telle signification. Et les choses se passent probablement selon un ordre au sein duquel c'est la règle de signification qui agit sur la règle de développement - et non l'inverse. La correspondance réciproque qui lie le porteur de signification à ce qui le met en œuvre trouve, avec la règle du développement embryonnaire, son explication biologique.

Le modèle de la composition musicale aide à comprendre la structure contrapuntique qui sous-tend le monde sensoriel dans lequel les objets sont pourvus de signification. Le système de correspondance entre deux notes, qui forme une harmonie, est comparable à ce qui unit dans la nature le sujet pris dans son milieu et les objets qu'il perçoit comme étant porteurs de signification pour lui. Tout, dit Uexküll, obéit dans la nature à un ordre contrapuntique : à chaque point son contrepoint. Ainsi en est-il de la feuille de chêne (récepteur de signification) et de la pluie (facteur de signification). Tout organe sensoriel est adapté à ce qu'il doit percevoir. Aussi l'hirondelle et la chauve-souris ne disposentelles pas des mêmes organes pour appréhender les obstacles qui peuvent se dresser devant chacune d'elles. La mouche forme le contrepoint pour la toile d'araignée, qui est le point. Ainsi, « rien n'est laissé au hasard dans la nature », constate Uexküll, " partout une règle de signification très intime relie l'animal à son environnement physique et les réunit en un duo dans lequel les particularités des deux partenaires composent un contrepoint » (2004, p. 134). Nos objets usuels eux-mêmes, note Uexküll, sont fabriqués selon cette structure : à l'anse de la tasse revient le rôle contrapuntique pour le point qu'est la main humaine qui la saisit. 


\section{La double illusion d'un monde unique et d'un monde humain en surplomb}

L'illusion d'un monde unique s'effondre dès lors que l'on envisage la diversité des mondes animaux. On a une idée du chaos que forme l'ensemble des mondes propres à chaque espèce lorsqu'on s'applique à décrire la même chose vue dans différents milieux, et donc par des individus n'appartenant pas à la même espèce. Uexküll prend l'exemple du chêne qui, habité par de nombreux animaux, joue aussi un rôle dans plusieurs milieux humains. Le forestier portera sur l'arbre un regard évaluateur à l'occasion de son plan de coupe, tandis que l'enfant, pour qui la forêt est le lieu fantastique des contes, s'effrayera du visage grimaçant que composent les rides de son écorce. Le tronc du chêne offre une tanière au renard, ses branches un refuge à la chouette, etc. Cette variation sur un même objet aboutit à la destruction de son unité : chaque groupe animal ou humain représenté ici prend en vue une certaine partie du chêne en fonction de son activité. De chêne en soi, pourrait-on dire, il n'y a point : " Si l'on voulait rassembler tous les caractères contradictoires que présente le chêne en tant qu'objet, on n'aboutirait qu'à un chaos »; pourtant, le chêne existe bel et bien comme unité qui contient en elle tous les milieux évoqués, mais il existe « sans être reconnu ni jamais pouvoir l'être par tous les sujets de ces milieux » (2004, p. 88). C'est donc en parvenant à isoler les caractères perceptifs retenus par l'animal parmi la multitude de caractères que comporte l'immensité de son entourage, que l'on verra se dessiner le contour du monde propre de cet animal. Aussi est-il erroné de croire qu'il existe un monde unique dans lequel prendraient place les êtres vivants. Chaque espèce découpe son milieu ou son monde propre, c'est-à-dire un ensemble d'objets et une forme spatio-temporelle qui n'appartiennent qu'à elle.

Le monde de l'homme est-il ce monde qui se tiendrait en surplomb des mondes animaux ? Le monde humain comprend-il la diversité des mondes animaux ? L'homme est-il celui qui, au contraire de tous les animaux, peut s'extirper du milieu qui est le sien ? Pour Uexküll, nul ne peut s'extirper de son milieu, pas même l'homme, qui demeure lié à sa nature d'homme et donc à un milieu propre à celle-ci. L'homme a certes fabriqué des instruments qui lui permettent de mieux connaître son milieu et de l'élargir géographiquement, mais « il n'est pas d'instrument qui permette de sortir du milieu » (2004, p. 162). Le monde humain n'est aucunement celui qui coiffe ou surplombe les mondes animaux; le point de vue de l'homme n'est pas non plus la somme de tous les points de vue animaux.

Sous l'influence de Max Scheler, Buytendijk abandonne Uexküll sur ce point. Il refuse en effet l'idée que l'homme posséderait un monde environnant ne différant pas, dans sa structure, de celui des animaux. Et l'exemple du chêne qui sert à Uexküll à étayer cet aspect apparaît illégitime à Buytendijk : la comparaison des regards du forestier, du poète, de l'enfant, du hibou et de l'écureuil est à ses yeux erronée, au motif que «l'homme n'a pas un environnement, mais un monde » (Buytendijk, 1965, p. 56). Il veut dire par là que le monde de l'homme n'est pas uniquement constitué par des objets apparaissant spécifiques, mais essentiellement par le fait que l'homme fait face à un ensemble qui est le monde. Cela signifie que l'homme peut à la fois en être partie prenante et spectateur, pris dans une situation et dans le même temps, simultanément, porter sur elle un regard désintéressé. 
Buytendijk reconnaît cependant que plus on s'élève dans la complexité, plus on est conduit à s'interroger sur le monde des animaux supérieurs; celui du chien qui vit avec l'homme et dont le monde est chargé d'une certaine ambiguïté, mais surtout celui de l'anthropoïde à propos duquel il se demande si son monde environnant ne doit pas être envisagé « comme une forme primitive du monde humain $»^{2}$. La distance spatio-temporelle complique en effet la relation au milieu, et force à prendre en compte dans l'analyse du comportement des animaux supérieurs le décalage obligé qui structure le mouvement spontané visant un but, parfois lointain, dans l'espace et dans le temps. Buytendijk, malgré ces réserves, demeure fidèle à l'idée que l'animal, « espèce naturelle » ne saurait prendre véritablement de la distance à l'égard de la situation; et que la liberté lui manque donc (Buytendijk, 1965, p. 69).

\section{Le comportement comme relation dialectique au monde}

Maurice Merleau-Ponty trouve dans l'œuvre de Uexküll une matière qui nourrit et prolonge ses propres analyses du comportement animal. Pour lui, cet entourage est une ouverture et non une fermeture. À cette orientation ne souscrivent ni Buytendijk ni Max Scheler, qui opposent le monde et le milieu. Ce dernier écrit en effet «L'homme est donc l' $x$ qui peut, sans limites aucune, se comporter comme un être "ouvert au monde". L'animal, lui, n'a pas d'“objets"; il vit seulement plongé extatiquement dans son milieu que, tel un escargot, sa coquille, il apporte comme structure partout où il va. Il est donc incapable de ce recul spécial et de cette substantification qui d'un "milieu" font un "monde" [...]» (Scheler, 1951, p. 56). Comment caractériser le mode de relation par lequel un individu entre en rapport avec ce qui l'entoure ? Qu'est-ce qu'un comportement? Forgé dans la mouvance réductionniste du béhaviorisme watsonien, le concept de comportement fut à l'origine l'affaire du seul corps, et d'un corps compris comme un pur organisme réagissant à des stimuli. Le statut du stimulus, et de la théorie du réflexe qui l'accompagne, est au centre de la critique élaborée dans La Structure du comportement (Merleau-Ponty, 1977), où Maurice Merleau-Ponty s'efforce de faire apparaître l'inanité des réponses apportées par la psychologie de laboratoire au problème du comportement. Peut-on traiter les faits de comportement sur le modèle de ceux de la nature ? Telle est la question posée par la phénoménologie au béhaviorisme qui les porte sur le plan de la causalité physique.

Il revient à Maurice Merleau-Ponty, puis à Frederik Buytendijk, d'avoir élaboré, contre l'approche béhavioriste, une conception du comportement comme relation dialectique avec l'entourage pour le premier, comme relation intelligible avec ce qui est donné, manifestation d'un «être en situation » pour le second (Buytendijk, 1965, p. 13) ; se comporter, c'est « être quelque part, avec et dans ». La relation ainsi appréhendée avec l'entourage est bien de nature dialectique. Elle inclut par conséquent une relation dynamique à l'espace comme au temps : le comportement est en lui-même une activité en devenir, ou encore « un débat en cours avec une situation » (Buytendijk, 1965, p. 67). Ces deux conceptions se tiennent dans une très grande proximité théorique, et Buytendijk se réfère du reste explicitement aux travaux de Merleau-Ponty.

\footnotetext{
${ }^{2}$ Buytendijk, 1965, p. 57. Dans son Traité de psychologie animale, 1952, Buytendijk évoque longuement le cas de la femelle chimpanzé Viki, et du chimpanzé en général.
} 
C'est sur la spécificité de l'objet étudié (le comportement) que leur critique des approches béhavioristes porte; et c'est en particulier lorsqu'on se trouve face aux comportements supérieurs que l'impasse dans laquelle nous conduit le schéma stimulusréponse apparaît dans toute sa lumière. En effet, comment expliquer que l'organisme entre en relation avec un milieu infiniment plus étendu et plus riche que celui qui agit immédiatement, sous forme de stimulations physiques et chimiques, sur ses déterminations sensorielles ? Comment passer outre le " caractère vécu » de toute " situation corporelle », qui fait irrémédiablement de toute « existence physique », une « existence confuse » (Thinès, 1980, p. 27) ? N'est-il pas remarquable qu'à des conditions extérieures identiques répondent des comportements intra-spécifiques différents ? Comment, à partir de la théorie des réflexes, rendre compte d'un comportement nouveau et pourtant adapté ? Voilà ce que la compréhension béhavioriste du comportement ne saurait élucider. Merleau-Ponty ne craint pas de voir dans cette indétermination fondamentale du comportement un "commencement de culture » : quand on se tourne vers les comportements animaux les plus complexes, on voit les animaux accéder au plan symbolique, et «l'architecture des symboles, que l'animal apporte de son côté, définit ainsi, au sein de la nature une espèce de pré-culture » (Merleau-Ponty, 1995 p. 231). Aussi l'Umwelt des animaux supérieurs est-il de moins en moins découpé par un but, pour l'être par des symboles. Faisant valoir les réponses globales de l'organisme et la «structure indécomposable des comportements » (Merleau-Ponty, 1977 p. 48), Merleau-Ponty défend, sur la base d'une approche entièrement refondue de la notion de comportement, l'idée d'un «certain milieu caractéristique de l'espèce » (1977 p. 136) - idée déjà formulée par Uexküll3 ${ }^{3}$. Distinct de l'entourage géographique, le milieu propre à chaque espèce est défini par Merleau-Ponty comme une réalité intermédiaire entre le monde tel qu'il pourrait exister pour un observateur absolu (un point de vue de surplomb) et un point de vue purement subjectif. Le monde auquel l'animal s'adresse n'est autre que celui pour lequel son comportement existe ou, pour le dire cette fois dans les termes de Uexküll, celui dans lequel des objets font sens pour lui.

Ce qui explique encore l'intérêt que trouve Merleau-Ponty à la lecture de Uexküll est l'introduction d'un sujet sans subjectivité, sauf à entendre ici la subjectivité comme l'activité perceptive-active. Car Merleau-Ponty insiste sur le fait que le comportement n'a pas besoin d'une conscience pour être, et c'est ce qui lui permet de penser, hors du dualisme qui ne le permet pas, le propre de l'être-au-monde de l'animal. Le comportement - telle est sa spécificité - n'aurait sa place ni dans l'ordre de l'en-soi ni dans celui du pour-soi. «Dans l'expérience des comportements, note Merleau-Ponty, je dépasse effectivement l'alternative de l'en soi et du pour soi [...] Refuser aux animaux la conscience pure, la cogitatio, ce n'est pas faire d'eux des automates sans intérieur. L'animal [...] est bien une autre existence» (Merleau-Ponty, 1977, p. 137). En d'autres termes, il s'agit de passer du corps comme «objet de la physiologie mécaniste » à la «synthèse du corps propre », pour reprendre les titres de deux des sections de la Phénoménologie de la perception (Merleau-Ponty, 1976). L'activité comportementale

\footnotetext{
${ }^{3}$ Uexküll, 2004, MAMH. Notons que cet ouvrage n'est pas mentionné dans La Structure du comportement, mais qu'il sera largement cité dans La Nature..., aux côtés d'un autre ouvrage de Uexküll : Umwelt und Innenwelt des Tiere, 1909, dans lequel il rejetait déjà la théorie selon laquelle les activités animales résultent de causes physico-chimiques, au profit d'une analyse des relations de l'individu avec son monde environnant.
} 
existe bien avant l'invention de la conscience, souligne Merleau-Ponty, dans la mesure où on peut parler de comportement " dès que l'on a des stimulations qui agissent, non par simple présence physique, mais en tant qu'un organisme est disposé à les recevoir et à les traiter comme des signaux » (Merleau-Ponty, 1995, p. 220). Tout comportement est sous-tendu par une dimension projective : elle est ce mouvement par lequel l'organisme manifeste une possibilité qui lui est intérieure, marquant ainsi de ses empreintes le plein du monde, creusant des trous au cœur de la positivité de la matière physique. En d'autres termes, le comportement, en tant qu'il est libre, introduit du néant dans le monde.

La conscience n'est pour Merleau-Ponty que l'une des formes variées du comportement, lequel embrasse à la fois l'embryologie, l'organisation physiologique, l'instinct et le comportement proprement dit. Le comportement est lisible dès les premiers stades du développement embryonnaire, si l'idée d'une référence à l'avenir est contenue dans l'embryon dès avant la formation du système nerveux. L'enracinement biologique du comportement dans l'organisme conduit à penser ce dernier comme le «synonyme de comportements possibles dans l'avenir» (Merleau-Ponty, 1995 p. 198). Les organes n'ont en effet de sens qu'en référence à un futur. L'organisme n'est pas seulement un centre téléphonique; il faut, pour le comprendre, inclure «l'inventeur et l'opérateur du téléphone » (1995 p. 194). Le comportement doit donc être regardé comme un principe immanent à l'organisme ; il est une totalité émergente. Merleau-Ponty compare la structure du comportement à celle du langage : tout comme lui, il est un système de différences où chaque mot, chaque posture, ne prend sens qu'à l'égard des autres mots ou des autres postures. Il n'est par ailleurs ni une chose, ni une idée - et c'est ce qui le rend opaque pour l'intelligence. Le comportement est qualifié par Merleau-Ponty de « forme », notion qui a le mérite d'intégrer les trois plans du physique (système de forces dans lequel le comportement prend place), du physiologique (dont il faut reconnaître le caractère original) et du mental (comportement symbolique). Penser le comportement comme une forme nous permet, selon Merleau-Ponty, de sortir des apories auxquelles le matérialisme comme le vitalisme conduisent : ce n'est ni la mystérieuse force vitale ni la conception de l'organisme pensé comme une machine, fût-elle doublée d'une conscience, qui peuvent rendre compte de l'intelligibilité du comportement.

\section{Conclusion}

La forme que propose Merleau-Ponty d'une part, la théorie contrapuntique qui décrit la relation qui unit l'organe aux significations du monde d'autre part, invitent, dans une perspective refondue de la biologie, à penser le comportement comme un rapport dialectique, et donc jamais donné d'avance - ce sont en effet des « relations toujours nouvelles » (Merleau-Ponty, 1977, p. 161) qui sont produites - entre l'animal dont l'« histoire est toujours vide de ce qui va suivre » (Merleau-Ponty, 1995, p. 207) et son milieu propre. Avec le comportement animal, des relations inédites traversent le monde pour y déposer ses marques, « si bien qu'on ne voit plus bien où commence le comportement et où finit l'esprit » (1995, p. 233). 


\title{
Chapitre 9
}

\section{La morale au royaume des animaux : rechercher une éthique animale, est-ce naturaliser la morale ?}

\author{
CATHERINE LARRÈRE, RAPHAËL LARRÈRE
}

\section{Introduction}

Dans la philosophie occidentale, l'affirmation de l'existence d'une morale dans le règne animal relève principalement d'une tradition sceptique. C'est ainsi, par exemple, que Montaigne a transmis un recueil d'anecdotes antiques (Plutarque, Pline, etc.) : les hommes n'ont pas à se glorifier de leur moralité, car les Anciens ont fort justement observé que les animaux se conduisent bien mieux qu'eux.

Dans le contexte contemporain, la question de la moralité animale est celle de l'observation de comportements que l'on peut qualifier de moraux. Elle fut introduite par Darwin (1999), aux chapitres 4 et 5 de La filiation de l'homme: comment l'évolution a-t-elle sélectionné les comportements sociaux, ou altruistes, que l'on repère chez certains animaux ? Darwin s'est alors intéressé à la genèse des sentiments moraux, principalement de la sympathie, influencé qu'il était par David Hume et Adam Smith. Conjointement, il s'interrogeait sur l'émergence d'une « identification» de l'individu à sa «communauté » telle qu'elle peut le conduire à lui sacrifier ses pulsions ou sa vie.

La question est reprise dans l'éthologie contemporaine de deux façons distinctes :

- la sociobiologie en fait son problème central. Selon Edward O. Wilson (1987), il s'agit bien de savoir comment l'altruisme a pu se développer par l'intermédiaire de la sélection naturelle : la réponse est alors à rechercher dans la génétique des populations : si l'altruisme de certains individus leur permet d'avoir une postérité plus féconde que les autres, et si ce comportement est héréditaire, alors les gènes qui prédisposent à l'altruisme se répandront dans le pool génétique de leur espèce ; 
- la primatologie (et particulièrement l'étude des chimpanzés) s'intéresse aux comportements moraux mais aussi à ce que l'on peut reconstituer de la motivation, des intentions, des émotions, des sentiments, des connaissances des animaux qui se comportent de la sorte.

L'idée générale qui se dégage de ces démarches est que la morale n'est pas spécifique à l'homme. Celui-ci a hérité de comportements moraux qui ont émergé et se sont répandus chez les animaux sociaux au cours de l'évolution : nous sommes présélectionnés comme des individus adoptant des comportements altruistes, avant d'être capables de déterminer rationnellement nos choix moraux.

Cela ne signifie pas que les comportements ainsi caractérisés soient ensuite proposés comme des exemples ou des règles morales. L'éthologie évolutionniste se distingue aussi bien du darwinisme social à la Spencer que des leçons que Pierre Kropotkine (1979, p. 30) avait tiré de sa lecture de Darwin :

« Il apparaît (...) que non seulement la nature ne nous donne pas de leçon d'amoralisme, c'està-dire d'indifférence à l'égard de la morale, indifférence qu'un principe étranger à la nature devrait combattre pour la dominer, mais, au contraire, que nos notions du bien et du mal (...) sont empruntées à la vie de cette même nature ».

Avec la sociobiologie et la primatologie contemporaines on ne se trouve pas confrontés à un " sophisme naturaliste » dérivant ce qui doit être de ce qui est. Cela ne signifie pas néanmoins, que la description de comportements moraux dans le règne animal n'ait pas d'implication en philosophie morale.

Cette façon d'aller rechercher l'émergence de comportements moraux et de les analyser chez des animaux est généralement comprise comme une "naturalisation » de la morale. Elle l'est au sens le plus immédiat: les animaux faisant partie de la nature, montrer que certains d'entre eux agissent moralement, c'est trouver une morale naturelle. C'est ainsi que Frans De Waal a intitulé l'un de ses ouvrages : Le bon singe: les bases naturelles de la morale. Cette naturalisation a aussi un sens plus technique, celui de la sociobiologie, qui s'inscrit dans un programme de "naturalisation » de toutes les facultés cognitives et de tous les comportements. Mais il est vrai que cette émergence de comportements altruistes à partir d'une soupe primitive de comportements égoïstes résulte en sociobiologie de la simulation sur ordinateur des interactions qui s'établissent entre des comportements représentés par des algorithmes. Si bien que cette «naturalisation de la morale » est en fait une " artificialisation de la morale » : ce qui rend compte de l'existence d'altruisme chez les animaux, comme chez les humains, est formellement établi par l'évolution d'une population d'automates.

Nous voudrions ici remettre en cause cette assimilation de l'observation des comportements moraux des animaux à une naturalisation de la morale, et ce dans les deux domaines où cette étude a été menée. Nous procéderons d'abord à une présentation critique de la sociobiologie: le programme de naturalisation de l'altruisme passe à côté de l'animalité. Nous verrons ensuite que l'on ne peut rendre compte de la moralité des primates qu'en renonçant à les considérer comme des animaux purement naturels (déterminés par la génétique et l'environnement) : il convient de prendre en compte une dimension des sociétés de primates que l'on peut qualifier de « culturelle». 


\section{Altruisme et théorie des jeux - présentation critique de la sociobiologie}

La démarche sociobiologique s'inscrit ouvertement dans une tradition darwinienne. Elle se conforte ainsi de deux présupposés :

- si l'on repère, chez des espèces animales des comportements que l'on pourrait qualifier « d'altruistes » (les soins accordés aux descendants, les secours aux congénères, le sacrifice d'individus au bénéfice de la communauté), c'est qu'il y a dans le génome de ces animaux une information génétique favorisant cet « altruisme »;

- si de tels comportements «altruistes » sont stables et généralisés, c'est qu'ils ont été sélectionnés : ceux qui possédaient les gènes prédisposant à «l'altruisme » ont eu une descendance plus prospère que ceux qui ne les possédaient pas. Cet avantage compétitif dans la lutte pour assurer sa descendance a donc permis aux gènes de « l'altruisme » de se répandre.

\section{Hamilton et l'altruisme de parentèle}

Pour rendre compte des soins accordés par les parents (oiseaux et mammifères) à leurs petits, ou bien pour expliquer la raison d'être du sacrifice des insectes sociaux stériles, qui travaillent pour les reproducteurs de la ruche ou de la fourmilière, sans se reproduire eux-mêmes, Darwin avait émis l'hypothèse d'une sélection familiale (sans en préciser les modalités). Hamilton (1964, pp. 1-5) va reformuler la question en considérant que la sélection s'opère au niveau du génome des individus et non de celui de la famille ou du groupe social. Tout organisme tend à se reproduire et, de ce point de vue, l'objectif est la postérité de ses gènes. Cette postérité, que Hamilton désigne par fitness (capacité de dissémination des gènes d'un individu dans sa descendance), est le mécanisme par lequel certains caractères, parce qu'ils sont adaptés au contexte écologique, se répandent dans une espèce donnée. L'argument de Hamilton est que, sous certaines conditions, un individu assurera mieux la dissémination de ses gènes en aidant un individu apparenté à se reproduire (quitte à diminuer sa propre descendance), que s'il consacrait toute son énergie à se reproduire ${ }^{1}$.

C'est ce mécanisme de sélection que Hamilton désigne par « altruisme de parentèle », et il voit dans les insectes sociaux la confirmation de sa théorie. Ces insectes sont, pour la plupart, des hyménoptères. Or les hyménoptères sont haplodiploïdes : les femelles

\footnotetext{
${ }^{1}$ Supposons qu'un individu A mobilise égoïstement toute son énergie pour survivre et se reproduire. Il aura une fitness propre de Fa. Supposons maintenant qu'il manifeste un comportement « altruiste » envers B, c'est-à-dire qu'il consacre une partie de son énergie à aider $\mathrm{B}$ à augmenter sa propre fitness (qui passe de $\mathrm{Fb}$ à $\alpha \mathrm{Fb}$ ), et ce, au détriment de la sienne, qui devient $\gamma \mathrm{Fa}$. Puisque $\gamma \mathrm{Fa}<\mathrm{Fa}$, alors que $\alpha \mathrm{Fb}>\mathrm{Fb}$, le comportement altruiste de $\mathrm{A}$ devrait conduire son génome à avoir une postérité plus faible, sauf dans le cas où $\mathrm{A}$ et $\mathrm{B}$ sont apparentés et possèdent des gènes en commun (soit Pab le \% de gènes en commun de $\mathrm{A}$ et de $\mathrm{B}$ ). Dans ce cas, en effet, la fitness globale des gènes de $\mathrm{A}$ est : $\mathrm{FGa}=\gamma \mathrm{Fa}+\alpha \mathrm{Fb}(\mathrm{Pab})$. Cette fitness globale peut être supérieure à celle qu'aurait obtenu A s'il avait été égoïste. Il suffit que le coût de son altruisme (Fa $-\gamma \mathrm{Fa})$ soit compensé par l'augmentation de la postérité des gènes qu'il a en commun avec B. Il faut donc que : Fa $(1-\gamma)<\alpha \mathrm{Fb}$ $(\mathrm{Pab})$. Dans ces cas, la postérité des gènes de A est mieux assurée que s'il avait eu un comportement égoïste. Hamilton en conclut que l'altruisme aura d'autant plus de chance d'être sélectionné que l'aide apportée à B est plus efficace (ce qui suppose $\alpha$ plus grand) et que A et B ont un fort $\%$ de gènes en commun (Pab).
} 
sont diploïdes (elles possèdent 2 n chromosomes) alors que les mâles, issus d'ovules non fécondés, sont haploïdes (ils n'ont que n chromosomes). De ce fait, les sœurs d'une même mère ont en commun les trois quart de leurs gènes ${ }^{2}$. Ainsi, en investissant toute son énergie à aider sa mère à produire un grand nombre de sœurs, la femelle stérile (spécialisée dans la défense ou l'approvisionnement de la communauté) assure mieux la propagation de ses gènes chez les hyménoptères, que si elle s'accouplait pour produire des filles qui ne partageraient avec elle que la moitié de ses gènes.

Le modèle de Hamilton a eu d'autant plus de succès, que l'on a trouvé des formes de société semblables (avec sacrifice reproductif des filles au bénéfice de la mère) parmi des espèces d'insectes qui, tout en étant éloignées phylogénétiquement des hyménoptères, sont haplodiploïdes : certains acariens, certains scolytes et des trips (Jaisson, 1993). Pourtant Hamilton lui-même reconnaît que son modèle ne saurait expliquer les comportements sociaux des insectes qui, comme les termites, sont diploïdes (la distinction des sexes se faisant selon que l'individu possède la paire de chromosomes XX ou XY).

La démarche suivie par Hamilton a été développée, systématisée et appliquée à de nombreux caractères physiologiques ou comportementaux par Richard Dawkins (1978). Selon lui, la sélection naturelle ne s'opère ni au niveau de la population, ni au niveau des individus, mais au niveau du gêne. S'il est une entité que l'on peut qualifier d'égoïste, c'est bien le gène. Il ne peut que se dupliquer et, s'il n'assure pas la plus large propagation de ses copies, un gène quelconque finira toujours par disparaître. Aussi, pour Dawkins, l'altruisme (et pas uniquement l'altruisme de parentèle) « serait finalement le mécanisme le plus efficace que les gènes auraient développé au cours de l'évolution pour manipuler les organismes afin de s'assurer une propagation optimale » (cité par Jaisson, 1993, p. 60). Bien plus, les organismes eux-mêmes seraient les « artifices inventés par les gènes » pour se reproduire et se répandre : « il ne reste que des gènes qui se bagarrent sans arrêt sur le long terme et des organismes qui sont le produit de ce conflit perpétuel » (Gouyon, 2001, p. 48).

\section{Robert Trivers et l'altruisme de réciprocité}

L'objectif de Robert Trivers (1971, pp. 35-67) est de rendre compte de l'existence de comportements qualifiés d'altruistes entre individus d'une même espèce, même quand on ne peut soupçonner d'affinité génétique particulière entre eux. L'altruisme est défini comme un acte qui a un coût pour celui qui l'accomplit et un bénéfice pour celui qui en est le destinataire. Un tel comportement peut être sélectionné s'il est suivi d'un acte réciproque. Supposons un prédateur $\mathrm{P}$, face auquel une proie $\mathrm{X}_{1} \mathrm{n}$ 'a qu'une chance sur deux de sauver sa vie. Supposons qu'une autre proie potentielle $\mathrm{X}_{2}$ lui porte assistance et qu'à elles deux $\left(\mathrm{X}_{1}\right.$ et $\left.\mathrm{X}_{2}\right)$ elles aient neuf chances sur dix de sortir indemnes de leur confrontation avec $\mathrm{P}$. $\mathrm{X}_{2}$ n'a aucun intérêt à mettre sa vie en danger (même si le risque est relativement faible, puisqu'il n'intervient que dans un cas sur dix) pour porter secours

\footnotetext{
${ }^{2}$ Les filles stériles ont $2 \mathrm{n}$ chromosomes dont $\mathrm{n}$ (100\% des gènes paternels) viennent du père et $\mathrm{n}$ (soit $50 \%$ des gènes maternels) de la mère. Ces sœurs partagent donc $75 \%$ de leurs gènes alors que si elles se reproduisaient leurs filles n'auraient que $50 \%$ de leurs gènes. Remarquons que ce calcul de Hamilton ne tient pas compte du fait que les femelles non stériles donnent aussi naissance à des mâles (œufs non fécondés) qui partagent avec elles $50 \%$ de leurs gènes et qui sont susceptibles d'aller les répandre auprès d'autres colonies.
} 
à $X_{1}$, sauf s'il y a contrepartie, et que $X_{2}$ peut compter sur le soutien de $X_{1}$ s'il se trouve attaqué par un autre prédateur. Si cet altruisme réciproque (relativement peu coûteux) fonctionne, $\mathrm{X}_{1}$ et $\mathrm{X}_{2}$ ont tous les deux considérablement augmenté leur chance de survie et donc leur fitness potentielle. On comprend que les patrimoines génétiques prédisposant à de tels comportements (s'ils existent) aient été sélectionnés dans des environnements qui comprennent toujours des prédateurs. L'altruisme réciproque de Trivers n'est pas un acte de coopération simple dont l'avantage est immédiat, comme il en existe chez les prédateurs. Il relèverait plutôt de la logique du don ${ }^{3}$, les gènes prédisposant à ce comportement (ou l'autorisant) ayant été favorisés par la pression de prédation.

\section{La formalisation de John Maynard Smith}

Pour expliquer comment l'évolution a pu favoriser certains traits phénotypiques ou comportementaux, John Maynard Smith (1982 et 1989) a utilisé la théorie des jeux. Celle-ci peut proposer une modélisation formelle de la sélection naturelle, moyennant deux modifications par rapport à son application dans le champ de l'économie. Pour évaluer les conséquences, il faut d'abord substituer la fitness à l'utilité. Ensuite, en lieu et place de la rationalité instrumentale supposée des humains (celle qui les conduit à maximiser leur utilité) dans les applications économiques de la théorie des jeux, il faut postuler une stratégie stable d'évolution, c'est-à-dire que chaque individu s'en tient à une règle de conduite vis-à-vis d'un partenaire dont il ne connaît pas a priori les intentions mais dont il peut mémoriser les actes.

En répétant indéfiniment un dilemme du prisonnier entre des entités «égoïstes 》 (chacune cherchant à maximiser sa fitness), John Maynard Smith montre qu'il existe une règle de conduite optimale vis-à-vis de tout partenaire : c'est la règle du « donnantdonnant » ou du «tit for tat». Version proche de l'altruisme réciproque, cette stratégie du prêté pour un rendu consiste à coopérer au premier coup, et à se comporter ensuite comme le partenaire s'est comporté lors du jeu précédent. Une telle stratégie est stable, toujours gagnante en terme de fitness, et résiste à l'invasion de stratégies de défection.

Les tournois de dilemme du prisonnier de John Maynard Smith montrent ainsi qu'à partir d'hypothèses minimalistes (entités égoïstes, s'en tenant à une règle de conduite stable vis-à-vis de tout partenaire, mais susceptibles de réagir à l'attitude de celui-ci) on peut obtenir, par sélection sur la descendance, la diffusion de comportements complexes tels que le «tit for tat» et donc de formes de coopération et d'assistance ${ }^{4}$.

\section{Critiques}

En dépit de leur élégance, ces démarches ne sont pas à l'abri des critiques. Précisons que nous n'entendons pas ici développer une critique de la sociobiologie en tant que telle

\footnotetext{
${ }^{3}$ Nous entendons ce terme au sens que lui donne classiquement l'anthropologie. Il ne s'agit pas d'un geste de pure générosité, mais d'un acte qui a pour fonction (et pour objectif) d'inaugurer une chaîne de comportements : le don initie ainsi une série d'actes réciproques, d'obligations, de contre-dons.

${ }^{4} \mathrm{D}$ 'autres scénarios ont été, depuis lors, simulés avec de multiples individus dotés de nombreuses stratégies concurrentes, conduisant à sélectionner des comportements différents du donnant-donnant. Voir Novac et Simund (1993, pp. 56-58). Voir aussi, Kitcher (1993, pp. 497-516).
} 
mais, tout en admettant la légitimité de son ambition scientifique, nous voudrions mettre en évidence ses limites et insister sur certaines questions qu'elle laisse en suspens.

\section{Il y a là de belles machines à justifier l'existant}

On peut reprocher à la sociobiologie (mais c'est le cas de nombreuses recherches darwiniennes sur l'émergence de tel ou tel trait anatomique, physiologique, comportemental ou psychique) de fabriquer des machines à justifier l'existant. Soit un caractère constaté (et plus ou moins stabilisé) présent dans une espèce donnée. Ce caractère est présumé contrôlé par un mécanisme génétique. Il suffit de trouver un argument expliquant l'avantage qu'il peut accorder en matière de descendance pour expliquer sa présence par un mécanisme aveugle : celui de la sélection naturelle. Se proposant de répondre à la question du «pourquoi ? » (pourquoi tel caractère - et pas tel autre - existe-t-il dans telle espèce ?), la sociobiologie est à la recherche d'un finalisme, ou plus exactement d'une téléologie mécaniste.

Comment expliquer que l'individu soit amené à consacrer une partie de ses énergies à des activités collectives, sans qu'il y ait pour lui de bénéfice apparent, plutôt que de s'occuper d'assurer sa survie et sa descendance en exerçant l'ensemble de ses fonctions ? " $\underline{\text { S'il est vrai }}$ que la Nature ne fait rien en vain [souligné par nous], il faut trouver quel est l'intérêt qui a pu permettre la sélection de tels comportements » (Jaisson, 1993, p. 44).

Les sociobiologistes s'ingénient ainsi à inventer des hypothèses pour expliquer que les choses sont ce qu'elles doivent être (tout en admettant que, selon les contingences historiques de l'évolution, elles auraient pu être autrement). Si certaines de ces hypothèses se sont révélées fécondes, suscitant de nombreuses recherches pour les valider, ou les infirmer, elles tendent nécessairement à privilégier « la spéculation sur la fonction d'un phénomène aux dépens de l'interrogation sur les causes plus proximales : anatomiques ou neurophysiologiques, hormonales ou psychologiques » (Schäppi, 2002, p. 261). Or, comme le déplore Rolf Schäppi, les hypothèses phylogénétiques fonctionnelles sont plus difficiles à tester que celles que l'on peut avancer sur les causes proximales qui expliquent le « comment » des phénomènes. Enfin, il n'est pas rare que l'on élabore des arguments ad hoc pour expliquer l'avantage adaptatif qui justifie l'existence d'un trait quelconque ${ }^{5}$. C'est ce que reconnaît Daniel Dennett (2000, p. 603) à la fin de l'ouvrage qu'il consacre à la révolution darwinienne :

«Ma propre expérience m'a appris, et c'est embarrassant, combien il est facile de concocter des explications darwiniennes remarquablement convaincantes, qui fondent comme neige au soleil dès qu'on se livre à un examen plus serré ».

Mais, si l'on parvient ainsi à expliquer (plus ou moins bien) ce que pourrait être la raison d'être d'un caractère quelconque, cela ne signifie pas que les modèles sociobiologiques simulent les processus réels qui l'ont sélectionné au cours de l'évolution. Tous ces

\footnotetext{
${ }^{5}$ Dawkins n'est pas très éloigné de ce genre d'argument ad hoc, lorsqu'il tente d'expliquer la singularité de la ménopause chez la femme. Partant de l'idée que les chances de survie de l'enfant d'une mère âgée sont moindres que celles de l'enfant d'une mère jeune, Dawkins remarque qu'une femme partage la moitié de ses gènes avec ses enfants et le quart avec ses petits enfants. À partir de l'âge où les chances que son enfant parvienne à l'âge adulte et se reproduise sont inférieures à la moitié de celles qu'a son petit enfant d'y parvenir, la femme a intérêt, pour la postérité de ses gènes, à cesser d'investir dans sa propre reproduction pour se consacrer à son rôle de grand-mère. C'est ainsi que Dawkins explique que le pool génétique des humains a été envahi par un « grandchild altruism gene » (Schäppi, 2002, p. 301).
} 
modèles supposent implicitement la stabilité des conditions de la sélection qui s'exerce sur les gènes (et/ou sur les organismes). Or, sur le registre du temps long (celui de l'évolution), on ne saurait retenir une telle hypothèse. Par exemple, un caractère qui constituait un avantage dans le contexte géologique du Crétacé, ou dans celui des dernières glaciations du Quaternaire, peut devenir un handicap dans un contexte différent. La postérité des multiples caractères nouveaux qui ont émergé au cours de l'évolution a été contingente, relative au site dans lequel ils se sont inscrits. Selon les ressources disponibles, les concurrents, les parasites, les prédateurs et le régime des perturbations climatiques, un caractère peut constituer un avantage ou un inconvénient en terme de fitness : "l'avantage que confère une caractéristique particulière est donc toujours relatif - à un lieu, un temps - et peut être remis en cause de manière permanente » (Morange, 2005, p. 70). Faisant fi de ces contingences, les modèles sociobiologiques montrent sans doute que l'altruisme était possible sans que l'on soit obligé de mettre en cause la lutte pour la vie, mais ils n'expliquent pas comment ces caractères comportementaux ont historiquement émergé et ont été dans les conditions de se développer.

\section{Des pièges rhétoriques}

L'argumentation sociobiologique dépend d'une métaphore qui assimile les séquences de bases de l'ADN à un code et le génome à un programme, d'une autre qui qualifie tout gène comme une entité «égoïste », d'une troisième enfin qui use abusivement de la notion de «stratégie ». La métaphore du programme est sans doute un peu trop filée ; quant au terme d'égoïste pour qualifier le comportement des gènes, et à la notion de stratégie, ils sont ouvertement anthropomorphiques.

Le raisonnement sociobiologique (c'est particulièrement évident dans la théorie du " gène égoïste » de Dawkins) suppose qu'un gène correspond à un caractère. Or, les développements récents de la génomique ont appris qu'un gène contribue très souvent à l'expression de plusieurs caractères (physiologiques ou comportementaux) et qu'inversement, la plupart de ces caractères sont sous le contrôle d'un nombre a priori indéterminé de gènes en interaction les uns avec les autres. Si bien que, tant que l'on ne connaît pas le mécanisme génétique complexe qui pourrait prédisposer à l'expression de tel ou tel comportement dans un environnement donné, on ne peut pas savoir si la fitness constatée tient aux avantages accordés par ce comportement ou si elle est due aux caractères qui lui sont génétiquement associés. On ignore de même quels gènes seraient sélectionnés dans le cas où un comportement (mais cela est vrai aussi pour tout caractère physiologique ou anatomique) accorderait effectivement un avantage reproductif. Les développements de la génétique ne permettent plus de concevoir des gènes isolés et d'appréhender leur devenir indépendamment de celui de l'ensemble du fonds génétique dans lequel ils s'inscrivent.

Tous ceux qui réfléchissent sur l'animalité se font régulièrement opposer l'argument (quasiment injurieux) d'anthropomorphisme. L'accusation est si stigmatisante qu'elle conduit bien souvent les éthologistes à d'infinies précautions oratoires, dont se moque, non sans raison, Frans De Waal (1991 et 1997). On peut alors se demander pourquoi l'anthropomorphisme serait plus justifié quand il s'applique à une molécule de structure relativement simple comme l'ADN, que lorsqu'il s'agit de qualifier les états mentaux ou le comportement d'organismes disposant d'un système nerveux proche de celui des hommes. On peut, à ce sujet, relever que qualifier un gène d'égoïste suppose une 
déperdition de sens par rapport à ce que signifie l'égoïsme chez les humains. Lorsque l'on parle d'égoïsme à propos des humains, cela ne signifie pas que l'individu n'agit que pour maximiser son intérêt propre : il cherche certes à maximiser quelque chose que l'on peut qualifier de bien-être, et qui se décline en "préférences » hiérarchisées. Dans cette conception de l'égoïsme humain, rien n'empêche un individu d'estimer (il est seul juge de la chose) que son propre bonheur dépend de celui de ses proches, de la réussite d'individus qu'il admire, voire même de la promotion d'une cause « altruiste » à laquelle il adhère. Le calcul « égoïste » de l'intérêt peut donc, en principe, porter sur des " préférences » désintéressées. Bien évidemment, qualifier "d'égoïste » une molécule chimique, ne peut pas signifier que celle-ci agit en fonction d'une hiérarchie personnelle de ses « préférences » incluant éventuellement des « préférences altruistes ». Si l'on peut donc parler « d'égoïsme » au sujet d'un gène, c'est d'un « égoïsme » étroit qui vise à maximiser un intérêt propre, réduit à la propension à se dupliquer et à se répandre.

Des métaphores, comme celles du gène «égoïste » ou du terme de "stratégie » (issu de l'application de la théorie des jeux), ont sans doute une valeur heuristique, mais, comme l'argumente Michel Morange (2005, p. 78), elles présentent aussi des inconvénients.

« Les métaphores jouent un rôle important dans l'élaboration de la connaissance scientifique. Elles permettent la circulation des idées entre des domaines scientifiques différents, et même plus généralement entre la connaissance scientifique et la culture d'une époque. Mais elles ont le revers de leur médaille : elles sont, par nature, floues, imprécises. Leur trop grand usage peut obscurcir le champ des connaissances et rendre plus difficile la remise en cause permanente de celles-ci. Or, ce questionnement récurrent sur la valeur des connaissances est une part essentielle de l'activité scientifique (...) Aller au-delà des métaphores doit être pour tout scientifique un objectif permanent. »

Ainsi des métaphores comme celles du "programme génétique » et du «gène égoïste » ont entravé l'étude des interactions au sein du génome et de celles qui existent entre l'ADN du noyau et son environnement cytoplasmique. De même ont-elles occulté les mécanismes d'expression ou d'inhibition des gènes... Bref, l'ensemble des processus épigénétiques qui ont un rôle non négligeable dans les caractéristiques des organismes. Quant au raisonnement en termes de "stratégies », il aurait pour inconvénient, selon Michel Morange, de supposer que «l'espace ouvert à l'évolution des formes vivantes est presque infini, et que les contraintes qui orienteraient et limiteraient l'évolution sont négligeables » (Morange, 2005, p. 78). Or, il n'en est rien : non seulement il est des contraintes physiques que les organismes ne peuvent que respecter, compte tenu du milieu dans lequel ils vivent, mais en outre le patrimoine génétique de tout organisme (l'histoire évolutive qu'il porte en lui) limite considérablement les possibilités de variations.

\section{Les limites du déterminisme des comportements par le génome}

Dans la présentation que nous en avons faite, les modèles sociobiologiques qui proposent une justification fonctionnelle des attitudes «altruistes », ne tiennent pas compte des capacités requises par les comportements considérés. L'altruisme de parentèle de Hamilton suppose que l'organisme soit capable de reconnaître les individus qui lui sont apparentés, voire de distinguer ces individus selon la proportion de gènes qu'ils possèdent en commun. C'est pourquoi la théorie de Hamilton a impulsé de nombreuses et fructueuses recherches sur ces mécanismes de reconnaissance (Jaisson, 1993). 
L'altruisme réciproque de Trivers suppose que les individus considérés soient équipés de capacités cognitives qui leur permettent de reconnaître individuellement leurs partenaires, puis de se souvenir de leur attitude passée, enfin d'anticiper le renvoi d'ascenseur qu'ils en peuvent attendre. Il en est de même des capacités requises pour adopter la règle du «tit for tat». Or, ces capacités d'individuation, de mémorisation et d'anticipation, relativement rares dans le règne animal, peuvent servir à d'autres fins qu'à réguler les relations entre des individus appartenant à la même espèce. On ne peut donc savoir si la fitness de ces individus est due à leur comportement « altruiste » ou aux capacités cognitives requises par celui-ci... Capacités susceptibles de servir, sans que «l'altruisme» soit en cause, dans les interactions avec des concurrents, des proies ou des prédateurs.

Précisons ce point. Dès qu'apparaît dans une espèce animale un dispositif de contrôle de l'activité par une représentation du monde environnant, l'organisme a les moyens de corriger son action (sa trajectoire, son comportement) en fonction des informations qui lui parviennent de la situation. À la différence des organismes qui se contentent de trajectoires balistiques (une fois lancé le mouvement vers une cible quelconque, l'animal ne peut plus le modifier) ou de tropismes aveugles (cas des plantes), l'animal qui dispose de capacités cognitives de représentation peut résoudre les problèmes qui se posent à lui, et trouver de bonnes réponses (Proust, 1997). Pour peu qu'il dispose, en outre, de capacités d'apprentissage, il trouvera les bonnes réponses dans une grande diversité de situations, ce qui augmentera son adaptabilité, et finalement sa fitness. Mais son patrimoine génétique ne lui a pas dicté les bonnes réponses : il l'a simplement doté des aptitudes cognitives susceptibles de les chercher et de les trouver ${ }^{6}$. Dès qu'apparaît ainsi une capacité d'intentionnalité, on ne peut plus induire d'une réussite adaptative constatée, qu'elle résulte d'une sélection génétique directe. Cela signifie seulement que l'organisme considéré a hérité de l'équipement neuronal qui lui permet, soit de "réinventer la roue » à chaque occasion où cela s'avère nécessaire, soit même de transmettre " l'invention de la roue ", par un processus culturel, à ses descendants (Dennett, 2000, p. 559). Or, les bonnes solutions (les « roues » inventées ou transmises) peuvent concerner justement des règles de comportement vis-à-vis des congénères mais elles ne concernent pas qu'elles et rien ne permet de dire si l'avantage reproductif est principalement dû à l'attitude envers les individus de la même espèce ou à des capacités supérieures de se procurer de la nourriture et d'échapper aux prédateurs.

Pour une fois d'accord avec Steven Jay Gould, Dennett oppose ainsi à la sociobiologie qu'il y a « un obstacle fondamental (...) aux inférences qui ont cours en sociobiologie humaine : montrer qu'un type particulier de comportement humain est omniprésent ou presque omniprésent dans des cultures séparées, ne montre en aucune façon qu'il y a prédisposition génétique pour ce comportement particulier» (Dennett, 2000, p. 560). En effet, l'adaptation des humains n'a pas pour unique source la transmission des gènes,

\footnotetext{
${ }^{6}$ «Chez toutes les espèces complexes et évoluées chez lesquelles l'apprentissage joue un rôle important, le répertoire comportemental permet à l'individu de disposer de la flexibilité nécessaire pour répondre de façon adéquate à des circonstances différentes. Cette disposition ou potentialité est appelée adaptabilité [...] Certes au niveau d'organismes moins complexes, l'évolution est à l'origine de comportement génétiquement préprogrammés, souvent peu adaptables. Néanmoins l'évolution est également, surtout chez les espèces dites supérieures, source de flexibilité, de souplesse et de liberté. Les gènes sont responsables de la mise en place de structures physiologiques et nerveuses qui prennent la relève. Une fois cette délégation effectuée, ils ne déterminent plus directement le comportement » (Schäppi, 2002, pp. 207-208).
} 
mais aussi la transmission des objets, des techniques, des savoirs et des savoir-faire par la culture (une transmission lamarkienne, en quelque sorte, puisqu'il s'agit de caractères acquis). On ne saurait donc inférer de traits de comportement constatés ou de valeurs morales partagées, qu'ils résultent d'une sélection génétique. Dennett en conclut que toute théorie quant à la naissance des sentiments moraux et des règles de comportement morales devrait mettre en cohérence des processus biologiques et des processus culturels, et pas tout tenter d'expliquer par la seule biologie. Ce qui est évident pour l'homme, n'est pas moins vrai pour certains animaux.

« Même dans le cas des animaux non-humains, l'inférence effectuée à partir de l'adaptation pour conclure à une base génétique est risquée quand l'adaptation en question ne porte pas sur un trait anatomique mais sur un schéma comportemental qui est de toute évidence un Bon Truc. Car alors il y a une autre explication possible : la non-stupidité générale de l'espèce » (Dennett, 2000, p. 559).

Même si elles n'ont pas de transmission culturelle, certaines espèces animales peuvent fort bien « réinventer la roue » chaque fois que cela s'avère « un bon truc ». Bien qu'il soit partisan des travaux de John Maynard Smith, Dennett invite ainsi à prospecter d'autres voies (toutes aussi évolutionnistes) pour rendre compte de l'émergence et du développement de sentiments moraux et de règles de comportement que l'on pourrait qualifier de morales chez les animaux dotés des capacités cognitives requises.

Comme nous y invitait d'ailleurs Darwin, c'est donc chez les espèces animales phylogénétiquement proches d'homo sapiens et, comme le suggèrent les sociobiologistes, parmi les espèces sociales, que l'on peut rechercher les origines de la morale et des sentiments moraux. S'intéresser ainsi aux primates dans leurs comportements sociaux ne revient pas uniquement à prendre en compte la «non stupidité générale de l'espèce » et donc la possibilité qu'ont les individus de trouver de bonnes règles ou de « bons trucs », mais à se demander si ces bonnes solutions ne sont pas aussi partiellement transmises par des voies « culturelles ».

Remarquons enfin que les démarches sociobiologiques tendent à rendre compte de certaines régularités comportementales, mais pas des variations. On pourrait certes envisager des modèles plus complexes, cherchant à intégrer les interactions des patrimoines génétiques des organismes avec leurs environnements pour expliquer des différences comportementales entre populations isolées de la même espèce. Mais ce qu'apprend justement l'éthologie des primates, c'est qu'il y a des variations de comportement au sein de leurs populations ou entre des populations voisines, qui ne s'expliquent ni par des différences génétiques significatives, ni par un contexte écologique différent.

\section{La morale des chimpanzés}

Dans ses études sur les comportements sociaux des primates, Frans De Waal fait une approche cognitive de la gestion de la violence (agressivité et violence sont monnaie courante) et de l'apparition de la morale dans des sociétés de chimpanzés. Selon lui, ces primates sont réellement des êtres moraux : ils n'ont pas seulement des comportements que l'on peut qualifier de moraux par analogie avec ceux des humains; ils ont les dispositions (cognitives, émotives, expressives) qui autorisent à dire qu'ils agissent moralement. 


\section{Sentiments moraux et règles de comportement}

À la fin de Bon singe, Frans De Waal (1997, p. 266.) résume l'ensemble des caractéristiques qu'il a observées méticuleusement et qui l'autorisent à considérer les chimpanzés comme des êtres moraux.

\section{Phénomènes se rapportant à de la sympathie pour autrui}

Les chimpanzés ont une réelle aptitude à se représenter mentalement à la place des autres (ce que Frans De Waal appelle «l'empathie cognitive»). Elle se manifeste par des formes d'attachement à autrui, mais aussi par des comportements d'assistance et des phénomènes de contagion émotionnelle lorsqu'un membre de la population se trouve confronté à une situation critique. On relève de même une adaptation par apprentissage à la présence d'individus handicapés (de naissance ou par accident) : les chimpanzés adoptent à leur égard des comportements particuliers, de tolérance plus que de compassion, dont le résultat est de les protéger.

\section{Phénomènes apparentés à l'existence, à la transgression et au respect de normes de comportement}

Il y a, dans les sociétés de chimpanzés, des règles sociales prescriptives, dont certaines visent à stabiliser la structure hiérarchique de la population, quand d'autres tendent à faire respecter la réciprocité des actes. Ces animaux montrent, par leurs interactions, qu'ils ont la notion du don, mais aussi celle de l'échange (le donnant-donnant, l'attente d'un contre-don, et même la capacité à partager la nourriture sans attente précise d'une contrepartie). Ces règles sociales sont intériorisées et les individus savent anticiper la punition à laquelle ils s'exposent s'il les enfreignent. En effet, les agressions moralisatrices contre ceux qui violent les règles de réciprocité (ou ceux qui méprisent celles de la hiérarchie) ne sont pas rares.

Au-delà des conflits fréquents entraînés par la contestation des règles hiérarchiques d'accession à la reproduction, ces sociétés de chimpanzés manifestent un souci de la communauté et cherchent à préserver de bonnes relations entre leurs membres. C'est ainsi que l'on assiste à des interventions destinées à séparer les individus qui s'affrontent (même lorsqu'il s'agit d'une concurrence pour un statut de dominant), à des médiations qui ont pour effet d'éviter un conflit ou d'aboutir à une réconciliation.

On peut ajouter à cela, ce que Frans De Waal ne mentionne pas dans son tableau récapitulatif mais qui intervient à plusieurs reprises dans ses descriptions : les chimpanzés sont aussi capables de transgresser les règles, de tromper l'autre, de mentir, de feindre, de simuler et ce, dans des actions si compliquées que l'on doit leur supposer, comme il l'avait écrit quelques années plus tôt, une « intelligence stratégique » (De Waal, 1991, p. 173).

Le respect et la transgression des règles sociales, comme les modalités de régulation des conflits, supposent que ces animaux soient équipés d'une " conscience tripartite » (De Waal, 1997, p. 259) : dans son attitude envers B et C, l'individu A ne tient pas seulement compte des relations qu'il entretient habituellement avec eux, mais aussi des relations que $\mathrm{B}$ et $\mathrm{C}$ entretiennent entre eux.

\section{Critiques}

Ces travaux de Frans De Waal, dont les ouvrages se présentent comme des récits très agréables à lire, s'exposent à deux critiques principales. 
La première est l'anthropomorphisme. De Waal en fait état et se moque aimablement de ceux qui la formulent : quand il parle de « réconciliation scellée par un baiser », il reconnaît qu'en toute rigueur disciplinaire et pour éviter tout anthropomorphisme, il lui aurait fallu présenter la scène comme « une interaction postagoniste avec contact buccobuccal » (De Waal, 1997, p. 28). Plus sérieusement, il présente les protocoles d'observation et d'expérimentation qui lui permettent de parler "d'empathie cognitive ", "d'intelligence stratégique », de « réciprocité », de " médiations » et de " réconciliations ». Il appuie ses analyses sur un enregistrement (systématique et sur plusieurs années) des interactions observées et filmées dans son groupe de chimpanzés. Il procède alors à une décomposition analytique des séquences qui tranchent sur la routine des gestes quotidiens. Ce traitement des séquences et de leurs conséquences, complété par l'étude statistique de leurs occurrences, permet de justifier un premier niveau d'anthropomorphisme : l'analogie fonctionnelle de certaines attitudes, compétences cognitives ou interactions est telle que l'on peut s'autoriser à utiliser pour les qualifier chez les chimpanzé, les termes équivalents utilisés chez les humains. Ce n'est donc pas a priori et en guise d'hypothèse à valider, mais à l'issue d'une analyse systématique que Frans De Waal accepte, lorsqu'il décrit la vie sociale des chimpanzés, de parler de jeux, de parades, de saluts, de soumission, de réconciliation, de domination, mais aussi d'empathie et de sympathie. Si l'anthropomorphisme de Frans De Waal peut poser problème, c'est à un second niveau. Ayant acquis la connaissance des états mentaux des chimpanzés et de la façon dont ils les expriment, ayant en outre mis en évidence les règles de sociabilité qui sont propres au groupe, Frans De Waal met en récit l'histoire des phénomènes observés, prête à ses aspirants au statut de mâle dominant des stratégies complexes, impliquant alliances et ruptures d'alliances... Ce qui le conduit à se poser quasiment en Machiavel de ces sociétés de primates. C'est peut-être dans ces récits - qui font le charme de ses ouvrages - que l'anthropomorphisme de l'auteur serait le moins justifié.

La seconde critique tient à l'artificialisation du milieu dans lequel vivent les populations étudiées : sans doute évoluent-elles dans des zoos spacieux, mais il s'agit quand même de zoos. Cela jette un doute sur la possibilité de généraliser les résultats de ces observations systématiques aux populations naturelles, in situ. D'abord, il y a un problème (que Frans De Waal ne traite pas, sinon de façon allusive) d'interférences entre l'observateur et les animaux observés : non seulement ces interactions existent et peuvent modifier le comportement des chimpanzés, mais en outre, elles sont différentes de celles qui se produisent entre les primatologues de terrain et les primates en milieu naturel. Ensuite, il est une différence de taille entre le dispositif d'observation de Frans De Waal et le milieu naturel : ses chimpanzés sont nourris et protégés, alors que leurs congénères en liberté passent une grande partie de leur temps à rechercher leur nourriture et à se défendre d'éventuels prédateurs. D'où l'hypothèse que l'on pourrait émettre : ces chimpanzés s'ennuient, il n'est donc pas étonnant qu'ils passent leur temps à fomenter des révolutions de palais. Dans un tel contexte, il n'est guère surprenant, compte tenu de la "non stupidité générale de l'espèce », que ces primates aient trouvé les moyens de maintenir les conflits dans des limites susceptibles de préserver la cohérence de leur communauté.

Cependant, d'autres observations confirment la réponse de Frans De Waal à cette critique : il y a convergence entre ses observations et celles qui ont été réalisées en milieu naturel (même si les observations en milieu naturel sont moins systématiques et si les 
occurrences d'attitudes ne sont pas évaluées par d'imposantes séries statistiques comme dans le dispositif quasiment expérimental de Frans De Waal).

\section{Les leçons qu'en peut tirer la philosophie morale}

La première est que la morale des chimpanzés ne nous apprend pas plus de choses sur la moralité humaine que les modèles de la sociobiologie (les conclusions que tente d'en dégager Frans De Waal sont, de ce point de vue, plutôt insipides), mais que l'important est qu'il y est bien question des animaux, de leurs sentiments et de leurs comportements moraux. La seconde, plus indirecte mais plus surprenante, est que cette morale animale ne peut pas être qualifiée de morale naturelle.

\section{En quoi l'éthique est-elle concernée par ses origines « naturelles »?}

L'existence de sentiments et de comportements moraux chez les primates, comme l'émergence au cours de l'évolution et la sélection naturelle de conduites altruistes, ne nous éclaire guère sur les caractéristiques de la morale humaine. Les animaux ont manifestement la capacité de se repérer dans l'espace, certains d'entre eux celle d'effectuer des dénombrements sommaires (Hauser, 2002), mais l'arithmétique naïve des primates ne nous en apprend pas plus sur l'arithmétique, que les cartographies internes de nombreux animaux ne nous renseignent sur la géographie. C'est ainsi que le principal argument d'Henry Sidgwick (1999, pp. 164-233) contre tout naturalisme moral était que l'étude des origines de la morale n'a rien à voir avec les théories morales :

«J'ai évité d'entreprendre l'investigation de l'origine de la faculté morale (...) Si nous admettons que nous avons maintenant cette faculté, il m'apparaît que l'investigation des antécédents historiques de cette connaissance et de sa relation avec les autres éléments de l'esprit, n'appartient pas légitimement à l'Éthique, pas plus que les autres questions correspondantes sur la connaissance de l'espace n'appartiennent à la Géométrie »7 .

Qu'il y ait, du moins chez les primates, des sentiments et des comportements moraux signifie qu'ils ont hérité de capacités d'empathie et de sympathie. Ces capacités sont pré-requises pour former des intuitions et des évaluations morales, mais elles ne suffisent pas à développer une morale réflexive. C'est du moins l'argumentation que développe Vanessa Nurock (2006), dans la thèse récente qu'elle a consacrée aux origines et à la genèse du sens moral. Un individu ne peut avoir d'intuition morale que s'il est capable de se mettre à la place d'autrui. Il faut, par exemple, interpréter l'action de A sur B comme une action intentionnelle susceptible de nuire ou d'être bénéfique à $\mathrm{B}$ (ce que Vanessa Nurock définit comme «l'empathie agentive »). Il faut en outre percevoir les émotions, positives ou négatives, de $\mathrm{A}$ ou de $\mathrm{B}$ et réagir émotionnellement en s'identifiant à l'un ou à l'autre (ce qui est défini comme «l'empathie émotionnelle »). Selon la façon dont s'articulent les informations issues de ces deux capacités, l'individu est alors à même de former des évaluations morales "naïves ». L'hypothèse que Vanessa Nurock tente de vérifier est qu'il « existe au moins deux noyaux de morale naïve : l'un qui porte sur l'évaluation des actions susceptibles de causer un dommage physique à autrui, et l'autre qui concerne les actions liées à la réciprocité et à la coopération » (p. 442). Le premier

\footnotetext{
${ }^{7}$ Sidgwick, préface à la première édition de Methods of Ethics, p. V.
} 
noyau serait celui du « sens du bon et du mauvais » : l'action de A sur B interprétée par « l'empathie agentive » est évaluée grâce à " l'empathie émotionnelle », qui fait entrer le spectateur en sympathie avec les réactions de souffrance ou de joie de B. Le second noyau serait celui du «juste et de l'injuste » interprétée (par «l'empathie agentive ») comme acte intentionnel, l'action de A sur B est évaluée grâce à « l'empathie émotionnelle » qui produit chez le spectateur des réactions d'indignation ou de gratitude semblables à celles qu'éprouve B.

S'appuyant sur de nombreux résultats de psychologie expérimentale, Vanessa Nurock est en mesure d'affirmer que le «sens du bon et du mauvais » est précocement en place chez le jeune enfant ${ }^{8}$. Présent aussi chez le jeune enfant, le « sens du juste et de l'injuste » se développerait légèrement plus tard que celui du bon et du mauvais car il suppose la mise en place de capacités de mémorisation (réciprocité) et de décompte (répartition) ${ }^{9}$.

L'examen de la bibliographie tend à montrer que cette "morale naïve » est aussi présente dans le règne animal, du moins chez les primates. Ainsi les synthèses de Heyes (1998, pp. 101-134), celles de Preston et Frans De Waal (2002, pp. 1-72) ont établi que les primates sont dotés «d'empathie agentive» et «d'empathie émotionnelle». D'autres travaux (dont ceux, anciens, de Masserman ${ }^{10}$ ) témoignent de la présence chez les primates du « sens du bon et du mauvais » (De Waal, 1997) ainsi que du « sens du juste et de l'injuste » (Stevens et Hauser, 2004, pp. 60-65). On a donc de bonnes raisons de penser que les primates savent distinguer le bon du mauvais et le juste de l'injuste. Mais, s'ils sont ainsi dotés des deux «noyaux de morale naïve », cela ne signifie pas qu'ils soient capables (pas plus que ne le sont les bébés) de porter des jugements moraux. Pour ce faire il faut, en effet, disposer d'un système de second ordre : le sens moral, celui qui permet de réfléchir en termes de «bien et de mal» ou de « justice et d'injustice». Le sens moral traite bien sûr les évaluations issues de la "morale naïve », mais il doit aussi s'alimenter des informations provenant d'autres capacités (comme celles - déjà évoquées - de mémoriser les actes passés et de comparer des quantités). Deux de ces capacités joueraient un rôle important dans la formation des jugements moraux : « l'empathie situationnelle » et le langage. La première permet à l'individu qui en est doté d'appréhender les intentions et les croyances de plusieurs individus en interaction. Le second accorderait la faculté de lier entre elles et de mémoriser les évaluations issues de la « morale naïve » et aurait pour autre fonction de permettre à l'individu de comparer ses évaluations à celles de ses congénères. « Le langage joue très probablement un rôle dans la constitution des jugements moraux en rendant possible les échanges au sujet de ceuxci. Il permet aussi au sens moral d'opérer de manière réflexive dans les communications interpersonnelles portant sur les questions morales. Pour cette raison il agit de telle sorte que les intuitions fournies par la morale naïve se trouvent complétées et réévaluées en

\footnotetext{
${ }^{8}$ Dès 9 mois, le bébé est capable d'inférer le caractère intentionnel d'une action, de même reconnaît-il les expressions de détresse ou de joie chez autrui ; 6 mois plus tard il évalue différemment une action négative sur un objet d'une action négative sur un être vivant.

${ }^{9}$ À un an, le bébé saisit les conduites réciproques, à 18 mois les enfants sont capables de coopérer à une tâche. Mais il faut attendre quatre ans pour que l'enfant parvienne à partager de façon grossièrement équitable.

${ }^{10}$ Masserman et al., 1964, pp. 584-585. Masserman et ses collègues ont montré que des singes Rhésus préfèrent être affamés plutôt que d'obtenir leur nourriture en infligeant des décharges électriques à un congénère.
} 
fonction à la fois d'autres informations et des évaluations portées par d'autres individus » (Nurock, 2006, pp. 453-454).

Or « l'empathie situationnelle », qui ne se met en place qu'à partir de trois ans, serait spécifique aux hommes ${ }^{11}$. Le langage l'étant aussi, Vanessa Nurock en conclut que les primates ne peuvent réfléchir les évaluations de leur «morale naïve » et que le sens moral ne concerne que les humains. Même si les évaluations de la "morale naïve » sont authentiquement morales, elles ne sont ainsi que les « briques élémentaires » (celles dont les hommes ont hérité de leur histoire évolutive) sur lesquelles se construit la morale.

Certes, et cela permet de saisir la faible valeur heuristique d'une description de la morale des primates, si l'on entendait appréhender de la sorte l'éthique humaine. Mais, en s'interrogeant sur l'existence d'une capacité morale, individuelle et psychologique, Vanessa Nurock - comme Joëlle Proust (2003) dont elle reprend les conclusions à son compte - passe à côté de ce qu'apportent les recherches éthologiques de Frans De Waal : la morale ne se découvre, et ne s'exprime, qu'au sein de la socialité et l'on ne peut appréhender l'une sans l'autre. Or, si le langage la dynamise, la socialité ne le requiert pas nécessairement. On peut alors avancer l'hypothèse qu'il existe dans les sociétés de primates, des « communications interpersonnelles portant sur des questions de morale » qui ne sont pas langagières.

Si la question que la philosophie morale peut poser à l'éthologie est celle des prérequis de l'éthique, ce n'est donc pas à la même éthique qu'introduisent la sociobiologie (ou le cognitivisme) et la primatologie. La démarche sociobiologique revient à maintenir l'individualisme (méthodologique) et à fournir une genèse des conduites « altruistes » à partir de motivations étroitement intéressées. La démarche cognitiviste (celle de Vanessa Nurock, de Joëlle Proust ou de Marc Hauser) revient à rechercher dans les capacités cognitives et communicationnelles des individus, les conditions indispensables à l'émergence du sens moral. La primatologie, quant à elle, conduit à faire des sentiments moraux un prérequis des conduites morales, et donc à insister sur la dimension sociale de la morale. Dans la sociobiologie, comme dans les démarches cognitivistes, on trouve ainsi les présupposés des éthiques individualistes, et dans la primatologie ceux des éthiques communautariennes.

Il y a, de fait, une grande proximité entre la définition que donne Frans De Waal de la morale des primates (un certain consensus sur les comportements qui peuvent être tolérés ou qui doivent être interdits, celui qui se définit par la convenance, par l'attente sociale à l'égard des attitudes envers les congénères et par les formes de sanction des transgressions) et celle que donne Aldo Leopold (1933, pp. 634-43) : «An ethic, biologically, is a limitation on freedom of action in the struggle for existence. An ethic, philosophically, is a differentiation of social from anti-social forms of conducts » (repris dans Sand County Almanach (Leopold, 1949) en remplaçant «biologically » par « ecologically» et en introduisant le concept de « communauté biotique »).

Le rapprochement n'a rien d'étonnant. Dans les deux cas, la référence est darwinienne et l'on peut dire qu'avec son insistance sur les sentiments sociaux et sur le sens de la communauté, Frans De Waal est plus proche de Darwin que ne le sont les sociobiologistes

\footnotetext{
${ }^{11}$ Sur ce point Vanessa Nurock est en opposition avec Frans De Waal : ce que ce dernier définit comme « conscience tripartite» et qu'il a observé à l'œuvre chez les chimpanzés est une forme d'empathie situationnelle.
} 
et les cognitivistes. L'entretien qu'il a accordé au journal La Croix ${ }^{12}$ semble d'ailleurs répondre à l'ambition qui fut celle de l'auteur de la Filiation de l'homme : « Je veux démontrer que les sentiments moraux existent dans la nature, que tout n'est pas purement égoïste et qu'il y a continuité entre ces sentiments et notre morale. Je me place dans une perspective darwinienne : pourquoi la morale échapperait-elle à l'évolution ? Enfin, les instincts sociaux, qui furent sans nul doute acquis par l'homme comme par les animaux inférieurs pour le bien de la communauté, lui auront donné depuis le début quelque désir d'aider ses semblables, quelque sentiment de sympathie envers eux et l'auront porté à être attentif à leur approbation et à leur désapprobation. De telles impulsions lui auront servi, dans une période très ancienne de règle grossière du bien et du mal ».

\section{Une morale naturelle?}

Peut-on alors qualifier de «naturelle » cette éthique animale ? C'est ce que suppose Frans De Waal ${ }^{13}$ et c'est ce que l'on peut contester. En tout cas, l'éthique que pratiquent les chimpanzés qu'il étudie, remet en cause toutes les visions habituellement reçues de ce que serait un fondement naturel de l'éthique (ce que l'on pourrait appeler le naturalisme naïf, qui prétend isoler des racines naturelles de la morale, sans pour autant se livrer à de véritables observations éthologiques). On peut tenter de faire la liste de tous ces préjugés dits «naturalistes » qu'infirment les comportements moraux des chimpanzés.

- Ce n'est pas la loi (ou le droit) du plus fort : certes la supériorité physique peut jouer (dans la différence des rôles sociaux - ou de la « division du travail » - des sexes, particulièrement), mais elle n'est pas décisive. On le voit, par exemple, dans les conflits entre deux mâles pour la dominance : non seulement ce n'est pas nécessairement le plus fort qui domine, mais le conflit ne se règle jamais par un combat décisif (De Waal, 1991). Les chimpanzés maîtrisent fort bien les coups qu'ils se portent.

- On ne trouve pas non plus un ordre hiérarchique préexistant, dans lequel viendraient se loger les différents individus, ou les différents groupes et qui fixeraient de façon rigide leurs relations (ce que se figurent certains lecteurs de Platon, ou certains admirateurs des insectes sociaux $\left.{ }^{14}\right)$. Ce que montre Frans De Waal, c'est que, s'il y a une hiérarchie dans les sociétés de chimpanzés, celle-ci n'est pas donnée une fois pour toutes et ne se règle pas sur des caractéristiques préétablies ; elle est le résultat temporaire de réajustements constants, passant par des conflits et des règlements de conflits. Chez les chimpanzés, les relations de dominance peuvent être contestées dans certains cas, sans que cela conduise au chaos.

- Il n'y a pas de caractéristiques universelles, qui permettraient de dire que tel comportement moral est ainsi «par nature », mais au contraire des variations d'une espèce à l'autre (les macaques rhésus ne supportent pas des manquements à la

\footnotetext{
${ }^{12}$ F. De Waal, entretien avec Sophie Laurent, La Croix, 21 Juin 1997.

${ }^{13}$ On peut constater, de ce point de vue, deux attitudes symétriques (mais peut-être pas compatibles) chez Frans De Waal: d'une part, une tendance, maîtrisée, mais indubitable, à l'anthropomorphisme (appliquer des catégories humaines aux conduites animales) ; d'autre part, une tendance à qualifier de " naturels » les comportements communs à l'homme et à l'animal, comme si la catégorie exportée de l'homme à l'animal, une fois validée du côté de l'animal, revenait vers l'humain avec le qualificatif de «naturel». Ce terrain commun entre l'homme et l'animal, qui permet la réexportation de ce qui avait été importé, ne doit-il pas être considéré autant comme culturel que comme naturel ?

${ }^{14}$ Comme Edward O. Wilson, par exemple.
} 
hiérarchie que les chimpanzés acceptent, les bonobos sont encore plus tolérants vis-àvis de ces transgressions et plus pacifiques dans leurs relations) ou à l'intérieur d'une même espèce (une femelle peut, dans certaines circonstances, se trouver en position de dominance).

- S'il y a une « méchanceté » animale, celle-ci n'est pas assignable à une nature, ou à un instinct. C'est l'interprétation que fait Frans De Waal de la violence et de l'agression, ce en quoi il s'oppose à Konrad Lorenz. Les conflits accompagnés de violence existent, mais ils ne sont pas attribuables à un instinct, ni ne doivent être étudiés isolément dans un individu : ils ne se comprennent qu'à partir des relations sociales où ils apparaissent, et dans lesquelles ils n'ont pas un rôle uniquement négatif, ou destructif, car ces conflits permettent d'établir de nouvelles relations.

Dans ces conditions, peut-on parler encore d'une éthique « naturelle »? La thèse de Dominique Lestel (2001) est que, lorsque les variations de comportements à l'intérieur d'une même espèce sont nombreuses, qu'elles s'accompagnent d'une individuation forte (les chimpanzés, par exemple, ont des personnalités très marquées) et ne peuvent s'expliquer par des facteurs génétiques ou environnementaux, elles doivent être considérées comme culturelles : elles sont conservées dans des traditions (de population ou de lignage au sein d'une population) et transmises par imitation et éducation des petits. C'est pourquoi Dominique Lestel affirme que « l'avenir de l'éthologie se trouve autant du côté des sciences sociales que de la biologie» (2001, p. 13 ; voir aussi p. 290).

\section{Des origines culturelles de la morale dans le règne animal ?}

Pour établir sa thèse, Dominique Lestel se réfère principalement à des travaux qui montrent qu'il existe chez différentes espèces de primates des "cultures techniques », transmissibles par expérience au sein des populations et différentes d'un groupe social à un autre ${ }^{15}$. Il resterait à préciser ce que peut apporter cette approche culturelle des techniques à une étude des comportements moraux. Mais on peut considérer que l'hypothèse des origines animales de la culture et de la morale a un triple aspect.

D'une part, une dimension cognitive : les conditions (intentionnalité, représentation du monde, distinction sujet/objet, réciprocité et capacité à s'identifier à un autre sans le prendre comme un objet) qui permettent d'attribuer à certains animaux une subjectivité (et une intersubjectivité). On peut ainsi inscrire la démarche de Dominique Lestel dans le prolongement des sciences cognitives et, par exemple, des travaux de Joëlle Proust (1997). Celle-ci ne se préoccupe pas de savoir s'il y a des sentiments ou des comportements moraux chez les animaux. Son propos est de savoir si les animaux ont la capacité de former des représentations de leur environnement et des événements qui s'y produisent, pour agir et au besoin pour rectifier leur action. À l'issue d'une longue et prudente enquête (craignant la stigmatisation d'anthropomorphisme, elle s'est imposée une «mesure d'austérité » (Proust, 1997, p. 14) et a recherché si l'on peut trouver chez les animaux les «conditions minimales » pour que l'on puisse parler d'activité mentale, de représentation et d'intentionnalité), Joëlle Proust est en mesure d'établir que certains animaux ont un «point de vue subjectif sur le monde ». Cela signifie précisément qu'ils peuvent distinguer leur expérience mentale de l'objet de cette expérience, et qu'ils ont

\footnotetext{
${ }^{15}$ Il évoque ainsi, et parmi de nombreuses références, les travaux de Mc Grew, 1992, et ceux de Wrangham et al., 1994.
} 
des motivations diverses qui les poussent à agir dans le cadre des représentations qu'ils se font de la situation dans laquelle ils sont pris. Il s'ensuit qu'il existe chez certains animaux supérieurs une capacité à «maîtriser l'objectivité », et donc la possibilité de parler (sans être stigmatisé d'anthropomorphiste) d'une « subjectivité animale » (Proust, 1997, pp. 229-230). C'est bien sûr l'hypothèse d'une telle subjectivité que Dominique Lestel fonde celle d'une possible existence de «cultures » chez les animaux les plus évolués (primates, mais pas uniquement).

D'autre part, il faut pouvoir qualifier l'animal (dans certains cas) comme un sujet moral. Ce que refusent par contre Joëlle Proust et Vanessa Nurock, mais qui est fortement suggéré par Frans De Waal. Une démarche comme celle de Dominique Lestel ne conduit pas à faire de tous les animaux des sujets pleins, ou forts (les épinoches ou les drosophiles ne sont pas des êtres moraux), mais elle suppose une approche différente (attentive aux variations, ne recherchant pas l'explication fonctionnelle à tout prix) des conduites animales qui, non seulement étend la sphère des comportements culturels au-delà des cas les plus connus, mais conduit à un renouvellement du regard sur les comportements observés.

Il faut enfin considérer la dimension sociale de ces comportements moraux. Chez les chimpanzés; il n'y a pas de hiérarchie fixe, pas d'imposition de la force brute, mais un réseau mouvant d'interactions plus ou moins complexes et essentiellement, réciproques. L'unité en semble être ce que Frans De Waal désigne comme un «contrat ». Il ne s'agit pas d'un contrat originaire, mais d'un rapport, biunivoque et temporaire, d'avantages mutuels qui règle les échanges. Pour désigner ce «contrat» Frans De Waal utilise des métaphores comme le «marché » (Politique des chimpanzés) ou la " bourse » (Le bon singe). Serait-ce à dire que l'on retrouve en primatologie et sur cette question quelque chose qui rapproche du dilemme du prisonnier et de la façon dont les sociobiologistes transfèrent la théorie des jeux de l'économie à l'évolution?

On peut proposer une autre explication, celle qu'avance Michel Serres dans Éclaircissements :

«Chimpanzés, babouins [...], termites ou castors, les animaux concluent sans cesse entre eux des contrats purement sociaux, vides, exclusivement fondés sur le nous; la légèreté de ces conventions les oblige à les contracter en temps réel, continûment ; voilà, bien défini, l'accablement, strictement politique, où se trouvent plongées les sociétés animales. L'hominité commence avec la lourdeur de l'objet, d'où le nouveau contrat social reçoit une pesanteur dont la densité ouvre une histoire imprévue, autre que la répétition de la même convention » (1994, p. 287).

Pour comprendre aussi bien la répétition du contrat que la manière d'en sortir, il n'est pas besoin d'un algorithme, mais d'une histoire, une histoire qui ne soit pas seulement naturelle, mais sociale et culturelle, de la morale. La technique peut y jouer un rôle important, non point parce qu'elle est un rapport aux choses, mais en raison de sa capacité à fixer les rapports sociaux : la technique est un rapport social matérialisé. Converge avec la remarque de Michel Serres, ce que dit Dominique Lestel sur la différence entre la société complexe des babouins et la société compliquée des humains : faute de culture matérielle, et donc sans l'usage d'artefacts, les babouins ont à renégocier sans cesse leurs relations sociales (Lestel, 2001, p. 92 ; Lestel et al., 1999, pp. 367-407). Peut-être que les chimpanzés qui disposent d'outils sommaires et d'une culture matérielle ont-ils moins à renégocier leurs rapports que les babouins? 


\section{En guise de conclusion}

De cet examen critique de la façon dont est comprise l'émergence d'intuitions et de conduites morales chez les animaux, nous tirerions volontiers deux conclusions qui ouvrent autant d'incertitudes qu'elles n'apportent de réponses à toutes les interrogations suscitées par l'hypothèse darwinienne de l'origine naturelle de la moralité.

Premièrement, la morale des animaux, y compris celle de nos plus proches cousins (les primates et, plus proches encore, les chimpanzés), n'est d'aucun secours, comme l'avait bien vu Sidgwick, pour appréhender notre morale, moins encore pour justifier des jugements moraux dans les affaires humaines. Mais cela concerne, par contre, la dimension éthique des rapports que nous entretenons avec les animaux. Que certaines espèces animales (dont les primates) disposent d'une «morale naïve», et donc des «briques élémentaires » sur lesquelles nous avons réfléchi et élaboré nos propres jugements moraux, ou que l'on puisse découvrir dans leurs sociétés un authentique " sens moral », nous ne pouvons nous comporter envers elles comme nous le faisons à l'égard d'animaux dont nous devons simplement respecter la sensibilité. Mais, s'il convient de respecter la morale (serait-elle « naïve ») de l'autre, encore faut-il la connaître. Et, pour cela, il reste encore beaucoup d'investigations à faire et de controverses en vue.

Deuxièmement, faire connaître l'éthique des animaux serait moins « naturaliser la morale », que chercher si l'on ne découvre pas des «cultures » chez les primates (et éventuellement dans d'autres espèces aux capacités cognitives développées). Ce serait donc « culturaliser l'animal». Et, pour cela aussi, il reste beaucoup à faire. 



\section{Chapitre 10 \\ Éthique et éthologie : comportement animal et conduite humaine}

JeAN-PhILIPPE PieRron

Dans l'abécédaire de la philosophie, la lettre A comme ailleurs est une invitation au voyage. Aller voir ailleurs si l'on s'y trouve prend l'allure du déplacement dans l'espace avec l'ethnologie ; de l'exploration des limites ultimes de l'espèce, l'animal en constituant la fine pointe, avec l'éthologie. Ainsi, à l'ailleurs dans l'espace fait écho l'ailleurs des espèces. Mais l'animal connu hier en ses éléments (modélisation mécaniste de la biologie) fait place à l'animal compris en son comportement (modélisation systémique de l'éthologie), reposant alors le sens du détour par cet autre de l'homme pour penser la part de l'autre en l'homme. Les comportements animaux désignent-ils alors les limites extrêmes des conduites humaines ? Y a-t-il un point d'intersection où le presque-humain qu'est l'animal partage ce déjà-plus un animal qu'est l'homme ? Entre le comportement animal et la conduite humaine, l'ethos habite-t-il cette intersection ? Rechercher une continuité entre l'action volontaire et son substrat involontaire et préréfléchi est-ce nécessairement céder au naturalisme?

«La vertu morale (éthique) est le produit de l'habitude, d'où lui est venu aussi son nom, par une légère modification de $e^{2} \cos ^{1}$ ». L'étymologie douteuse du mot " éthique » donne à penser que, s'il n'y a pas d'éthique sans éthologie, l'éthique ne se réduit pas à une éthologie. L'ethos, les mœurs sont porteurs d'homonymies accidentelles ${ }^{2}$. De fait, quelle signification accorder aux «mœurs» lorsque l'éthologue parlera de «vie et mœurs des abeilles », l'ethnologue des mœurs amérindiennes, ou le philosophe de fondements de la métaphysique des mœurs ? ${ }^{3}$ Envisageant le statut de l'ethos, dans une éthique

\footnotetext{
${ }^{1}$ Aristote, 2001, Éthique à Nicomaque, 1103 a 17, p. 87.

${ }^{2}$ Aristote, 2001, Éthique à Nicomaque, 1096 b 25, p. 51.

${ }^{3}$ La distinction hégélienne entre Moralität et Sittlichkeit, la morale et les mœurs précisera ultérieurement cette dialectique entre la visée de la vie bonne et les contextes moraux objectifs que sont les mœurs.
} 
introduisant une politique du monde humain comme monde commun (la polis), Aristote interroge le rapport entre communauté animale et société humaine.

« Il est évident que l'homme est un animal politique, bien plus que n'importe quelle abeille ou n'importe quel animal grégaire. Car, nous le disons souvent, la nature ne fait rien en vain. Et seul parmi les animaux l'homme est doué de parole.[...] C'est ce qui fait qu'il n'y a qu'une chose qui soit propre aux hommes et les sépare des autres animaux : la perception du bien et du mal, du juste et de l'injuste et autres notions de ce genre ; et avoir de telles notions en commun, voilà ce qui fait une famille et une cité4. »

La recherche du genre voisin et de la différence spécifique, inaugure la controverse sur les frontières humanité-animalité entre l'option continuiste recherchant le genre commun et l'option discontinuiste isolant la différence spécifique (langage, technique, éthique et politique). Ainsi, la distinction entre la voix de la parole, préfigurant la distinction contemporaine entre énoncés injonctifs, déclaratifs (échanges d'informations), et dénotatifs (échanger sur les signes eux-mêmes), rappelle qu'en philosophie morale l'homme qui a la parole est aussi un homme de paroles (pouvoir promettre signale l'importance de la parole dans la vie morale.) Il s'ensuit qu'une communauté animale, dans la dimension organique des liens qui la structurent, devient une société voire une cité, lorsque les liens et les mœurs qui l'organisent sont discutés, représentés et maintenus vivants dans une mémoire rediscutée. Sans doute est-ce là ce qui distingue la mémoire cumulative et sédimentée des «cultures animales » (Lestel, 2001 ; de Fontenay, 2000) de la mémoire dialectisée des cultures humaines faites de fidélité et d'oubli.

Mais quel est le statut des mœurs ? Les mœurs sont-elles la part la plus animale des conduites humaines, ou la part la plus humaine des comportements animaux ? Entre ici en débat la part de la nature et de l'histoire dans les comportements animaux et les conduites humaines. Certes, en disant « que l'homme est un animal politique bien plus que n'importe quelle abeille ou animal grégaire », Aristote dissolvait le concept d'animalité présenté comme une réalité homogène opposée frontalement à l'humanité, pour pointer qu'il n'y a pas l'animalité mais des animaux (insectes sociaux, troupeaux, colonies et hordes, etc.), dans un éclatement des genres. L'animal se dit en plusieurs façons. Ce n'est que sous l'effet d'une réification métaphysique que l'on parle de l'animalité comme d'une totalité servant de vis-à-vis à l'humanité. Mais dans sa réflexion éthique, Aristote est surtout attentif aux formes stables présentes dans les conduites humaines (l'hexis ${ }^{5}$ ), désignant la part de l'ethos sous l'éthique, des mœurs dans la morale. Est-on autorisé alors à parler d'un soubassement éthologique de l'éthique, observable chez les animaux, perdurant chez l'homme? La difficulté est de savoir si l'hexis, et avec elle les mœurs, constituent une fond de passivité dans la conduite humaine ou si elles jouent un rôle actif? Or, pour Aristote, les dispositifs d'action sont aussi des dispositions à agir. La dimension objective des conduites dans les mœurs - les usages comme style de conduites stabilisées, voire fossilisées -, n'annule pas leur dimension subjective dans la mesure où elles ouvrent les conduites sur des initiatives. C'est cette présence active des mœurs, sous l'effet d'une analogie fonctionnelle ${ }^{6}$, qui rapproche comportement animal et conduite

\footnotetext{
${ }^{4}$ Aristote, 1983, La Politique, pp. 40-41. C'est nous qui soulignons.

${ }^{5}$ Aristote, 2001, Éthique à Nicomaque, IV, 4, 1122 b 1; II, 4, 1106 a 12.

${ }^{6}$ Commentant Aristote, Simondon, 2004, p. 49, écrit : « L'habitude est chez l'animal une sorte d'expérience qui imite la prudence humaine. Imite cela veut dire, qui est l'analogue fonctionnel de la prudence humaine, mais avec des modes opératoires différents ».
} 
humaine (agir prudentiel). Mais la signification fonctionnelle de l'analogie n'autorise aucunement à en conclure à une signification ontologique. Les mœurs ne sont pas en nous comme des choses que l'on possède. Si nous en sommes porteurs, elles sont aussi portantes, opérant comme des interprétants par lesquels se mieux comprendre et expliciter la signification et la valeur de ses conduites ${ }^{7}$. La composante passive, la répétition et les éléments stéréotypés, présents dans les mœurs humaines et animales, ont une dimension active : la répétition permet l'approfondissement de sa conduite et prépare à l'innovation pratique. Ressassement, la répétition est aussi approfondissement. En ce sens, les mœurs entendues comme dispositions acquises, sont un point d'appui pour la vie morale.

\section{La révolution éthologique}

L'éthologie n'est plus sous la tutelle de la philosophie morale (elle trouvait place avant 1950 aux côtés de la caractérologie), mais sous celle des sciences du vivant ${ }^{8}$. L'éthologie scientifique comparée étudie la part des mœurs dans l'économie générale de la vie morale, assumant sur le terrain scientifique des questions jugées hier métaphysiques et éthiques. L'étude objective des mœurs a pour enjeu rien moins que la liberté humaine. Si une action n'est pas une réaction, ni une initiative une répétition, une conduite ne sera pas qu'un comportement. Or, en ces temps peu métaphysiciens, l'usage social des sciences leur délègue la fonction de grands récits. C'est pourquoi, si l'éthologie pose des questions épistémologiques, elle assume en fait des problèmes métaphysiques. La science normale joue le rôle de grande mythologie, quant à notre origine (la paléontologie pose la question de l'origine de l'homme en termes de commencement phylogénétique), notre statut ontologique (le matérialisme de la biologie moléculaire rappelle que le patrimoine génétique humain est pour l'essentiel patrimoine mondial de tous les vivants) et notre liberté (l'éthologie trouve dans nos conduites le rôle déterminant de schèmes comportementaux archaïques). L'éthologie, comme science du comportement et des mœurs animales, voire l'étude comparée des mœurs, active une orientation métaphysique qui a avantage à s'expliciter, les ontologies non critiquées portant d'ordinaire des dogmatismes inavoués. De fait, l'éthologie inflige une quatrième blessure métaphysique à l'humanité. Après la révolution héliocentrique galiléo-copernicienne perdant l'homme dans un univers infini, la fin de l'anthropocentrisme par un darwinisme inscrivant l'homme dans une évolution du vivant qui l'embrasse et le dépasse, et le freudisme affirmant que l'homme n'est pas l'être conscient et transparent qu'il prétend être, soumis qu'il serait à des forces inconscientes, l'éthologie ruine l'idée d'une autonomie morale assise en raison pour découvrir ses assises animales. La nature de la règle serait d'être de la nature. L'éthologie est, en ce sens, une herméneutique du soupçon eu égard aux conduites humaines, cherchant à en faire apparaître les soubassements neurophysiologiques, traquant le comportement animal sous la conduite humaine. De plus, la place faite à l'éthologie n'est pas sans liens avec le désenchantement du monde d'une société sécularisée. Si l'animal fascine l'éthologue, c'est que, dans une inversion notable, le bonobo aujourd'hui joue le rôle du pongo au XVIII ${ }^{\mathrm{e}}$ siècle. À l'homme mesure de toutes choses d'hier - le pongo est-il un homme

\footnotetext{
${ }^{7}$ Voir sur ce point 1'article de Pierre Rodrigo, 2004, pp. 11-26.

${ }^{8}$ K. Lorenz, K. von Frisch et N. Tinbergen seront consacrés, en 1973, par le prix Nobel de médecine et de physiologie« pour leurs travaux sur les causes et l'organisation des schèmes comportementaux. »
} 
des bois ? - a fait place l'animal mesure de toutes choses - l'homme serait-il un bonobo qui s'ignore ? - On disait hier à l'animal : «parle et je te baptise ». Aujourd'hui la certification éthologique tient lieu d'authentification théologique. Le discours éthologique contemporain entérine une sécularisation profonde aux incidences éthiques et métaphysiques considérables, l'homme devenant parfois un parasite, au mieux un symbiote, une anomalie taxinomique en tout cas $^{9}$. Consacre-t-il la mort de l'homme ou la mort d'une idée exaltée de l'homme ? Si l'homo sapiens sapiens, l'homme de la sagesse des sagesses, consacrait l'irruption du métaphysique dans le monde physique, y renoncer signifiera-t-il qu'il faille inscrire l'humain comme vivant parmi les autres vivants ?

L'éthologie porte une révolution épistémologique concernant le comportement animal. Elle promeut un nouveau paradigme théorique, se séparant du modèle cartésien de l'animal-machine qui substitua à l'ontologique Vie, une logique du vivant. Autorisant une « profanation » de la nature rendant théoriquement possible une maîtrise du vivant jusqu'à son expérimentation et son industrialisation, sans être un pur réductionnisme, le modèle de l'animal machine permit de mesurer combien l'animal, être passionnant qui résiste en son mystère, est aussi un objet passionnel qui encourage toutes les projections. Sur ce point, l'actuel tamagotchi, animal virtuel capable de naître, d'être prénommé, nourri, soigné, cajolé, de se reproduire et de mourir virtuellement, est l'animal machine fait réalité, bien plus que l'automate de Vaucanson. L'usage de ce pur automate qu'est le tamagotchi révèle la puissance omniprésente des projections débridées et sans complexes de l'anthropomorphisme. Lorsque l'homme parle de l'animal, il parle essentiellement, de lui-même. Il faut s'en souvenir lorsque l'on fait le détour de l'éthologie pour penser l'éthique. Modéliser le vivant comme une machine revenait à chercher à expliciter les conditions de possibilité rendant légitime un discours sur l'animal. Prudence cartésienne qui élabore et procède par induction négative : si on ne sait pas ce qu'est l'animal, on sait ce qu'il n'est pas. Son agnosticisme méthodologique interdit donc d'en affirmer plus qu'on en sait, laissant les hypothèses n'être que des hypothèses, et les « il semble » n'être que des semblances.

Quant à elle, forte de cette leçon, l'éthologie révise notre conception de l'animal. Une machine connaît tout au plus du mouvement - auto-mate -, mais pas de comportement, encore moins une conduite. À la fiction théorique de l'animal-machine, pure substance étendue (figure et mouvement, l'animal ne serait que de l'espace dans l'espace, répondant à un stimulus spatial par une réaction spatiale), l'éthologie oppose un nouveau paradigme : l'animal compris à partir de ses interactions avec son milieu, dans la constitution de son « éthogramme ${ }^{10}$ ». Lorenz et Uexküll ont découvert de façon décisive que l'espace

\footnotetext{
${ }^{9}$ Cf. Agamben, 2002, p. 44, commentant le travail de Linné : « Il convient de réfléchir sur cette anomalie taxinomique (l'idée d'homo sapiens) qui inscrit comme différence spécifique non une donnée mais un impératif... Définir l'homme non pas au moyen d'une nota characterestica, mais de la conscience de soi, signifie qu'est homme celui qui se reconnaîtra comme tel, que l'homme est l'animal qui doit se reconnaître humain pour l'être ».

${ }^{10}$ Le terme « éthogramme » a été introduit en 1936 par l'ornithologue Gerritt François Makkink dans un compterendu d'observation de l'avocette. L'éthogramme est un catalogue détaillé décrivant tous les comportements d'une espèce. Préalable à toute étude éthologique, il sert aussi à former le regard chez l'apprenti éthologiste, avec le problème de décider du meilleur découpage, du juste degré de détail à appliquer à la continuité des productions comportementales (Renck et Servais, 2002, pp. 65-66). Peut-on de 1'homme faire un éthogramme ? La question mérited'être posée parce qu'elle obligeà pointer la différence entre un comportementet une conduite. L'éthogramme de l'homme, qui devrait présenter de façon systématique et sans oubli l'ensemble des conduites humaines, serait du côté formel le code civil présentant dans l'abstraction de la loi l'ensemble des conduites dont les hommes sont capables, et du côté de l'intériorité un traité éthique des vertus...par définition inachevable. D'ailleurs, le concept d'empreinte écologique n'est-il pas la traduction économico-écologique de celui d'éthogramme ?
} 
de l'animal est un espace qualifié, qu'il existe des «mondes animaux », " un monde humain ». L'espace animal devenu un monde perçu n'est plus un contenant neutre où se déploieraient mécaniquement des comportements, mais un espace valorisé, investit par chaque espèce animale dans un plan de vie (Uexküll, 2004.). Plus pertinent que le concept téléologique de but, le plan de vie révèle des stratégies comportementales (techniques de chasse, répartition des reproducteurs, etc.) spécifiques. Le comportement animal sort donc du stéréotype, la nécessité de satisfaire des besoins n'excluant pas la flexibilité des solutions y répondant. L'éthologie montre qu'une forme d'innovation comportementale est possible. Désactivé de ses conséquences et implications éthiques (l'animal considéré comme matière première) et métaphysiques (l'animal comme chose), le modèle mécaniste de l'animal devient alors un outil méthodologique, utile dans les sciences du vivant, mais qu'on ne saurait réifier. Le modèle n'est pas la chose dont il est le modèle.

Entre une part animale de l'éthique et une éthique pour l'animale, l'enjeu est alors de savoir quelle est la part de l'éthologie dans la constitution de la vie éthique. Y a-t-il des éléments déjà présents dans les comportements animaux demeurant actifs dans les conduites humaines ? Si tel est le cas, cela a-t-il des conséquences éthiques quant à nos conduites à l'égard des animaux ? On se souvient alors de Montaigne, observant : « il se trouve plus de différence de tel homme à tel homme, que de tel animal à tel homme ». Ces questions mettent en jeu la consistance d'une différence anthropologique, à propos de la nature des mœurs. Assumant à nouveaux frais la question de la place de l'homme dans la nature et la part de la nature dans l'homme, "la question animale de l'homme » en éthique, n'oblige pas à un réductionnisme éthologique ramenant les conduites humaines à des comportements animaux. Parler de l'animal ne peut être pour l'homme autre chose que de prendre un point de vue humain sur l'animal, parce qu'il n'est pas possible de prendre le point de vue de l'animal. Un plan de vie n'est pas un point de vue. L'animal n'a pas la capacité de faire émerger son milieu comme un point de vue, comme un monde valant à part entière en dehors de ses comportements. C'est pourquoi l'homme nu n'est pas un singe nu. Merleau-Ponty écrivant sur la structure du comportement animal, refusait la réduction béhavioriste ne voyant dans l'animal qu'un montage de réflexes. Pensant le comportement animal en termes de forme complexe (interaction situation vitale/réaction instinctive), il ne cédait pas pour autant à la confusion des ordres. Parler d'un ordre du comportement animal et d'un ordre de la conduite humaine, c'est attester l'existence d'une différence anthropologique plutôt que d'une supériorité. Refuser d'en accorder trop peu à l'animal sans être tenté de trop lui en accorder parce qu'une différence d'ordre n'est pas nécessairement de l'anthropocentrisme.

«Si toutes les actions permettent une adaptation à la vie, le mot de vie n'a pas le même sens dans l'animalité et dans l'humanité, et les conditions de la vie sont définies par l'essence propre de l'espèce. Sans doute le vêtement, la maison servent à nous protéger du froid, le langage aide au travail collectif et à l'analyse du "solide inorganisé". Mais l'acte de se vêtir devient l'acte de la parure ou encore celui de la pudeur et révèle ainsi une nouvelle attitude envers soi-même et envers autrui. Seuls les hommes voient qu'ils sont nus. Dans la maison qu'il se construit, l'homme projette et réalise ses valeurs préférées. L'acte de parole exprime enfin qu'il cesse d'adhérer immédiatement au milieu, l'élève à la condition de spectacle et en prend possession mentalement par la connaissance proprement dite (Merleau-Ponty, 1977, p. 188). »

Le monde humain n'est plus un milieu de vie qualifié biologiquement, mais un univers symbolique. Le milieu devient un monde de valeurs transcrites et transmises 
dans et avec lequel comprendre ses émotions, interpréter leur signification et les juger en raison. Telles sont les mœurs humaines, qui, en tant que telles, ont une tout autre signification que les mœurs animales ${ }^{11}$. La dimension éthologique en nous, si elle part de la zoologie, devient événement biographique ayant valeur axiologique.

\section{L'éthologie et l'éthique philosophique}

Si l'homme est automate autant qu'esprit, incontestablement, l'éthologie rend plus lucide et moins ignorant quant aux causes qui déterminent l'agir humain. Ces automatismes pratiques qu'elle scrute invitent à penser un continuum entre la haute et sublime décision morale et les dispositions ordinaires, au contenu moral discret. En ce sens, il n’y a pas d'éthique sans une éthologie, l'école de la liberté étant une école de la lucidité. Une étude objective de nos mœurs a donc le mérite de décrire des comportements, moins pour nous abstenir d'avoir à nous déterminer que pour prendre la mesure du lieu propre où s'exerce notre agir. Mais, pour autant, l'éthique n'est pas réductible à une éthologie, car une régularité ne fait pas encore une légalité. Si l'éthologie décrit ce qu'il en est de nos mœurs et de ce qui les structure, l'éthique élucide et évalue ce qui devrait en être. L'éthologie explicite davantage ce qui travaille et structure nos conduites, mais savoir ce que l'on fait ne signifie pas encore savoir ce que l'on doit faire. Expliquer plus pour agir mieux, telle pourrait donc être une manière d'articuler les relations entre éthique et éthologie.

Penser cette articulation tient au fait que les sciences contemporaines redessinent les frontières homme/animal. La biologie moléculaire reconnaît un patrimoine génétique commun mais aussi l'existence de barrières interspécifiques (pas de reproduction). La paléontologie comparée découvre une phylogénétique mais sur fond de rupture et d'émergence spécifique foisonnante. L'éthologie complexifie notre compréhension des comportements animaux, mais n'en fait pas pour autant des conduites. La psychologie cognitive rapproche psychologie de l'enfant et psychologie de l'animal autour des apprentissages sans ignorer l'écart colossal entre psychologie de l'enfant et psychologie de l'adulte. Enfin, l'émergence du concept de «neuroéthique (Illes, 2006) interroge ce qu'il en est des stuctures neuronales comprises comme conditions nécessaires mais non suffisantes d'un discernement éthique. Mais l'urgence de penser les rapports entre éthique et éthologie est également motivée, outre par le mal que l'homme fait à l'animal (l'animal objectivé, réduit à sa matérialité avec l'animal de laboratoire ou l'élevage industriel), par le mal que l'homme fait à l'homme. La blessure terrible du XX $\mathrm{XX}^{\mathrm{e}}$ siècle est celle de la bestialité. Le mal que l'homme fait à l'homme est tel que Michel Terestchenko parle, à propos de l'homme, d' "Un si fragile vernis d'humanité » (2005). Cherchant la véritable motivation de l'agir du côté de l'égoïsme psychologique pour rendre compte de la banalité du mal, on est aujourd'hui tenté d'aller chercher du côté de l'animal le salut moral que l'on n'a pas trouvé du côté de l'humanité. À une humanité déconfite et défigurée, on opposerait alors une animalité vierge de tout soupçon, parce que toujours victime.

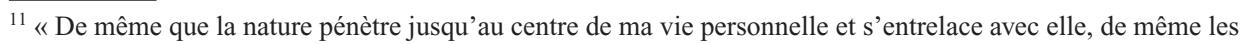
comportements descendent dans la nature et s'y déposent sous la forme d'un monde culturel. [...] La civilisation à laquelle je participe existe pour moi avec évidence dans les ustensiles qu'elle se donne » (Merleau-Ponty, 1967, pp. 399-400).
} 
Comment penser la banalité du mal, c'est-à-dire le fait que les ressorts éthiques n'aient pu empêcher l'extermination de l'autre homme ? L'animalité serait-elle un remède à la bestialité ? Si la morale fondée en raison s'est avérée impuissante, les émotions et les sentiments œuvrant dans les comportements animaux, ne sont-ils pas des ressources possibles ? Ainsi, après avoir exalté la raison comme fondement de la morale, faudrait-il maintenant la rabaisser, trouvant dans l'animalité de l'émotion ou du sentiment un fondement stable et innocent? Et l'on s'empressera de louer la générosité, l'empathie ou le sens de la «justice » dont les animaux seraient capables (De Waal, 2002 et 2006). Bref la tentation existe, après l'exaltation d'un humanisme triomphant qui vantait la différence spécifique, arguant de celle-ci pour justifier une domination violente de l'animal, de lui substituer un humanisme humilié. L'enjeu des relations entre éthique et éthologie est de trouver l'humilité d'un humanisme.

L'homme, seul animal capable d'être bestial, interroge les ressources susceptibles de réorienter une théorie de l'action. Une attention portée à l'animalité, en nous et en dehors de nous ( $c f$. le thème de la "pitié » chez Rousseau), c'est-à-dire sur la part des schèmes comportementaux, des émotions et des sentiments dans la vie morale, n'est-elle pas capable d'enrichir notre questionnement éthique et d'en réorienter la formulation, trouvant du côté du sentiment une mobilisation morale que la seule raison n'a pu apporter seule?

$\mathrm{Si}$, en aval, penser les relations entre éthique et éthologie débouche sur une éthique du souci de cet autre que nous qu'est l'animal, en amont c'est là l'occasion de redonner à une philosophie de l'action la part qui revient à l'importance des mœurs et à leurs soubassements neuronaux et moteurs, ainsi qu'au statut des émotions comme mobiles d'action. À la distinction classique en philosophie morale entre mobile et motif, - le motif étant rationnel et donc moral, le mobile étant un penchant d'ordre sensible -, l'articulation entre éthique et éthologie apporte une nuance. Kant n'avait retenu, en raison de l'économie même de sa propre conceptualisation de la moralité, pour seul mobile moral acceptable, un sentiment intellectuel, à savoir le respect. Si le pur respect pour la loi morale est un mobile en vue du bien, c'est aussi une brèche en ce qu'il questionne précisément la portée mobilisatrice d'autres mobiles (mœurs, émotions et sentiments) capables de nourrir notre envie d'agir en plus des bonnes raisons d'agir. S'il y a une évidence native de la bonne volonté, au sens kantien du terme, sur quoi cette évidence du bien repose-t-elle ? Kant la présente comme un fait de la raison mais rien n'interdit vraiment d'observer qu'elle est aussi, en partie, un fait de l'émotion.

«Les éléments constitutifs de la morale précédent clairement l'humanité. Nous les reconnaissons chez nos parents primates, l'empathie étant plus visible chez le bonobo, et la réciprocité chez le chimpanzé. Les règles morales nous disent quand et comment appliquer ces tendances, mais elles sont elles-mêmes à l'œuvre depuis des temps immémoriaux (De Waal, 2006, p. 270). »

Sans céder à la tentation d'une naturalisation de la morale qui guette un tel propos, reposant une manière $d^{\prime}$ ' " ontologiser » l'analogue, on peut toutefois se rendre attentif au rôle originaire des sentiments dans la vie morale. Sans parler d'un fondement naturel de la morale, voire de fondements d'une physique des mœurs comme il y a des fondements de la métaphysique des mœurs, on interrogera alors quelle est la part de la psychophysiologie dans la détermination des choix moraux et de la vie morale. Reconnaître l'idée d'un accompagnement moteur et émotionnel de nos options éthiques, invite à comprendre 
comment l'orientation brute que ce dernier est censé nous faire sentir devient une intention et une décision morale. Interroger les rapports entre éthique et éthologie, c'est sonder la part de l'involontaire dans nos conduites volontaires, De fait, toute conduite éthique suppose une anthropologie, l'éthologie incitant à reconsidérer l'anthropologie qui fonde notre éthique. « L'animal éducateur de l'homme » donnerait-il les éléments d'une éthique du sensible, correctif nécessaire à une éthique rationnelle?

\section{L'éthologie et les fondements naturels de la morale?}

Si l'éthologie sonde l'archéologie de nos conduites, passer de ce que l'on connaît à ce qui devrait être, n'est ce pas passer d'un jugement de fait à un jugement de valeur? Connaître comment nous agissons ne dit encore rien de ce que nous ferons lorsque nous agirons. Sauf à réduire la vie morale à une stratégie de bonne entente sociale, négligeant la construction d'une intériorité évaluant ses initiatives en termes d'estime et de mésestime de soi et de sollicitude à l'égard d'autrui, l'éthique trouvera dans l'éthologie une condition nécessaire mais non suffisante. L'éthologie ne dispense pas d'avoir à choisir, toute notre dignité résidant précisément dans le pouvoir d'opter, de décider et d'accomplir. Une faute épistémologique serait de glisser de l'éthologie à l'éthique, faisant de l'éthologie une science normative. Témoin en est ce propos, à cheval entre éthologie et neuroéthique, du primatologue et éthologue Frans De Waal :

«La prise de décision morale est mue par les émotions. Elle active des parties de notre cerveau qui remontent au passage de l'état de reptiles à sang froid à celui de mammifères nourrissant leurs petits, attentifs, aimants que nous sommes. Nous sommes équipés d'une boussole interne qui nous indique comment nous devons traiter autrui. Les rationalisations surviennent souvent après coup quand nous avons déjà réagi suivant les prédispositions de notre espèce. Elles nous permettront de justifier nos actes auprès des autres, qui pourront alors les entériner ou non, de façon à ce que la société tout entière parvienne à un consensus sur un dilemme moral précis. C'est là que la pression sociale entre en jeu - l'accord ou le désaccord qui revêtent pour nous une importance décisive -, mais tout ceci passe probablement après la morale "viscérale"12 ».

Outre le coup de force passant du probable au nécessaire, cette thèse fait de la réaction le modèle de l'action, dans un court-circuit de la délibération au profit de l'impulsion qui interroge : peut-on faire, sans dommages, de l'impulsion, l'étiage ordinaire de l'agir? Mais cette place «décisive » donnée aux émotions, estompe le rôle de la délibération morale, c'est-à-dire comment l'accompagnement émotionnel d'une décision est rapporté singulièrement à une situation spécifique et intentionnellement représentée. Si la réactivité suppose des signaux déclencheurs auxquels répondent des comportements adaptés mus par des émotions, l'univers éthique embrasse l'ensemble de ces signaux dans un univers symbolique qui modifie la signification même accordée à ces signaux, pour l'orienter intentionnellement sur une option. L'éthologue veut mettre au jour une sémiotique, là où l'éthique relève d'une sémantique de l'action laissant sa place à l'interprétation. Pourtant, l'éthologue insiste :

«L'empathie est largement répandue chez les animaux... Peut-être l'empathie a-t-elle atteint son apogée dans notre espèce, mais plusieurs autres animaux - et tout spécialement les grands singes, les dauphins et les éléphants - nous suivent de près... Ils ignorent peut-être la règle

\footnotetext{
${ }^{12}$ De Waal, 2006, pp. 240-241.
} 
d'or, mais ils semblent indiscutablement s'y conformer [...] Sans émotions, c'est à peine si nous saurions effectuer des choix de vie, car ils se fondent sur des préférences et, en dernier ressort, les préférences sont émotionnelles... Sans émotions nous resterions indifférents aux autres qui nous rendraient la pareille. [...] Nous glorifions la rationalité, mais au moment critique où elle devrait se faire entendre, nous la traitons en quantité négligeable. [...] la morale humaine est solidement ancrée dans les émotions sociales, l'empathie se situant à son cœur. Les émotions nous servent de boussole ${ }^{13} »$.

Est-ce entre la raison combinatoire de l'automate informatique et l'émotion de l'animal, qu'il faut-il choisir, comme le pense l'éthologue ? Mais le syllogisme pratique ne se réduit pas à une déduction formelle, abstraite et froide. La constance morale n'est pas la cohérence logique, la vie morale se jaugeant à l'aune d'une trajectoire de vie (biographie) et non à la ponctualité d'une impulsion. Dénoncer l'incapacité de la logique à mobiliser vraiment pour agir pour en conclure que le vrai moteur est l'empathie, est un coup de force ignorant la dimension narrative attachée à l'identité du sujet moral et la nature de la sagesse pratique. L'empathie présente en l'animal, - celle qui faisait dire à Rousseau : « dans le Dictionnaire de Botanique, je vois un sentiment exquis dans mon chien, mais je n'en aperçois aucun dans un chou »-est une réaction sensible de répugnance, mais non encore un sens moral. De fait, la réduction éthologique de l'éthique méconnaît combien celle-ci est essentiellement une manière de coordonner, dans une visée de la vie bonne et en un monde, ce que l'éthologue analyse, décompose, isole et atomise en " éléments simples » : l'empathie, la responsabilité, la solidarité, etc.

Asseoir la règle morale dans la nature n'est pas sans excès. Si l'empathie dont fait preuve l'animal indique en lui, comme en nous, une dilatation de la sensibilité capable d'aller jusqu'à la souffrance de l'autre - l'aversion du cheval à piétiner un corps vivant tombé à terre -, cette répugnance n'est pas la compassion, pas plus que l'effroi n'est le sens de la dignité. L'empathie animale est présence sensible devant le spectacle répugnant de la douleur, là où le sens moral est une représentation ouverte au « sens » de la souffrance de l'autre. C'est ce qui expliquera que la responsabilité morale, exercée en faveur de l'animal, puisse être une responsabilité sans réciprocité ! Incontestablement, l'éthologie donne l'occasion de faire sa juste part à la dimension infra-rationnelle et émotionnelle de la vie morale, révélant sa part sensible à notre sens moral, mais celui-ci n'est pas celle-là. Qu'il y ait une boussole interne - on peut ici prendre acte des découvertes liées aux sciences du cerveau, notamment les techniques de neuro-imageries comparant cerveau humain/cerveau de primate -, n'autorise pas à en faire un sens moral. Indiquer une direction ne signifie pas qu'on la prenne, la douloureuse histoire du mal que l'homme fait à l'homme le rappelant. Ainsi, la partie activée du cerveau que révèle la neuro-imagerie n'explicite pas le contenu d'un jugement moral singulier mais rend « visible » l'opération d'un jugement moral, sollicitant toujours la même zone cérébrale. L'expression neuronale de l'équité ne dit rien encore de la visée singulière de ce qui est équitable dans une situation effective. La pathologie le révèle : la zone cérébrale de la joie peut être activée, révéler l'expression de la joie, mais l'exprimant « sans raisons ». Être «neuronalement» joyeux, mais sans raisons, est-ce être joyeux ? Par ailleurs, la variabilité des images de cerveau d'un individu à l'autre, et pour un même individu, est si grande, que le contenu intentionnel d'un jugement moral est loin d'être totalement

\footnotetext{
${ }^{13}$ De Waal, 2006, pp. 236-238.
} 
certifié (Jeannerod, 2005). Aussi, la comparaison entre l'image corticale du primate riant et celle d'un humain faisant de même suggère, sans le démontrer vraiment, qu'il y a là une identité d'expérience. Savoir que quelque chose a lieu qui a quelque semblance n'est pas savoir ce qui a eu effectivement lieu. Le propos de Frans De Waal, ignore enfin combien entre l'émotion et la rationalisation s'opère tout un travail de schématisation pratique, révélant le rôle actif d'une imagination morale. Être réactif suppose le travail d'un sens moral assis sur le rôle schématisant d'une imagination pratique, laquelle condense en images des options normatives et des émotions reptiliennes, sur lesquelles il n'est pas encore possible d'avoir une juste idée, mais avec lesquelles se mobiliser en vue d'agir. Dans l'action urgente, le pressentiment de son importance tient à ce qu'on en imagine les conséquences plus qu'on ne les sait.

Ces réserves faites, le concept éthologique de schème d'actions est éclairant pour l'éthique. Il «...souligne la constance relative de quelques activités motrices complexes, observables, sans nécessairement sous-entendre une spécificité ou universalité d'espèce, ni supposer quoi que ce soit concernant l'ontogenèse du comportement (Stroobants in Morris, 1978, p. 398). » Le schème d'action souligne les composantes originaires et élémentaires œuvrant dans les conduites humaines, dans un continuum conduisant de l'involontaire au volontaire supposant sa reprise réflexive. Tels sont du côté de la nature, les automatismes, les déclencheurs comportementaux, les conduites « machinales », et du côté de l'histoire les conduites porteuses d'une certaine stéréotypie, le vaste champ des habitudes et de la répétition. Dans Le volontaire et l'involontaire, Ricœur distingue mouvements instinctifs et conduites préformées (Ricœur, 1950, ch. 2, pp. 216 sq.), clarifiant le rôle actif que jouent les mœurs, comprises comme habitudes de vie, dans la vie morale. Ni instinct, ni réflexe, les mœurs sont une forme d'involontaire en nous, dont la manifestation demande dans quelle mesure «y sommesnous pour quelque chose »? Du point de vue de l'histoire et de la liberté, les bonnes mœurs, les conduites convenues ou convenables sont parfois tenues analogiquement pour machinales ou réflexes, préformant nos conduites, mais ce ne sont pas des involontaires absolus, puisque, sur elles, une forme d'initiative est possible pour peu qu'on le veuille. Une conduite préformée échappe au concept de comportement, surdéterminé par sa réification behavioriste. La conduite suppose une organisation et une finalité qui la distinguent des mouvements produits « en nous sans nous par une action des choses sur nous » (Ricœur, 1950, p. 218). Du point de vue de la nature, le terme préformé est le point d'articulation entre éthologie et éthique, observant que nous ne sommes pas totalement à l'initiative de nos schèmes d'actions. Il y a là une forme de solidarité de l'agent avec le monde, telle que venir au monde signifie venir en un monde préformé, porteur de «déclencheurs comportementaux» (Lorenz), de schèmes moteurs. Une conduite préformée, dans sa signification pratique, laisse entendre la possibilité d'une continuité théorique entre schèmes moteurs présents dans les comportements animaux (le regard soutenu comme signe de l'agression) et les pré-compréhensions pratiques qui investissent les conduites humaines (le regard interprété comme signe d'une insolence). Le monde humain, entendu comme univers symbolique, est la reprise dans le champ des médiations pratiques, de ces schèmes moteurs et des déclencheurs comportementaux. Les conduites préformées, intégrées dans un monde de signes, sont métamorphosées en pré-compréhensions pratiques grâce auxquelles préciser ce que signifie se bien conduire. 
Forme de disposition régulière proche des schèmes d'action, les mœurs sont des réalités mixtes, occupant le point frontière entre les schèmes moteurs des comportements animaux en leur limite inférieure et les pré-compréhensions pratiques en leur limite supérieure. De ce fait, elles ont une présence structurante et différenciante. Opérationnelles, sur le plan de la motricité corporelle, mais aussi sur celui des conduites socioculturelles, les mœurs, plus que le résidu de l'animalité en nous, profilent une façon de penser et d'organiser ce qu'exister en homme veut dire. «Habitualité » du côté de la nature, les mœurs assument du côté de la culture, la façon que nous avons d'être traversés par le temps que nous traversons. Mixte de nature et d'histoire, quoique sur des seuils différents, les mœurs humaines ou animales opèrent selon un principe d'économie, les initiatives prises étant relayées par des dispositifs corporels dispensant d'avoir à se disposer à nouveau. Mais la conduite humaine n'interdit pas que l'initiative soit de nouveau reprise sur les mœurs, depuis les formes motrices jusqu'aux pré-compréhensions pratiques qu'elles constituent. Ainsi les formes motrices, ayant pris leur indépendance à l'égard des apprentissages moteurs de mouvements segmentés ou analytiques, deviennent-elles des relais efficaces pour apprendre ou exécuter des suites motrices apparentées. De même, les pré-compréhensions pratiques que sont les mœurs jouent-elles en nous comme une manière d'actions particulières : elles sont agissantes sans être véritablement des actions.

L'opposition comportement animal/conduite humaine révèle la difficulté qu'il y a à décrire méthodiquement en termes de seuils ce qui ontologiquement repose sur une différence d'essence. Le milieu des mœurs animales s'explicite en des comportements adaptés, là où le monde des conduites humaines se fait significations à discuter, d'où des ratés, des hésitations, ou des réussites pensées en termes d'estime de soi. Un milieu de signaux préformant des comportements n'est pas un univers symbolique ouvrant sur des conduites dont la signification est à interpréter. C'est pourquoi l'agir humain, dans les mœurs qui sont autant nature qu'histoire, n'est pas prisonnier de stéréotypes définitivement figés. La conscience, dans les mœurs mises en œuvre, n'est pas abolie, mais est seulement suspendue. Les mœurs sont la naturalisation utile de la morale. Entendons par là qu'elles jouent comme un principe d'économie qui dispose à l'essentielle attention en dispensant d'avoir à se conduire dans l'ordinaire fonctionnalité de bien des situations où nous nous trouvons. Certes les mœurs peuvent dégénérer en un réseau de conduites sédimentées. C'est là une tentation, non une fatalité. Mais si l'ethos n'est pas le tout de l'éthique, il se souvient que la vie morale s'évalue certes dans le moment ponctuel de la décision, mais également dans sa dimension narrative et historique.

\section{La comédie des mœurs : éthologie et imaginaire de l'action}

La critique des mœurs humaines par les mœurs animales a trouvé en Jean de La Fontaine un moraliste incontestable. Nous passons alors des mœurs analysées en leur perspective génétique (de l'animal à l'homme), aux mœurs convoquées comme un outil généalogique (de l'animal à l'animal humain) Ici, l'éthologie y est élevée au rang d'instrument de veille éthique. L'imaginaire animalier de la fable, plus qu'un procédé rhétorique servant une critique des mœurs de Cour confondant l'éthique et l'étiquette, 
invente un lien imagé entre une qualité ou un travers humain et la consistance singulière d'un comportement animal (le rusé renard.)

Défenseur de l'animal contre la pensée mécaniste, La Fontaine est aussi un philosophe attentif à la composante éthologique de la vie éthique, ménageant une analogie fonctionnelle entre la conduite humaine et le comportement animal (jalousie, ruse, vanité...), cultivant une manière de schématisme pratique à propos de « mœurs semblables ». Lire ainsi La Fontaine, c'est reconnaître que les éthologues sont aujourd'hui nos nouveaux moralistes ${ }^{14}$. Le spectacle des mœurs animales est un excellent rempart contre la comédie des mœurs humaines. Contre la vanité des hommes, pas de meilleure critique que la simplicité native des comportements animaliers. En ce sens, la pédagogie des fables, outre le statut d'animalisation d'une vertu rendant compréhensible son abstraction, prend la mesure d'invariants actifs dans les mœurs animales opérant dans les mœurs humaines, écartant la tentation vaniteuse d'enfermer l'homme dans un splendide isolement. Dans son Discours à Madame de la Sablière ${ }^{15}$, La Fontaine critique le mécanisme cartésien comparant l'animal à une montre, à des fins éthiques. - «Qu'est-ce donc ? - Une montre. - Et nous ? - C'est autre chose ». La force ironique du « Et nous ? » rappelle que la modélisation de l'animal n'est jamais éthiquement neutre. Penser l'animal comme une machine force à chercher le machinal, sinon le machinique en l'homme. Inversement, découvrir l'animal comme capable d'innovation comportementale (le cerf ou le castor dont parle La Fontaine dans le Discours), c'est chercher la base éthologique de la visée éthique. Lire les éthologues comme des moralistes, rend alors attentif à la statique comportementale qui donne son assise à la dynamique des conduites humaines. L'éthologie comparée fait plus qu'illustrer une «morale. » Elle invite à mesurer l'impact de la disposition éthologique active dans la vie morale. Mais, plus encore, les mœurs et les images animalières investissent le champ de l'analyse, de l'élucidation, de l'évaluation, de la décision et de la vie morale. Les figures animalières opèrent comme des relais imagés rendant sensibles des options axiologiques. Outre l'animal utilisé comme allégorie (le lion et la bravoure, le serpent et la séduction, l'âne et la bêtise) dans les bestiaires, l'effet distanciant de l'image animale rend sensible des traits de conduites et exerce une propédeutique morale. Tel est l'animal convoqué au titre d'exempla, donnant à voir des comportements exemplaires ou édifiants (l'abeille ou la fourmi industrieuse, etc.). L'animal pensé et rêvé, l'animal imaginé, véritable analogon, sert alors les imaginaires politiques - l'imaginaire congelé de l'image faite idole dans l'utopie de la société parfaite ( $c f$. l'image idolâtrée de la société parfaitement ordonnée dans l'imaginaire de la termitière qui fascine tant la sociobiologie) et les imaginaires moraux ( $c f$. l'image de l'animal comme figure du sacrifice moral avec le pélican) ou religieux (le serviteur souffrant et l'imaginaire de l'agneau immolé dans le christianisme). Enfin, la fonction prospective d'une imagination créatrice trouve dans les mœurs animales une poétique de l'action nourrissant des analogies fructueuses pour imaginer d'autres formes de solidarités ou d'initiatives possibles. On pense à cette fraternité élargie du sensible avec le sensible dans la symbiose que préfigure le chant fraternel de François d'Assise à son « frère loup ».

\footnotetext{
${ }^{14}$ Sur ce point, titres et intertitres de l'ouvrage de Frans De Waal, 2006, parlent d'eux-mêmes. ch. 2, « Pouvoir : du Machiavel dans notre sang », chap. 3, «Sexes : les primates du Kamasoutra », ch. 4, «Violence : de la guerre à la paix $\gg .$. .

${ }^{15}$ Fables, fin du livre IX, vers 24 à 178.
} 
Toute éthique convoque une anthropologie. L'éthologie impose de rompre avec l'anthropologie du dualisme âme/corps, au profit d'une anthropologie attentive à l'incarnation sensible de l'identité morale (depuis l'appareillage neuronal jusqu'au rôle des émotions et des schèmes moteurs présents chez l'animal et sur lesquels s'élabore l'agir). Il s'ensuit que l'éthique retrouve, avec l'éthologie, la force des mobiles sensibles. Émotions et sensibilité, sans céder à la sensiblerie, en découvrant leur part mobilisatrice dans les décisions, élargissent le champ de nos obligations et de nos responsabilités à des fraternités plus vastes avec les vivants. Si l'éthologie peut être un élément pour une éthique faisant sa part à la sensibilité dans la vie morale, l'émotion est un fait moral originaire signalant la capillarité secrète de la vie qui reconnaît la vie. En troisième lieu, l'éthologie libère l'éthique d'un humanisme narcissique sans céder à la tentation d'un antihumanisme biocentrique. L'animal éducateur de l'homme invite à une leçon d'humilité, non à son humiliation. Le souci de l'animal n'est pas exclusif de la sollicitude manifestée à l'égard de l'homme. Il s'inaugure ainsi un humanisme fragile mais non faible, montrant qu'humanisme et écologie peuvent faire bon ménage ailleurs que dans les ménageries. Une forme de solidarité universelle est possible et pensable lorsque l'homme se souvient de cette part de l'autre en lui que l'animal décline devant ses yeux. En quatrième lieu, une fraternité reconnue avec l'animal n'est pas incompatible avec l'affirmation d'une différence anthropologique qui est moins celle d'une supériorité, que la réjouissance métaphysique qu'engendre tout être irremplaçable. Parler de différence anthropologique n'est pas une offense faite aux animaux, mais l'attestation d'un être au monde original lui donnant des obligations spécifiques à l'égard de l'animal. Les liens entre éthique et éthologie ne sont donc pas sans conséquences quant à la responsabilité à l'égard de l'animal. Si l'animal n'est pas un quelqu'un, il n'est pas réductible à un quelque chose. Il s'ensuit que nous prenons là la mesure, inspirée par Hans Jonas, d'une responsabilité d'un nouveau type, d'une responsabilité à l'égard du caractère périssable du vivant (biodiversité), mais qui est une responsabilité sans réciprocité définie. Ni objet, ni sujet, l'animal est un être. La communauté sensible qui nous lie à tout être vulnérable justifie moralement qu'on ne puisse traiter les animaux comme des choses, et explicite la revendication, sinon d'un droit propre des animaux, du moins d'un droit pour les animaux. Les hommes portent unilatéralement les obligations envers des animaux non sujets de droit (ex. : leur maltraitance inutile). Mais ces obligations pré-rationnelles, non artificiellement et positivement fondées, tiennent au fait que ne pas honorer le sensible en soi et en dehors de soi, revient à déshonorer sa propre humanité. Traiter humainement un animal n'est pas faire de l'animal un humain mais reconnaître que la grandeur de notre humanité se tient aussi dans la dignité sensible de nos conduites. Enfin, si l'éthologie découvre l'importance de schèmes d'actions préformés dans l'agir, ces derniers ne font pas à eux seuls une action. La liberté de l'agir humain se trouve précisément concentrée dans la rencontre entre des dispositifs préparant l'agir et la disposition singulière à agir dans une circonstance reconnue comme inédite. Si l'éthologie envisage le problème de l'agir comme résolu, l'éthique le maintient comme un problème à résoudre. Dans l'action, l'événement reste notre maître; c'est là toute notre liberté. 



\section{Chapitre 11 \\ Les propositions d'extraction des animaux de la catégorie des objets de droit}

SONIA DESMOULIN

Les partisans d'une évolution de la condition juridique des animaux ne prônent pas tous le même remède. S'ils considèrent que les informations relatives à la vie sociale, à l'intelligence ou à la sensibilité animale sont déterminantes, leurs positions divergent concernant la solution juridique adéquate pour en tenir compte. D'aucuns estiment que l'animal doit être intégré dans la catégorie des sujets de droit. D'autres appellent à revoir la summa divisio entre personnes et choses où l'animal ne trouverait pas sa place. Cette dernière proposition traduit le souci de reconnaître à l'animal un statut original en droit, le distinguant des « biens de consommation » sans pour autant le transformer en personnage de la scène juridique. Elle est en réalité bien difficile à admettre tant elle bouleverse la structure de notre droit. Le projet de création d'une personnalité juridique animale, quant à lui, remet en question nombre de postulats de notre système juridique. Le droit a pour fonction d'organiser et de régir les activités humaines. L'homme est à la fois l'acteur et la préoccupation première de l'ordre juridique et de la procédure judiciaire. Revenir sur ces principes conduirait à modifier gravement l'institution juridique qu'est la personne. Si le recours à la fiction juridique permet de faire abstraction de certaines réalités, cet outil a des limites. Il est des évidences que le pouvoir créateur du droit ne saurait impunément négliger, surtout si l'utilité de l'artifice apparaît douteuse. Or, la personnification juridique de l'animal se révèle être, à l'analyse, une fiction inopportune.

\section{Diversité des propositions d'extraction de l'animal de la catégorie des choses}

L'idée d'attribuer à l'animal un statut juridique différent de celui des choses est formulée dès le début du $\mathrm{XX}^{\mathrm{e}}$ siècle, en liaison avec les conclusions darwiniennes et les 
premiers travaux scientifiques sur les sociétés animales. Elle prend cependant un tour différent selon que l'on intègre ou non les animaux dans la catégorie des personnes.

\section{L'animal, intermédiaire entre les personnes et les choses}

La proposition de créer une tierce catégorie, entre les personnes et les choses, pour refléter la spécificité animale fut exprimée à l'aube du Xx ${ }^{\mathrm{e}}$ siècle par Paul Janet. Celui-ci écrivait que les animaux devaient bénéficier d' « une place à part au-dessous de l'humanité, et que, titulaires de demi-droits, ils étaient des demi-personnes » (Giberne, 1931, p. 92). On retrouve l'idée chez des auteurs contemporains : certains appelant à la réforme du droit positif, d'autres affirmant que cette évolution est déjà réalisée (Iacub, 2005, pp. 31 sq.). Parmi les propositions les plus abouties, on relèvera celle de Françoise Ringel et d'Emmanuel Putman. Constatant que «l'animal [...] s'impose à nous comme être animé, sensible et sociable », les auteurs concluent qu' " entre personne et chose, sujet et objet, il est peut-être temps d'admettre la possibilité d'une notion intermédiaire » (Ringel et Putman, 1995, p. 45, cit., pp. 46-47). Afin de renforcer la protection des animaux, c'est-à-dire essentiellement des animaux domestiques et surtout des animaux de compagnie, ils proposent de créer « une catégorie juridique sui generis » (Ringel et Putman, 1995, p. 54). Il s'agirait d'une forme de «protection par l'incapacité » permettant à l'animal « dénué de vraie personnalité juridique » (Ringel et Putman, 1995, p. 57) d'être représenté en justice aux plans civil et pénal non seulement par les associations agréées mais par «tout intéressé » (Ringel et Putman, 1995, p. 58). On peut objecter qu'il n'est nul besoin de bouleverser l'organisation multi-séculaire de notre droit, en abandonnant la dichotomie entre personne et chose, pour admettre plus largement l'action des associations en matières civile et pénale. Quant à l'ouverture du prétoire à « tout intéressé », elle pose naturellement le problème des actions vindicatives et de l'encombrement des tribunaux.

S'agissant de la première objection, des auteurs contestent les " arrière-pensées non juridiques » qui soutiendraient «la "thèse" d'une soi-disant summa divisio juridique des personnes et des choses » (Farjat, 2002, Art., p. 221, cit., p. 222). Cette thèse serait erronée au regard notamment des réalités économiques. Aussi, M. Gérard Farjat proposet-il de reconnaître l'existence de « centres d'intérêts » qui ne seraient ni des personnes ni des choses. À des fins pratiques, la famille, les groupes de sociétés, l'entreprise seraient reconnus comme des «succédanés de personnes morales » (Farjat, 2002, Art. p. 221, cit., p. 222), tandis que les animaux figureraient, avec les embryons, parmi les « ombres de personnes physiques » (Farjat, 2002, pp. 228). La proposition n'a donc pas pour seul objet de tenir compte de la spécificité animale, mais cherche à valoriser le réalisme en droit. Séduisante, la démarche recèle pourtant maintes difficultés. Ainsi, la définition de ces « centres d'intérêt » et la systématisation de leur régime juridique posent problème. Il faudrait donc abandonner un système dont la logique et la fonctionnalité ont fait leur preuve, pour lui substituer un système inabouti dont on cerne difficilement la logique et le fonctionnement. La summa divisio entre les personnes et les choses traduit l'opposition fondamentale entre les sujets de droits et d'obligations qui animent le droit et les causes ou les objets à propos desquels peuvent survenir des conflits entre personnes. Que seraient ces « centres d'intérêt » qui ne seraient ni acteurs ni objets du théâtre judiciaire?

On comprend que l'idée de catégorie intermédiaire rencontre des critiques. Concevoir cette troisième catégorie est fort difficile. La pertinence d'une telle innovation n'apparaît 
pas clairement. Non seulement la distinction entre les personnes et les choses est historiquement fondatrice de notre droit, mais l'évolution ne manquerait pas de créer des perturbations méthodologiques, car le juriste procède par intégration ou exclusion, l'exclusion impliquant le recours à la qualification générale ou par défaut. Plus fondamentalement, la dichotomie reflète la logique profonde du système juridique. En tant qu'instrument d'ordre et de paix sociale, en tant qu'art de la recherche de solutions justes, le droit exige que des acteurs lui donnent vie. Les sujets de droit sont indispensables à son existence. Toutes les autres entités susceptibles d'intéresser le droit mais incapables de participer à sa mise en scène sont logiquement qualifiés d'objets. Certes, l'animal présente des traits remarquables, et l'on a pu dire qu'au sein des entités saisies par le droit il est la «plus proche de l'homme » (Carbonnier, 2004, n 887). Certes, parmi toutes les spécificités que les objets du droit peuvent présenter, la sensibilité est d'une pertinence particulière car l'homme moral lui attribue une réelle importance. Toutefois, cette sensibilité ne fait pas de l'animal un acteur de la vie juridique. Elle peut en faire un objet ${ }^{1}$ particulier. Nous partageons le scepticisme du Recteur Claude Lombois quant à l'intérêt d'une qualification qui reviendrait à reconnaître l'existence juridique d'êtres qui « pourraient être pratiquement traités comme des choses après génuflexion devant l'affirmation de principe qu'ils n'en sont pas. [...] Ce serait une catégorie bâtarde où se dissocieraient nature et régime juridique, la première regardant vers le haut et le second, vers le bas » (Lombois, in Marguénaud, 1992, p. II). Bouleverser le système juridique actuel en abandonnant la distinction entre les personnes et les choses, les sujets et les objets, susciterait bien des difficultés pour un résultat d'un intérêt discutable.

Qu'en est-il du raisonnement selon lequel les animaux ne pouvant plus être des choses ou des objets devraient d'ores et déjà être considérés comme des personnes ou des sujets?

\section{L'animal, sujet de droits}

Le raisonnement tenu par de nombreux partisans de la cause animale pourrait se résumer ainsi : la science nous apprend que l'animal est un être sensible et la loi doit consacrer cette réalité; cette réalité légalement consacrée implique une adaptation du régime juridique et, à sa suite, un changement de nature juridique ainsi qu'une modification des mots du droit, porteurs de leur poids de symbole.

Avec l'accumulation des données scientifiques la question de la nature juridique de l'animal se pose donc avec une acuité sans précédent ${ }^{2}$. Cependant, le propos n'est pas nouveau. Dans un ouvrage publié en 1900, Edouard Engelhardt écrivait déjà : «Peut-on se refuser à croire qu'avec les certitudes nouvelles dont la science contemporaine s'enrichit tous les jours, le sentiment de plus en plus intime de ces affinités et de la solidarité qu'elles impliquent, portera la créature qui est "la plus haute expression de la vie" à associer plus directement à ses destinées, du moins ceux qui se rapprochent le plus d'elle et sont en majeure partie ses auxiliaires indispensables ? (Engelhardt, 1900, p. 4). Le juriste invite donc à élaborer un nouveau statut juridique pour l'animal et propose de

\footnotetext{
${ }^{1}$ C'est-à-dire tout à la fois une entité concrète, sur quoi porte une réflexion et des sentiments, et le but des règles visant à le protéger.

${ }^{2}$ En ce sens, $c f$. Terré et Fenouillet, 2005, n 13.
} 
considérer les animaux comme des sujets de droits naturels auxquels le droit positif attribuerait « une "parcelle", un analogon de la personnalité humaine ».

Renouvelant le propos, des philosophes soutiennent aujourd'hui que les animaux doivent être reconnus comme sujets de droit, ce qui implique sur le terrain du droit positif de leur conférer la personnalité juridique. Georges Chapoutier est ainsi convaincu que les animaux présentent « des caractères partiels d'une personne - y compris, pour les animaux "supérieurs" des caractéristiques d'intelligence et d'autoconscience analogues » à celles de l'homme : langage, sentiment du temps... « Les animaux peuvent donc avoir des droits, même si, bien entendu - et il faut [...] insister sur ce point -, ceux-ci diffèrent

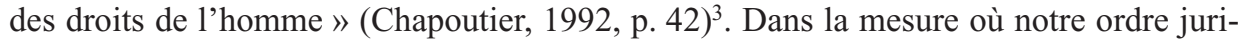
dique impose le recours à la personnalité juridique pour l'attribution de droits, le philosophe se prononce pour la reconnaissance d'une «forme de personnalité juridique », une "personne animale particulière » différente des personnes juridiques existantes (Chapoutier, 1992, p. 50).

Ces idées ont trouvé leur expression la plus marquante dans la rédaction d'une Déclaration universelle des droits de $l^{\prime}$ animal ${ }^{4}$, qui sert de référence à nombre de défenseurs de la cause animale. Le préambule de cette Déclaration débute par l'affirmation que « la Vie est une, tous les êtres vivants s'étant différenciés au cours de l'évolution des espèces », que « tout être vivant possède des droits naturels et que tout animal doté d'un système nerveux possède des droits particuliers ». Une série d'articles affirme ensuite l'existence de droits pour les animaux ${ }^{5}$. Les animaux ont ainsi « des droits égaux à l'existence dans le cadre des équilibres biologiques », selon l'article $1^{\mathrm{er}}$, alinéa $1^{\mathrm{er}}$. L'article 9 précise que « la personnalité juridique de l'animal et ses droits doivent être reconnus par la loi ».

Cette Déclaration semble relever de l'influence de la philosophie des droits de l'animal, actuellement défendue par Tom Regan. Ce dernier considère en effet que les animaux conscients, y compris l'homme, sont des «sujets de leur vie » et donc des sujets de droits (Regan, 1983) ${ }^{6}$. Cependant, la Déclaration semble également puiser à la source de la philosophie utilitariste, bien que celle-ci se distingue de la philosophie des droits. On trouve ainsi sous la plume du président de la Ligue française des droits de l'animal cette double affirmation : « cette déclaration vise à établir une égalité de droits à la vie, quelle que soit l'espèce animale, espèce humaine comprise »; " si elle exclut la cruauté de l'homme, et l'utilisation abusive qu'il fait de l'animal, elle vise surtout à exclure le spécisme, dû aux hiérarchies que l'homme a établies à son propre usage, et selon des critères anthropomorphiques », car « de telles hiérarchies sont totalement arbitraires, spécistes, et ne correspondent à aucune réalité scientifique. » (Nouët, in Couret et Oge, 1988, p. 393). La dénonciation du spécisme et de l'absence de scientificité des

\footnotetext{
${ }^{3}$ En ce sens, $c f$., également, Castignone, 1988, p. 325.

${ }^{4}$ Inspirée des travaux de Georges Heuse et proclamée le 15 octobre 1978 à la Maison de l'UNESCO, la Déclaration est l'œuvre du travail conjugué de plusieurs associations de défense des animaux et de personnalités sensibles à cette question. Elle a été modifiée par la L.F.D.A. en 1989, qui lui a adjoint le préambule et l'annexe présentés. Elle est reproduite in : Antoine et. Nouët, 2003, pp. 23 sq.

5 «Toute vie animale a droit au respect» (art. 2). L'animal sauvage « a le droit de vivre libre dans son milieu naturel et de s'y reproduire » (art. 4). « L'animal mort doit être traité avec décence » (art. 3, alinéa 3). « L'animal que 1'homme tient sous sa dépendance a droit à un entretien et à des soins attentifs » (art. 5, alinéa $1^{\mathrm{er}}$ ).

${ }^{6}$ Pour une présentation rapide et claire des thèses de T. Regan, on pourra consulter Goffi, 1994, pp. 193 sq.
} 
arguments relève clairement de la rhétorique utilitariste développée par Peter Singer (1993).

Reposant sur le «principe du plus grand bonheur », l'utilitarisme postule que nos actions doivent être déterminées à l'aune de leurs conséquences positives ou négatives. Nos actions doivent être utiles, c'est-à-dire « produire bénéfice, avantage, plaisir, bien ou bonheur » ou " prévenir l'apparition de dommage, douleur, mal ou malheur, en la partie dont l'intérêt est envisagé "), selon la formule de Jeremy Bentham (Bentham, in Burns et Hart, 1970, p. 12). Or, si l'on admet que les animaux ne sont pas de simples mécanismes mus par d'infimes rouages (version cartésienne) ou par des réflexes en réponse à des stimuli (version pavlovienne ou behavioriste), mais des êtres sensibles, il faut en tirer les conséquences au regard de nos actions.

Sur ces fondements, Peter Singer a développé une théorie de la « libération animale » (Singer, 1993. Cf., également, Cavalieri, 1991). La spécificité de sa doctrine réside dans la distinction à opérer entre les animaux simplement conscients, c'est-à-dire capables d'éprouver plaisir et souffrance, et ceux qui ont conscience d'eux-mêmes, c'est-à-dire qui ont une vie mentale assez complexe pour former des préférences et chercher à les satisfaire. Peter Singer pense en termes philosophiques et éthiques. En théorie, il se désintéresse de la réflexion juridique, considérant que « l'emploi du vocabulaire des droits ne représente qu'un raccourci politique pratique » (Singer, 1993, p. 17) ${ }^{7}$. Cependant, dans un « Projet grands singes » (1994) co-dirigé avec Paola Cavalieri, il utilise la rhétorique des droits pour contester la réservation des droits de l'homme aux êtres humains. Le Great Ape Project entend faire bénéficier les quatre espèces de grands singes de la protection accordée aux personnes humaines, notamment dans le cadre de l'expérimentation biomédicale. Au regard des propositions formulées et du vocabulaire utilisé, ce « projet» est destiné à trouver une traduction juridique. Il s'agit donc bien d'une thèse visant à faire de certains animaux des sujets de droit.

La notion de sensibilité est au cœur des thèses utilitaristes et se retrouve au fondement de la plupart des discours pour l'évolution de la condition juridique de l'animal. Selon les utilitaristes, la souffrance ou le plaisir ressenti par l'animal doivent entrer dans le calcul de maximisation du plaisir. Selon les partisans des droits des animaux, la sensibilité désigne l'existence d'un «vouloir vivre », donc d'un individu qui a une valeur intrinsèque. Dans le domaine de la théorie juridique, la prise en considération de cette sensibilité s'exprime par le biais du concept d'intérêt, concept fondateur pour les propositions visant à conférer une personnalité juridique aux animaux.

Lorsque Caroline Daigueperse appelle à la consécration d'un nouveau statut juridique pour l'animal, elle développe sa réflexion autour du concept d' « intérêts juridiquement protégés ». Elle affirme ainsi que «les droits servent à garantir les intérêts de la vie, à aider à ses besoins, à réaliser ses buts » et que " des droits subjectifs, en tant "qu'intérêts juridiquement protégés" peuvent être reconnus à l'animal» (Daigueperse, 1981, Doc., p. 160, cit., p. 163). Cette évolution s'impose à ses yeux car «tant que les législations nationales n'auront pas explicitement admis et reconnus les droits de l'animal, la protection de ce dernier ne sera qu'imparfaite » (Daigueperse, 1981, p. 160). Pour la réaliser,

\footnotetext{
${ }^{7}$ On ne peut contester cette volonté déclarée de limiter ses propositions au champ philosophique ou éthique sans prendre le risque de se confronter ainsi aux difficultés d'une tradition effective de ses principes dans la réalité juridique.
} 
la juriste propose de considérer l'animal comme « un sujet de droit d'un type particulier ». « On peut fort bien envisager », écrit-elle, « même par une construction technique, [qu']au même titre que les personnes morales, les animaux aient des droits consacrés par la loi » (Daigueperse, 1981, p. 163 ; Brunois, 1984).

Cette proposition n'est guère éloignée de la thèse de Jean-Pierre Marguénaud (1992, pp. 387 sq.) visant à consacrer une personnalité juridique animale ${ }^{8}$. S'appuyant sur les recherches menées sur le comportement animal, notamment dans les «sociétés de vertébrés », l'auteur avance que certains animaux seraient «conscients du droit» (Marguénaud, 1992, pp. 366 sq. ; Sacco, 1995, p. 783). Surtout, l'auteur part du constat - qui est en réalité déjà une construction - que l'animal possède désormais en droit positif un « intérêt propre juridiquement protégé ». L'animal étant un être sensible, son intérêt propre résiderait dans la protection contre les souffrances ou une mort prématurée. Pour démontrer que cet «intérêt propre » est «juridiquement protégé », Jean-Pierre Marguénaud relève la succession des lois de protection des animaux. Il estime que, depuis la disparition de la condition de publicité initialement exigée pour la répression des mauvais traitements à animaux, « l'animal est protégé pour lui-même » (Marguénaud, 1998, p. 208). Selon lui, « dans la mesure où il est protégé pour lui-même y compris contre son propriétaire, l'animal n'est plus une chose appropriée », car « une chose appropriée est soumise aux énergiques prérogatives qui découlent de l'art. 544 c. civ. » (Marguénaud, 1998, p. 208) ${ }^{9}$. Or, il constate que le droit d'user librement de son bien est limité par l'interdiction de commettre des actes de cruauté, des sévices graves ou des mauvais traitements sur l'animal ; tandis que l'incrimination de l'abandon et de l'atteinte volontaire à la vie d'un animal en l'absence de toute nécessité attenterait de façon déterminante au pouvoir de disposer. À ces éléments, Jean-Pierre Marguénaud (1998, p. 211) ajoute que tout devoir révèle l'existence d'un droit corrélatif ${ }^{10}$. En conclusion, l'animal ne pourrait plus être qualifié de bien. L'auteur voit d'ailleurs dans les réformes du Code pénal, en 1994, et du Code civil, en 1999, les signes de ce changement de condition juridique $^{11}$. En effet, le législateur a sorti les atteintes aux animaux du livre du Code pénal sur les atteintes aux biens pour les intégrer à un nouveau livre intitulé Des autres crimes et délits. Quant aux articles 524 et 528 du Code civil, leur nouvelle rédaction distingue les animaux des « objets » placés sur un fonds immobilier ou des « corps » qui peuvent se transporter par eux-mêmes.

La démonstration ne convainc guère. En premier lieu, l'assertion selon laquelle tout devoir révèle un droit corrélatif n'est pas vérifiée en droit. Nombre de dispositions pénales ayant pour objet de prévenir des comportements dangereux créent des devoirs mais aucun droit subjectif: les textes contre l'ivresse publique ou ceux encadrant le port d'arme en sont des exemples. En deuxième lieu, le rôle démonstratif conféré aux évolutions du droit pénal laisse dubitatif. Le droit pénal vise, en effet, à protéger non des personnes juridiques mais des êtres réels. Il lutte contre les atteintes portées aux individus et à la société, mais ne permet pas de découvrir les arcanes du système juridique,

\footnotetext{
${ }^{8}$ Voir, également, Marguénaud, 1998, p. 205.

${ }^{9}$ L'article 544 du Code civil dispose : « La propriété est le droit de jouir et disposer des choses de la manière la plus absolue, pourvu qu'on n'en fasse pas un usage prohibé par les lois ou par les règlements. »

${ }^{10}$ Cette idée est notamment développée par André Comte-Sponville, 1995, p. 140.

${ }^{11}$ Marguénaud, 1995, p. 187 ; Marguénaud, 1998, p. 209. Dans le même sens, Antoine, 1996, Chron., p. 126 ; Danti-Juan, 1996, p. 477 ; Bergel et al., 2000, n 453.
} 
ses catégories et ses concepts fondateurs. «La tâche qui [lui] est dévolue le conduit très souvent à prendre en considération certains intérêts ignorés des autres droits et à adapter les concepts juridiques qu'il utilise en fonction des impératifs de la répression » (Danti-Juan, 1996, citation p. 482). À titre d'illustration, il est admis que le bon fonctionnement de l'ordre juridique impose que le sujet de droit soit identifiable afin que lui soient imputés individuellement ses droits et obligations. Aussi le Code civil organiset-il l'état civil. «Différente est la position du droit pénal : il admet les plaintes contre inconnu, la poursuite contre X. Il a même été jugé qu'une condamnation correctionnelle pouvait être prononcée contre un individu non identifié, quoique présent, et exécutée par lui. C'est que cette non-identification n'empêche pas qu'il y ait toujours un homme, un corps vivant, et cette réalité physique suffit au droit pénal » (Carbonnier, 2001, p. 235). En troisième lieu, le titre du nouveau livre du Code pénal, Des autres crimes et délits, est peu évocateur et traduit plus un malaise qu'une décision claire. Les embryons humains ont d'ailleurs rejoint les animaux dans ce dernier livre. Quant au Code civil, Jean-Pierre Marguénaud reconnaît lui-même que si le législateur a tenté maladroitement de distinguer les animaux des biens inertes et insensibles, il n'a pas extrait les animaux de la catégorie des choses appropriables. Enfin, l'article 544 du Code civil n'affirme le droit de jouir et de disposer des choses dont on est propriétaire que « pourvu qu'on n'en fasse pas un usage prohibé par les lois ou par les règlements », ce qui couvre aujourd'hui maintes règles restrictives.

Des auteurs ont pourtant rejoint Jean-Pierre Marguénaud. L'argument, semble-t-il déterminant pour eux, réside dans l'attribution à des associations de défense des animaux du droit d'agir en justice en vue de faire appliquer certaines règles pénales protectrices des animaux $^{12}$. Jean-Pierre Marguénaud articule cet élément avec les précédents : l'animal posséderait un « intérêt propre » à ne pas souffrir, « juridiquement reconnu » par le droit, et d'un représentant (en la personne de son maître ou d'une association de défense des animaux) susceptible d'agir pour la défense de cet intérêt. Dès lors, les conditions de la réalité technique de la personne morale, mises en évidence par Léon Michoud (1906) ${ }^{13}$, seraient réunies. Une « personne animale » devrait donc être reconnue sur le modèle de la personne morale, bien qu'avec des spécificités. L'auteur refuse en effet toute obligation passive ou action en responsabilité (Marguénaud, 1992, p. 382 ; Marguénaud, 1998, p. 207) à l'encontre de cette «personne » qui n'aurait d'ailleurs pas nécessairement de patrimoine (Marguénaud, 1992, pp. 388 sq.). Les animaux personnifiés relèveraient de la catégorie des animaux domestiques et seraient titulaires d'un nombre limité de droits : le « droit à ne pas souffrir inutilement» (Marguénaud, 1992, p. 422) pour tous les animaux domestiques, et le «droit à une durée de vie conforme à leur longévité naturelle » pour les « animaux artistes» et les «animaux que l'homme a choisi pour compagnon» (Marguénaud, 1992, p. 424). La personnalité juridique de l'animal pourrait également conférer des droits patrimoniaux de type créance alimentaire ou bénéfice de libéralités (Marguénaud, 1992, p. 410).

\footnotetext{
$\overline{{ }^{12} \text { Art. } 2-13}$ du Code de procédure pénale. Hermitte, in Burgat, janv. 2004, p. 50.

${ }^{13}$ On soulignera cependant que selon cet auteur ( ${ }^{\circ} 48$ bis p. 109) l'intérêt, support de la personnalité morale, est non seulement toujours un intérêt humain, mais surtout l'intérêt collectif d'un groupe d'hommes. L'intérêt défendu par les associations ou fondations est considéré comme un intérêt collectif humain et non pas comme l'intérêt de la cause défendue. Quant aux lois de protection des animaux, L. Michoud considère que l'intérêt que les animaux en retirent est un « effet réflexe» du droit objectif et non un droit propre.
} 
En l'état du droit, ces propositions d'extraction des animaux de la catégorie des choses restent doctrinales. Quelques décisions de justice, il est vrai, ont pu renforcer les auteurs dans leurs convictions. Ainsi, on a pu se référer à un jugement du Tribunal correctionnel de Strasbourg du 19 mai 1982 dont la motivation affirmerait que « tout animal est un être sensible ce qui exclut absolument la notion de chose $»^{14}$. Dans un jugement rendu le 2 mai $1985^{15}$, le Tribunal d'instance de Paris a retenu une formule proche, en affirmant que l'article du Code civil relatif aux frais engendrés par la conservation de la chose était «à l'évidence inapplicable en l'espèce, un chien domestique n'étant en rien une chose ». Cependant ces jugements sont atypiques. Des auteurs ont également relevé l'existence de jugements statuant sur le sort de l'animal après divorce sur le fondement des règles relatives à la garde de l'enfant plutôt que sur celles du droit des biens ${ }^{16}$. Peu nombreuses, ces décisions ont vu leur solution fermement censurée par la Cour de cassation ${ }^{17}$. Récemment, un propriétaire a introduit une requête devant le juge administratif en son nom et au nom de son animal pour contester l'ordonnance de placement en fourrière délivrée après constat que le chien de race Rottweiller circulait sur la voie publique sans laisse ni muselière et que le maître refusait de présenter aux forces de police le récépissé de déclaration exigé. Le juge administratif a écarté la requête présentée au nom de l'animal en relevant que « seules les personnes physiques ou morales peuvent ester en justice ». La demande, « en tant qu'elle est présentée au nom et pour le compte du chien Kaya », était donc « entachée d'irrecevabilité » ${ }^{18}$.

$\mathrm{Si}$ les propositions relatées trouvent peu d'écho en jurisprudence, elles exercent un réel attrait sur les personnes préoccupées du sort des animaux. Malgré les faiblesses de l'argumentation censée les justifier, les évolutions prônées leur paraissent améliorer la protection des animaux. Il est pourtant nécessaire de souligner que de telles propositions, spécialement celles visant à faire des animaux des personnes juridiques, entraîneraient de graves perturbations pour notre ordre juridique sans garantir une protection accrue des animaux.

\section{Inopportunité des propositions de personnification des animaux}

La personnalité juridique a été élaborée, comme l'origine théâtrale du mot « personne » l'indique ${ }^{19}$, pour permettre à l'homme d'apparaître sur la scène juridique et d'y jouer son (ses) rôle(s). C'est souvent oublié. Les tenants d'une personnification juridique de l'animal voient en elle d'abord un instrument de protection. Ils s'arrêtent à l'idée que la personnalité juridique confère un statut de sujet, à l'opposé du statut d'objet

\footnotetext{
${ }^{14}$ Trib. Correc. Strasbourg, 19 mai 1982, inédit, infirmé par CA Colmar, 12 janvier 1983, inédit, décisions citées par Levy-Bruhl, 1992, p. 96.

${ }^{15}$ TI Paris (16 $) 2$ mai 1985, Gaz. Pal. 1985 (2e sem.) somm., p. 234.

${ }^{16}$ TGI Lyon, 26 fév. 1973, inédit, cité, in : A. Couret note sous Civ. $1^{\text {re }}, 8$ oct. 1980, D. 1981, p. 361 ; TGI Évreux, ord. JAM, 27 juin 1978, Gaz. Pal. 1978, 2, p. 382. A rapprocher TGI Créteil, ord. JAM, 22 juin 1979, in A. Dosner-Dolivet et A. Scemama note sous Paris, 11 janv. 1983, Gaz. Pal., 1983, 2, p. 412. Cf. également CA Rouen, 22 nov. 1978, D. 1980, I.R., p. 75, obs. A. Bénabent. Pour une analyse, F. Pasqualini, 1997, Chron., p. 257.

${ }^{17}$ Civ. 1re, 8 octobre 1980, D. 1981, p. 361, note A. Couret.

${ }^{18}$ TA Strasbourg, ord. réf., 23 mars 2002, n 0201013, P. Welsch c/ Préfet de la Moselle, Dictionnaire Permanent Bioéthique et Biotechnologies, Bulletin 114, 26 avril 2002, n 9.

19 «Personne » vient du latin persona qui désigne d'abord un masque de théâtre, puis le rôle attribué à un masque, c'est-à-dire à un type de personnage ( $v^{\circ}$ Personne, Dictionnaire historique de la langue française, A. Rey (dir.), Le Robert, 2000).
} 
assimilé hâtivement au régime de libre exploitation ou de libre disposition. Pourtant, intégrer purement et simplement les animaux dans la catégorie des personnes physiques ne paraît ni souhaitable ni véritablement possible. Quant à la proposition de personnalité animale de pure technique juridique, elle apparaît inopportune.

\section{L'impossible intégration des animaux dans la catégorie des personnes physiques}

Les partisans d'une personnification de l'animal présentent souvent le droit positif comme la résultante d'une vision cartésienne du monde subordonnant fallacieusement la qualité de sujets de droit à la rationalité de l'individu ${ }^{20}$. Persuadés que les résistances des milieux juridique et politique s'expliquent par une appréhension du monde scientifiquement dépassée qui n'a perduré qu'en raison de sa coïncidence avec une conception égoïste de l'humanisme, ils cherchent à démonter les rouages de l'argumentation adverse. Ils font alors remarquer que tous les hommes ne sont pas à même d'assumer un rôle actif ou de faire face à leurs obligations et que ce handicap conduit à désigner un représentant pour défendre leurs intérêts. Or la loi autorise désormais les associations de protection des animaux à exercer les mêmes droits que la partie civile pour les infractions pénales que sont les mauvais traitements, les actes de cruauté (ou sévices graves) et l'abandon. Selon les partisans d'une personnification animale, il s'agirait du même type de représentation.

En réplique à cet argumentaire, plusieurs niveaux de réponse peuvent être envisagés. Sur le terrain de la technique juridique, on fera valoir que la qualité pour agir, conférée de manière très limitée par la loi aux associations de protection animale, diffère nettement du mécanisme juridique de la représentation d'un enfant par ses parents ou d'un incapable par son tuteur.

Sur un registre plus fondamental, une première réponse consiste à dénoncer les risques inhérents à toute comparaison entre les animaux et les êtres humains privés de volonté.

Certains auteurs dénoncent même une forme d'anti-humanisme chez les militants de la cause animale. Tel est le sens du propos développé par Luc Ferry dans Le nouvel ordre écologique (Ferry, 1992). On peut certainement reprocher à l'auteur une regrettable tendance à amalgamer des courants ou doctrines diverses ${ }^{21}$, ainsi qu'une interprétation alarmiste des discours prônant une philosophie décentrée. Luc Ferry va jusqu'à évoquer le régime hitlérien à titre d'ordre juridique particulièrement protecteur pour les animaux (Ferry, 1992, pp. 181-209 ; Ferry et Germé, 1994, pp. 505 sq.). La démarche est contestable

\footnotetext{
${ }^{20}$ Pour une critique de cette assimilation entre conception cartésienne du monde (objet de maîtrise et de possession pour l'homme), anthropocentrisme et toute puissance du sujet de droit, voir Y. Thomas, « Le sujet de droit, la personne et la nature. Sur la critique contemporaine du sujet de droit », Le débat, n ${ }^{\circ} 100$, mai-août 1998, p. 85.

${ }^{21}$ L'auteur assimile en effet de manière critiquable les militants de la cause animale et ceux de la cause écologique. Certaines questions ne trouvent pas les mêmes réponses selon que l'on entend préserver le milieu naturel dans lequel l'homme évolue ou les animaux. Selon la théorie adoptée la démarche écologiste peut accepter, voire exiger une forme de régulation des espèces animales, y compris par la suppression de certains individus. Cette solution n'est évidemment pas compatible avec le respect de la vie et de la sensibilité de l'individu animal. Il faut également préciser que de nombreux courants s'opposent au sein du mouvement écologiste comme au sein des partisans de la cause des animaux.
} 
en ce qu'elle semble associer nazisme et protection ou personnification des animaux. Plusieurs éléments invalident cette assimilation. D'abord, la législation allemande de protection des animaux adoptée sous le III $^{\mathrm{e}}$ Reich est issue de propositions formulées avant l'émergence du nazisme ${ }^{22}$. Ensuite, ce n'était ni la première législation protectrice des animaux en Europe, ni le premier texte protecteur des bêtes en Allemagne ${ }^{23}$. La Grande-Bretagne faisait déjà figure de précurseur en la matière. Enfin, l'étude des textes révèle que la protection accordée par les lois allemandes de 1933 n'allait guère plus loin que les textes aujourd'hui applicables dans la plupart des pays européens, à l'exception de la suppression de principe de l'expérimentation animale. Cette dernière disposition est présentée comme le symbole de la déviance nazie qui protégeait les animaux pour mieux livrer des hommes aux recherches insupportables des savants du Reich. Toutefois, d'après les travaux d'Élisabeth Hardouin-Fugier, l'interdiction de principe s'accompagnait de multiples exceptions et la loi de 1933 n'avait pas abrogé la loi de 1931 imposant aux chercheurs de recourir aux animaux avant d'expérimenter sur des hommes (Hardouin-Fugier, 2002, p. 62). Quoi qu'il en soit, cette opposition caricaturale entre protection des hommes et protection des animaux ne saurait convaincre. Pour mépriser et massacrer les hommes, il n'est pas nécessaire d'aimer les animaux et de vouloir faire évoluer les lois en leur faveur. On notera au demeurant que de grands humanistes furent partisans d'une protection légale des animaux contre les mauvais traitements : Voltaire, Victor Hugo, Victor Schoelcher et Pierre Larousse par exemple. Pour finir, il faut souligner que les lois de 1933 maintinrent les animaux dans la catégorie des objets de droit et ne créèrent pas de personnalité juridique animale. De toute évidence, la référence au régime nazi n'est pas pertinente pour contester les mouvements de protection animale et leur revendication personnificatrice.

Éminemment contestable, l'analyse de Luc Ferry n'en révèle pas moins les crispations suscitées par la question de la personnification juridique des animaux. Ainsi, certains des arguments développés pour convaincre de la pertinence d'accorder des droits aux animaux peuvent être utilisés à d'autres fins que l'élargissement de la « communauté des égaux ». Si les personnes sensibles au sort des animaux ne sauraient pour cette seule raison être taxés d'anti-humanisme, leur argumentaire fait craindre une récupération au service de causes moins nobles. On pourrait en effet ne retenir que l'idée initiale d'une continuité biologique pour conclure que seuls les êtres effectivement raisonnables sont dignes d'être sujets de droit. Les propos des penseurs utilitaristes partisans de la libération animale nourrissent cette crainte. Exposant le «projet grands singes », Paola Cavalieri écrit qu' « on justifie d'ordinaire le traitement différencié des êtres humains et des non-humains par une correspondance entre le fait d'appartenir à l'espèce Homo sapiens et celui de posséder les caractéristiques moralement pertinentes. Inversement, être non humain, c'est être dépourvu de ces mêmes caractéristiques » (Cavalieri, 2000a, $\mathrm{n}^{\circ} 108$, p. 157 $)^{24}$. Cependant, il est devenu difficile, selon elle, de défendre cette distinction : d'une part, « nous savons aujourd'hui que nous partageons avec les autres animaux nombre de nos gènes et une histoire évolutive commune » (Cavalieri, 2000a, p. 158);

\footnotetext{
22 Sur ce point, Hardouin-Fugier, 2002, p. 53.

${ }^{23}$ Pour une présentation du droit allemand, mais aussi des autres droits européens, en matière de protection animale avant la seconde guerre mondiale, $c f$. P. Giberne, 1931, pp. 48-85.

${ }^{24} C f$., également, Cavalieri, 2000c, n 109, p. 158, et 2004, p. 75.
} 
d'autre part, « nous avons toujours su, dans notre espèce, la présence d'individus non paradigmatiques, qui sont irrévocablement dépourvus de caractéristiques jugées typiquement humaines : les handicapés mentaux, les demeurés et les séniles ». Or, « sitôt que nous renonçons aux différences de nature, l'appartenance à l'espèce devient une simple caractéristique biologique parmi d'autres et ne saurait plus justifier l'idée que, quelles que soient leurs qualités empiriques, les humains non paradigmatiques doivent bénéficier du même statut moral que les membres paradigmatiques de notre espèce » (Cavalieri, 2000a). Au contraire, les découvertes des sciences cognitives et de l'éthologie tendent à démontrer que, si l'on adopte une définition ouverte de la raison et de la conscience de soi, les grands singes pourraient être intégrés à la communauté humaine. Ainsi, « dès lors qu'ils satisfont aux critères humanistes du statut de première classe en tant que patients moraux, l'impératif de cohérence exige qu'ils bénéficient de la même protection éthique fondamentale que nous » (Cavalieri, 2000b, p. 162).

Le risque de dérapage est perceptible. En termes justes, Jean-Claude Guillebaud insiste sur le malaise ressenti à la lecture de cette démonstration. " Singer et Cavalieri », écrit-il, «estiment [...] que, si l'on accorde les droits liés au statut d'humain à ces personnes dont l'entendement est diminué, alors il faut accorder le même privilège à certains animaux. [...] Ils trouvent injuste que l'on fasse bénéficier ces handicapés d'un statut supérieur qui ne correspond pas à leurs capacités mentales réelles. Faisant cela, ils introduisent au cœur même du principe d'humanité une distinction, une hiérarchie [qui] débouchent de facto sur l'idée d'une sous humanité dont l'histoire garde un sinistre souvenir. [...] Sauf à se réfugier dans une fausse naïveté, on ne peut nier que le raisonnement est virtuellement - et abominablement - réversible » (Guillebaud, 2001, pp. 55-56). Élisabeth de Fontenay formule les mêmes inquiétudes : il est certainement possible de repenser de façon critique « la vieille échelle des êtres » sans pour cela " abaisser les uns pour élever les autres et surtout [...] utiliser comme repoussoirs ceux des êtres humains qui sont dépourvus des qualités prétendument caractéristiques de l'espèce humaine » (de Fontenay, 2000, p. 142). La philosophe tient ceux qui recourent à de tels arguments pour « dangereux ». En réponse, Paola Cavalieri affirme ne pas penser « qu'on puisse traiter comme il nous plaît des êtres qui ne sont pas des agents moraux : des enfants, par exemple, ou toute idée de potentialité mise à part, les malades mentaux ». S'exprimant également au nom de M. Peter Singer, elle écrit : « Nous pensons, au contraire, que leur statut moral est le même que celui des membres plus rationnels de notre espèce. Du fait, précisément, de leurs limites mentales, ils méritent davantage encore, et non moins, notre protection morale » (Cavalieri, 2000a, p. 185). Pourtant, la lecture d'un ouvrage récent de Peter Singer laisse subsister le trouble. Exposant ses idées sur l'utilisation d'animaux dans des expériences scientifiques, l'auteur écrit : « Des adultes normaux ont des capacités mentales qui les font souffrir davantage que des animaux dans les mêmes circonstances. Par exemple si nous décidions de procéder à des expériences scientifiques extrêmement douloureuses, voire mortelles, sur des adultes humains normaux, kidnappés au hasard dans un jardin public, les adultes pénétrant dans les parcs auraient peur d'être kidnappés. La terreur produite serait une forme de souffrance qui s'ajouterait à la douleur de l'expérimentation. Les mêmes expériences réalisées sur des animaux non humains causeraient une souffrance moindre, car les animaux n'auraient pas d'avance peur d'être kidnappés pour subir des expériences. [...] Notons que le même argument donne des raisons de préférer qu'on utilise, pour les expériences, des enfants humains, par exemple 
des orphelins, ou des personnes gravement handicapées mentales, car les enfants ou les handicapés mentaux n'auraient aucune idée de ce qui va leur arriver. De ce point de vue, les animaux non humains, les enfants et les personnes gravement handicapées mentales entrent dans la même catégorie ; et si nous usons de cet argument pour justifier que cette expérience soit réalisée sur des animaux non humains, il faudra se demander si nous sommes prêts à permettre des expériences sur des enfants humains et sur des adultes souffrant d'un grave handicap mental $\gg .^{25}$

Les partisans d'une évolution juridique du statut de l'animal qui s'appuient sur une telle démonstration ne perçoivent-ils pas que le raisonnement justifie autant une personnification de certains animaux qu'une réification de certains êtres humains? Une réflexion sur les risques inhérents à cette argumentation s'impose, quel que soit l'objectif visé par la personne qui la développe.

Une fois ces remarques formulées, il faut tenter de répondre sur le fond aux arguments avancés par les défenseurs de l'animal sujet de droits. Notre système juridique accorde effectivement des droits aux individus qui ne peuvent s'en prévaloir sans l'aide d'un représentant. Il est fréquent de justifier cette solution par l'idée que l'infans deviendra un jour un adulte autonome ou que l'aliéné est un malade que la médecine a vocation à soigner tandis que l'animal est frappé d'incapacité permanente. La réponse est pertinente, mais il reste des individus dont on pense qu'ils ne recouvriront jamais suffisamment d'autonomie pour être qualifiés d'individus libres et raisonnables. Il faut admettre, si l'on suit le raisonnement utilitariste, qu'en leur accordant la personnalité juridique sans condition, alors que nous la refusons aux animaux, nous faisons preuve de « spécisme ». Pourtant, ce choix est-il contestable ? La question peut sembler choquante de cynisme, mais comment nier que le droit est une création humaine qui n'a de sens que pour les humains (Amselek, 1997, p. 337) ? Dans une perspective philosophique, lorsque nous réfléchissons sur la nature humaine et sur la place de l'homme dans le monde, lorsque nous nous interrogeons sur les valeurs à promouvoir, l'animal peut devenir un «prochain » (Burgat, 1997) également respectable. Dans une perspective juridique, lorsqu'il s'agit d'organiser la vie des hommes en société, de déterminer leurs comportements, l'animal ne peut être qu'un élément extérieur, un objet du droit. Cela n'empêche pas d'incriminer les comportements attentatoires à la vie ou à la sensibilité animale. Les humains sans voix, auxquels le masque de la personnalité est attribué sans souci de réalisme, sont les enfants, les parents, les frères et les sœurs d'humains conscients, libres et raisonnables. Pour ces derniers ${ }^{26}$, parce que toute autre solution leur paraîtrait inique, le droit doit accorder le statut de personne aux « individus non paradigmatiques ».

\footnotetext{
${ }^{25}$ P. Singer, 1997, p. 67. Rappelons, du même auteur, La libération animale, 1975, p. 49 : « Les chimpanzés, les chiens, les porcs et les membres adultes de bien d'autres espèces dépassent de loin un enfant au cerveau endommagé, du point de vue de leur capacité qui pourrait raisonnablement être considérée comme donnant une valeur à la vie, car même avec les soins les plus intensifs, certains enfants gravement déficients ne pourront jamais atteindre le niveau d'intelligence d'un chien. » Dès lors invoquer l'appartenance de ces enfants à l'espèce humaine « comme base pour accorder un droit à la vie à l'enfant sans en accorder aux autres animaux, constitue du spécisme à l'état pur » (p. 52).

${ }^{26}$ C'est-à-dire pour la société dans son ensemble. Rapp. F. Terré et D. Fenouillet, Droit civil. Les personnes, la famille, les incapacités, préc., $\mathrm{n}^{\circ} 9$, p. 9: «Il y a en tout homme vivant en société l'être individuel et l'être social, pris en considération par le groupe et, à ce titre, traité comme un sujet de droit. »Cf. également, J. Carbonnier, Flexible droit, op. cit., pp. 232 sq.
} 
Lorsqu'un homme est dans l'incapacité totale d'assumer ses obligations ou d'exercer ses droits, un représentant prend en charge la défense de ses intérêts, non seulement parce que la loi l'y autorise - ou l'y contraint -, mais parce qu'il se projette dans cet humain vulnérable qui n'a pas eu ou n'a plus la possibilité matérielle d'agir. Or, si l'anthropomorphisme est un moyen de s'ouvrir au monde extérieur et à la douleur non humaine, il entraîne une distorsion de la réalité qui ne peut être méconnue ${ }^{27}$. La projection mentale d'un homme vis-à-vis d'un autre homme, si elle a ses limites, est au contraire un moyen fiable de conjecturer sur ce qui est souhaitable pour l'autre, du moins lorsqu'il s'agit de décisions importantes. Ce pouvoir, exercé pour et sur une autre personne, s'accompagne de contraintes réelles. Tous les incapables doivent être représentés. Les parents supportent la responsabilité des actes de leurs enfants ${ }^{28}$. Le représentant juridique d'un individu atteint de troubles physiques ou psychiques graves est, le plus souvent, celui qui le prend en charge. Si tous les animaux sensibles étaient des sujets de droit représentés par les associations de protection animale, ces dernières pourraient-elles assumer la responsabilité juridique du fait de ces animaux personnifiés ? À l'heure actuelle, le droit leur confie la possibilité de déclencher des poursuites et de demander dédommagement lorsque certaines infractions sont commises - possibilité également attribuée par le Code de procédure pénale à d'autres associations pour d'autres infractions -, mais il ne s'agit pas véritablement de représentation.

Si l'on écarte l'idée d'une intégration des animaux dans la catégorie des personnes physiques, on peut encore envisager de leur conférer une personnalité de «pure technique juridique », en quelque sorte « taillée » pour eux. Il faut alors s'interroger sur la portée et l'utilité de la proposition ainsi formulée.

\section{La discutable utilité d'une personnalité animale de pure technique juridique}

Jean-Pierre Marguénaud entend se détacher des « propositions anthropomorphiques » attribuant aux animaux les droits des personnes physiques en les revêtant d'un « vêtement trop somptueux » (Marguénaud, 1992, p. 379). La bonne attitude consisterait, selon lui, à reconnaître que l'animal est un "infans perpétuel » ou un "aliéné incurable » (Marguénaud, 1998, p. 207) au regard des exigences juridiques. Il faudrait alors admettre l'existence d'une personnalité animale de pure technique juridique, sur le modèle de la personnalité des personnes morales (Marguénaud, 1992, pp. 396-406 ; 1998, p. 210). Les droits conférés aux animaux se limiteraient pour l'essentiel à une protection corporelle ${ }^{29}$, plus ou moins étendue selon les « sujets » animaux envisagés. Jean-Pierre Marguénaud considère que les dangers inhérents à l'intégration des animaux au sein de la catégorie des personnes physiques seraient ainsi évités, la réflexion menée dans le champ de la technique juridique pure avançant loin des abîmes que la dimension symbolique du droit ne manque pas d'ouvrir.

\footnotetext{
${ }^{27}$ « Un lion pourrait parler, nous ne pourrions le comprendre » constate Ludwig Wittgenstein (1961, p. 356), lorsqu'il s'interroge sur notre capacité à deviner la pensée des autres.

${ }^{28}$ Responsabilité civile, aux termes de l'article 1384, alinéa 4, du Code civil.

${ }^{29}$ L'auteur considère cependant que l'on peut reconnaître des droits patrimoniaux à certains animaux, notamment en leur conférant une créance d'aliments à l'égard de leur maître ou la capacité de recevoir des libéralités (Marguénaud, 1992, pp. 409-410).
} 
La voie de la technique juridique pure est attirante. Il est pourtant difficile de savoir si cette éviction du symbolique convaincra hors de la sphère des spécialistes du droit. Jean-Pierre Marguénaud regrette en effet que le «débat relatif à la personnalité juridique des animaux [soit] considérablement faussé par la confusion de ces deux aspects largement contradictoires : quand les uns se bornent, plus ou moins consciemment, à réclamer la commodité d'une technique juridique, les autres réagissent, plus ou moins sincèrement, en termes d'humiliation anthropomorphique et d'anéantissement des droits de l'homme » (Marguénaud, 1992, p. 206).

Il est vrai que les termes du débat relatif au statut des animaux sont souvent outrés. Les arguments des protagonistes sont parfois déformés. Chacun est figé par le regard de l'autre dans une posture caricaturale. Pour regrettable qu'il soit, ce constat nous apprend à relativiser la portée des subtilités que nous pensions décisives. Le droit n'a en effet pas vocation à rester objet d'étude d'experts en analyse de texte. Une fois tissée par la navette législative, la norme s'offre aux regards et aux interprétations. Elle échappe à son créateur et les points de couleur disparaissent dans le dessin d'ensemble. Or, cette personnalité animale demeure une fiction conférant à l'animal le statut de personne. Le paravent de la technique juridique parviendra-t-il à occulter la dimension symbolique et anthropologique de cette catégorie juridique ? «Le savoir juridique a son langage qu'aucun pouvoir ne peut maîtriser. Ce langage porte les idées et propositions, non pas dans un sens indéterminé ou opposé à celui auquel songeait leur auteur, mais dans une direction quelque peu différente. Des sens enfouis ressurgissent après quelques années et l'emportent sur ceux qui avaient été recherchés, retenus et mis en avant » (Atias, 2002, p. 89). Or, force est de constater que le terme de «personne » est de ceux qui véhiculent une cohorte de sens et de théories avec eux. Au-delà des mots et des interprétations suscitées, le destin d'une création juridique n'est jamais tracé. Une fois élaboré, l'outil juridique peut être utilisé à d'autres fins que celles pour lesquelles il avait été forgé. Cela est encore plus net lorsque cet artefact a été élaboré à partir d'un modèle dont il subit, par contrecoup, les transformations. Ainsi, la personnalité juridique des personnes morales - servant de modèle pour la personnalité animale - a progressivement été gagnée par le développement des droits extra-patrimoniaux, qui lui étaient pourtant initialement étrangers $^{30}$. Rien ne permet donc de penser que les droits attachés à la personnalité animale qui nous est proposée aujourd'hui seront les mêmes demain. Une fois mis à la disposition des plaideurs, l'instrument technique peut être sollicité de manière inattendue et se transformer en conséquence.

D'ailleurs, une telle réduction de la personne à sa dimension technique ne peut être reçue qu'à la condition de mesurer l'étendue du changement conceptuel réalisé. En tant

\footnotetext{
${ }^{30}$ Pour une application de l'article 9 du Code civil relatif à la protection de la vie privée à une personne morale : CA Aix-en-Provence, 10 mai 2001, D. 2002, somm., p. 2299, obs. A. Lepage. Voir également, CEDH 16 avril 2002, Sté Colas Est c/France (D. 2003, somm., p. 527, obs. C. Birsan ; D. 2003, juris. p. 1541, obs. A. Lepage ; JCP 2002, I, 153, n 11, obs. R. Besrour; JCP E 2003, I, 492, n 5, obs. J. Raynaud ; Europe 2002, n 307 , obs. N. Deffains) pour une application de l'article 8 de la Convention européenne de sauvegarde des droits de l'homme à une société commerciale. Les juridictions pénales admettent également la possibilité de sanctionner l'intrusion dans des locaux industriels, commerciaux ou professionnels sur le fondement de l'article 226-4 prohibant la violation du « domicile d'autrui » : cf. not. Crim. 7 février 1994, Droit pénal 1994, p. 129 ; CA Douai, 5 novembre 1993, Gaz. Pal. 1995, 1, somm. p. 178 ; CA Caen, 17 décembre 1997, Droit pénal 1998, comm. $\mathrm{n}^{\circ} 82$, obs. M. Véron.
} 
qu'instrument, la qualification de personne permet d'imputer des droits et des obligations. Il est de l'essence même du sujet de répondre de la dette qui lui est échue. En droit, il n'existe pas de personnes qui ne répondent jamais de leurs actes et de leurs engagements. L'homme trop atteint physiquement pour gérer son patrimoine ou agir en justice n'en continuera pas moins d'être tenu des dettes contractées en son nom, lesquelles seront imputées sur son patrimoine. L'homme atteint d'un trouble mental répond civilement de ses fautes ${ }^{31}$, et peut même en répondre pénalement si le trouble n'a pas totalement occulté sa conscience ${ }^{32}$. En tout état de cause, sa responsabilité ou son irresponsabilité pénale ne sera admise qu'au cas par cas, après démonstration de la privation de discernement. Seul l'enfant en très bas âge peut être considéré comme présumé irresponsable pénalement ${ }^{33}$, mais il est désormais civilement responsable de ses actes ${ }^{34}$. Les personnes morales peuvent être responsables pénalement ${ }^{35}$. Sur un plan civil, elles sont évidemment tenues de faire face à leurs obligations. Si l'on consacrait l'animal comme sujet de droits dans les termes suggérés par Jean-Pierre Marguénaud, il ne faudrait donc pas uniquement délester la personne de sa dimension symbolique, décision difficile - peut-être impossible - et qui ne serait pas sans conséquences ; il faudrait également admettre que le sujet de droit ne soit plus défini par sa capacité à être titulaire de droits et d'obligations. Le changement serait radical. Il est ardu de mesurer le bouleversement ainsi opéré. En effet, il n'est pas sûr que la distinction entre les personnes et les choses garde sa pertinence si la personnalité ne remplit plus la fonction traditionnelle d'imputation d'obligations.

D'ailleurs, cette réforme est-elle justifiée par le projet de personnalité de «pure technique » juridique ? Sur le terrain de la fiction technique, il n'est pas inimaginable que les animaux sujets de droit soient aussi débiteurs d'obligations. Dès lors que l'on admet qu'ils puissent être titulaires de droits patrimoniaux et, notamment, bénéficiaires de libéralités (ce que propose Jean-Pierre Marguénaud), on ne voit pas, par exemple, ce qui s'opposerait à une action judiciaire civile de la victime contre l'animal qui lui a causé un dommage. Quant à la responsabilité pénale des animaux, elle semble envisageable sur le modèle de la responsabilité pénale des personnes morales. En théorie, rien n'empêche le législateur d'organiser une responsabilité pénale des bêtes. En termes purement techniques, les animaux pourraient devenir des sujets de droits et d'obligations. Si cette idée est rejetée par les partisans de la « personne animale ${ }^{36}$, c'est un signe que la discussion n'a pas été expurgée de considérations symboliques ou extra techniques.

Cette impression est renforcée par la constatation de divergences entre les idées défendues par Jean-Pierre Marguénaud et celles développées par Demogue au début du siècle précédent, bien que le premier considère la lecture du second comme fondamentale pour comprendre ses propres écrits (Marguénaud, 1992, p. 363 et pp. 386 sq.). Demogue affirme

\footnotetext{
${ }^{31}$ Art. 489-2 du Code civil.

${ }^{32}$ Art. 122-1 du Code pénal.

${ }^{33}$ Art. 122-8, alinéa $1^{\text {er }}$, du Code pénal et Ordonnance n 45-174 du 2 février 1945.

${ }^{34}$ Cass. Ass. plén., 9 mai 1984, arrêts Lemaire et Derguini (Bull. civ. $\mathrm{n}^{\circ} 2$ et 3), commentés notamment in D. 1984, p. 525, concl. J. Cabannes, note Fr. Chabas ; JCP 1984, II, 20256, note P. Jourdain ; RTD civ. 1984, p. 508, obs. J. Huet ; Defrénois 1985, p. 557 ; Civ. 2e 12 déc. 1984, RTD civ. 1986, p. 120, obs. J. Huet ; Civ. 2e 28 fév. 1996, D. 1996, p. 602, note Fr. Duquesne, D. 1997, somm., p 28, obs. D. Mazeaud, JCP 1996, I, $3985, \mathrm{n}^{\circ} 14$, obs. G. Viney.

${ }^{35}$ Art. 121-2, alinéa $1^{\mathrm{er}}$, du Code pénal.

${ }^{36}$ Cf. Daigueperse, 1981 ; Marguénaud, 1992, p. 382, et Marguénaud, 1998, p. 207.
} 
que « la qualité de sujet de droit appartient aux intérêts que les hommes vivant en société reconnaissent suffisamment importants pour les protéger par le procédé technique de la personnalité. Le substratum essentiel est ici l'intérêt protégé. » (Demogue, 1909, p. 630). Nous invitant à tirer «quelques conséquences pratiques des idées techniques » émises, l'éminent juriste propose de considérer comme des sujets de droit les animaux, mais aussi les morts, les générations futures et même les immeubles. Jean-Pierre Marguénaud, à l'inverse, retient que «l'animal, à la différence des monuments historiques et des immeubles classés, n'est plus protégé au nom de l'intérêt général, mais d'abord pour lui-même », ce qui justifie à ses yeux que seuls les animaux soient qualifiables de sujets de droit. Il est bien difficile cependant de ne pas songer que ce qui fait la différence entre les immeubles classés et les animaux ressort davantage de la rhétorique des valeurs que de la pure technique juridique.

Ces questionnements sur la possibilité d'extraire le symbolique de la discussion juridique, surtout lorsque la discussion porte sur un concept aussi fondamental que celui de personne, nous conduisent à poser la question de l'utilité de personnifier l'animal ${ }^{37}$. L'utilité et l'efficacité d'une telle fiction sont présentées comme les véritables justifications de la proposition ${ }^{38}$. Pourtant, l'incertitude sur les retombées psychosociologiques d'une telle entreprise conduit à formuler quelques doutes. Il existe un risque réel de réaction négative, certaines personnes se détournant alors du souci de protection animale. Nombreux sont en effet ceux qui s'inquiètent du discours sur la personnification des animaux. D'un point de vue d'efficacité de la lutte menée par les partisans de la cause animale, cette inquiétude peut être « contre-productive ». On voit donc ce que la théorie de la personnalité juridique animale peut enlever à la cause des animaux. Il faut s'interroger maintenant sur ce qu'elle pourrait lui apporter.

La personnalité est sollicitée en tant qu'instrument de protection. Dès lors, il convient d'étudier le champ d'application de cette protection d'une part, et le contenu des droits accordés d'autre part, pour vérifier l'opportunité du changement.

La question du champ d'application est déjà problématique. Jean-Pierre Marguénaud distingue ainsi selon que les animaux appartiennent à la catégorie des animaux sauvages ou domestiques, puis à celle des animaux de compagnie ou de rente. Les animaux sauvages demeurent des choses (Marguénaud, 1992, pp. 421-427), au contraire des animaux domestiques.

Pour ce qui concerne le contenu des droits accordés, Jean-Pierre Marguénaud distingue deux niveaux de personnalité juridique. Le critère de démarcation proposé se veut pragmatique. L'auteur estime qu' « on ne peut envisager que tous les animaux personnifiés jouissent en même temps de la même condition juridique : ou bien alors l'humanité serait exposée à un péril d'étouffement voisin de celui qui semble inhérent à une personnification anthropomorphique, ou bien les animaux préposés aux caresses ne pourraient pas être mieux protégés juridiquement que les animaux voués aux couteaux et aux fourchettes ». En conséquence, il propose de distinguer les «personnes animales

\footnotetext{
${ }^{37}$ Cf. l'article d'Y. Thomas, 1998, p. 92 : « Passons sur le montage de la personnification, qui ne résout rien en lui-même, puisqu'il se réduit à une technique de la représentation : la nature serait-elle mieux protégée, personnifiée et représentée par des organes légalement institués, que laissée à son statut de chose, mais de chose protégée par un statut particulier, par hypothèse d'origine légale, lui aussi ?».

${ }^{38}$ Marguénaud, 1992, p. 362 : « Dans cette perspective, on peut, sans difficulté, attribuer cette qualité à l'animal si elle est techniquement commode et efficace».
} 
ayant un simple droit à ne pas souffrir inutilement» et les «personnes animales ayant un droit à une durée de vie conforme à leur longévité naturelle » (Marguénaud, 1992, pp. 421-427).

Il est vrai que les animaux de compagnie sont les êtres vivants non humains les mieux protégés en droit positif. Pour autant, la démarcation proposée étonne, puisqu'elle repose sur des critères utilitaires (à quoi sert l'animal ?) habituellement contestés par les protecteurs des animaux. N'y a-t-il pas quelque difficulté à concilier l'argumentaire développé pour justifier la fiction d'une personnification animale, qui insiste sur la sensibilité, avec l'idée d'une « grande personnalité animale » (Marguénaud, 1992, p. 431) pour les chiens et les chats (refusée aux animaux de rente sujets de seconde catégorie) et la qualification de choses pour les animaux sauvages ? Perturbante pour l'ordre juridique existant, cette proposition risque de ne satisfaire ni les protecteurs des animaux, ni leurs opposants.

Surtout, l'objectif fixé - une protection plus importante et plus efficace des animaux - n'est pas assuré. Il est donc indispensable de s'interroger : l'objectif de protection implique-t-il la consécration d'une fiction présentant l'animal comme un acteur de la vie juridique ? Ce n'est pas établi. Le respect des textes peut dépendre de la perception que le justiciable a de l'importance accordée aux valeurs protégées. Dans cette perspective, le changement de catégorie pourrait être bénéfique aux animaux mais en raison de sa dimension symbolique, laquelle pourrait également susciter des réactions négatives non souhaitées.

\section{Conclusion}

$\mathrm{Au}$ terme de cet examen critique concernant son statut, il apparaît que l'animal a besoin d'être protégé des comportements humains, mais qu'il n'a pas nécessairement besoin de droits subjectifs pour cela. Le droit objectif peut prendre en charge cette protection, dans la mesure où il assure le respect des valeurs humaines telles que la pitié et le refus de la violence. En droit civil, il est possible, et même souhaitable, de réorganiser le droit des choses, plus généralement dénommé droit des biens (Carbonnier, 2004a, p. 1591). Le droit de propriété tolère déjà de nombreuses limites justifiées par l'intérêt général. Par la reconnaissance de l'existence de biens spéciaux et la construction d'un régime spécial pour l'animal (c'est-à-dire une somme de règles spécifiques aux droits exercés sur les animaux), il deviendrait possible d'améliorer la protection des animaux sans révolutionner notre ordre juridique (Desmoulin, 2006a, pp. 37-75). 



\section{Chapitre 12 \\ Culture de l'élevage et barbarie des productions animales}

JOCELYNE PORCHER

La question des frontières entre l'être humain et les autres animaux se pose socialement et politiquement aujourd'hui avec acuité là où les relations entre humains et animaux sont les plus proches et les plus anciennes, à savoir dans la relation domesticatoire et plus particulièrement en élevage. C'est-à-dire là où nous sommes historiquement engagés envers les animaux. L'élevage implique entre êtres humains et animaux d'élevage un rapport de travail, un rapport de production qui conduit, à court ou moyen terme, à la mort des animaux. D'où il est déduit par certains que l'élevage, en soi, est un rapport d'exploitation abusivement légitimé par l'intérêt économico-alimentaire lequel, en nos temps biotechnologiques, pourrait d'ailleurs bientôt appartenir au passé. L'absence de frontière entre humains et animaux condamnerait la relation de travail avec les animaux et justifierait de revendiquer leur « libération ». Pour d'autres, ce rapport d'exploitation supposé et ce rapport mortel réel reposent sur la conviction, et y conduit, qu'entre nous et les animaux existerait un monumental fossé, une frontière absolue qui justifierait l'usage sans merci que les «productions animales » font des animaux.

La question des frontières entre « humanité » et « animalité » est aporétique. Elle occulte une autre question, beaucoup plus intéressante et moins rhétorique, qui est celle des relations entre humains et animaux, celle de la construction de la subjectivité humaine par la relation aux animaux (Porcher, 2007, pp. 352-362). Le travail en élevage témoigne que la qualité de la relation entre humains et animaux d'élevage permet de penser à la fois l'absence de frontières et la nécessité du lien, c'est-à-dire, de l'attachement et du détachement.

À l'exception de quelques chercheurs qui s'avisent de leur poser des questions judicieuses, les animaux d'élevage n'intéressent pas les éthologues (Despret et Porcher, 2007). Parce qu'ils s'inscrivent dans le monde du travail, les animaux d'élevage sont le domaine réservé des comportementalistes et des biologistes sans pour autant, paradoxalement, que soit prise en compte la spécificité de la construction de l'animal d'élevage par le travail. 
Pour des éleveurs, cette question des frontières est-elle pertinente ? Il existe des différences certes dont la première est que nous tuons le cochon et non l'inverse. Mais de ces différences pouvons-nous déduire une séparation radicale entre nous et les cochons ? Non. Le travail en élevage avec les animaux, notamment avec les mammifères, enseigne au contraire au quotidien combien nous sommes proches, combien nous sommes les mêmes d'un tout qui est le monde vivant et la nature dans son ensemble, ce qu'il est plus convenu aujourd'hui d'appeler la biodiversité, tout ce qui vit ensemble, inter-agit, communique, se co-transforme et est inter-dépendant. Ce qu'expriment deux éleveurs de porcs : «Je ne vois pas de différences. Il y a une différence évidemment, mais il n’y a pas de frontières. Il y a des passerelles entre le monde des animaux et le monde des hommes... il y a un respect des animaux. Je n'adapte pas la nature à l'homme, je considère que c'est à l'homme de vivre en harmonie avec la nature, et à partir de là, il n'y a pas de frontières nettes entre les animaux et les hommes, ce n'est pas possible »; "L'homme fait partie de l'écosystème des êtres vivants, on fait partie tous du même milieu écologique... La différence entre nous et les animaux, c'est que nous on les mange $»^{1}$.

Ce propos renvoie d'une certaine manière au point de vue matérialiste de d'Alembert, mais avec d'autres conséquences : «Tous les êtres circulent les uns dans les autres, par conséquent toutes les espèces, tout est en un flux perpétuel. Tout animal est plus ou moins homme ; tout minéral est plus ou moins plante ; toute plante est plus ou moins animal. Il n'y a rien de précis en nature [...] naître, vivre et passer, c'est changer de formes... Et qu'importe une forme ou une autre ? Chaque forme a le bonheur et le malheur qui lui est propre » (Diderot, 1994, pp. 899-900).

L'élevage rend l'éleveur philosophe. Je parle ici d'élevage, c'est-à-dire d'éleveurs et d'animaux d'élevage, ou encore, d'un travail porteur de sens partagé et dont les rationalités sont multiples: économique, mais également relationnelle et identitaire. Les « productions animales » qui fournissent l'essentiel du volume des produits animaux et impliquent la majorité des animaux d'élevage, sont d'une tout autre nature.

Partant de l'hypothèse que l'élevage, sur le fond, n'a rien à voir avec les « productions animales », j'essaierai de creuser leurs différences et de montrer, non pas que l'élevage renvoie à un paradis perdu qu'il s'agirait miraculeusement de faire renaître, mais que l'élevage appartient à notre culture et qu'il est une culture dont nous ne mesurons ni la valeur ni la fragilité. Ce en quoi l'élevage est une culture apparaît à l'analyse de ce qui n'en est pas, de ce qui est sans culture et sans histoire, c'est-à-dire de ce qui est barbare. De ce qui est supposé résulter d'une évolution de l'élevage quand ce n'est qu'un avatar de notre société industrielle : les « productions animales ». Par «productions animales », j'entends les productions industrielles (production porcine, cunicole et avicole) et les productions intensifiées (production laitière, production de veaux de boucherie, de taurillons...). À partir du milieu du XIX ${ }^{\mathrm{e}}$ siècle, avec la naissance de la zootechnie comme « science de l'exploitation des machines animales», l'industrie des «productions animales » a progressivement absorbé la majeure partie de l'élevage tout en présentant alternativement, tel Janus et en fonction des enjeux, notamment des enjeux de communication, la face historique « élevage/éleveur», en la prenant abusivement à son compte, ou la face «modernité » " productions animales/producteur ». L'animal d'élevage, qu'il

\footnotetext{
${ }^{1}$ Les citations sont extraites d'entretiens auprès d'éleveur(e)s et de salarié(e)s réalisés par moi-même, ou avec la collaboration de Vinciane Despret (Université de Liège), ou encore, avec celle de Catherine Pasquier (Inra).
} 
soit convoqué sur une face ou sur une autre, continue d'être nommé « animal d'élevage » et d'être un animal d'élevage bien qu'il soit conçu comme un produit, souche Isa brown en production avicole ou Naima en production porcine, et traité comme tel par l'organisation industrielle du travail.

Ainsi que l'écrit Michel Henry : «La barbarie n'est pas un commencement, elle est toujours seconde à un état de culture qui la précède nécessairement et c'est seulement par rapport à celui-ci qu'elle peut apparaître comme un appauvrissement et une dégénérescence » (Henry, 1987, p. 13). La barbarie, c'est la destruction de la culture. «Qu'est-ce donc que la culture ? Toute culture est une culture de la vie, au double sens où la vie constitue à la fois le sujet de cette culture et son objet. C'est une action que la vie exerce sur elle-même et par laquelle elle se transforme elle-même en tant qu'elle est elle-même ce qui transforme et ce qui est transformé » (Henry, 1987, p. 14) ${ }^{2}$.

C'est un désir d'accomplissement. À partir de l'exemple de la production porcine, et en m'appuyant sur l'expression des travailleurs dans cette production, donc partant du travail subjectivement vécu, je voudrais faire apparaître cet appauvrissement, qui pour les travailleurs constitue une souffrance, et donc la richesse initiale, la culture, à l'origine de nos liens aux animaux d'élevage ${ }^{3}$. Je commencerai par revenir sur le statut et la place des animaux d'élevage dans notre société avant de questionner les différences entre élevage et productions animales du point de vue du travail.

\section{La nature, la société et l'animal d'élevage}

Si les éthologues ne s'intéressent pas aux animaux d'élevage, nous ne pouvons que remarquer que les chercheurs en sciences sociales, majoritairement, ne s'y intéressent pas non plus. La raison essentielle tient sans doute de façon déterminante au durable statut d'objet de l'animal dans les sciences humaines, que l'on retrouve très profondément enraciné chez certains anthropologues. Pour ceux-ci, l'animal est un être sans conscience, immuablement déterminé par la nature et/ou par son patrimoine génétique. L'homme, grâce à la conscience et au langage, se produit (par le travail) en tant qu'homme en s'extrayant de la nature tandis que l'animal (tous les animaux) y demeure irréductiblement rivé. Puisque les animaux sont sans conscience, ils ne sont que produits de la nature (les animaux sauvages) ou produits du travail humain (les animaux domestiques). Les animaux d'élevage ne sont que des choses issues du rapport de production des hommes à la nature. L'étude des animaux relève alors des sciences de la nature comme en relèvent celles du charbon ou de la forêt: une matière que le travail de l'homme transforme et dont il use, légitimement, à son gré.

En dépit des nombreux débats théoriques en cours sur l'esprit des bêtes, ces représentations sont très tenaces et fondent la relation aux animaux construite par l'organisation du travail dans les productions animales. C'est sur la base de ces représentations qu'est produite la matière animale que nous consommons. C'est sur la base de ces représentations

\footnotetext{
${ }^{2}$ Les italiques sont dans le texte.

${ }^{3}$ J'utiliserai le terme « élevage » pour faire référence au travail historique avec les animaux et à des éleveurs autonomes et «productions animales » quand il s'agit précisément de celles-ci. J'utiliserai le terme «éleveur » pour désigner des travailleurs revendiquant de faire de l'élevage et celui de «producteur » pour les travailleurs affirmant leur adhésion au système de «productions animales ».
} 
que sont détruits pour raisons économico-sanitaires les vaches, les cochons, les volailles. Assainissement des marchés et restructurations des filières passent aujourd'hui par la case « destruction » et plus précisément même par la case « valorisation ». Car la matière animale est semblable à la matière végétale ; elle est compostable et combustible : elle est source d'énergie. Les ingénieurs ne manquent pas d'idées pour faire de tous les « déchets animaux » des combustibles à haute valeur écologique. Après le charbon et le pétrole, à l'instar du colza, voici le poulet/vache/cochon. La filière porcine française produit 26 millions de porcs, elle produit aussi 130000 tonnes de cadavres de porcs par an (Mouret et Porcher, 2007, pp. 245-252). Ainsi que l'écrivait Diderot, tout est matière organique, tout est énergie potentielle. Le problème est que ce tout n'est pas rien.

Nombre de nos contemporains mais également de nombreux scientifiques, juristes et philosophes perçoivent fort bien que quelque chose de fondamental se joue actuellement dans les «productions animales » mais ils sont majoritairement impuissants à penser ce quelque chose pour ce qu'il est, c'est-à-dire un appauvrissement, une paupérisation collective par disparition progressive du lien avec les animaux d'élevage. La pauvreté ne peut apparaître comme telle que par rapport à la richesse de ce qui précède ou de ce à quoi elle peut être opposée. Encore faut-il connaître cette richesse. Je ne fais pas référence à une antériorité temporelle, qui renverrait à une évolution linéaire du meilleur au pire, bien qu'il y ait évidemment un processus de destruction lié à la modernité, mais à une diversité dans le temps et dans l'espace des représentations de l'animal et de la relation aux animaux fondant les pratiques de travail avec eux. Ce n'est pas le fait qu'il y ait eu hier des bons ou des mauvais bergers qui nous intéresse, au contraire de Peter Sloterdijk (2003, p. 149), mais qu'il y ait aujourd'hui des systèmes d'élevage et des bergers. Ce qui est important, c'est de ne pas les laisser disparaître, eux et leurs animaux, parce que nous confondons élevage et productions animales. La réflexion philosophique, comme le souligne du reste Peter Sloderdijk lui-même en une sorte d'autocritique inaperçue, est singulièrement inadaptée aux questions que posent les relations entre êtres humains et animaux.

La plupart des analyses partent du postulat que les processus domesticatoires sont une exploitation des animaux, une «monstrueuse cohabitation $»^{4}$. L'origine est définie comme un désastre ; la suite ne peut donc être que calamiteuse, et il y aurait une fatalité originelle au traitement industriel des animaux, un tragique destin avec lequel il faudrait enfin rompre ; soit en « libérant » les animaux, soit en poussant à son extrême le processus industriel de production de viandes grâce aux biotechnologies (c'est-à-dire en se passant des animaux), soit, ainsi que les évolutions récentes en témoignent, en faisant les deux. Qu'on les libère ou qu'on s'en libère, le résultat serait le même. Nous serions condamnés à ne plus travailler, à ne plus vivre, avec les animaux. Que les processus de «domestication » des animaux, loin d'être une exploitation, puissent être au contraire pensés comme une émancipation n'a pour l'instant retenu l'attention ni des philosophes ni des anthropologues.

\footnotetext{
${ }^{4}$ «L'homme et les animaux domestiques -l'histoire de cette monstrueuse cohabitation n'a pas encore été présentée de manière adaptée, et surtout, les philosophes n'ont pas voulu voir, jusqu'à nos jours, ce qu'eux mêmes ont à chercher au sein de cette histoire » (Sloterdijk, 2000, p. 34).
} 
Le fait que la «domestication des plantes » et la «domestication des animaux » soient liées ${ }^{5}$, que ce même mot de « domestication » soit utilisé pour représenter un usage des plantes et une relation aux animaux, et plus largement tout usage d'une ressource naturelle culturellement appropriée («domestication » du feu ou de l'électricité), entretient cette vision linéaire et plombée de la relation de travail aux animaux. Il est donc nécessaire aujourd'hui de les dissocier. Non pas parce que la plante serait définitivement plus « autre » que l'animal, quel qu'il soit (corail, ver à soie, chat, cochon...), de notre point de vue subjectif, comme en témoigne Proust, mais parce que la relation que nous avons avec elle est très différente : " Il admirait, au fond de la gracieuse vallée, sur une tige élancée une digitale violette, habitante silencieuse et brillante de ce lieu avec quelques gueules-de-loup qui étaient à quatre ou cinq en famille. Et Jean pensait à ce que c'est qu'un endroit de la terre où l'on passe, mais qui reste là au pied de son rocher ou au bord de son torrent, qui n'a jamais rien vu du reste du monde. (...) Jean eut envie de l'emporter avec lui, dût-il la déraciner, qu'importe, et il aurait voulu aussi emporter ce val, le ravir à cet isolement absolu, éternel, qui lui donnait pour la première fois le sentiment de cette chose qui n'en était pas une autre, qui était hors de toutes les autres, et ne pourrait jamais s'en approcher, en connaître, et avec qui le silence seul partageait la solitude. Puis il n'osa plus. On craint de toucher à ce qui est à ce point soi-même » (Proust, 1971, p. 470).

C'est parce qu'animaux et plantes ont été mis au même niveau dans l'analyse des processus domesticatoires appréhendés comme «exploitation», (je ne reviens pas ici sur la logique archéologique et zoo-agronomique de cette association, je m'intéresse à ses effets), que l'on peut aujourd'hui traiter l'animal d'élevage comme une «matière première $»^{6}$ et en user comme on utilise la matière végétale. Puisque nous avons domestiqué le colza pour finalement en faire du carburant, pourquoi ne ferions-nous pas de même avec les cochons ou les volailles ? Cela fonctionne. À quelques détails près, on composte, on incinère les cochons comme les végétaux. Si les autobus roulent au colza, pourquoi ne rouleraient-ils pas aux « déchets animaux »?

Le fait que l'élevage ne soit pas entièrement dilué dans les productions animales, pas complètement encore du moins, permet de penser en partie autrement, de proposer d'autres hypothèses et des perspectives un peu plus réjouissantes.

Ce qui est dramatiquement occulté dans le contexte industriel des "productions animales », c'est le travail. Ce sont les rationalités du travail. Celui-ci, s'il donne comme l'écrivait Marx, une seconde nature aux humains, s'il les transforme, transforme également les animaux. Le travail donne à l'animal ce qu'il nous donne à nous-mêmes, c'està-dire une seconde nature, de nouvelles possibilités d'accomplissement, de relations, d'expression de conduites - ce qui implique un rapport subjectif au monde humain du travail - et non pas de comportements. Le travail permet le lien. À la différence des pieds de colza ou de vigne, les vaches, les truies, les chevaux, les éléphants, les chameaux, sont impliqués dans le travail et ils le savent. C'est pourquoi l'élevage ne relève pas de l'exploitation mais d'une relation de production qui engage l'affectivité dans le sens de ses deux dimensions du pouvoir et de l'amitié (Porcher et al., 2004, pp. 275-290),

\footnotetext{
${ }^{5} \mathrm{Cf}$., par exemple, Haudricourt, 1987, pp. 37-46.

${ }^{6}$ « Produit de base, non élaboré, résultant d'opérations d'extraction, de distillation, de récolte, etc. », Le Petit Robert.
} 
l'intelligence et la communication. Une relation de production qui doit être aujourd'hui collectivement négociée. Et, notons-le, dans cette démarche de négociation, la sociologie a un rôle crucial à jouer.

Éleveurs et animaux domestiques vivent et travaillent ensemble depuis des millénaires, peut-être tout simplement parce qu'il est beaucoup plus intéressant et joyeux de vivre ensemble que de vivre séparés. Car l'élevage est une passion de joie. Cette joie n'est d'ailleurs pas réservée aux seuls éleveurs. Elle est partagée par tous ceux qui vivent avec des animaux et qui sont de facto des éleveurs petit format. Ne peut-on considérer en effet que les animaux « de compagnie » ont un « emploi de service » attaché à leur présence, de même que les animaux vivant dans les zoos ou dans les parcs animaliers? Le statut d' « animal d'élevage », du fait des glissements de statuts entre "sauvage » et « domestique » et à l'intérieur même du groupe " domestique » est aujourd'hui très flou et nécessite d'être revisité.

Les animaux d'élevage, du moins ceux qui vivent dans des conditions permettant un rapport autonome à leur monde et un rapport compréhensif au nôtre, peuvent témoigner de toutes sortes d'états et de sentiments que nous percevons aisément. Et la joie est l'un des sentiments les plus forts que nous puissions partager avec eux. Que les animaux voient finir cette relation à l'abattoir n'ôte rien à la nature relationnelle du travail. Ce que cette fin à l'abattoir questionne par contre, c'est la place de la mort dans le travail et les conditions d'une possible évolution de ce rapport mortel.

Il faut insister sur la profonde différence que fait la joie dans le travail des uns (les éleveurs) et des autres (les producteurs). La joie n'est ni la satisfaction ni le plaisir. La joie est une exaltation du sentiment d'exister, en relation. La joie et la tristesse, écrit Spinoza, renvoient à la connaissance du bon et du mauvais : «La connaissance du bon et du mauvais n'est rien d'autre qu'un sentiment de joie ou de tristesse, en tant que nous en sommes conscients » (Spinoza, 1954, p. 77). Quand un éleveur, évoquant le retour du troupeau pour la tétée des veaux, dit: «Moi quand j'ai le troupeau derrière qui marche, quand je les sens derrière moi, je suis le plus heureux des hommes », cet éleveur sait que le sentiment fugitif de joie qui l'assaille, et dont il est éminemment conscient, par son intelligence et par tous ses sens, la force de vivre et d'agir sur le monde qui court de ses pieds à sa tête, sont fondés sur le rapport positif au monde et aux animaux qu'il perçoit en lui, sur une harmonie, sur un bien-être partagé. Cette joie est une joie du corps vivant. C'est pourquoi la joie des éleveurs, partagée par leurs animaux, n'est pas le plaisir des producteurs. Le plaisir lié à la performance technique que les producteurs expriment, la « passion » qu'ils affichent opportunément dans la communication de filière ne renvoient qu'à des passions tristes; toute joie est impossible en systèmes industriels, en dépit du désir de certains travailleurs, car le traitement brutal et irrespectueux infligé aux animaux ne leur convient pas, tout leur être animal s'oppose à la puissance humaine et lui résiste.

«On fait tout pour avoir beaucoup de porcelets et quand on voit que les truies elles s'allongent et elles en écrasent trois, du coup on a peur de se faire engueuler $»$.

L'animal d'élevage est à la fois partenaire et objet du travail, de plus en plus objet et de moins en moins partenaire en fonction des objectifs qui construisent le lien; parfois aussi de plus en plus ami et de moins en moins collègue, ainsi que cela se passe par exemple en Asie pour certains éléphants débardeurs de bois, privés de travail par la mécanisation, ou en France pour les ânes ou les chevaux. C'est pour garder une relation 
avec les éléphants ou avec les ânes et ne pas les voir disparaître du monde humain que les éleveurs leur cherchent aujourd'hui un travail (promener les touristes par exemple) et tentent de faire partager le lien. Précisément parce que le travail n'obéit pas exclusivement à la rationalité productive et qu'il a d'abord des significations relationnelles. Il est plus facile d'entretenir une amitié avec un éléphant si vous partagez avec lui un rapport de travail qui assure votre revenu et sa pitance, que s'il devient, faute de travail, votre animal de compagnie, car alors sa présence et votre travail, sont dissociés. Faire de l'élevage, travailler avec les animaux, peut donc être tout simplement décrit comme un très bon moyen pour vivre collectivement et durablement avec les animaux.

\section{Élevage et productions animales}

Dans le cadre d'enquêtes sur l'emploi en production porcine industrielle, j'ai récemment rencontré des femmes salariées qui ont quitté la production et qui ont choisi de faire un autre métier (Porcher, à paraître). Elles ont quitté ce métier parce qu'il les privait de ce pour quoi elles l'avaient choisi, c'est-à-dire faire de l'élevage : être en relation, soigner, veiller au bien-être des animaux, réduire la souffrance, éduquer, être utile, éprouver et donner de la joie, s'accomplir. Elles ont quitté la production porcine pour d'autres métiers du soin et de l'éducation (aide sociale, aide-soignante, ambulancière, monitrice d'équitation) à la hauteur non pas de ce qu'elles quittaient mais de ce pourquoi elles le quittaient. Je cite leurs propos :

«L'important, c'est que je sais que je peux être utile aux autres. En porcherie, on est utile à qui ?»

«Puisque je ne peux pas soigner les animaux, je vais essayer de soigner les humains. »

«Personne ne disait qu'on faisait souffrir les animaux. »

«Dans ma relation aux animaux, j'avais peur de devenir insensible».

Ces citations concentrent les éléments les plus importants des rationalités du travail et des enjeux de reconnaissance qu'il porte. Ces femmes ont quitté la production porcine industrielle parce qu'elles voulaient faire de l'élevage mais qu'elles ne le pouvaient pas ; parce qu'elles ont dû faire face à une pauvreté du travail avec les animaux d'autant plus difficile à assumer qu'elle est ignorée. Le rapport industriel aux animaux est en effet présenté aux élèves, stagiaires ou salariés potentiels comme relevant de l'élevage. Il acquiert de ce fait une sorte de normalité historique, d'autant plus forte que toute autre forme de travail avec les animaux est discréditée par les filières de productions animales comme relevant du bricolage.

Le rapport spontané de ces femmes aux animaux, qui est un rapport d'éleveur, un rapport de soin et d'éducation réciproque, est étouffé par la rationalité industrielle : ne pas faire de sentiment, ne pas être sensible, ne pas se ridiculiser à parler aux animaux... Il faut énormément de temps alors au travailleur pour appréhender l'absence d'élevage comme un manque, et la durabilité de la relation d'élevage aux animaux dans le système industriel comme une perversion et un avilissement de soi-même et du rapport à la vie. Il n'y a pas d'élevage industriel, ces deux mots sont complètement antinomiques. Du fait de l'intersubjectivité des relations entre humains et animaux existe une inévitable permanence du lien même en systèmes industriels, parce que ce lien est désiré par les travailleurs et par les animaux, c'est lui qui donne le sens profond des choses, mais il est perverti parce que l'organisation du travail nie ce lien en tant que lien. Elle en use dans 
le management parce que ce lien est rentable mais il est réduit par l'encadrement des éleveurs et des salariés à une interaction; il perd ainsi sa valeur intersubjective, et devient un acte volontaire. C'est pourquoi il peut être fait aux travailleurs la double injonction d'être « l'ami de leurs truies » et d'être capable de leur donner un coup de masse sur la tête si elles ne prennent pas à l'insémination ou si elles ont des problèmes hépatiques ou des problèmes d'aplomb.

Pour que le travailleur prenne conscience que quelque chose ne va pas, qu'il est pris dans un processus qui l'éloigne dramatiquement de ce qu'il espérait à l'origine du travail avec les animaux, il faut que les stratégies individuelles et collectives contre la souffrance faiblissent ${ }^{7}$. Pour tenir au travail en effet, nous construisons des défenses qui nous protègent contre la souffrance, mais ces défenses ont également pour effet de réduire la pensée et la possibilité d'agir librement. Comme l'écrivaient Marx et Proust, c'est la souffrance qui met la pensée au travail, et c'est la pensée qui fait agir et qui permet de transformer la souffrance en bonheur et en joie.

Reprenons les points que les femmes citées soulèvent en rapport avec ce qui est appauvri, ce qui est détruit par les "productions animales », et au contraire en rapport avec ce qui est présent en élevage et renvoie à la culture en tant que rapport à la vie, une vie qui se construit, s'éprouve et s'enrichit dans la relation aux animaux. Je précise que nous sommes ici à la fois dans le domaine des représentations, des valeurs morales et du travail réel, c'est-à-dire de ce qui est effectivement partagé entre les travailleurs et leurs animaux. Il ne s'agit pas de comparer ce qui existe aujourd'hui avec ce qui existait il y a soixante ans ou même cent cinquante ans, mais de comprendre que tout ce qui existe aujourd'hui met en lumière les composantes essentielles de quelque chose qui est encore actuellement, qui s'appelle l'élevage, et qui est en train de disparaître. Non par hasard ou par mégarde, car il y a derrière cette disparition un ensemble convergents d'intérêts extrêmement puissants. Il sera difficile d'éviter dans cette très courte réflexion des comparaisons dichotomiques et quelque peu manichéennes mais, avec l'appui de Michel Henry, j'assume le manichéisme de l'opposition «barbarie/culture » en tant qu'elle éclaire de manière non ambiguë les profondes oppositions entre « élevage » et « productions animales ».

\section{«Être utile aux autres : en porcherie, on est utile à qui ? 》}

Sur cette question de l'altruisme, je renvoie à ce que propose Michel Terestchenko (2005). À l'opposition entre altruisme et égoïsme, l'auteur invite à substituer ce qui oppose «absence à soi » et «présence à soi ». «Absence à soi d'une individualité défaillante, inconsistante, prête à succomber à toutes les formes de domination, d'asservissement et de passivité, mais non pas nécessairement dénuée de tout "sens moral"; présence à soi, au contraire, d'un être doté d'une puissante ossature intellectuelle, spirituelle, morale, comme on voudra, d'un équilibre intérieur - j'emprunte l'expression à Nadejda Mandelstam - qui le rend capable de résister à l'oppression, à l'injustice, aux aliénations de l'idéologie dominante, capable de voir l'inacceptable, de discerner le mal comme tel et d'agir en conséquence » (Terestchenko, 2005, p. 18).

L'une des caractéristiques des éleveurs est cette présence à soi et c'est grâce à elle sans doute que l'élevage n'a pas encore disparu. Être au service des animaux est une

\footnotetext{
${ }^{7}$ Sur les stratégies défensives dans le travail, lire Dejours, 1998.
} 
représentation du métier d'éleveur à laquelle adhèrent une majorité d'éleveurs (Porcher, 2001). Et cela en dépit des injonctions sans cesse réitérées de l'encadrement agricole à produire toujours plus sans «considérations » pour les animaux, et même, ainsi que le souligne une salariée en systèmes industriels, quel que soit le prix à payer pour les animaux :

«Je pense que du fait qu'on doit produire tant de cochons par mois et par semaine, c'est produire à tout prix et à n'importe quel prix. S'il faut perdre deux coches pour gagner dix cochons parce qu'elles auront eu le temps de les mettre bas, même si elles sont dans un état pitoyable, c'est ça qui pousse ».

La question de l'utilité renvoie à celle de la reconnaissance. Ainsi que le souligne Christophe Dejours, pour être positif pour l'identité, le travail doit être reconnu : il doit être bien fait (jugement de beauté reconnu par les pairs); il doit être utile (jugement d'utilité par les usagers).

La majorité des éleveurs perçoivent leur travail comme une mission dont la part la plus importante est de «nourrir les gens». Du fait des critiques sociales contre les systèmes industriels, cette mission perd de sa visibilité. Si nous consommons en France les 26 millions de porcs que les éleveurs ont produits, nous ne leur en sommes pas très reconnaissants. Les travailleurs sont accusés de polluer, de maltraiter les animaux et de produire un aliment douteux. La reconnaissance (jugement d'utilité) n'est donc pas au rendez-vous du côté des consommateurs.

Du côté des pairs (jugement de beauté), elle ne l'est pas non plus. Les éleveurs, pris dans un processus accéléré de concentration des structures, sont dans une concurrence acharnée. La reconnaissance interne passe par les « cochons d'or » et par l'encadrement, c'est-à-dire par des résultats quantitatifs qui sont toujours à reconduire. La reconnaissance, par essence, est alors toujours repoussée plus loin.

Mais la reconnaissance dans le travail en élevage ne provient pas seulement des humains. Elle est aussi fournie par les animaux. Pour la prendre en compte, il faut admettre qu'il existe une intersubjectivité des relations entre travailleurs et animaux, ce dont ne doute aucun travailleur, même en systèmes industriels.

J'appelle ce type de reconnaissance «jugement du lien ». C'est la reconnaissance perçue par le travailleur comme étant accordée par les animaux. J'ai montré par ailleurs qu'en élevage cette reconnaissance s'inscrit dans un rapport de don dans lequel l'affectivité et la communication sont centrales (Porcher, 2002, pp. 245-262). Pour de nombreux éleveurs, les animaux, d'une certaine manière, portent un jugement sur le travail. Parce qu'ils sont impliqués dans le travail, parce qu'ils y collaborent de manière intentionnelle, parce qu'ils prennent des initiatives, ils sont en mesure d'exprimer de différentes façons leur avis sur le travail ; majoritairement, non pas sur les résultats du travail mais sur ses moyens. Plus largement, on trouve chez certains éleveurs, le sentiment que les animaux portent un jugement moral sur la relation de travail (« qu'est-ce que les animaux vont penser de nous? »).

En systèmes industriels, pour les travailleurs, le jugement du lien est faible. Les animaux ne disent pas merci, voire sabotent le travail. Je cite deux salariées actuellement en poste :

« J'ai l'impression des fois qu'une truie, ce n'est pas très reconnaissant quand même. On les soigne tout ça. Je pense qu'elles doivent apprécier en maternité par exemple pour une fouille si un porcelet est coincé, elles doivent apprécier quand même, ça leur évite de pousser trop, 
de s'épuiser tout ça, mais je sais que j'ai vu des truies que j'ai aidées, qui avaient une patte coincée, quelque chose comme ça, elles s'en foutent hein, elles n'en ont rien à foutre de nous après. Une fois qu'elles sont reparties, c'est bon quoi. Est-ce qu'elles se rendent compte ? Je ne sais pas si elles se rendent compte qu'on est là aussi pour les soigner... je pense que si les animaux parlaient on se ferait engueuler tous les jours ».

«Il y en a qui vont écraser impunément leurs petits, comme ça, on se demande des fois si elles ne le font pas exprès ; j'ai trop de cochons, c'est bon, je vais en enlever un ou deux parce que j'en ai trop. On se demande des fois, ça me bouffe ça. Je suis en rage des fois, je n'arrive pas à m'y faire, c'est ça surtout, je n'arrive pas à m'y faire (...) des fois je me dis que je préférerais être au sevrage ou ailleurs. Je l'ai dit plusieurs fois à mes patrons ça me dérange beaucoup parce que ça me bouffe de voir ces pauvres petits qui se font écraser ».

$\mathrm{Ce}$ jugement du lien, cette reconnaissance du travail par les animaux, cette collaboration est au contraire très présente en élevage. Je cite un éleveur de vaches : «Les vaches, j'ai toujours pensé qu'elles nous faisaient la grâce de pouvoir vivre à côté d'elles, même si nous on se donne le droit de vie et de mort sur ces animaux, et que cette grâce, elle t'inonde, elle te fait du bien. C'est un herbivore déjà qui a du temps pour faire les choses. J'ai toujours pensé que c'est un animal supérieur. Ca te renvoie à tes futilités, tu te débats pour pas grand-chose, comme quand elles te font la grâce de prendre un veau qui n'est pas le leur, elles le font pour te faire plaisir ».

\section{«Puisque je ne peux pas soigner les animaux, je vais essayer de soigner les humains »}

Cette phrase abrupte qui résume l'impuissance au travail ressentie par cette salariée renvoie aux conditions de vie des animaux en systèmes industriels. En élevage, les animaux sont pour l'essentiel autonomes et n'ont donc pas besoin de soins constants. Ce n'est pas le cas en porcheries où les truies encagées ne peuvent survivre sans l'intervention humaine. Il faut noter que ce désir de soin, notamment de la part des femmes mais pas seulement, est contraire au désir de liberté imputé aux animaux. Pour que le soin existe, il doit être rendu nécessaire. Une truie élevée en plein air n'a pas besoin d'assistance à la mise bas. Elle met bas et élève seule ses petits, le travailleur n'intervenant qu'en cas de problèmes. Cette relation distanciée aux animaux en élevage, qui renvoie à ce qu'Erwin Straus (2000) appelle la «compréhension symbiotique », est très différente du rapport industriel invasif que connaissent les salariées. Chez les salariées qui ont quitté le métier, la recherche d'une relation plus respectueuse aux animaux, - et plus largement aux individus, animaux et humains -, qui tienne compte de leur propre désir d'autonomie et ne soit pas cause de souffrance, a visiblement été prépondérante.

Le désir de nombreuses salariées en systèmes industriels est de réparer les dégâts du système, ce qui est une tentative inéluctablement vouée à l'échec du fait des marges de manœuvre très faibles qu'ont les travailleurs à l'intérieur du système, et donc affectivement très coûteuse. Il s'agit d'aider l'animal à supporter sa condition, comme s'il était malade, où plutôt parce qu'il est effectivement malade et soutenu dans sa claustration grâce à une importante panoplie pharmaceutique. Faire des piqûres est une activité récurrente. Tuer les animaux - « pour leur éviter de souffrir »- également ; notamment les porcelets chétifs issus des truies hyperprolifiques. Celles-ci produisent - c'est le mot - 15 à 20 porcelets par portée, voire plus, et un certain nombre sont très petits. Les salariés les tuent ou tentent de les faire survivre. Je cite une salariée en poste : 
«Je ne les tue pas les porcelets, c'est très rare que j'en tue. Non, mes petits, je n'arrive pas à les tuer. Non, je les laisse ou alors s'ils sont petits, je les tue dès la naissance, mais j'aime pas. Je sais qu'il y en a..., moi je m'occupe des petits, des tout petits, je les aide au maximum, et c'est vrai qu'on arrive à les sevrer, et je sais qu'il y en a dans certains élevages qui ne font pas ça. Enfin, il y en a une partie qui meure mais il y en a une grande partie qui est sauvée. Mais j'aime pas les tuer ».

Dans ces systèmes, rappelons-le, il n'y a plus d'infirmerie. Soit l'animal malade guérit rapidement, soit il est tué sur place, soit il meurt « de lui-même ». Il faut donc prendre aussi dans son sens littéral le constat de cette salariée qu'elle ne peut plus soigner. Je cite une autre salariée qui a quitté le métier :

«Les charcutiers, en règle générale, c'était au vermifuge (surdosé) qu'on les piquait, les plus gros, et puis, les truies, c'était aussi une injection de vermifuge qu'on faisait pour les achever, et autrement elles meurent d'elles-mêmes, on va pas chercher plus loin, on les met dans un coin et on attend».

En élevage, la relation de travail avec les animaux engage l'éleveur dans un rapport de soin. La maladie témoigne d'un échec du travail et de la relation, et ne renvoie pas à la norme mais à l'exception :

«Je ne supporte pas encore la perte d'un animal. Un veau qui me meurt, je suis révolté, je ne peux pas supporter. Autant si c'est une vache qui est malade que finalement elle arrive doucement à décliner, autant finalement je souhaite presque sa mort quoi, parce que c'est comme quelqu'un que l'on aime beaucoup, mais autant perdre un animal qui est à venir quoi, ça je ne peux pas le supporter. C'est quelque chose qui malgré 20 ans d'élevage, je vais dire que j'ai perdu des veaux comme tout le monde, mais jamais je m'y suis fait, je crois que je ne m'y ferai jamais et je ne m'y ferai jamais, c'est une révolte permanente ».

\section{«Personne ne disait qu'on faisait souffrir les animaux »}

En ces temps où les financements pour le « bien-être animal » abondent autant que les colloques sur ce sujet, la souffrance des animaux en systèmes industriels atteint probablement des sommets jamais vus. Cette souffrance est niée par les filières industrielles. Elle est niée historiquement à l'appui de la science, et plus récemment à l'appui de la problématique du « bien-être animal » et des innovations afférentes. C'est une souffrance soft qui se passe le plus souvent de bâton et de hurlements. C'est une souffrance liée au caractère impitoyable du système. Pour les travailleurs, admettre cette souffrance des animaux est extrêmement difficile, parce qu'il faut s'opposer au credo de la filière et à la pression idéologique forte de celle-ci, ainsi qu'en témoigne ces préconisations de la Fédération nationale porcine (FNP) ${ }^{8}$.

\section{Vendre l'image de l'élevage porcin}

- Ouvrir les élevages, donner la possibilité au citoyen de connaître ce qu'est un élevage de porcs. Des actions importantes de communication ont déjà été engagées avec l'appui du Centre de documentation des métiers du porc (CDMP). Mais chaque éleveur de porc doit concevoir la communication vers l'extérieur de son exploitation et notamment son

\footnotetext{
${ }^{8}$ Extrait du compte rendu de l'Assemblée générale de la Fédération nationale des éleveurs de porcs de mars 2006.
} 
entourage immédiat, comme partie intégrante de son métier afin de créer les conditions d'une meilleure acceptabilité de son élevage et réduire les risques de conflits ${ }^{9}$.

- Faire comprendre que «développement» est compatible avec une meilleure maîtrise de l'environnement, une meilleure gestion des nuisances et du bien être animal. Les débats auxquels les éleveurs ont à faire face vis-à-vis des citoyens, caricaturent et mettent en cause le plus souvent les élevages sur caillebotis. Il est indispensable de démontrer que chaque mode d'élevage à sa raison d'être et qu'il est aberrant d'opposer les systèmes. Il convient de faire comprendre que des élevages de taille plus importante, organisés de façon plus rationnelle quel que soit leur mode, aboutissent, le plus souvent, à une meilleure maîtrise de l'environnement, une meilleure gestion des nuisances et du bien être animal. Ils favorisent également l'amélioration des conditions de confort des travailleurs.

L'objectif de la FNP est d'impliquer les travailleurs dans le système industriel de façon à ce qu'il leur soit impossible d'afficher, et même de penser, tout désaccord, y compris « dans son entourage immédiat».

Le travailleur qui doute doit également faire face aux défenses viriles des collègues. Et à la difficulté éventuelle de retrouver un emploi. Il faut faire confiance à ses sentiments, à ses propres valeurs, face à tout ce qui dans votre milieu de travail dit que vous avez tort, parce qu'il faut bien nourrir les gens, qu'il faut bien qu'on produise en France parce qu'en Espagne c'est pire, qu'on ne peut pas faire autrement, que ça s'améliore quand même, que ce ne sont que des animaux, qu'ils sont fait pour ça, qu'ils ne connaissent que ça, qu'ils ne souffrent pas tant que ça, et qu'il y a des gens qui meurent sous les ponts tout de même, qu'il ne faut exagérer, qu'il faut être réaliste... Et puis, argument récurrent chez de nombreux travailleurs en souffrance : « il ne faut pas rêver »; la souffrance est partout, elle constitue l'essence même du travail puisque travailler c'est souffrir. Ce n'est pas le bonheur qui est au rendez-vous, c'est la compétitivité. Rêver est interdit.

«À un moment donné on se dit, enfin des fois on se pose la question, pourquoi j'ai pu faire ça,
comment j'ai pu aller jusqu'à là ? Mais je crois que je n'étais pas prête à dire non, à arrêter ça
à ce moment-là. Quelque part vous êtes... vous n'avez pas... Je pense qu'il y a une histoire
de courage aussi, de prendre une décision, prendre une décision, c'est aussi entendre... j'avais
besoin aussi d'être encouragée quelque part, et personne ne parlait de ça, personne ne disait
"on fait souffrir les animaux"... Il m'a fallu beaucoup de temps pour pouvoir oser le dire parce
que c'est quelque chose de l'ordre de l'inentendable. Il ne faut pas oublier que l'on est dans
le milieu rural et s'il y a un endroit où culturellement on implique la souffrance dans la vie,
c'est bien en milieu rural et en milieu agricole, où on a l'impression que l'on ne peut gagner
les choses que par l'effort et la souffrance ». L'élevage, parce qu'il est un métier qui confronte à la vie et à la mort, est générateur de plaisir et de souffrance. Chez de nombreux éleveurs, la recherche du plaisir et de la joie pour eux-mêmes et pour leurs animaux, dans le mystère de la relation, est très forte :

«On fait un métier pourquoi pour gagner sa vie, mais aussi pour avoir des plaisirs. J'ai été dans des élevages industriels, j'ai des copains qui en font, je ne supporte pas, je m'énerve, je deviens..., il y a des odeurs, non je ne supporte pas. Vous savez, une vingtaine de truies en plein air, à côté des porcelets qui pètent la santé, ça peut être aussi passionnant. Les performances économiques mettent au second plan tout un tas d'autres aspects ».

\footnotetext{
${ }^{9}$ C'est moi qui souligne en mettant en italique cette recommandation.
} 
«Ca fait plaisir de voir les animaux venir vers nous, gambader dans la prairie. Ca fait plaisir quand vous êtes tout seul et que vous amenez un grand troupeau, elles sont une quarantaine de truies, vous êtes tout seul et elles vous suivent, je trouve ça fabuleux, surprenant ».

\section{«Dans ma relation aux animaux, j'avais peur de devenir insensible »}

Le travail nous change. Car, comme l'écrivait Marx, c'est le travail qui construit nos représentations et non l'inverse ${ }^{10}$. Ainsi que le précise Christophe Dejours, le travail est une occasion d'accroissement de notre sensibilité (Dejours, 1993). Il peut l'accroître et ainsi enrichir notre rapport au monde et aux autres, mais il peut aussi la détruire. Cette destruction de la sensibilité, ce travail de détachement, ce passage à la barbarie s'opère progressivement et en grande partie à l'insu des travailleurs grâce à l'habitude. Grâce à l'habitude, certaines pratiques ne font plus mal aux travailleurs bien qu'elles continuent de faire mal aux animaux. Mais les travailleurs ont appris qu'elles sont «nécessaires », comme est nécessaire l'ensemble du système de production; ils se sont « rentrés ça dans la tête ». Les défenses solides qu'ils ont construites pour ne pas souffrir les ont détachés d'une partie de leurs sentiments, de leurs affections et du sens qu'ils donnent aux choses. Elles les ont détachés également de tout moyen de penser l'élevage autrement et de le penser dans l'avenir (Porcher, 2006, pp. 78-89).

«La truie, elle est là pour donner des cochons. C'est sûr qu'une truie ne doit pas rester vide. On ne va pas avoir dix vides en gestantes. C'est fini pour elle, elle n'a pas le droit à l'erreur. Le problème, c'est qu'elle mange de l'aliment alors qu'elle ne produit pas. Nous, c'est tellement logique. On est tellement habitué. Une jeune qui revient une fois, ça va. Une vieille elle va à l'abattoir. Une chance, ça coûte trop cher, ça désorganise toutes les bandes. Pour nous c'est une routine. Elle est obligée de produire».

«Il ne faut pas être sensible, de toute façon si on est sensible, soit on arrête, soit on devient moins sensible».

«Les 3 ou 4 premiers mois (tuer les petits) je ne le faisais pas, je préférais les laisser claquer tout seul, mais bon des fois ils mettent deux jours, alors je me dis non, je vais abréger quand même les souffrances... alors je me suis décidée quand même à les tuer. Bon maintenant je ne pleure plus quand je le fais, je me suis habituée mais j'ai eu du mal».

Le travail industriel entraîne une déception de la relation aux animaux qui conduit certains salariés à quitter le métier, même si cette raison n'est pas nécessairement la première affichée. Car élever et produire, ce n'est pas la même chose et c'est ce dont ces travailleurs témoignent :

Contrairement à ce qui se passe en systèmes industriels, le travail en élevage, grâce à la relation avec les animaux, permet d'exprimer des potentialités affectives particulières. Il ne rend pas « insensible », il rend au contraire « sensible », c'est-à-dire. capable de percevoir, de sentir, de ressentir.

« J'ose exprimer mes sentiments. J'ose dire, moi j'aime mes animaux, oui c'est vrai les animaux font partie de ma vie, oui c'est vrai quelque part, c'est aussi ma famille».

Il accroît la sensibilité et permet une intelligence des choses accrue. Le «sixième sens » des éleveurs, l'intuition, est une compétence sensible construite par la relation aux animaux.

10 « Ce n'est pas la conscience qui détermine la vie, mais la vie qui détermine la conscience » (Marx et Engels, 1975, p. 51). 


\section{Conclusion}

Le passage de l'élevage à la production animale entraîne un appauvrissement de la relation entre l'homme et l'animal qui porte sur l'affectivité, la sensibilité, la communication. Or, pour Michel Henry, là précisément est la culture. L'affectivité est à l'origine de la connaissance. C'est par notre sensibilité que nous connaissons le monde et que nous appréhendons la vie. Quand les éleveurs, quand nos concitoyens affirment aimer leurs animaux, il faut prendre cette affection au sérieux et comprendre qu'elle n'existe que dans la réciprocité ${ }^{11}$. Que le travail en élevage ait des fonctions relationnelles et économiques n'ôte rien à sa richesse. Cette relation nous en apprend beaucoup sur ce qu'il en est de l'humain, de l'animal et de nos différences, et surtout de ce qui nous rassemble. Sans doute, contrairement au « canon de Morgan $»^{12}$, serait-il plus efficace de poser des hypothèses généreuses envers les animaux. Mais il est vrai que la générosité nous manque. Nous pouvons difficilement multiplier les charniers économico-sanitaires et reconnaître les animaux pour ce qu'ils sont et ce que nous sommes avec eux, sauf à accepter de nous penser également comme marchandise industrielle dans le meilleur des mondes possibles.

\footnotetext{
${ }^{11}$ Quand les « libérateurs des animaux » affirment au contraire ne pas aimer les animaux, c'est-à-dire, n'être pas guidés par l'affection dans leurs théories ou leurs actions, il faut prendre également très au sérieux cette position de détachement et de refus du lien.

${ }^{12}$ Il n'est pas nécessaire « d'interpréter une action comme la conséquence d'un exercice ou d'une faculté psychique plus haute si elle peut être interprétée comme l'aboutissement d'une faculté qui est située plus bas dans l'échelle psychologique» (Mc Farland, 1990, p. 459).
} 


\section{Chapitre 13}

\section{Des frontières familières à l'épreuve d'une expérimentation de contraception animale L'« affaire » des marmottes de Prapic}

Isabelle Mauz, CÉline Granjou

Au printemps 2004, le parc national des Écrins lance une expérimentation de contraception des marmottes sur le plateau de Charnières, en zone centrale du parc, dans le but de trouver une solution " innovante et rationnelle " au vieux problème de cohabitation entre les agriculteurs du village voisin de Prapic, qui exploitent le plateau de Charnières, et la population de marmottes qui y prospère. L'expérimentation concerne quelques hectares, deux agriculteurs, une trentaine de marmottes auxquelles des contraceptifs ont été administrés une fois. Dans les mois qui suivent, elle donne lieu à quelques articles dans la presse et suscite des réactions au Conseil national de protection de la nature (CNPN) et au conseil scientifique du parc, qui demeurent limitées. Si l' "affaire » est donc minuscule, elle mérite bien que l'on s'y arrête. Elle semble révélatrice, en effet, d'un certain nombre de changements à l'œuvre dans la façon de considérer et de traiter la nature en général, et les animaux en particulier.

Une vingtaine d'entretiens de type semi-directif ont été réalisés au printemps 2005 auprès des agriculteurs exploitant le plateau de Charnières, des personnes directement impliquées dans l'expérimentation (désignées comme les expérimentateurs), des agents du secteur, de membres du CNPN et du conseil scientifique, enfin de représentants des milieux agricoles, scientifiques, touristiques, cynégétiques et politiques. Les entretiens ont été complétés par des observations réalisées au cours d'un comptage de marmottes et d'une séance de capture, ainsi que par l'exploitation de documents (comptes rendus, articles de presse, rapports de stage).

Selon plusieurs interlocuteurs, les critiques adressées à l'expérimentation s'expliqueraient avant tout par un problème - excès ou déficit - de communication. Celle-ci paraît en effet avoir pesé mais il semble que les réactions observées aient d'autres origines et 
que la remise en cause par l'expérimentation de plusieurs frontières familières ait en particulier posé problème. On montrera ainsi que l'expérimentation de contraception, parce qu'elle relève d'une tentative de dépassement de l'affrontement habituel entre tenants de l'exploitation et protecteurs de la nature, a contrarié les uns et les autres. La technique de contraception employée contribue par ailleurs à l'ébranlement de deux grandes dichotomies établies au sein des êtres vivants : celle qui oppose le sauvage au domestique et celle qui oppose la nature à l'artifice. L'expérimentation amène enfin une partie des personnes rencontrées à s'interroger sur la délimitation, à l'intérieur d'un espace protégé, d'un quasi laboratoire de recherches et sur le statut qui devrait être conféré aux animaux sauvages dans un parc national : c'est cette fois le franchissement de la frontière entre un animal sujet et un animal objet de connaissances qui est reproché aux expérimentateurs.

Avant d'examiner comment l'expérimentation a mis à l'épreuve ces différentes frontières, il faut commencer par fournir les éléments chronologiques les plus marquants et par retracer brièvement le mouvement ayant mené à la décision de lancer l'expérimentation et à sa mise en œuvre.

L'expérimentation s'insère dans une histoire relativement longue, au cours de laquelle quatre périodes principales de régulation des marmottes peuvent être distinguées, qui diffèrent à la fois par les objectifs visés, les acteurs mobilisés et les techniques employées. Les marmottes sont dans un premier temps régulées par les agriculteurs, qui recourent au piégeage, au tir et aux chiens. La création du parc national, en mars 1973, met un terme à ces pratiques (Période 1). Au cours des années 80 , les agents du secteur, confrontés aux doléances croissantes des agriculteurs, entreprennent de capturer et de déplacer des animaux vers d'autres massifs (Période 2). À partir du début des années 90 et pendant plusieurs années, des scientifiques lyonnais mènent des recherches approfondies sur les marmottes du plateau; les translocations sont abandonnées et des discussions sont organisées auxquelles participent l'ensemble des acteurs impliqués (Période 3). Après avoir constaté une augmentation du nombre de marmottons, et convaincus de l'impossibilité de répéter les pratiques antérieures, un petit groupe de personnes conçoivent progressivement, puis mettent en œuvre l'expérimentation de contraception, avec l'aval du conseil scientifique du parc (Période 4).

\section{L'expérimentation met à mal l'opposition entre protection et exploitation de la nature}

L'expérimentation de contraception et les réactions qui l'ont suivie révèlent l'existence de trois conceptions concurrentes de la nature. Deux d'entre elles s'affrontent dans les Alpes depuis plusieurs décennies : l'exploitation des ressources naturelles et la protection de la nature. Nous présenterons l'une et l'autre, dans le cas particulier du plateau de Charnières, et montrerons qu'elles se ressemblent davantage que leur opposition ne le donne à penser. Nous verrons ensuite que l'expérimentation de contraception participe d'une troisième conception, apparue avec le concept de biodiversité, qui entend offrir une issue à l'alternative entre exploitation et protection. 


\section{La vieille rivalité entre exploitation et protection de la nature}

Les montagnards ont longtemps cherché à tirer le maximum de leur environnement. Cela ne signifie pas qu'ils en aient tiré beaucoup, ni qu'ils n'aient pas fixé de limites à leurs prélèvements, ni qu'ils n'aient pas éprouvé d'émotions esthétiques ou un attachement profond à leur environnement. Mais la maximalisation de la productivité était pour eux la norme de l'action. Par nécessité.

\section{Exploitation et productivité}

Dans une conception où la nature doit pourvoir aux besoins et « rendre » à hauteur des efforts consentis, le plateau de Charnières est particulièrement prisé. C'est, en effet, un des rares endroits plats. Situé à quelques minutes de marche du village de Prapic, il est par ailleurs facile d'accès. Il constitue en bref un des meilleurs terrains.

« Ça [les marmottes] nous abîme un peu les quatre jolies prairies qu'on a quoi » (un agriculteur).

Il a aussi constamment joué un rôle majeur dans l'alimentation des hommes et du bétail. Les agriculteurs n'ont eu de cesse d'améliorer encore ces terrains favorables, de les «mettre en valeur », par un travail assidu d'épierrage, de fumure, de lutte contre les adventices... et les marmottes. Car elles aussi apprécient le plateau, où elles trouvent notamment un sol facile à fouir et une flore riche et variée.

«C'est vrai que chez nous, c'est un terrain à marmottes, elles se multiplient très vite » (un agriculteur).

Les marmottes font partie de la fraction de la nature qui, n'ayant pas été domestiquée $^{1}$, ne doit pas entraver l'agriculture ni l'élevage (Dalla Bernardina, 1988, pp. 165185). Mais elles font partie du sauvage exploitable et, en bien des endroits, elles n'ont pas peu contribué à la vie des montagnards, et des bergers en particulier, qui les chassaient pour leur viande, pour leur peau et pour leur graisse ${ }^{2}$. Les gens parvenaient en outre à les «tenir », en recourant à différentes techniques : chiens, piégeage, tir. Les marmottes présentent enfin l'avantage de dormir une bonne partie de l'année, de ne causer alors aucun problème et d'être à l'automne relativement faciles à capturer. Présentant un intérêt certain pour les montagnards et pouvant être assez efficacement maintenues à l'écart des terrains cultivés, où leur présence devient gênante, les marmottes ont été sévèrement contrôlées, et leurs populations fortement réduites, mais elles n'ont pas été éradiquées, contrairement à d'autres espèces sauvages, moins profitables et plus difficiles à contenir (prédateurs notamment).

\section{Protection et naturalité}

Le plateau de Charnières, les agriculteurs, et les marmottes sont considérés tout autrement par les partisans de la protection stricte du plateau. Dans son article 5, le décret de création du parc stipule que « les activités agricoles, pastorales et forestières continuent à être librement exercées, dès lors qu'elles se conforment au présent décret $[\ldots]$ ». Il n’a

\footnotetext{
${ }^{1}$ On sait cependant que des marmottons étaient capturés vivants et apprivoisés. Un des agriculteurs, propriétaire d'un camping à la ferme, détient d'ailleurs, au village de Prapic, quelques marmottes dans un enclos, que les touristes peuvent voir sans monter jusqu'au plateau de Charnières.

${ }^{2}$ À laquelle des vertus thérapeutiques étaient attribuées.
} 
donc jamais été question de prohiber ni même de réduire les activités dites traditionnelles dans le parc.

Cependant, pour certains, que l'on peut qualifier de protecteurs stricts, la création du parc a marqué la reconnaissance d'une conception de la nature qui semble antagoniste de la précédente. Cette fois, en effet, c'est la maximalisation de la naturalité qui doit servir de norme à l'action. Or, la naturalité est assimilée à l'inhumanité et est opposée à l'artificialité qu'induit l'intervention humaine.

Dans cette conception, le parc fait figure de sanctuaire qu'il faut protéger contre les entreprises humaines, y compris agricoles. La présence des hommes devient une concession conditionnelle, suspendue à leur discrétion et à leur modération.

«Alors le débat est très simple et ça c'est important, on en a discuté avec mes amis : soit les gens qui sont dans les parcs nationaux, qui font des activités, ils le font sur la pointe des pieds, en se faisant tout petits et on continuera d'accepter cette exception française où on a certaines activités à l'intérieur des parcs, soit ils veulent avoir les mêmes avantages que leurs collègues qui sont hors parc et c'est complètement impossible à les leur donner. Et à ce moment-là on va arriver à un point où on va faire une pétition en disant: "virons tous les gens qui sont dans les parcs nationaux !!!” C'est ce qu'ils veulent ! ?» (un membre du CNPN).

Non seulement le plateau de Charnières n'est plus autant valorisé que dans une logique d'exploitation mais il pose problème, en raison de son anthropisation marquée. Quant aux marmottes, elles sont désormais considérées comme les victimes d'un combat inégal entre l'homme et la nature.

«On découvre un jour que dans un parc national, la compétition entre les vaches, qui n'ont rien à y foutre, et les marmottes, qui sont faites pour y être, eh bien c'est au détriment des marmottes, parce que l'affaire des marmottes, c'est une compétition, dans un parc national, entre les vaches et les marmottes! » (un protecteur).

\section{Deux conceptions opposées, mais à certains égards convergentes}

L'exploitation des ressources naturelles et la protection de la nature ne peuvent que s'opposer et la seconde s'est d'ailleurs constituée en réaction à la première. Un gain de productivité passe a priori par un contrôle accru de la faune et de la flore, donc par une perte de naturalité. De la même façon, une augmentation de la naturalité signifie moins d'interventions humaines, ce qui a de bonnes chances de se traduire par une réduction des rendements. Dans un tel schéma agonistique, on ne peut à la fois favoriser la productivité et la naturalité, ni contenter les exploitants et les protecteurs, si bien que le traitement réservé aux marmottes du plateau de Charnières devient un moyen de mesurer un rapport de force et la proximité du parc aux uns et aux autres. Laisser les marmottes évoluer, ou au contraire contrôler leurs populations, est interprété comme le signe d'une inclination en faveur des protecteurs, ou des agriculteurs : le parc, érigé en arbitre, est toujours suspecté de partialité par l'une au moins des parties.

Cette rivalité entre protection et exploitation est manifeste et elle est régulièrement soulignée. Leurs convergences le sont moins. Pourtant, l'une et l'autre encouragent un partage des êtres vivants en deux ensembles contraires. Le domestique s'oppose au sauvage pour les partisans de l'exploitation, comme la nature s'oppose à l'artifice pour ceux de la protection : les deux conceptions sont également dualistes. Dans son commentaire de 1'article de Philippe Descola (2004, pp. 17-39) sur le sauvage et le domestique, Daniel Fabre (2005, pp. 175-176) souligne que la séparation entre le sauvage et le 
domestique relève davantage de la limite que de la frontière : il y a toujours eu des passages de l'un à l'autre, et il en va de même entre la nature et l'artifice. Il n'empêche que l'on s'est efforcé, avec plus ou moins de succès, de maintenir ces ensembles distincts et de contrôler les mélanges, essentiellement en partageant l'espace : les animaux ont une place où il leur faut demeurer, sous peine de sanction (Mauz, 2002, pp. 129-145). Le plateau de Charnières, inclus dans l'espace domestique et dans l'espace naturel, semble dès lors voué à un affrontement périodique. Enfin, la protection comme l'exploitation de la nature favorisent les éthiques dites déontologiques, dans lesquelles l'action est évaluée a priori, indépendamment de ses conséquences effectives. Ainsi, toute intervention humaine est nécessairement néfaste dans une conception de la protection pure. On peut éventuellement tolérer les agriculteurs dans les espaces protégés mais l'idéal serait qu'il n'y en ait pas et ils doivent donc se «tenir à carreau ». Il en va de même avec les animaux sauvages dans les terrains exploités.

\section{Gestion et biodiversité}

Une autre conception émerge depuis le début des années 1990, dans laquelle la nature ne s'exploite ni ne se protège : elle se gère, la maximalisation de la biodiversité étant cette fois érigée en norme positive de l'action (Larrère, 1997, pp. 79-92).

\section{L'adoption de la biodiversité comme norme}

En quinze ans à peine, la notion de biodiversité a connu une diffusion remarquable. Bien sûr parmi les scientifiques, mais aussi au sein des espaces protégés. De manière générale, on ne trouve guère aujourd'hui de porte-parole d'usagers des milieux naturels qui ne revendiquent un rôle dans la préservation de la biodiversité ou qui, à tout le moins, n'affirment ne pas lui nuire.

L'accroissement de la productivité et celui de la biodiversité sont clairement des objectifs distincts : le maximum de productivité ne correspond à peu près jamais au maximum de biodiversité. Mais celui-ci ne correspond pas toujours non plus au maximum de naturalité. La biodiversité inclut, en effet, la diversité d'origine anthropique et l'on reconnaît que l'action humaine peut, dans certains cas, accroître la biodiversité. Des prairies d'altitude sont ainsi réputées abriter une flore et une faune plus riches que les landes et les forêts qui les remplaceraient progressivement en l'absence de pratiques agropastorales.

«Limiter la marmotte, c'est le maintien d'un pré de fauche, d'un paysage qui est quand même chouette, si vous connaissez, un paysage qui est agréable, surtout au mois de juin avec toutes les fleurs. Tout ce qui est associé à cette flore diversifiée des prés de fauche, quand on met en balance les deux, je dirais le conseil scientifique a pris la décision en toute connaissance de cause : il avait le choix, il avait le choix de se caler sur un principe je dirais un peu fantasmatique d'une nature vierge à évolution spontanée, qui ne correspond pas à la réalité de terrain puisqu'on est dans un milieu qui a été créé et qui est entretenu par l'homme et qui a une diversité biologique, et nous, notre rôle au conseil scientifique c'est d'essayer de faire en sorte qu'il y ait la plus grande diversité biologique » (un membre du conseil scientifique).

L'adoption de la biodiversité comme norme s'accompagne de toute une série de changements. L'espèce cède la place à la population (Micoud, 1989). On ne cherche pas à exercer une action sur les marmottes en général, en tant qu'espèce, mais uniquement sur la population du plateau de Charnières et même, plus précisément, sur certains groupes familiaux considérés comme des groupes « à problèmes ». 
« On s'est tourné vers $\mathrm{X}$, un petit peu en disant : dis donc, on a un problème, il faudrait..., ça sert à rien d'en capturer, on l'a déjà fait, c'est quelques groupes familiaux, qu'est-ce qu'on pourrait faire? » (un expérimentateur).

Une logique de type conséquentialiste prend par ailleurs le pas sur les logiques déontologiques : on se garde de se prononcer d'emblée, par principe, sur le bien-fondé d'une action ou d'une situation, attendant d'en examiner les effets pour décider si elles sont bonnes ou mauvaises et donc si elles doivent être poursuivies ou interrompues. Une intervention pouvant être favorable ou défavorable à la biodiversité (alors que, du point de vue des protecteurs stricts, elle ne peut que nuire à la naturalité), l'arrivée ou le départ d'une espèce sauvage, l'augmentation ou la diminution d'une population pouvant également être fastes ou néfastes, il importe de juger au cas par cas, en fonction des circonstances singulières dans lesquelles on se trouve, et de déterminer ensuite, sur pièces, ce que l'on doit faire : on est dans le « registre de la permission » (Larrère, 2005).

Enfin, l'adoption de la biodiversité comme norme remet en cause les partages inhérents aux conceptions antérieures. L'objectif étant qu'un milieu héberge la plus grande diversité possible, on s'extrait de systèmes où les êtres vivants sont partagés en deux blocs opposés - le sauvage et le domestique, la nature et l'artifice -, qu'il faut à tout prix maintenir à distance, en évitant les mélanges, synonymes de désordres à venir. Il est vrai que toutes les espèces ne sont pas regardées comme équivalentes et que certaines se voient accorder un statut particulier, soit parce qu'elles sont censées être de bons indicateurs d'une biodiversité que l'on ne sait toujours pas mesurer (exemple des espèces dites « parapluies »), soit parce qu'elles sont distinguées en raison de l'intérêt que leur portent les humains (espèces remarquables, phares, emblématiques, patrimoniales, etc.). Il est vrai aussi que certains tendent à recréer des clivages au sein du vivant, en distinguant une bonne et une mauvaise biodiversité (Mauz et Rémy, 2004, pp. 295-306). Il n’en reste pas moins que la biodiversité s'accompagne plutôt d'une progression graduelle, du moins diversifié au plus diversifié, que d'une limite entre des catégories opposées, et qu'elle est de ce fait moins marquée par l'obsession de la pureté que la recherche de la naturalité et de la productivité. En même temps qu'elle ébranle les catégories communément admises, la biodiversité remet en cause les zonages de l'espace. Il apparaît possible, et souhaitable, de l'accroître partout, dans les espaces que l'on protège comme dans ceux que l'on exploite : « cette notion entérine ainsi qu'il n'y a pas de frontières entre milieux naturels et espaces de mise en valeur » (Larrère, 1997, p. 79).

\section{Les critiques de l'approche "gestion de la biodiversité »}

La gestion de la nature au nom de la biodiversité n'a clairement pas fait disparaître les conceptions antérieures. L'un des agriculteurs a déposé en 2005 une demande d'irrigation du plateau, dans le but d'augmenter sa productivité. Des protecteurs continuent de leur côté de considérer la présence d'agriculteurs comme un obstacle à surmonter pour atteindre leur idéal de naturalité.

La recherche de la biodiversité maximale contrarie les irréductibles de la protection comme ceux de l'exploitation. Elle suppose en effet d'exercer un contrôle et une discipline sur les humains et sur les non-humains, tandis que la protection et l'exploitation font peser des contraintes sur une partie seulement des êtres vivants. Dans la protection, en effet, seuls les hommes sont tenus de respecter un ensemble de règles et sont surveillés, contrôlés et éventuellement sanctionnés. Tout ce qui arrive naturellement doit en revanche être accepté 
comme normal : « Dans la nature "pure” ainsi redéfinie, tout élément, pour autant qu'il est naturel, ne peut être que "bon". Il ne peut pas y avoir d'animal naturel nuisible, il ne peut y avoir que des nuisances, c'est-à-dire des agressions anthropogènes contre les milieux » (Micoud, 1989, p. 40). Aussi les êtres naturels doivent-ils échapper à toute intervention qui viendrait restreindre leur part de liberté. Dans l'exploitation, il existe bien des règles d'usage des milieux, parfois très strictes, et l'on connaît le caractère tatillon des anciens règlements d'alpage, où tout - le nombre d'animaux locaux et étrangers pouvant être inalpés, la date d'inalpage et de désalpage, etc. - était soigneusement fixé et vérifié. Mais, outre que ces règles sont largement tombées en désuétude avec la disparition des communautés agropastorales, elles visaient moins à réduire l'exploitation humaine des milieux qu'à garantir une relative équité entre les montagnards et à éviter l'appropriation, par une minorité, de ressources indispensables à tous. S'il importe de répartir les ressources entre les montagnards, il n'est pas question de les partager avec d'autres espèces. Pas de quartier pour les animaux sauvages, donc, dès lors qu'ils enfreignent les limites du domestique. L'homme entend se réserver le monopole de l'exploitation de la nature, partout où elle est exploitable.

Si l'adoption de la biodiversité permet d'affirmer l'intérêt des activités agropastorales, qui cessent d'être tout juste tolérées, elle ne dispense pas les agriculteurs de rendre des comptes. Bien au contraire, ils doivent désormais apporter la preuve qu'ils ne nuisent pas à la biodiversité ni à la qualité générale des milieux et même qu'ils les favorisent. La gestion de la biodiversité constitue probablement un progrès par rapport à une situation où c'est la naturalité qui primait mais elle ne restaure pas pour autant une situation où c'est la production qui l'emportait.

Les protecteurs de la nature sont dans l'ensemble plus virulents que les agriculteurs à l'encontre de l'expérimentation. Ce n'est pas surprenant : l'idée selon laquelle la nature pourrait être gérée, y compris dans les espaces protégés, constitue un demi-progrès pour les agriculteurs, mais une régression difficilement acceptable pour les protecteurs, puisqu'elle implique d'étendre à la nature une pression jusque-là réservée aux hommes et aux êtres qu'ils ont artificialisés. L'expérimentation nécessite un suivi individualisé des animaux. Elle repose sur toute une instrumentation, ainsi que sur la désignation de groupes familiaux «à problèmes » (parce que colonisateurs), que l'on s'autorise à traiter. Elle s'inscrit ainsi dans un processus d'extension à l'ensemble du vivant des procédés « disciplinaires » (Foucault, 1975), qui marque la fin de la nature aux yeux des protecteurs.

Les protecteurs purs en veulent d'autant plus aux expérimentateurs qu'ils en sont proches. Ils admettent mal que l'on cherche à diminuer une population de marmottes, après s'être fortement impliqué dans la protection d'espèces menacées. Ils voient là un revirement, pour ne pas dire une trahison inexplicable.

« $\mathrm{X}$, que tu connais, à un congrès sur la faune sauvage où $\mathrm{y}$ avait $\mathrm{Y}$ et il $[\mathrm{X}]$ l'a pris [Y] à partie en disant : "mais écoute, là j'ai entendu parler de ce que fait $Z$ [un expérimentateur], là", ouais ouais il était choqué » (l'expérimentateur en question).

De manière générale, il n'est pas facile, pour des personnes depuis longtemps investies dans la protection de la nature, d'accepter l'idée de réguler des populations animales sauvages, de pratiquer, en quelque sorte, une « éco-zoo-technie (Micoud, 1989) négative ». Inversement, les acteurs défendant la conception gestionnaire qualifient leur propre camp de moderne et placent les protecteurs purs dans le camp des «vieux 
schnocks ${ }^{3} »$, partisans d'une vision de la nature dépassée. Les scientifiques, bien souvent, préfèrent s'intéresser aux espèces menacées ou rares, qu'il faut protéger, qu'à celles, localement surabondantes, qui se distinguent par les problèmes qu'elles causent. Outre que la définition de la surabondance est toujours discutable, admettre l'existence de problèmes implique de rechercher des moyens de contenir les populations ; or, nombre de scientifiques et de protecteurs estiment que ce n'est pas là leur rôle (Garrott et al., 1993, pp. 946-949). Que la marmotte ne soit pas une espèce introduite, comme le ragondin et le rat musqué ${ }^{4}$, mais au contraire une espèce typiquement alpine, n'arrange rien : si la régulation d'une espèce exotique est acceptée voire réclamée, celle d'une espèce autochtone et qui plus est emblématique semble aux protecteurs particulièrement scandaleuse et déplacée.

Ainsi, le principe de l'expérimentation, qui vise à dépasser l'opposition jusque-là dominante entre protection et exploitation, mécontente profondément les protecteurs sans pour autant satisfaire les exploitants. Il séduit en revanche les partisans de la conciliation, parmi lesquels on trouve à la fois des agents du parc, des scientifiques, et des acteurs locaux, tous las de la confrontation entre protection et exploitation et désireux de lui trouver, enfin, une issue.

\section{La contraception ébranle les catégorisations traditionnelles des animaux}

Considérons à présent la technique de contraception elle-même. En administrant des substances contraceptives à des animaux sauvages, on intervient dans leur cycle de reproduction, ce qui est le propre du médecin, ou de l'éleveur. On applique donc au sauvage un procédé jusque-là réservé à l'homme et à ses animaux, brouillant une frontière déjà beaucoup moins nette que par le passé, du fait de l'augmentation et de la diversification de la grande faune sauvage et de l'évolution du comportement des animaux domestiques et sauvages (Mauz, 2005). Il y a là un désordre qui surprend.

«Surtout venant du parc, ça [1'expérimentation de contraception] les a étonnés quoi [les chasseurs]. Parce qu'ils ont fait la comparaison par rapport à l'acte de chasse quoi : on leur interdit de chasser dans la zone centrale, de prélever des animaux et maintenant on veut les empêcher de s'y reproduire dans cette zone-là et on leur explique que c'est une zone de protection donc c'est vrai que... ça... disons ça a mal été compris pour ça » (un représentant des milieux cynégétiques).

Il inquiète aussi, tant le désordre semble porteur de risques.

« Ça va faire [...] des grosses marmottes bien grasses qui vont perdre la vie, qui vont perdre leurs qualités $[. .$.$] qui vont perdre leur vie, ça va plus être des marmottes. C'est comme la$ maladie du chamois, tout ça : c'était pas chez nous, avant» (un agriculteur).

Le procédé ne sied pas davantage aux protecteurs stricts, puisque l'on recourt à un artifice à l'encontre d'animaux naturels, effaçant là aussi les frontières, au lieu de s'appliquer à les renforcer. Le risque souligné est alors celui d'une extension généralisée des interventions sur la reproduction pour gérer les problèmes de surabondance comme de rareté de certains animaux.

\footnotetext{
${ }^{3}$ Selon l'expression d'un membre du CNPN.

${ }^{4} \mathrm{Si}$ les méthodes de contrôle de ces deux espèces, importées respectivement d'Amérique du Sud et du Nord, évoluent (Mougenot et Roussel, 2005), le bien fondé de leur régulation, lui, n'est guère contesté.
} 
«Tant qu'on y est, maintenant on a lancé le truc, on pourrait peut-être cloner les ours pour qu'on en ait... Il reste un mâle, on pourrait peut-être faire des femelles avec, enfin, on est partis dans une folie, dans un parc national où tout devrait se faire naturellement » (un membre du CNPN).

Beaucoup préféreraient le tir à la contraception, ou l'emploi de chiens, parce que le tir et les chiens sont les instruments de la chasse et que les animaux sauvages doivent être chassés.

«J'oserais dire largement que je reconnais à la chasse plus de naturel que la contraception » (un responsable agricole).

La non-intervention constitue pour les protecteurs la meilleure solution mais ils préfèrent eux aussi recourir au tir, bien qu'ils n'y soient certainement pas favorables, parce que le tir se rapproche tout de même davantage de l'action que pourraient avoir des prédateurs. Ainsi, pour les protecteurs purs comme pour les exploitants, la contraception est une technique qui, plus que le tir, déconstruit leur conception de la nature, pour en introduire une autre, celle d'une nature destinée à être, de plus en plus, gérée.

«Si il y a des débats aussi forts sur ce sujet-là, c'est bien qu'y a une espèce d'inquiétude sur la marge entre le sauvage donc qui représente le naturel, et puis le en gros géré par l'homme et là on rejoint tout l'univers du fantasme de : qu'est-ce qui est encore naturel aujourd'hui et qu'est-ce qui est manipulé ? Et je crois que le cœur du problème il est là. Et nous on représente l'univers de la garantie du naturel, du sauvage, du wilderness, des valeurs abstraites, non marchandes, etc. etc., et quand on fait ce type de choses, on quitte cet univers-là pour rejoindre celui de l'interventionnisme, du scientifique alors tout ça se mélangeant avec tout quoi, le débat sur les OGM, le problème de la vache folle, enfin tout ça ça constitue un espèce de grand univers je suis sûr de la manipulation en gros, manipulation pouvant s'entendre d'ailleurs dans toutes acceptions » (un agent du parc).

Le passage d'un système où des individus sont prélevés et déplacés, c'est-à-dire physiquement supprimés, à un système qui s'efforce de gérer la vie, n'est pas sans rappeler l'émergence d'un «bio-pouvoir» que Michel Foucault a décrite à propos de l'histoire de la sexualité humaine : le pouvoir ne s'exerce plus au moyen d'un prélèvement pur et simple, mais à travers une gestion de la population qui s'appuie sur des techniques scientifiques d'observation et de contrôle (Foucault, 1976, p. 178). L'extension du bio-pouvoir à des populations animales sauvages, que l'on peut qualifier, avec Lascoumes, d' " écopouvoir » (Lascoumes, 1994, p. 312), soulève manifestement des interrogations.

\section{Des frontières du parc à celles du laboratoire: quel statut pour les marmottes?}

Nous soulignerons ici la manière dont la visée de recherche scientifique qui sous-tend l'opération de contraception instaure un certain nombre de frontières, qui interfèrent avec les frontières propres au parc, notamment en ce qui concerne le statut conféré aux marmottes. Nous montrerons d'abord que la démarche expérimentale institue des frontières à l'intérieur même de celles du parc, en délimitant un périmètre voué à l'expérimentation. Nous verrons alors que le statut d'objet de connaissances qu'acquièrent les marmottes à l'intérieur de ce périmètre paraît difficilement conciliable, pour différentes raisons, avec l'exclusion de l'instrumentalisation de la nature par l'homme au sein du parc. 


\section{Le périmètre expérimental :}

\section{un laboratoire à l'intérieur des frontières du parc ?}

L'opération de contraception est conçue par ses initiateurs sur le mode d'une expérimentation : elle vise à tester une technique, observer ses résultats et en tirer un bilan quant à son efficacité pratique (possibilité de réguler les problèmes posés par les marmottes) et à sa portée théorique (connaissances éthologiques).

« Ça fait partie d'une démarche je dirais normale pour un scientifique, il y a un problème à résoudre, on essaie les différentes possibilités, les possibilités précédentes, ce qu'on a essayé n'a pas marché : il y a une autre solution eh ben on essaie autre chose, c'est tout. Et puis on fera le bilan et on verra si ça marche ou pas, ce qui conduira ou non, soit à pérenniser, soit encore à chercher autre chose. Je dirais c'est dans le cadre d'une démarche rationnelle » (un membre du conseil scientifique).

Pour assurer l'efficacité de cet objectif d'évaluation (suivi précis, possibilité de comparaison), les expérimentateurs opèrent sur un nombre d'animaux réduits, sur un espace délimité, avec un protocole défini et limité dans le temps. L'expérimentation a lieu sur le plateau de Charnières et plus précisément sur deux zones de ce plateau, correspondant à une trentaine de marmottes (cibles). Pour les expérimentateurs, il s'agit d'une démarche localisée et de petite échelle (une trentaine de marmottes sur quelques hectares).

«Et tout ça évidemment sur une zone restreinte : c'était pas une contraception pour toutes les marmottes de la montagne! » (un expérimentateur).

La contraception dure une saison et les effets seront observés pendant l'été (puis le protocole de suivi sera élargi à l'été suivant). Le caractère réversible de la contraception - par rapport à une stérilisation - est souligné.

«Plus une volonté d'expérience et d'évaluation, c'est-à-dire avec un protocole qui était réversible, puisque en fait ça coupe juste la saison de chaleur, un protocole qui était limité aussi dans l'espace puisque en fait les territoires qui ont été traités ça représente $10 \%$ de la zone » (un expérimentateur).

Enfin, le protocole expérimental délimite également les types d'impact possibles de la contraception, en désignant les éléments qui vont être observés et évalués afin de faire le bilan de l'opération: le nombre de marmottons qui vont naître, mais aussi certains comportements des marmottes, que l'on estime susceptibles d'être modifiés par la contraception.

«On avait établi avec $\mathrm{X}$ toute une liste de comportements qui étaient à relever par les stagiaires, s'il y avait du jeu, s'il y avait des combats, s'il y avait des séances d'épouillement » (un membre du conseil scientifique).

Le périmètre expérimental fonctionne ainsi comme une « zone franche » à l'intérieur du parc, instaurant ses propres frontières et ses propres objectifs, à la manière d'un laboratoire clos sur lui-même. Or c'est précisément la possibilité d'une étanchéité entre les frontières du « laboratoire » et le reste du territoire du parc qui est mise en doute ou niée par un certain nombre de voix critiques.

Beaucoup d'acteurs s'inquiètent d'abord des effets secondaires possibles de la contraception, mettant ainsi en question de manière très concrète cette étanchéité entre le périmètre de l'expérimentation et le reste du parc : pour eux, l'opération pourrait s'avérer nettement moins délimitée quant à sa cible et ses impacts, que ne le prétendent les expérimentateurs. 
«Le parc ne doit jamais oublier, et y compris les scientifiques qui travaillent sur des programmes comme ça ne doivent jamais oublier qu'ils font pas des expériences en laboratoire, ils font des expériences dans un territoire... » (un membre du conseil scientifique).

Ils soulèvent ainsi le risque de disséminer le produit chimique contraceptif dans le milieu.

« On agit sur la reproduction et là si ce sont des actions qui sont pas trop maîtrisées, on peut, on peut se poser des questions des questions sur des effets incontrôlés qui pourraient en découler. On a quand même des exemples, on a quand même des exemples importants, notamment sur... on en a eu un bel qui concernait la myxomatose où effectivement il y a eu des manipulations qui ont été faites en parc et d'un seul coup c'est sorti du parc et ça a envahi toute la France : oui oui, en 52, la myxomatose, elle est sortie d'un parc de Sologne, où il y a un vétérinaire qui faisait des tests sur la vaccination » (un acteur cynégétique).

Ils s'interrogent ensuite sur le risque d'irréversibilité, qui transformerait la contraception en stérilisation durable.

«Donc le risque il est là, c'est de dire est-ce qu'il y aurait pas à un moment donné quelque chose qui fait que ça ne soit plus sous le contrôle de l'homme... Et auquel cas, si on se retrouve à stériliser toutes les marmottes du coin c'est gênant! Et les stériliser une bonne fois pour toutes quoi » (idem).

On constate enfin un questionnement sur les impacts potentiels de la contraception qui élargit le cercle des effets possibles aux autres marmottes ou à d'autres espèces du parc. Les produits contraceptifs pourraient par exemple avoir un impact sur les prédateurs des marmottes.

« La marmotte, elle est dans la chaîne alimentaire une proie et donc il y a beaucoup de prédateurs comme le renard, comme l'aigle, qui s'en nourrissent et donc... bon, c'est vrai je pense disons que c'est pas les effets de la stérilisation localement sur un endroit qui posent question » (un chasseur).

Certaines personnes s'attendent également à des modifications non prévues du comportement ou de l'aspect des marmottes (obésité).

«Non, ça [la contraception] va faire des grosses marmottes bien grasses, qui vont perdre l'équilibre de la vie» (un agriculteur).

L'idée d'instaurer un périmètre expérimental en milieu ouvert, qui plus est à l'intérieur d'un parc national, pose ainsi problème à certains acteurs.

« Soit on dit c'est limité à un endroit donc dans ce cas-là on devient les expérimentateurs et on est associé à tous ces gens qui font des espèces de recherches de laboratoire qui utilisent le vivant pour avancer les connaissances [...] ça pose quand même le problème de : est-ce que les parcs nationaux sont des laboratoires à ciel ouvert, est-ce qu'on y fait de l'expérimentation sur du vivant?» (un agent du parc).

Il s'agit en effet d'un déni de la vocation de protection du parc au profit d'une autre visée, celle de production de connaissances.

«Y a... comment dire ? Y a un primat qui est celui de la connaissance, et la connaissance à tout prix, même si on tue les symboles, personnellement je suis pas du tout d'accord avec ça » (un agent du parc).

Ce sont les conséquences de ce «primat de la connaissance» que nous allons maintenant analyser. 


\section{Production de connaissances et instrumentalisation de l'animal}

L'instauration d'un laboratoire à l'intérieur du parc à fin d'expérimentation et de recherche interroge la hiérarchie des objectifs du parc, notamment dans la mesure où elle s'accompagne de (et justifie) divers mécanismes d'instrumentalisation de l'animal au profit d'enjeux liés à la production de connaissances, au sein d'un territoire dont la vocation première est de préserver la liberté et l'autonomie des animaux sauvages.

Pour plusieurs acteurs, les marmottes permettent la valorisation au premier chef des scientifiques lyonnais, qui ont fait du plateau de Prapic un spot d'études à marmottes, et ont produit une quantité de textes, thèses, publications dans leur propre communauté scientifique sans apporter en retour quoi que ce soit aux habitants du plateau, qu'ils soient humains ou non humains.

«Ça serait intéressant de voir le nombre de publications, de conférences, de rapports de stage, divers et variés, etc. etc. etc. qui ont été faits sur ce sujet-là quoi, $\mathrm{X}$ que vous allez voir la semaine prochaine, il était stagiaire ici y a peut-être vingt ans ou quinze ans, pour capturer des marmottes sur le plateau de Charnières, bon, ç'a été un sujet récurrent, un os à ronger quoi ; [moi : oui, c'est une façon de voir les choses] moi j'aurais tendance à dire : bon, j'veux pas, mais depuis le temps qu'on les étudie, on devrait quand même savoir quoi faire ; ça fait vingt ans » (un agent du parc).

Certaines voix critiques ont même suggéré que l'expérimentation donnait avant tout l'occasion à ses promoteurs (agents du parc ou scientifiques) de s'amuser et de se divertir.

« Moi après je considère [1'expérimentation] comme un jeu scientifique, pourquoi pas, je suis pas contre » (un agent du parc).

Il est clair en tout cas que l'expérimentation permet au parc de se positionner comme expert en matière de faune sauvage, selon un processus où les intérêts propres des animaux apparaissent secondaires par rapport à une certaine affirmation de scientificité et de technicité. Placés devant le choix entre le tir et la contraception, les agents du parc justifient la démarche de contraception par le fait qu'elle s'appuie sur des connaissances pointues acquises récemment (caractère territorial des populations, structures hiérarchiques, attribution des grattis aux jeunes marmottes), qui permet aux expérimentateurs de présenter leur démarche comme " plus adaptée, plus technique et élégante ».

«Y a aussi que si on tire les marmottes, c'est simple, y a pas lieu d'en faire une étude [...]. Lorsqu'on lance une expérimentation de contraception, de marquage, c'est quand même plus innovateur, c'est quand même plus valorisant... » (un agent du parc).

Enfin, on peut considérer que le mécanisme même de la production de connaissances constitue une forme d'instrumentalisation de l'animal, qui n'est plus considéré en soi (sujet de sensations ou d'exigences autonomes) mais vaut en tant que son comportement est source d'observations et de savoir. Si l'image du cobaye vient bien sûr à l'esprit comme archétype de l'attitude d'irrespect envers l'animal, cette notion tend toutefois à faire de l'animal un objet de manipulation à l'infini aux mains des scientifiques. Certaines analyses issues de la nouvelle sociologie des sciences tiennent mieux compte de la « récalcitrance » qu'oppose la réalité au scientifique, et paraissent, en tout cas dans la situation étudiée, mieux refléter le statut donné à la marmotte, de qui on exige de continuer à avoir des comportements autonomes, adaptés à un animal sauvage vivant en liberté. Ces analyses sont basées sur la notion de chaîne d'inscriptions (Latour, 1989) : le scientifique, pour obtenir de la réalité qu'il étudie des réponses aux questions qu'il lui 
pose, met en place tout un dispositif de production d'inscriptions successives caractérisées par une robustesse de plus en plus forte - relevés expérimentaux, graphes, interprétations intermédiaires, rapports, et finalement, publications et ouvrages. Dans notre cas, les marmottes sont dotées d'un système d'identification (bagues auriculaires numérotées, colliers différenciés visibles de loin), qui constitue la base du suivi des populations.

«Surtout ils [les animaux] sont marqués avec une bague pérenne, une toute petite boucle métallique à l'oreille et un signe visible à distance, c'est un collier en fait avec un pendentif, de formes différentes, et ensuite, pendant les trois mois, y a eu tout un travail de suivi pour voir, ben, si les animaux restaient sur site dans leur terrier et si leur place hiérarchique était modifiée » (un expérimentateur).

Tableau 2. Identification individuelle des marmottes lors des captures réalisées en 2005

\begin{tabular}{lccccccc}
\hline & $\begin{array}{c}\text { Zone } \\
\text { de capture }\end{array}$ & $\begin{array}{c}\mathbf{N}^{\circ} \\
\text { de capture }\end{array}$ & Sexe & $\begin{array}{c}\text { Poids } \\
\text { (en kg) }\end{array}$ & $\begin{array}{c}\text { Age } \\
\text { estimé }\end{array}$ & $\begin{array}{c}\text { Boucle } \\
\text { auriculaire }\end{array}$ & Médaille \\
\hline Grincheuse & 3 D & 2 & F & 2,4 & 2 ans & 8824 & $\triangle 545$ \\
Hutch & 3 C & 2 & M & 4 & 3 ans & 8603 & O 502 \\
Crevette & 3 E & 2 & F & 2,8 & 2 ans & 8779 & L 503 \\
Play-Boy & 3 E & 2 & M & 2,9 & 2 ans & 8729 & T 504 \\
Mégère & 3 E & 1 & F & 3,3 & 3 ans & 8605 & $\square 505$ \\
Flocon & 3 E & 2 & M & 3,5 & 3 ans & 8506 & $\Delta 506$ \\
Martin & 3 E & 1 & M & 2,7 & 2 ans & 8698 & П 507 \\
Scoubidou & 3 B & 1 & M & 3,4 & 3 ans & 8827 & $\diamond 508$ \\
Josette & 3 B & 1 & F & 3,3 & 3 ans & 8701 & I 509che \\
Tom & 3 B & 1 & M & 3,3 & 3 ans & 8670 & $\perp 510$ \\
Pamella & 3 A & 1 & F & 3,4 & 3 ans & 8671 & П 511 \\
Surprise & 2 B & 2 & F & 3,6 & 2 ans & 8608 & $\square 512$ \\
Truffette & 2 A & 2 & F & 3,1 & 2 ans & 8845 & $\triangle 513$ \\
Martine & 2 B & 1 & F & 2,8 & 2 ans & 8702 & $\diamond 514$ \\
Johnny & 3 B & 1 & M & 2,8 & 2 ans & 8704 & L 515 \\
Mitch & 3 A & 1 & M & 2,7 & 2 ans & 8794 & $\diamond 516$ \\
Bonnemine & 2 C & 1 & F & 4,8 & Adulte & 8795 & $\square 517$ \\
Casse-Bonbon & 2 A & 2 & M & 3,8 & 3 ans & 8739 & I 518 \\
\hline
\end{tabular}

(Kreiss, 2005, p. 48)

Ce dispositif d'identification (tableau 2) est ainsi le premier maillon d'une chaîne d'inscriptions telles que cartes (localisant les terriers et les zones territoriales), tableaux synthétisant les caractéristiques individuelles de sexe, d'âge, de zone d'habitat, de traitement contraceptif reçu, servant de base aux analyses des rapports de stage. La marmotte n'est donc pas un « simple cobaye » dans les mains d'un expérimentateur, mais se trouve ainsi individualisée, nommée et numérotée ${ }^{5}$ non pas pour sa valeur propre, mais bien afin d'être capable de donner les réponses attendues aux questions que lui pose l'expérimentateur : elle vaut en tant que source $d^{\prime}$ ' inscriptions ».

\footnotetext{
${ }^{5}$ Surnom et numéro coexistent sur les tableaux des rapports de stage.
} 
La dimension expérimentale place ainsi l'animal, par ces différents mécanismes, en situation d'instrumentalisation, d'objet servant une visée supérieure (valorisation professionnelle ou production de savoirs), alors que le parc est précisément le lieu où il devrait être respecté et considéré pour lui-même, irréductiblement à tout usage que peut en faire l'homme. Au bout du compte, seule une science purement descriptive, consistant en suivis, en observations et en inventaires, sans visée d'expérimentation, serait compatible avec la vocation du parc.

« Pour nous, un parc national, vu sa faiblesse en superficie devrait être, alors non pas sous cloche mais devrait être le seul lieu où la nature, la faune, la flore, puissent se développer en toute liberté pour permettre une étude dans les meilleures conditions : ça doit être un sanctuaire » (un membre du CNPN).

« On ne met pas en débat une décision de cet ordre, on fait... on fait ça mais avec la meilleure bonne foi, comme on fait un inventaire de chauve-souris ou comme on fait un suivi des oiseaux hivernants ou je ne sais pas quoi enfin » (un agent du parc).

\section{Conclusion}

L'expérimentation de contraception des marmottes de Prapic constitue assurément une toute petite affaire, mais c'est une affaire exemplaire en ce sens qu'elle provoque brouillages et interférences entre différents rapports à l'animal.

L'expérimentation de contraception interroge les frontières géographiques et déontologiques du parc, en instaurant une sorte de laboratoire à l'intérieur de son territoire, le périmètre expérimental, destiné à tester et affiner de nouveaux savoirs et savoir-faire en matière de gestion de la faune sauvage. À l'intérieur de ce périmètre, l'animal devient l'instrument et l'objet d'une visée de production de connaissances (inséparable de certains intérêts sociaux d'affirmation ou de valorisation professionnelle), qui paraît à certains inconciliable avec le statut de sujet que le parc doit précisément donner à l'animal.

Elle brouille ainsi les frontières sauvage/domestique et naturel/artificiel, constitutives de deux grands types de rapports de l'homme à l'animal liés respectivement au « monde » de l'exploitation des ressources naturelles et à celui de la protection de l'environnement.

Elle témoigne finalement de l'émergence d'une approche résolument gestionnaire des milieux et des animaux, qui, au nom de la préservation de la biodiversité et des paysages remarquables, se démarque tant de l'idée d'exploitation des ressources naturelles que de l'idée de protection pure. À travers des interventions élargies sur le milieu, cette approche gestionnaire vise non plus seulement la sauvegarde d'espèces menacées et la réintroduction d'animaux disparus, comme par le passé, mais également la surveillance et le contrôle d'espèces qui posent localement problème. Elle est liée à l'affirmation de l'expertise du parc à travers des interventions techniques et pointues sur la faune sauvage, à l'image d'une sorte d' " éco-cratie », qui pose en même temps la question de la vocation de recherche du parc, et de la distance que celle-ci peut induire avec la mission première de protection. Le cas des loups, des sangliers et des campagnols, responsables de dégâts autrement plus importants que les marmottes, a ainsi été régulièrement évoqué dans les entretiens, plusieurs interlocuteurs espérant (et d'autres redoutant) que les marmottes de Prapic constituent un banc d'essai et que, à l'avenir, d'autres populations animales sauvages soient à leur tour régulées par des moyens qui restent largement à inventer. 


\title{
Chapitre 14
}

\section{Espoirs et désespoirs de l'extrapolation des résultats expérimentaux de l'animal à l'homme}

\author{
LUC ROCHETTE
}

Évoquer en termes d'espoirs et de désespoirs l'extrapolation des résultats expérimentaux de l'animal à l'homme demande au préalable de rappeler l'importance des travaux qui ont été réalisés au cours de ces trente dernières années sur ce sujet et qui ont permis de mettre en place de nouvelles approches thérapeutiques. Si l'on s'en réfère aux données bibliographiques, il apparait sur les sites informatiques, par exemple, PubMed, que plus de 4 millions de références rapportent des résultats de travaux chez l'animal, et que plus de 10 millions d'articles relatent des études chez l'homme.

Maintenant regardons les espèces qui servent principalement aux études pharmacologiques. En premier lieu, apparait le rat avec plus d'un million de références, puis la souris avec 900000 références. Quels sont les domaines pharmacologiques qui ont été le plus étudiés au cours de ces dernières années? Dans le domaine cardiovasculaire, il s'agit du diabète, puis des désordres coronariens, de l'insuffisance cardiaque et enfin de l'athérosclérose. En ce qui concerne le cerveau, on trouve, dans l'ordre, la maladie d'Alzheimer, les démences séniles puis les psychoses. Pour le cancer, on distingue, les leucémies, les cancers du sein et du poumon et enfin le cancer de la prostate.

Les modèles animaux sont nombreux, de plus en plus validés (Tableau 3). Ils utilisent des animaux normaux ou des animaux pathologiques. Les animaux pathologiques sont des animaux qui peuvent présenter spontanément une altération des fonctions physiologiques, exemples : les animaux spontanément hypertendus (SHR) ou obèses (Zuker). Ces modèles sont caractérisés par des similitudes avec des pathologies rencontrées chez l'homme comme l'hypertension, l'athérosclérose, les diabètes ou les tumeurs. Il est possible également d'induire par l'introduction de substances des troubles du fonctionnement de certains organes; c'est le cas des traitements des animaux par l'alloxane ou 
la streptozotocine qui engendrent des diabètes. Précisons que, selon les souches d'animaux, des susceptibilités peuvent exister vis-à-vis de la «toxicité » de ces composés. Depuis quelques années, il est possible de réaliser des animaux dits transgéniques ; ces modèles transgéniques constituent une approche particulièrement intéressante dans la connaissance des mécanismes intimes qui sous-tendent l'initiation et le développement de pathologies. Les animaux les plus souvent rencontrés expérimentalement seront donc la souris, le rat, le cobaye, le lapin, le porc, le chat, le chien et, dans des cas plus exceptionnels, les singes.

Tableau 3. Modèles animaux

\begin{tabular}{lll}
\hline \multicolumn{1}{c}{ Normaux } & \multicolumn{1}{c}{ Pathologiques } \\
\hline Souris & Transgéniques & Induction \\
Rat & Zucker - SHR & "Troubles comportementaux » \\
Cobaye & & Hypertension \\
Lapin & & Athérosclérose \\
Porc & & Diabètes \\
& & Tumeurs \\
\hline
\end{tabular}

Bien entendu, il faut tout de suite faire cette distinction entre le règne animal et le comportement humain, aussi bien sur le plan biochimique que sur le plan comportemental. Si l'on compare ces espèces, on sait que les durées de vie sont très variables : plus l'animal est petit, plus son espérance de vie est courte, et plus, sur le plan cardiovasculaire, sa fréquence cardiaque est élevée. Le comportement est bien entendu un sujet de recherche et a été l'objet de très nombreuses études durant le siècle dernier ; ce qui a permis la découverte des médications du type psychotrope. Dans ce cadre, il existe de nombreuses approches expérimentales utilisant des appareils, reliés maintenant à des systèmes informatiques, qui permettent de préciser au cours du nycthémère (alternance jour/nuit) l'activité exploratrice d'animaux placés dans des environnements, qui peuvent être nouveaux et éventuellement stressants. Dans le cadre du comportement, il est bien entendu, tout à fait important de rappeler le comportement maternel, qui présente des spécificités dans chaque espèce.

Le développement de l'expérimentation animale au cours de ces dernières années a été codifié et des comités d'éthiques d'expérimentations animales ont été mis en place au niveau de chaque centre de recherche. Il existe également des comités d'éthique pour les essais cliniques qui sont régis par des règles très strictes.

Sur le plan historique, il est intéressant de constater des « rencontres », certainement non fortuites, entres des découvertes scientifiques et les courants littéraires et artistiques. J'en veux pour preuve, en décembre 1962, lors de la remise des prix Nobel, la rencontre des trois Prix Nobel découvreurs de la structure de l'ADN : James Watson, Francis Crick et Maurice Wilkins avec John Steinbeck qui recevait le Prix Nobel de littérature. On se souvient que Steinbeck, auteur de Des souris et des hommes, fait dire à l'un de ses personnages :

«Je le sais bien, tu l'as dans la main, qu'est ce que tu as dans la main que tu caches? - J'ai rien - Bien vrai, allons donne moi ça!»

En fait, ce personnage tient une souris dans la main et c'est une souris morte car il n’a pas su « maîtriser son comportement » vis-à-vis de l'animal. 
Un de nos objectifs en recherche expérimentale est précisément d'appréhender l'animal dans sa globalité et de tenter de comprendre ses comportements sans faire automatiquement référence à celui de l'homme.

Il existe des processus cellulaires intimes qui sont les mêmes quel que soit l'organisme vivant; tout de suite vient à l'esprit le code génétique. Dans ce cadre, 1953 a été une année charnière dans l'évolution de nos connaissances sur le vivant. Dans la revue Nature (25 avril 1953), J. Watson et F. Crick décrivaient pour la première fois la structure de l'ADN en double hélice. Les informations sur la transmission de l'information de cellule à cellule allaient révolutionner notre manière de réfléchir mais aussi d'appréhender de nouvelles voies thérapeutiques. La structure de l'ADN était donc élucidée et, chose remarquable, il apparaissait que le code d'éthique était universel. L'expérimentation animale a dès lors été bouleversée dans ses principes et des approches in vitro utilisant des cellules en culture ont été mises au point.

Si ce code est universel, la surprise a été de voir cette grande disparité entre le nombre de chromosome selon les espèces. Pour l'homme : 46, le chien : 78, le lapin : 44, le rat : 42 et puis maintenant on a une connaissance approfondie de la structure intime (les gènes) des chromosomes pour de nombreuses espèces animales. Ces facteurs génétiques qui règlent l'homéostasie au niveau des structures cellulaires voient leurs activités modulées par des facteurs exogènes (environnementaux)

Dans le domaine cardiovasculaire, ces facteurs exogènes, qui correspondent aux facteurs de risques, sont bien répertoriés ; citons par exemple : l'obésité, l'hypertension, les dyslipidémies (augmentation du taux de cholestérol circulant), les diabètes, qui sont des facteurs déterminants dans l'évolution des paramètres cardiovasculaires et l'initiation des pathologies. L'association de différents troubles rencontrés chez l'homme correspond à ce que l'on nomme actuellement le syndrome métabolique. Il est important de pouvoir reproduire chez l'animal ce syndrome ; un animal de choix est alors le lapin nourri avec un régime riche en cholestérol. Il présentera au bout de quelques semaines des modifications de la structure de ses vaisseaux qui signent le développement d'un processus d'athérosclérose.

Ce syndrome métabolique ne désigne que la partie visible de l'iceberg, puisque toute une partie non visible renferme une palette large de processus qui agissent en synergie : synergies entre des facteurs génétiques, des facteurs environnementaux et des styles de vie. Peut-on réellement avoir des modèles animaux qui reproduisent cet éventail d'événements que constitue le syndrome métabolique ? Nous avons vu que le lapin était un bon modèle, mais cette espèce est herbivore, alors que l'homme, dans la majorité des cas, a une alimentation à base de végétaux et de viandes (maigres et grasses). Il faut donc être très prudent dans les extrapolations des résultats obtenus dans ce cadre et le recours aux essais cliniques est indispensable.

Il est également très important de rappeler que, génétiquement, on peut se demander si le type d'alimentation qui existe actuellement chez l'homme correspond tout à fait à ce qui était programmé dans sa physiologie ! En effet, l'homme était certainement structuré pour chercher sa nourriture et utiliser les ressources qui lui étaient apportées par la nature de manière rapide : les végétaux. L'alimentation carnée devait être exceptionnelle ; elle nécessitait une activité de chasseur qui n'était certainement pas dans tous les cas couronnée par un «trophée ». À partir de là, des extrapolations ont été faites pour savoir quelle était la caractéristique biologique de l'homme préhistorique : il apparaît que son 
cholestérol plasmatique était certainement à une valeur bien plus faible que celle rencontrée aujourd'hui chez les humains. Cette valeur est d'autant plus élevée que la société à laquelle appartient l'individu est dite développée. Il existe toutefois, rappelons-le, des états génétiques caractérisés par des hypercholestérolémies dites familiales où le bilan lipidique est perturbé indépendamment des habitudes nutritionnelles. Les lapins du type «Watanabe » présentent spontanément des modifications des constantes lipidiques, ils pourront donc être retenus pour des études pharmacologiques ayant pour objectif de découvrir des substances aux propriétés normolipémiantes.

Tableau 4. Modèles d'infarctus du myocarde et de reperfusion chez des animaux présentant une dyslipidémie

\begin{tabular}{lc}
\hline Rat et autres animaux & $\begin{array}{c}\text { Ligature artère coronaire } \\
\text { et levée de la ligature }\end{array}$ \\
\cline { 2 - 2 } $\begin{array}{l}\text { Lapin } \\
\text { Cochon }\end{array}$ & Régime induisant une athérosclérose \\
\hline
\end{tabular}

Pour revenir maintenant aux modèles animaux qui se rapprochent le plus des pathologies humaines, je voudrais rappeler que le rat n'est certainement pas l'animal de choix dans l'étude de l'athérosclérose. Le lapin ou le porc, comme nous l'avons évoqué, soumis à un régime riche en cholestérol va développer au niveau vasculaire toutes les caractéristiques histologiques de l'athérosclérose. On pourra ainsi étudier, sur ces animaux rendus « malades », l'action de drogues pharmacologiques susceptibles de limiter le développement de la plaque d'athérosclérose au niveau artériel (Tableau 4). En clinique, lorsqu'une artère coronaire présente une plaque d'athérosclérose et que cette plaque non stable est le siège de l'installation d'un thrombus (caillot), il s'ensuit un processus d' « ischémie myocardique » (infarctus du myocarde). Une partie du cœur non alimentée par le sang peut se nécroser, l'atteinte est alors irréversible. Afin de protéger le myocarde de la progression de cette souffrance, ont été mises au point au cours des dernières décennies, des modalités de désobstructions. Un pas majeur dans ce domaine a été la mise à disposition du cardiologue de stents (ressorts) qui, introduits dans l'artère malade et déployés, restaurent la lumière vasculaire. Dans ce domaine de la recherche cardiovasculaire, le passage de l'expérimentation animale à l'homme a été très rapide. Les bénéfices pour les patients ont été spectaculaires, mais dans un pourcentage de cas significatif, l'artère réagit à ce « corps étranger » et tend à l'englober ; c'est le phénomène de la « resténose ». $\mathrm{Au}$ sein de notre Laboratoire, nous étudions depuis plusieurs années l'incidence de la pose d'un stent sur les processus de prolifération des cellules de la paroi vasculaire. Il est important de connaître les conditions dans lesquelles cette resténose est susceptible de se produire. Les animaux de choix dans l'étude des vaisseaux du cœur sont le chat, le chien et le porc. Toutes les espèces ne sont pas équivalentes sur le plan de la distribution des vaisseaux coronaires. Alors, qu'un animal comme le cobaye a un réseau de suppléances multiples, les cœurs du chien et du chat présentent des collatérales et des anastomoses en quantité moyenne, le porc a un réseau de suppléance modeste. Il sera donc très important de préciser les conditions expérimentales lors de chaque protocole opératoire. L'animal qui se rapprocherait le plus de l'homme sur le plan de la vascularisation coronaire est le 
porc. De plus, compte tenu de la taille de l'organe, il est envisagé dans les prochaines années des greffes cardiaques de cœur de porc à l'homme après avoir « humanisé » le greffon.

Dans le cadre des recherches cardiovasculaires, une attention tout à fait particulière a été portée à la fréquence cardiaque. On sait que le cœur humain bat une fois par seconde soit 96400 fois par jour. Existe-t-il des correspondances entre l'intensité de la fréquence cardiaque et les caractéristiques biologiques d'une espèce animale ? Comme je l'ai signalé précédemment, plus l'animal est petit, plus sa fréquence cardiaque est élevée ainsi le cœur de souris bat aux environs de 500 fois par minute alors que l'homme a une fréquence moyenne de 60 battements par minute. Il est particulièrement curieux de constater que pour chaque espèce animale, comme pour l'homme, il existerait une programmation génétique d'un nombre de fréquences cardiaques pour une vie. Ce nombre est voisin de $10^{10}$. Il convient de garder en mémoire que le myocarde possède dès la naissance son patrimoine de cellules également 10 à la puissance 10. Magie des nombres ! Le vieillissement cardiaque sera caractérisé en partie par une perte de cellules (Figure 3) et l'insuffisance cardiaque est susceptible d'apparaître.

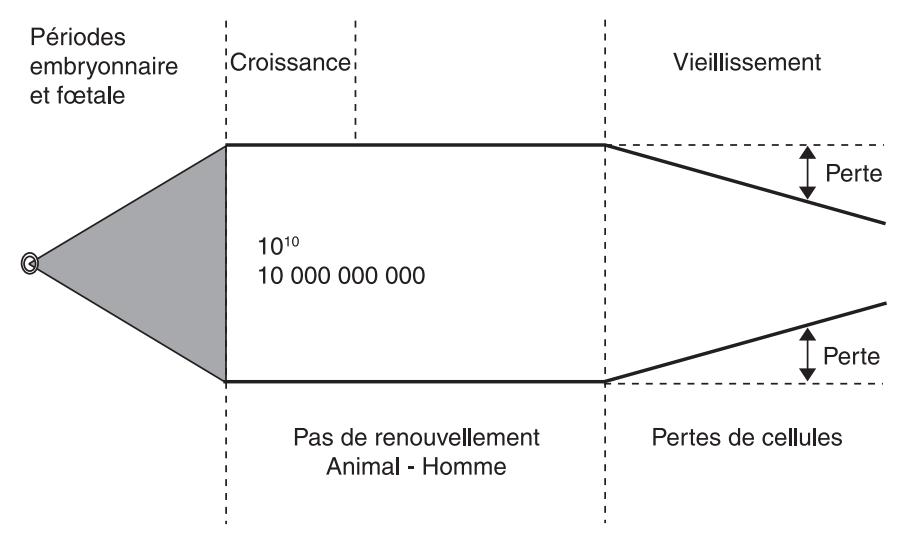

Figure 3. Nombre de cardiomyocytes dans un cœur (humain).

Dans le cadre des travaux qui sont effectués actuellement et qui permettent d'appréhender de nouvelles approches de la protection cardiaque, un volet tout à fait nouveau est apparu au cours de ces dernières années, c'est la protection induite par des cellules circulantes au niveau sanguin: ce sont les cellules progénitrices issues de la moelle osseuse (Figure 4). Des travaux expérimentaux nombreux sont en cours, ils permettent de caractériser ces cellules circulantes et de préciser les conditions dans lesquelles ces cellules vont se modifier pour devenir des cellules myocardiques, vasculaires, nerveuses. Cette toute nouvelle connaissance du renouvellement des cellules offre des perspectives majeures de la protection tissulaire. Ici encore l'expérimentation rejoint très vite les travaux cliniques. Actuellement, la thérapie génique est en plein essor, mais il existe toutefois des particularités d'espèces et des particularités pour chaque individu. Ce sont des espoirs, des attentes, des doutes et des désespoirs, mais les sciences avancent à petits pas avec leurs succès et leurs remises en cause.

Pour terminer sur ce point, je voudrais évoquer ce qu'est la genèse d'un médicament entre la première phase qui correspond au criblage de molécules jusqu'au moment où 
la substance deviendra un médicament. On estime, actuellement, que la première phase qui correspond à la période où le médicament apparaît après les procédures administratives, peut durer 10 ans. Au départ, plus de 10000 molécules sont testées et seule une des molécules verra le jour comme médicament. Après cette phase de recherche initiale, préclinique et clinique, le dossier est alors prêt pour la phase de commercialisation et de pharmacovigilance. En France, l'expiration du brevet se fait au bout de 20 ans. Des études se sont intéressées, bien entendu, au coût de l'innovation, c'est une croissance exponentielle depuis 20 ans. Si l'on considérait qu'en 1986, le coût d'une molécule innovante était de 100 millions de dollars, elle est actuellement 800 millions de dollars, donc un facteur 8 .

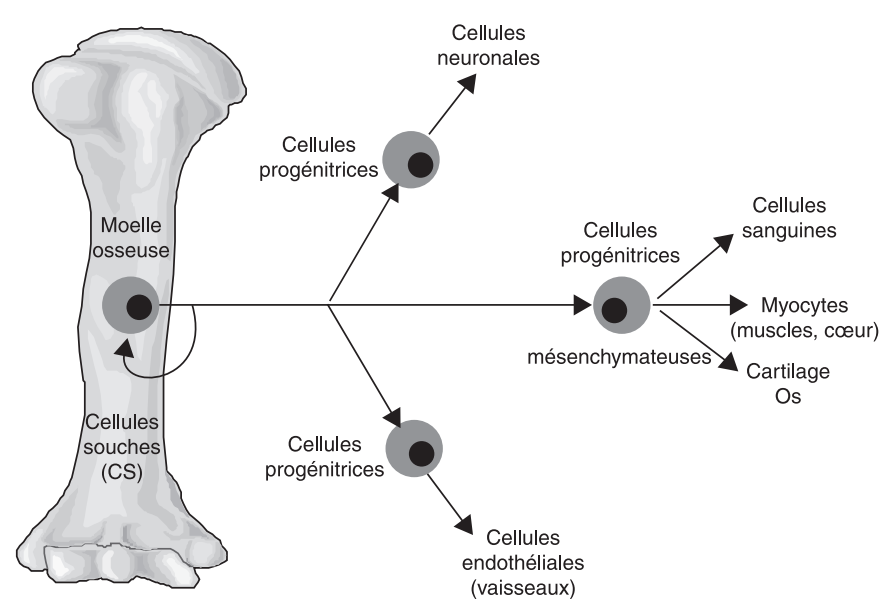

Figure 4. Cellules souches et progénitrices.

Je profiterai de cette revue pour envisager un certain nombre de questions posées par l'expérimentation animale, et tenter d'y répondre, du moins en partie.

La polémique autour de la légitimité de l'expérimentation sur les animaux a pris, on en a tous conscience, un tour passionnel durant ces dernières décennies. Ce sont développées des attitudes qui ont certainement été nécessaires pour une prise de conscience globale relative au bien-fondé de l'expérimentation animale. La question majeure a été la suivante : pouvons-nous continuer à utiliser des animaux en laboratoire pour la recherche médicale, et si oui, dans quelles conditions? Le sort que nous réservions aux animaux de laboratoire a pu paraître inévitable et même équitable à nombre de chercheurs, mais une des caractéristiques de la science n'est-elle pas de douter et de se remettre en cause ? En effet, utiliser des espèces animales au profit de la santé pouvait paraître un objectif tout à fait normal, puisque c'était en fait pour le bien de la santé humaine, sachant que pour survivre nous avons la « nécessité » de consommer du vivant végétal et animal. Le devoir et aussi le droit d'expérimenter sont aujourd'hui fortement contestés. Il existe d'ailleurs maintenant des textes, comme nous l'avons indiqué, qui régissent les conditions de l'expérimentation animale. Ce cadre législatif ne nous dispense pas de nous poser des questions personnelles et des questions en tant que directeur de laboratoire de recherche. Les droits de l'animal, que certains ont cru devoir déclarer, ne sont pas forcément tout à fait admis, en effet, sur le plan de la transposition de l'homme à l'animal. L'homme se place 
dans une situation qui est certainement ambiguë. En effet, le comportement de l'animal correspond à un mode de vie qui peut nous être complètement étranger. On ne dira jamais assez combien un fossé existe entre l'homme et l'animal, malgré des ressemblances et une continuité évolutive évidentes. Les sciences doivent s'efforcer d'appréhender la nature et les modalités spécifiques du comportement animal. Mais peuvent-elles y parvenir réellement, compte tenu du fait que, par exemple, chez le rat, la vie sociale existe mais certainement pas dans les mêmes conditions qu'en laboratoire, c'est-à-dire dans un espace clos avec la boisson et la nourriture à volonté. Notre génération a su observer les mouvements, les réactions des animaux de laboratoire, et elle a été formée en psychopharmacologie animale. Ces options ont permis le succès des découvertes concernant les psychotropes, bien plus tard, et seulement partiellement, la connaissance des mécanismes neuronaux impliqués dans les actions de ces composés. On voit que, malgré les avancées du savoir, bien des problèmes rencontrés restent sans réponse, dans le domaine scientifique stricto sensu, mais aussi celui de l'éthique.

Une autre question rencontrée par l'expérimentateur est la suivante : les animaux ontils des émotions ? La réponse est oui. Existe-t-il dès lors une analogie avec ce que l'homme considère comme une émotion? À cette nouvelle question, les réponses sont multiples. Certaines sont d'ordre physiologique. Il est ainsi possible de doser au niveau sanguin des substances endogènes, marqueurs biologiques du niveau de stress : par exemple, l'hormone surrénalienne l'adrénaline qui est qualifiée hormone du stress. Un niveau de stress peut être appréhendé par la variation de ce paramètre. D'autres réponses sont d'ordre comportemental. Elles n'impliquent pas forcément un stress, mais doivent être appréhendées en termes de transfert entre l'homme et l'animal. Il est bien connu qu'un animal se conditionne à l'environnement qui lui est imposé. Ainsi, lorsque chaque jour vous le prenez pour le changer de cage ou le mettre dans un appareil qui quantifie son activité exploratrice, il devient peu à peu très docile. Ce conditionnement est très rapide et il signe des processus centraux d'apprentissage. Si un animal ne présente pas ce type d'adaptation et apparaît peu « coopérant » peut-on alors envisager, chez cet animal, un niveau de souffrance ? À quel niveau de souffrance correspond une modification d'un comportement? Cette modification comportementale peut dépendre de l'animal, mais peut dépendre également de l'environnement. Il est bien connu, par exemple, qu'il ne faut pas faire séjourner dans la même pièce, des cages de rats et de souris, il y a « incompatibilité » qui peut être qualifiée de « génétique ». On peut donc penser que les territoires sont distincts.

En tant que chercheurs, nous avons tous été sensibilisés au comportement de l'animal et à sa réaction face à notre présence. Il existe toutefois de nombreuses difficultés pour approcher avec certitude ces modifications comportementales. La première qui me vient à l'esprit correspond par exemple, à une modification de la faim et la soif chez l'animal. La prise de nourriture est un index particulièrement validant et validé. Dans de nombreux cas, ces modifications comportementales ne peuvent être quantifiées que dans le cadre d'un groupe d'animaux. Ainsi, quand la nourriture est en quantité insuffisante, une relation nouvelle va se créer au sein du groupe pour des survies individuelles. Les plus forts l'emporteront sur les plus faibles. Une situation tout à fait particulière est rencontrée chez la femelle qui protège ses petits. Cette situation a été très étudiée et les recherches mettent bien en évidence l'influence des conditions de vie en laboratoire sur le comportement des animaux, dont il est bien difficile d'identifier les dimensions exclusivement naturelles propres à l'espèce. 
Un des objectifs de l'évolution actuelle de l'expérimentation animale est de mieux cerner les conditions du bien-être de l'animal. Il est certain que les mesures comportementales sont les plus adaptées pour juger de ce bien-être. Au final, il n'en demeure pas moins que c'est notre perception humaine de son comportement qui va être décisive.

La responsabilité du chercheur est donc importante et particulièrement difficile à assumer. On lui demande en effet d'évaluer à la fois la nécessité et la pertinence du protocole expérimental qu'il a établi et d'appréhender " objectivement » le niveau de douleur qui peut être occasionné par cette expérimentation. Des articles du code rural donnent un certain nombre d'indications pour supprimer ou tout au moins limiter la douleur. Ces textes sont les suivants : " les expériences sur des animaux vivants qui peuvent entraîner des souffrances doivent être pratiquées sous anesthésie générale ou locale ou après recours à des procédés analgésiques équivalents sauf, si la pratique de l'anesthésie ou de l'analgésie est considérée comme plus traumatisante pour les animaux que l'expérience elle-même ». ${ }^{1}$ Des expériences sans anesthésie ou analgésie, lorsqu'elles sont susceptibles d'entraîner des douleurs intenses chez l'animal, doivent être expressément déclarées et justifiées auprès des autorités de tutelle, ce qui parait tout à fait normal. Les obligations de supprimer et de limiter au maximum la douleur, doivent toujours être prises en compte. Si un animal est gardé en vie, il doit recevoir les soins nécessaires pour son état de santé et être placé sous la surveillance d'un vétérinaire ou d'une autre personne compétente titulaire d'une autorisation d'expérimenter et responsable du protocole.

Dans cette approche de la responsabilité du chercheur, il devient important que ce dernier ait une connaissance approfondie des procédés qui sont susceptibles de prévenir le développement de la douleur. Il existe des protocoles spécifiques pour chaque anesthésie, qu'elle soit locale ou générale. Il existe donc dans le domaine l'expérimentation animale à des fins de recherche, des protocoles qui sont des protocoles codifiés et qui doivent être validés par les comités mis en place dans chaque structure de recherche comme nous l'avons déjà précisé.

Les autorités de tutelle ont codifié un contrôle de l'application de la réglementation qui me paraît tout à fait légitime. Ce contrôle s'établit à tous les niveaux et les sanctions peuvent être pénales. Dans un décret paru en mars 2005, il a été créé un Comité national de réflexion éthique sur l'expérimentation animale. Ce comité émet des avis sur des questions éthiques soulevées par cette expérimentation, élabore une charte nationale et fait des recommandations des méthodes pour améliorer le bien être des animaux. Une des réflexions majeures qui est également intervenue au cours de ces dernières décennies, c'est d'envisager les méthodes alternatives à l'expérimentation animale. Ces méthodes alternatives ont fait l'objet de très nombreux écrits. Elles ont pour but de réduire le nombre des animaux utilisés en recherche médicale ou scientifique. Elles permettent également de diminuer la souffrance potentielle des animaux qui seront utilisés dans ces protocoles. Peut-on pour autant remplacer totalement l'expérimentation animale telle qu'elle a été envisagée au cours de ces dernières années? Le remplacement peut-il être total ou partiel ? L'apport d'un certain nombre de nouvelles techniques doit nous aider, peut être, à une nouvelle réflexion sur les modalités qui permettraient de préciser la toxicité potentielle d'un composé au niveau de l'organisme entier. On peut avoir recours à des systèmes in vitro, des systèmes sur cellules isolées ou tissus isolés, sur des organismes

\footnotetext{
${ }^{1}$ Décret 87-88 du 19 octobre 1987, chapitre 1.
} 
inférieurs ou tout au moins qualifiés inférieurs comme des invertébrés ou sur des microorganismes. Une possibilité, qu'il ne faut pas négliger, d'une information majeure sur l'efficacité, la toxicité potentielle de certains composés passe par la bio-informatique et les techniques physico-chimiques. On peut penser que dans des systèmes de modélisation par informatique, à l'aide de logiciels spécifiques, on est susceptible d'avoir une certaine approche de l'interaction entre les molécules testées et des structures protéiques qui participent à l'homéostasie cellulaire. De très nombreuses recherches sont actuellement en cours afin de mieux connaître cette ouverture.

Il faut bien remarquer que ces approches nouvelles demandent une amélioration des technologies et doivent être ciblées sur des tissus spécifiques qui seront par exemple le cartilage, les cardiomyocytes (cellules contractiles du cœur), les cellules musculaires lisses ou des cellules qui ont des potentialités de se renouveler rapidement. Dans le domaine cellulaire, il est possible également d'envisager que les études soient ciblées sur des fractions sub-cellulaires au niveau d'organiques cellulaires qui sont impliqués dans les phénomènes de détoxification. Dans ce cadre, beaucoup de travaux ont été réalisés, notamment afin de développer des modèles par la mise au point de cultures cellulaires animales et dans certains cas, de cellules humaines. On a tous en mémoire que dans le domaine de l'évaluation de la tolérance locale, cutanée et oculaire, des avancées importantes ont été obtenues.

Il paraît capital à ce stade de bien discerner les études qui sont du domaine réglementaire, des études qui ont pour objectif de mieux appréhender les mécanismes cellulaires qui sous-tendent une toxicité. C'est le passage du screening à la recherche plus large. Un des avantages du test in vitro est sa rapidité. Les tests de toxicité anormale réalisés sur chaque produit fini dans le milieu industriel visent à déceler une toxicité quelle qu'en soit la nature, une erreur dans les conditions de fabrication conduisant à une contamination bactérienne, virale ou fongique possible. Dans ce cadre, des tests in vitro peuvent être pratiqués rapidement et remplacent l'animal. En revanche, il faut bien évoquer les limites de ces tests et ne pas perdre de vue qu'ils sont susceptibles de passer à côté de contaminations qui ne peuvent pas être révélées simplement sur des tests simples in vitro. Toutefois, c'est un avantage, mais comme tout avantage, il présente des limites.

On retrouve très souvent dans les articles relatifs à l'expérimentation par substitution, la règle des trois R. - Remplacement par les méthodes in vitro, - Réduction du nombre d'animaux, - Raffinement de la méthodologie pour réduire la douleur et la détresse animale. Depuis une vingtaine d'années, le nombre global d'animaux de laboratoire utilisés dans le milieu industriel ou académique, diminue régulièrement. Cette diminution dépasse $50 \%$ dans beaucoup de cas ; ce qui constitue un excellent résultat, étant donné la croissance du marché et le nombre de molécules testées. Cependant, je tiens de nouveau à tempérer ce genre d'enthousiasme. En effet, ces tests in vitro ne correspondent qu'à un volet d'une protection qui doit, dans certains cas, être étendue à l'expérimentation in vivo. Passer d'un modèle in vitro ou par «modélisation » à un modèle in vivo doit se faire après réflexion et connaissance préalable de l'objectif recherché. Le critère du bon modèle est également à prendre en compte. Ce modèle doit être reproductible. Il doit permettre de travailler dans des conditions de sécurité fiable et si possible pas trop contraignantes. Il doit se développer au sein d'une espèce animale facile à élever et à reproduire. Ces critères de bon modèle sont valables pour chaque chapitre de pharmacologie. 


\section{Conclusion}

Pour terminer, je crois qu'il est important de rappeler deux principes de la démarche scientifique : premièrement, il faut se méfier de la routine, deuxièmement, il y a toujours des améliorations possibles à apporter. On ne peut oublier qu'au cours de ces vingt dernières années on a vu la mise en œuvre, grâce à l'expérimentation animale, de traitements efficaces et bien tolérés dans l'hypertension, l'insuffisance cardiaque, l'athérosclérose et dans de nombreuses autres pathologies. Ce volet positif ne doit pas être occulté, car il nous concerne tous et surtout les générations à venir. Il restera toujours à découvrir de nouvelles approches thérapeutiques, certainement plus ciblées sur l'individu en particulier. La spécificité génétique individuelle est maintenant mieux appréhendée, mais il importe de préserver l'intégrité et la liberté de chaque homme dans la conduite de sa vie. La question se pose-t-elle dans les mêmes termes en ce qui concerne l'animal ? Doit-on, pour des raisons éthiques, refuser toute instrumentalisation de celui-ci à des fins qui servent exclusivement les intérêts humains ? Le problème des valeurs paraît de plus en plus crucial à l'heure où les recherches qui tendent à montrer la proximité de nature et la continuité entre l'homme et l'animal sont nombreuses et convergentes. Si nos « frères inférieurs » sont bien des frères, peut-on accepter de les traiter selon des valeurs et des normes qui ne sont pas celles de la fraternité humaine ? En tant que chercheur, je n'ai évidemment pas la réponse, même si mon domaine d'expertise m'incite à un certain scepticisme à l'égard des thèses qui rapprochent trop radicalement et péremptoirement l'animal de l'homme. En tant que personne morale, je ne peux cependant m'empêcher de me les poser, et ma perplexité est grande. 


\section{Bibliographie}

Adolphs R., 2003, Cognitive neuroscience of human social behaviour, Nature Reviews Neuroscience, 4, pp. 165-178.

Agamben G., 2002, L'ouvert, de l'homme et de l'animal, trad. franç., Paris, Payot et Rivages. Alexander R.D., 1987, The Biology of Moral Systems, New York, Aldine de Gruyter.

Amselek V. P., 1997, La part de la science dans les activités des juristes, D. 1997, Chron.

Ancet P., 2006, Phénoménologie des corps monstrueux, Paris, Puf.

Andersson M., 1994, Sexual Selection, Princeton, Princeton University Press.

Anonyme, Miracle arrivé dans la ville de Genève en cette année 1609 d'une femme qui a faict un veau à cause du mespris de la puissance de Dieu et de Madame Saincte Marguerite, Angoulême, rééd. Frugier, 1858.

Antoine S. et Nouët J.-C., 2003, La Fondation Ligue Française des Droits de l'Animal, éd. LFDA. Antoine S., 1996, Le droit de l'animal, évolutions et perspectives, D. 1996, Chron.

Aristote, 2001, Éthique à Nicomaque, trad. franç., Paris, Vrin.

Aristote, 1961, De la Génération des Animaux, IV 3, 769, trad. franç., Paris, Les Belles Lettres.

Aristote 1983 , La Politique, trad. franç., Paris, Nathan.

Atias C., 2002, Épistémologie juridique, Dalloz, collection Précis Droit privé, $1^{\text {re }}$ éd., p. 89.

Bentham J., 1970, An Introduction to the Principles of Morals and Legislation, in : J.-H. Burns et H. L. A. Hart (eds.), The collected works of Jeremy Bentham, London, University of London, The Athlone Press, p. 12.

Benveniste E., 1966, Problèmes de linguistique générale, trad. franç., Paris, Gallimard. 
Berdiaeff N., 1935, De la destination de l'homme. Essai d'éthique paradoxale. Paris, Éditions Je sers.

Bergel J.-L, Brushi M. et Cimamonti S., 2000, Les biens, Traité de droit civil, sous la direction de J. Ghestin, LGDJ, 453.

Bergson H., 1970, Les Deux sources de la morale et de la religion, Euvres, Paris, Puf.

Bertin L., 1950, Regards sur la nature et ses mystères. La terre, les bêtes, les hommes et les monstres, Paris, éditions du Pavillon.

Blanckaert C., 1991, Premier des singes ou dernier des hommes, Alliages, 7/8, Nice, Anais et z'éditions.

Bogdan R., 1988, Freak-show. Presenting human oddities for amusement and profit, Chicago, The University of Chicago Press.

Bonnardel Y., 2005, En finir avec l'idée de Nature, renouer avec l'éthique et la politique, Les Temps Modernes, 630, pp. 466-490.

Braggio J.T., Hall A.D., Buchanan J.P. et Nadler R.D., 1979, Cognitive capacities of juvenile chimpanzees on a Piagetian-type multiple-classification task, Psychological Reports, 44, p. 1097.

Bremmer G., 1980, "The infant's understanding of space", in: M. Cox (ed.), Are young children egocentric?, London, Concord, pp. 281-309.

Bressou C., 1944, Les Monstres, de la légende à la réalité, Alençon, imprimerie Poulet Malassis.

Bromberger C., Morel A., 2005, Limites floues et frontières vives. Des variations culturelles en France et en Europe, Paris, CTHS.

Brunois A., 1984, L'animal, sujet de droit, colloque «Droit de l'animal et pensée contemporaine », 15 octobre 1984, LFDA.

Buffon G.-L.L. 1853-1855, Discours sur la nature des animaux (1753), Histoire naturelle, t. II, t. IV, dans l'éd. Flourens, Paris, Garnier Frères.

Buffon G.-L. L., 1955, La nomenclature des singes (1766), Histoire naturelle, t. IV dans l'éd. Flourens, Paris, Garnier Frères.

Burgat. F., 1997, Animal, mon prochain, Paris, Odile Jacob.

Buss D.M., 2003, The Evolution of Desire. Strategies of Human Mating, New York, édition révisée Basic Books.

Buytendijk F. J.-J., 1965, L'Homme et l'animal. Essai de psychologie comparée, [1958], trad. franç., Paris, Gallimard.

Buytendijk F.J.J., 1952, Dans son Traité de psychologie animale [1952], trad. franç., Paris, Puf.

Buytendijk F.J.J., 1965, L'Homme et l'animal, trad. franç., Paris, Gallimard.

Canguilhem G., 1970, Études d'histoire et de philosophie des sciences, Paris, Vrin.

Canguilhem G., 1971, La connaissance de la vie, La Monstruosité et le monstrueux, Paris, Vrin. 
Carbonnier J., 2001, Flexible droit. Pour une sociologie du droit sans rigueur, Paris, LGDJ, $10^{\mathrm{e}}$ éd.

Carbonnier J., 2004, Droit civil, vol. 2, Les biens, Les obligations, Paris, Puf, « Quadrige Manuels ».

Carneiro Alves A., 1987, La Tératologie dans l'art : les monstres biologiques ont-ils inspiré les artistes?, Thèse de médecine, Nice.

Cartwight J., 2000, Evolution and Human Behaviour, Londres, MacMillan.

Castignone S., 1988, Concept de personne et droits des animaux, in : Homme-Animal-Société, t. II, Droit et animal, Toulouse, Presses de l'Institut d'Études politiques de Toulouse.

Castoriadis C., 1975, L'institution imaginaire de la société, Paris, Seuil.

Cavalieri P. et Singer P. (ed.), 1994, The Great Ape Project. Equality beyond Humanity, New York, Saint-Martin's Press.

Cavalieri P., 1991, Quanto cantano gli animali ?, Milano, Animus/G. Mercandalli ed.

Cavalieri P., 2000a, Les droits de l'homme pour les grands singes non humains ?, Le Débat 108, janv.-fév.

Cavalieri P., 2000b, L'humanité au-delà des humains, Le Débat, 108, janvier-février.

Cavalieri P., 2000c, Humanité et égalité, Le Débat, 109, mars-avril.

Cavalieri P., 2004, Vers une super-humanité totale, Science et Avenir, hors série, Les animaux ont-ils un sens moral ?, juin-juillet.

Ceard J., 1996, La Nature et les prodiges. L'insolite au XVI siècle (1977), Paris, Droz.

Cézilly F., 2006, Le Paradoxe de l'Hippocampe. Une Histoire Naturelle de la Monogamie, Paris, Buchet-Chastel.

Cézilly F., Giraldeau L.-A. et Théraulaz G., 2006, Les Sociétés Animales. Lions, Fourmis et Ouistitis, Paris, Le Collège de la Cité, Le Pommier.

Chapoutier G., 1992, Les droits de l'animal, Paris, Puf, « Que sais-je »?

Cheney D.L. et Seyfarth R.M., 1990, How monkeys see the world, Chicago, University of Chicago Press.

Collier-Baker E., et Suddendorf T., 2006, Do chimpanzees (Pan troglodytes) and 2-year-old children (Homo sapiens) understand double invisible displacement?, Journal of Comparative Psychology, 120, pp. 89-97.

Comte-Sponville A., 1995, Sur les droits des animaux, Esprit, décembre.

Condillac E., 2004, Traité des animaux, éd. M. Malherbe, Paris, Vrin.

Crespi F., 1983, Médiation symbolique et société, Paris, Librairie des Méridiens.

Daigueperse C., 1981, L'animal, sujet de droit, réalité de demain, Gaz. Pal. 1981 (1 $\left.{ }^{\mathrm{er}} \mathrm{sem}.\right)$, doc. p. 160.

Dalla Bernardina S., 1988, Hédonistes et ascètes. Latins et Septentrionaux à la chasse au chamois dans les Alpes italiennes, Le Monde alpin et rhodanien (La haute montagne. Vision et représentations). 
Danchin E. et Cézilly F., 2005, La sélection sexuelle : un autre processus évolutif, in : Écologie Comportementale (E. Danchin, L.-A. Giraldeau et F. Cézilly eds.), Paris, Dunod.

Danti-Juan M., 1996, La contribution du nouveau Code pénal au débat sur la nature juridique de l'animal, Rev. dr. rur. 248, déc.

Darwin C., 1859, On the Origin of the Species by Means of Sexual Selection, or Preservation of Favoured Races in the Struggle for Life, Londres, Murray.

Darwin C., 1871, The Descent of Man and Selection in Relation to Sex, Londres, Murray.

Darwin C., 1981, L'Expression des émotions chez l'homme et les animaux, trad. franç., Paris, éd. Complexe.

Darwin C., 1999, La filiation de l'homme, trad. franç., Paris, éd. Syllepse.

Daubenton L. J.-M., Sur les différences de situation du grand trou occipital dans l'homme et dans les animaux, Mémoires de l'Académie royale des sciences, p 568-575.

Dawkins R., 1978, Le gène égoïste, Paris, Menges.

De Waal F, 1996, Good Natured. The Origins of Right and Wrong in : Humans and Other Animals, Cambridge, Mass : Harvard University Press (trad. franç., 1997, Le bon singe : les bases naturelles de la morale, Paris, Bayard).

De Waal F., 2002, De la réconciliation chez les primates, Paris, Flammarion.

De Waal F., 2000, Le singe en nous, Paris, Fayard.

De Waal F., Chimpanzees Politics, London, Jonathan Cape, 1982 (trad. franç. La politique du chimpanzé, Monaco, éd. du Rocher, 1991).

De Waal F., Entretien avec Sophie Laurent, La Croix, 21 Juin 1997.

De Waal F.B.M., Dindo M., Freeman C.A. et Hall M.J., 2005, The monkey in the mirror : Hardly a stranger, Proceedings of the National Academy of Sciences, 102, pp. 1140-1147.

Dejours C., 1993, Travail, usure mentale, Paris, Bayard.

Dejours C., 1998, Souffrance en France, Paris, Seuil.

Demogue R., 1909, La notion de sujet de droit, caractères et conséquences, RTD civ.

Dennett D. C., 2000, Darwin est-il dangereux? trad. franç., Paris, Odile Jacob.

Descartes R., 1964-1974, Euvres, t. 9, Paris, éditions Adam-Tannery (AT), CNRS.

Descola P., 2004, Le sauvage et le domestique, Communications, 76.

Descola P., 2005, Par-delà nature et culture, Paris, Gallimard.

Desmoulin S., 2006a, Protection des animaux et condition juridique de l'animal en droit français, Revue interdisciplinaire d'études juridiques, 2006-57, pp. 37-75.

Desmoulin S., 2006b, L'animal, entre Science et Droit, Presses universitaires d'Aix-Marseille.

Despret V., Porcher J., 2007, Etre bête, Arles, Actes Sud.

Diamond A., 1991, "Neuropsychological insights into the meaning of object concept development”, in : S. Carey, et R. Gelman (eds.), The epigenesis of mind, Hillsdale, Lawrence Erlbaum, pp. 67-110. 
Diderot D., 1994, Éléments de Physiologie, Euvres, t. I (Philosophie), Paris, R. Laffont.

Diderot D., 1994, Le rêve de d'Alembert, Euvres, t. I (Philosophie), Paris, R. Laffont.

Diderot D., 1994, Pensées sur l'interprétation de la nature, Euvres, t. I (Philosophie), Paris, R. Laffont.

Diderot D., 1994, Suite de l'entretien entre d'Alembert et Diderot, Euvres, t. I (Philosophie), Paris, R. Laffont.

Doré F. Y., 1990, Search behavior of cats (Felis catus) in an invisible displacement test: Cognition and experience, Canadian Journal of Psychology, 44, pp. 359-370.

Doré F.Y. et Dumas C., 1987, Psychology of animal cognition : Piagetian studies, Psychological Bulletin, 102, pp. 219-233.

Dosner-Dolivet A., A. Scemama A., 1983, Note sous Paris, 11 janv., Gaz. Pal., 2, p. 412.

Dupanloup I., Pereira L., Bertorelle G., Calafell F., Prata M.J., Amorim A. et Barbujani G., 2003, A recent shift from polygyny to monogamy in humans is suggested by the analysis of worldwide Y-chromosome diversity, Journal of Molecular Evolution, 57, pp. 85-97.

Durand G., 1992, Les Structures anthropologiques de l'imaginaire, Paris, Dunod.

Dutour O., Hublin J.P. et Vandermeersch B., 2005, Origines et évolution des populations humaines, Paris, CTHS.

Eddy T.J., Gallup G.G. et Povinelli D.J., 1996, Age differences in the ability of chimpanzees to distinguish mirror-images of self from video images of others, Journal of Comparative Psychology, 110, pp. 38-44.

Eldredge N., 2004, Why We Do It. Rethinking Sex and the Selfish Gene, New York, Norton et Co.

Engelhardt E., 1900, De l'animalité et de son droit, Paris, éd. A. Chevallier-Marescq et Cie.

Fabre D., 2005, Limites non frontières du sauvage, L'Homme, 175-176, Vérités de la fiction.

Farjat G., 2002, Entre les personnes et les choses, les centres d'intérêts (Prolégomènes pour une recherche), RTD civ., (avril-juin), Art., p. 221.

Fédération nationale des éleveurs de porcs, Compte rendu de l'Assemblée générale de mars 2006 .

Ferry L. et Germé Cl., 1994, Des animaux et des hommes. Anthologie des textes remarquables écrits sur le sujet, $d u X v^{e}$ siècle à nos jours, Paris, Librairie générale française, Le livre de poche.

Ferry L., 1992, Le nouvel ordre écologique (L'arbre, l'animal et l'homme), Paris, Grasset.

Fichte J.G., 1984, Fondement du droit naturel selon les principes de la doctrine de la science, trad. franç., Paris, Puf, « Quadrige ».

Fisher H., 1992, The Anatomy of Love, New York, Fawcett Books.

Flavell J.H., Flavell E.R. et Green F.L., 1983, Development of the appearance-reality distinction, Cognitive Psychology, 15, pp. 95-120. 
Flinn M.V., Geray D.C. et Ward C.V., 2005, Ecological dominance, social competition, and coalitionary arms races: Why humans evolved extraordinary intelligence, Evolution and Human Behavior, 26, pp. 10-46.

Fontaine A.M., 1992, L'enfant et son image. Paris, Nathan.

Fontenay E. de, 2000, Pourquoi les animaux n'auraient-ils pas droit à un droit des animaux ?, Le Débat, 109, mars-avril, p. 138.

Foucault M., 1975, Surveiller et punir. Naissance de la prison, Paris, Gallimard.

Foucault M., 1976, Histoire de la sexualité I. La volonté de savoir. Paris, Gallimard.

Franck D., 1986, Heidegger et le problème de l'espace, Paris, Minuit.

Fricaud R.-P., 1933, Les Malades et les monstres dans la sculpture médiévale, Bordeaux, Imprimerie de l'Université.

Frost P., 2006, European hair and eye color. A case of frequency-dependent sexual selection?, Evolution and Human Behavior, 27, pp. 85-103.

Gagnon S. et Doré F. Y., 1993, Search behavior of dog (Canis familiaris) in invisible displacement problems, Animal Learning and Behavior, 21, pp. 246-254.

Gallup G.G., 1970, Chimpanzees : Self-recognition, Science, 167, pp. 86-87.

Garcha H.S. et Ettlinger G., 1979, Objects sorting by chimpanzees and monkeys, Cortex, 15, pp. 213-224.

Garrott R., White P., Vanderbidt White C., 1993, Overabundance : an issue for conservation biologists ?, Conservation biology, 7.

Geoffroy Saint-Hilaire E., 1824, Sur une nouvelle Monstruosité, nommée anencéphale de Patare, Journal Universel des Sciences Médicales, t. 36, pp. 3-4.

Geoffroy Saint-Hilaire E., 1825, Considérations générales sur la monstruosité et description d'un genre nouveau observé dans l'espèce humaine et nommé Aspalasome, Journal Complémentaire du Dictionnaire des Sciences Médicales, t. 21, 83eahier, mai 1825, pp. 4-5.

Geoffroy Saint-Hilaire E., 1826, Sciences-Antiquités, (communication à l'Académie royale des sciences, séance du 9 janvier 1826), Extrait du Moniteur, 15 janvier 1826, pp. 3-4.

Geoffroy Saint-Hilaire I., 1832-1836, l'Histoire générale et particulière des anomalies de l'organisation chez l'Homme et les animaux ou Traité de tératologie, Paris, Baillière, (3 vol.).

Giberne P., 1931, La protection juridique des animaux, thèse, Paris.

Goffi J.-Y., 1994, Le philosophe et ses animaux. Du statut éthique de l'animal, Nîmes, Jacqueline Chambon, « Rayon philo. ».

Goffi J.-Y., 2004, Qu'est-ce que l'animalité ?, Paris, Vrin.

Gouyon P.-H., 2001, Les harmonies de la Nature à l'épreuve de la biologie, Paris, Inra éditions, « Sciences en question».

Gouyon P.-H., Henry J.-P. et Arnould J., 1997, Les avatars du gène. La théorie néodarwinienne de l'évolution, Paris, Belin. 
Guichet J.-L., 2004, L'animal dans la peinture de Chardin, Dix-huitième siècle (Société française d'étude du XVIII ${ }^{\mathrm{e}}$ siècle), 36, Paris, Puf.

Guillebaud J.-C., 2001, Le principe d'humanité, Paris, Seuil.

Hamilton W., 1964, The genetical evolution of social behaviour, Journal of theoretical biology, 7.

Hardouin-Fugier E., 2002, La protection législative de l'animal (un recyclage français de la propagande nazie), Écologie et politique (Science, culture, société), 24, janv.

Haudricourt A. G., 1962, Domestication des animaux, culture des plantes et traitement d'autrui, dans La technologie science humaine, 1987, Paris, éditions de la MSH, pp. 37-46.

Hauser M. D., 2002, À quoi pensent les animaux ?, trad. franç., Paris, Odile Jacob.

Hauser M.C., Kralik J., Botto-Mahan C., Garrett M. et Oser J., 1995, Self-recognition in primates: Phylogenetic and the salience of species-typical features, Proceedings of the National Academy of Sciences, 92, pp. 10811-10814.

Heidegger M., 1992, Les concepts fondamentaux de la métaphysique, monde-finitude-solitude, trad. franç., Paris, Gallimard.

Henry M, 1985, Généalogie de la psychanalyse, Paris, Puf.

Henry M., 1987, La barbarie, Paris, Puf, « Quadrige ».

Hermitte M.-A., 2004, L'animal, sujet de droit?, in : F. Burgat, L'animal dans nos sociétés, Paris, La Documentation française, « Problèmes politiques et sociaux », 896, janv.

Heyes C. M., 1998, Theory of Mind in Nonhuman Primates, Brain and Behavioural Science, 21.

Heyes C.M., 1998, Theory of mind in nonhuman primates, Behavioural and Brain Sciences, 21, pp. 101-148.

Hollard V. et Delius J., 1982, Rotational invariance in visual pattern recognition by pigeons and humans, Science, 218, pp. 804-806.

Husserl E, 1969, Méditations cartésiennes, trad. franç., Paris, Vrin.

Iacub M., 2005, Sont-ils des choses ou des personnes? in : M. Iacub, Bêtes et victimes (et autres chroniques de "Libération”), Paris, Stock, « Les essais ».

Illes J., 2006, Neuroethics. Defining the issues in theory, pratice and policy, Oxford, Oxford University Press.

Jacob F., 1992, La Logique du vivant, Paris, Gallimard, « Tel ».

Jaisson P., 1993, La fourmi et le sociobiologiste, Paris, Odile Jacob.

Jeannerey G., 1923, La Tératologie dans l'art, La Chronique médicale, mai 1923, 5, pp. 131-138.

Jeannerod M., 2005, Le cerveau intime, Paris, Odile Jacob.

Kant E., 1968, Critique de la faculté de juger, trad. franç., Paris, Vrin.

Karmiloff-Smith A., 1992, Beyond modularity : a developmental perspective on cognitive science, Cambridge, Mass., MIT Press. 
Kitcher P., 1993, The Evolution of Human Altruism, Journal of Philosophy, vol. 90.

Kreiss N., 2005, Gestion d'une population de Marmottes alpines pour la préservation d'un paysage remarquable dans le Parc National des Écrins : expérimentation d'une méthode de contraception, Mémoire présenté en vue de l'obtention du diplôme d'études spécialisées en gestion des ressources animales et végétales en milieux tropicaux.

Kreutzer M. et Vauclair J., 2004, La cognition animale au carrefour de l'éthologie et de la psychologie, in : Vauclair J. et Kreutzer M. (éds.), L'Éthologie cognitive, Paris, éditions de la MSH, pp. 1-20.

Kropotkine P. A., 1979, L'éthique, Paris, Stock.

La Mettrie J.O. de, 2004, L'homme machine, Euvres philosophiques, éd. J.-P. Jackson, Checy, Coda.

Labbée X., 2002, La personne, l'âme et le corps, Petites Affiches, 243, 05 décembre.

L'Ane, avril 1995, 60, pp. 25-28.

Langer J., 1986, The origins of logic: One to two years. New York, Academic Press.

Larrère R., 1997, Diversité biologique et gestion des parcs et des réserves, Sciences sociales et espaces protégés.

Larrère R., 2005, communication à la journée Regards sur la nature du Groupe interdisciplinaire d'éthique de la recherche (Giere), Grenoble, MSH, 7 décembre 2005.

Lascoumes P., 1994, L'éco-pouvoir. Environnements et politiques. Paris, La Découverte.

Latour B., 1989, La science en action, Paris, Gallimard.

Le Douarin N., 2000, Des chimères, des clones et des gènes, Paris, Odile Jacob.

Leibniz G.W., 1972, Principe de la nature et de la grâce, 14, Euvres, trad. franç., Paris, Aubier-Montaigne.

Leopold, A., 1933, The Conservation Ethic, Journal of Forestry, 31.

Leopold, A., 1949, A Sand County Almanach, Oxford, Oxford University Press.

Leroi-Gourhan A., 1965, Le Geste et la parole, t. 2, Paris, Albin Michel.

Lestel D., 1996, L'animalité. Essai sur le statut de l’humain, Paris, Hatier.

Lestel D., Grundmann E., 1999, Tools, Techniques and Animals : the role of mediations of actions in the dynamic of social behaviour, Social Science Information, 38, 3.

Lestel, D., 2001, Les origines animales de la culture, Paris, Flammarion.

Levy-Bruhl V., 1992, La protection de la faune sauvage en droit français, thèse de doctorat, Lyon III.

Lin A.C., Bard K.A., Anderson J.R., 1992, “Development of self-recognition in chimpanzees (Pan troglodytes)", Journal of Comparative Psychology, 106, pp. 120-127.

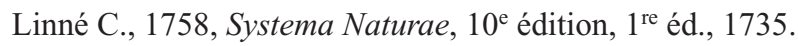

Locke J., 1972, Essai concernant l'entendement humain, trad. franç., Paris, Vrin. 
Locke J., 1983, Essai philosophique concernant l'entendement humain, III, 6, 27, et III, 6, 22, trad. franç., Paris, réimpression, Vrin.

Lombois C, 1992, Préface, in : J.-P. Marguénaud, L'animal en droit privé, Paris, Puf, p. II.

Lorenz K., 1969, L'Agression. Une histoire naturelle du mal, trad. franç., Paris, Flammarion, Champs.

Lorenz K., 1974, Trois essais sur le comportement humain et animal, trad. franç., Paris, Le Seuil, Points.

Lorenz K., 1975, L'Envers du miroir, trad. franç., Paris, Flammarion.

Lorenz K., 1984, Les fondements de l'éthologie, trad. franç., Paris, Flammarion.

Lovejoy C.O., 1981, The origins of man, Science, 211, pp. 341-350.

MacDonald K.B., 1990, Mechanisms of sexual egalitarianism in Western Europe, Ethology and Sociobiology, 11, pp. 195-238.

Machiavel N., 1952, Le Prince, ch. XVII, Oeuvres complètes, trad. franç., Paris, Gallimard, « La Pléiade ».

Maillet B. de, 1984, Telliamed, Corpus, Paris, Fayard.

Marguénaud J.-P., 1992, L'animal en droit privé, Paris, Puf.

Marguénaud J.-P., 1998, La personnalité juridique des animaux, D., Chron.

Marguenaud J-P., 1995 ; L'animal dans le nouveau code pénal, D., Chron. 187.

Marler P., The instinct to learn in : S. Carey et R. Gelman (dir.), The Epigenesis of mind: Essays in Biology and Cognition, (N. J.), Lawrence Erlbaum.

Martin E., 2002, Histoire des monstres depuis l'Antiquité jusqu'à nos jours, Paris, Reinwald, (1880), réed, Grenoble, Jérôme Millon.

Marx K., Engels F., 1975, L'idéologie allemande, trad. franç., Paris, Éditions sociales.

Masserman J H., Wechkin S., Terris W., 1964, Altruistic behaviour in Rhesus Monkeys, The American Journal of Psychiatry, 121.

Mauck B. et Dehnhardt G., 1997, Mental rotation in a California sea lion, The Journal of Experimental Biology, 200, pp. 1309-1316.

Mauz I. et Rémy J., 2004, Biodiversités et agriculteurs des Alpes du Nord, Fourrages, 179.

Mauz I., 2002, Les conceptions de la juste place des animaux dans les Alpes françaises, Espaces et sociétés, 110-111.

Mauz I., 2005, Gens, cornes et crocs, Paris, Cemagref, Cirad, Ifremer, Inra.

Maynard Smith J., 1982, Evolution and the Theory of Games, Cambridge UP.

Maynard Smith J., 1989, Games, Sex and Evolution, London, Harvester and Wheatsheaf.

Mayr E., 1984, Populations, espèces et évolution, trad. franç., Paris, Hermann 1963.

Mc Farland D. (dir.), 1990, Dictionnaire du comportement animal, Paris, Robert Laffont, « Bouquins ». 
Mc Grew W., 1992, Chimpanzee Material Culture, Cambridge University Press.

Merleau-Ponty M., 1976, Phénoménologie de la perception (1945), Paris, Gallimard.

Merleau-Ponty M., 1977, la structure du comportement (1942), Paris, Puf.

Merleau-Ponty M., 1995, La Nature (Notes. Cours du Collège de France 1956-1957), Paris, Le Seuil.

Michoud L., 1906, La théorie de la personnalité morale et son application en droit français, LGDJ.

Micoud A., 1989, Les animaux dits «nuisibles » : essai sur l'évolution récente d'une notion, Rapport Sretie (Convention Sretie/Mere/88050), 51 p.

Moine I., 1997, Les choses hors du commerce, une approche de la personne humaine juridique, Paris, LGDJ.

Montagu A., 1996, The Elephant-Man. A Study in human dignity, New York, Arcadian House.

Morange M., 2005, Les secrets du vivant. Contre la pensée unique en biologie, Paris, La Découverte.

Morin E., Piattellini-Palmarini M., 1974, L'Unité de l'homme, t.1, Paris, Seuil.

Mounoud P. et Vinter A., 1981, La reconnaissance de son image chez l'enfant et l'animal, Paris, Delachaux et Niestlé.

Mouret S. et Porcher J., 2007, Les systèmes industriels porcins : la mort comme travail ordinaire, Natures, Sciences, Sociétés, 15, pp. 245-252.

Natale F. et Antinucci F., 1989, Stage 6 object-concept and representation, in : F. Antinucci(ed.), Cognitive structure and development in nonhuman primates, Hillsdale, Lawrence Erlbaum, pp. 97-112.

Natale F., Antinucci F., Spinozzi G. et Pòti P., 1986, Stage 6 object-concept in non human primate cognition : A comparison between gorilla (Gorilla gorilla) and Japanese macaque (Macaca fuscata), Journal of Comparative Psychology, 100, pp. 335-339.

Naucierus J., 1579, Chronica, éd. Cologne.

Nouët J.-C., 1988, Émergence des droits de l'animal, in : A. Couret et Fr. Oge (dir.), Homme, animal, société, t. II, Droit et animal, Toulouse, Presses de l'Institut d'Études politiques de Toulouse.

Novac M., Simund K., 1993, A Strategy of Win-Stay, Lose-Shift that Outperform Tit-for-Tat in the Prisoner's Dilemna Game, Nature, vol 364.

Nurock V., 2006, Origines et genèse du sens moral, Thèse de philosophie soutenue à Paris X Nanterre, $552 \mathrm{p}$.

Oé K., 1968, Une Affaire personnelle, Paris, Stock.

Pascal B., 1963, Préface sur le Traité du vide, in : Euvres complètes, éd. Lafuma, Paris, Seuil, L'Intégrale.

Pasqualini F., 1997, L'animal et la famille, D., Chron., p. 257. 
Patriquin M.L., Steyn M. et Loth S.R., 2005, Metric analysis of sex differences in South African black and white pelves, Forensic Science International, 147, pp. 119-217.

Patterson F. et Cohn R.H., 1994, Self-recognition and self-awareness in lowland gorillas in : S.T. Parker, R.W. Mitchell, et M.L. Boccia (eds.), Self-awareness in animals and humans, Cambridge, Cambridge University Press, pp. 273-290.

Perner J. et Wimmer H., 1985, John thinks that Mary thinks... : Attribution of secondorder beliefs by 5- to 10-year-old children, Journal of Experimental Child Psychology, 39, pp. 437-471.

Perner J., Leekam S.R. et Wimmer H., 1987, “Three-year-old's difficulty with false belief”, British Journal of Developmental Psychology, 5, pp. 125-137.

Plavcan J.M., Lokwood C.A., Kimbel W.H., Lague M.R., et Harmon E.H., 2005. Sexual dimorphism in Autralopithecus afarensis revisited: How strong is the case for a human-like pattern of dimorphism ?, Journal of Human Evolution, 48, pp. 313-320.

Porcher J., 2001, Éleveurs et animaux, réinventer le lien, Paris, Puf.

Porcher J., 2002, L'esprit du don, archaïsme ou modernité de l'élevage : éléments pour une réflexion sur la place des animaux d'élevage dans le lien social, Revue du Mauss, 20, Quelle autre mondialisation?, pp. 245-262.

Porcher J., 2006, Construction de l'insensibilité dans les productions animales, in : Michel Peroni et Jacques Roux (dir.), Sensibiliser, la sociologie dans le vif du monde, La Tour d'Aigues, Éditions de l'Aube.

Porcher J., 2007, Ne libérez pas les animaux !, Revue du Mauss, 29, premier semestre.

Porcher J., Le travail des femmes en porcheries industrielles : le prix de la reconnaissance, Ethnographiques. org, à paraître.

Porcher J., Cousson-Gélie F., Dantzer R., 2004, Affective components of the human-animal relationship in animal husbandry. Development and validation of a questionnaire. Psychological Reports, 95, pp. 275-290.

Povinelli D.J., Bering J.M. et Giambrone S., 2000, Toward a science of other minds : Escaping the argument by analogy, Cognitive Science, 24, pp. 509-541.

Povinelli D.J., Nelson K.E., et Boysen S.T., 1990, Inerences about guessing and knowing by chimpanzees (Pan troglodytes), Journal of Comparative Psychology, 104, pp. 203-210.

Povinelli D.J., Parks K.A. et Novak M.A., 1991, Do rhesus monkeys (Macaca mulatta) atribute knowledge and ignorance to others ?, Journal of Comparative Psychology, 105, pp. 318-325.

Povinelli D.J., Rulf A.B. et Bierschwale D.T., 1994, Absence of knowledge attribution and self-recognition in young chimpanzees (Pan troglodytes), Journal of Comparative Psychology, 108, pp. 74-80.

Premack D. et Woodruff G., 1978, Does the chimpanzee have a theory of mind?, The Behavioral and Brain Sciences, 3, pp. 615-636.

Preston S., De Waal F., 2002, Empathy: its Ultimate and Proximate Bases, Brain and Behavioural Science, 25. 
Proctor R.N., 2004, Three roots of human recency : molecular anthropology, the refigured Acheulan and the UNESCO response to Auschwitz, in: The Moral Authority of Nature, Chicago, The University of Chicago Press, L. Daston et F. Vidal, eds, pp. 466-490.

Proust J., 1997, Comment l'esprit vient aux bêtes, Paris, Gallimard.

Proust J., 2000, L'animal intentionnel, Terrain, 34, Les animaux pensent-ils ?

Proust J., 2003, Les animaux pensent-ils ?, Paris, Bayard.

Proust M., 1971, Jean Santeuil, Euvres complètes, Paris, Gallimard, « La Pléiade ».

Regan T., 1983, The Case for Animal Rights, Berkeley, University of California Press, London, Routedge § Kegan Paul.

Renaut A., 1986, Le système du droit. Philosophie et droit dans la pensée de Fichte, Paris, Puf.

Renck J.-L. et Servais V., 2002, L'éthologie, Histoire naturelle du comportement, Paris, Seuil.

Reno P.L., Meindl R.S., McCollum M.A. et Lovejoy C.O., 2003, Sexual dimorphism in : Australopithecus afarensis was similar to that of modern humans, Proceedings of the National Academy of Sciences USA, 100, pp. 9404-9409.

Rey A. (dir.), Dictionnaire historique de la langue française, 2000, Le Robert.

Ricœur P., 1950, Philosophie de la volonté, tome 1, le volontaire et l'involontaire, Paris, Aubier.

Ringel F. et Putman E., 1995, L'animal aimé par le droit, RRJ (Droit prospectif) 1995 (1).

Rodrigo P., 2004, La dynamique de l'hexis chez Aristote, Revue Alter, 12, L'habitude, pp. 11-26.

Rousseau J.J., 1969, Émile ou de l'éducation, livre III, Euvres complètes t. IV, Paris, Gallimard, «La Pléiade ».

Rousseau J.J., 1969, Quelle est l'origine de l'inégalité parmi les hommes et si elle est autorisée par la loi naturelle? Paris, Garnier.

Sacco R., 1995, Le droit muet, RTD civ. 1995.

Sahlins M., 1980, Critique de la sociobiologie. Aspects anthropologiques, trad. franç., Paris, Gallimard.

Schaeffer J.-M., 2007. La fin de l'exception humaine, Paris, Gallimard, « Essais ».

Schäppi R., 2002, La femme est le propre de l'homme. De l'éthologie animale à la nature humaine, trad. franç., Paris, Odile Jacob.

Scheler M., 1951, La Situation de l'homme dans le monde [1928], trad. franç., Paris, Aubier.

Schilcher F. von et Tennant N., 1984, Philosophy, Evolution and Human Nature, Londres, Routledge and Kegan Paul.

Schilder P., 1968, L'Image du corps, trad. franç. F. Gantheret et P. Truffert, Paris, Gallimard.

Schopenhauer A., 2004, Le monde comme volonté et comme représentation, trad. franç., Paris, Puf. 
Schouman M., Simard D., 2006, Sexualité, famille, procréation. Faut-il obéir à la nature?, Paris, Arnaud Franel éditions.

Serres M., 1985, Les cinq sens, Paris, Grasset.

Serres M., 1994. Éclaircissements, Entretiens avec Bruno Latour, Paris, Flammarion, Champs.

Shepard R. et Metzler J., 1971, Mental rotation of three-dimensional objects, Science, 171, pp. 701-703.

Sidgwick H., 1999, Methods of Ethics (1874) et préface à la première édition de Methods of Ethics, C. Audard (éd.), Anthologie historique et critique de l'utilitarisme, Tome II, trad. franç., Paris, Puf.

Simondon G., 2004, Deux leçons sur l'animal et l'homme, Paris, Ellipses, « Petite bibliothèque de philosophie ».

Singer P., 1993, La libération animale, trad. franç., Paris, Grasset.

Singer P., 1997, Questions d'éthique pratique, trad. franç., Paris, Bayard.

Sloderdijk P., 2003, Ni le soleil, ni la mort. Jeu de piste sous forme de dialogues avec Hans-Jürgen Henrichs, Paris, J.J. Pauvert.

Sloterdijk P., 2000, Règles pour le parc humain. Paris, Mille et une nuits.

Spinoza B., 1954, Léthique, De la servitude humaine, proposition VIII, Euvres complètes, trad. franç., Paris, Gallimard, « La Pléiade ».

Stevens J., Hauser M. D., 2004, Why be nice ? Psychological contraints on the evolution of cooperation, Trends of Cognitive Science, 8.

Straus E., 2000, Du sens des sens, trad. franç., Grenoble, Jérôme Million.

Stroobants M., 1978, Étude éthologique de certains aspects du comportements social des enfants à l'école maternelle, in D. Morris, Éthologie des primates, trad. franç., Bruxelles, éd. Complexe.

Suarez S.D. et Gallup G.G., 1981, Social responding to mirrors in rhesus macaques (Macaca mulatta): Effects of changing mirror location, American Journal of Primatology, 11, pp. 239-244.

Swartz K.B. et Evans S., 1991, Social and cognitive factors in chimpanzee and gorilla mirror behavior and self-recognition, in : Parker S.T., Mitchell R.W., et Boccia M.L. (eds.), Self-awareness in animals and humans, Cambridge University Press, pp. 189-206.

Terestchenko M., 2005, Un si fragile vernis d'humanité. Banalité du mal, banalité du bien, Paris, La Découverte. Mauss.

Terré F., Fenouillet D., 2005, Droit civil. Les personnes, la famille, les incapacités, Paris, Dalloz, collection Précis - Droit privé, $7^{\mathrm{e}}$ éd., 13.

Thinès G., 1980, Phénoménologie et science du comportement [1977], trad. franç., Liège, Pierre Mardaga éditeur.

Thomas Y., 1998, Le sujet de droit, la personne et la nature. Sur la critique contemporaine du sujet de droit, Le débat, 100, mai-août. 
Tinbergen N., 1967, La Vie sociale des animaux, trad. franç., Paris, Payot.

Tinbergen N., 1980, L'Étude de l'instinct, trad. franç., Paris, Payot.

Tinland F., 2003, L’homme sauvage, Paris, Payot, 1968, réed., Paris, L'Harmattan.

Tisseron S., 1996, Secrets de Famille, Mode d'emploi, Paris, Ramsay.

Tomasello M. et J. Call J., 1997, Primate cognition, New York et Oxford, Oxford University Press.

Trivers R., 1971, The evolution of reciprocal altruism, Quaterly Review of Biology, 46.

Uexküll J. von, 2004, Mondes animaux et monde humain suivi de Théorie de la signification [1934], trad., franç., Paris, Denoël.

Vauclair J., 1984, Phylogenetic approach to object manipulation in human and ape infants, Human Development, 27, pp. 321-328.

Vauclair J., 1995, L'intelligence de l'animal. Paris, Éditions du Seuil, « Points/Sciences ».

Vauclair J., 2004, Développement du jeune enfant : Motricité, perception, cognition, Paris, Belin.

Vauclair J., Fagot J. et Hopkins W.D., 1993, Rotation of mental images in baboons when the visual input is directed to the left cerebral hemisphere, Psychological Science, 4, pp. 99-103.

Visalberghi E., 1986, Aspects of space representation in an infant gorilla in : D.M. Taub et F.A. King (eds.), Current perspectives in primate social dynamics, New York, Van Nostrand Reinhold, pp. 445-452.

Weinstein B., 1945, The evolution of intelligent behavior in rhesus monkeys, Genetic Psychology Monographs, 31, pp. 2-48.

Welsch P. c/ Préfet de la Moselle, 2002, Dictionnaire Permanent Bioéthique et Biotechnologies, Bulletin 114, 26 avril 2002, 9.

Whiten A., Goodal J., McGrew W. C. et al., 1999, Cultures in chimpanzees, Nature, 399, pp. 682-685.

Wilson E. O., 1987, La sociobiologie (La nouvelle synthèse) [1975], trad. franç., Monaco, Éd. du Rocher.

Wittgenstein L., 1961, Les investigations philosophiques, trad. franç., reproduites d'après le Tractatus logico-philosophicus, Paris, Gallimard.

Wolff-Quenot M.-J., 1996, Des Monstres aux mythes, Paris, Guy Trédaniel éditeur.

Wrangham R., De Waal F. et Mc Grew W., 1994, Chimpanzee Cultures, Harvard University Press.

Wright R., 1985, The Moral Animal, Evolutionary Psychology of Everyday Life, New York, Random House, (trad. franç. L'animal moral, 1994, Paris, Michalon).

Wuketis F.M., 1990, Evolutionary Epistemology and its Implications for Humankind, New York. State University of New York Press.

Zazzo R., 1975, Des jumeaux devant le miroir. Questions de méthode, Journal de Psychologie, 4, pp. 389-413. 


\section{Liste des auteurs}

\section{Ancet Pierre}

UFR de Lettre et Philosophie, Centre Gaston Bachelard,

UMR-CNRS 5605, Université de Bourgogne, 2 bd Gabriel, 21000 Dijon

pierre.ancet@u-bourgogne.fr

Brun Bernard

Université de Provence

18 rue César Aleman, 13007 Marseille

bdbrun@numericable.fr

Burgat Florence

INRA, unité TSV

63 bd de Brandebourg 94205 Ivry-sur-Seine

burgat.florence@wandoo.fr

Camos Valérie

Institut Universitaire de France

UFR de Sciences Humaines, Département de Psychologie, LEAD-CNRS UMR 5022, Université de Bourgogne, Pôle AAFE, Esplanade Erasme, BP 26513, 21065 Dijon valerie.camos@u-bourgogne.fr

Cézilly Frank

Institut Buffon,

UFR de Science Vie, Département Ecologie, UMR-CNRS Biogéosciences, Université de Bourgogne, 6 bd Gabriel, 21000 Dijon

frank.cezilly@u-bourgogne.fr

Desmoulin Sonia

UMR de Droit comparé de Paris, Equipe CRDST,

Université Paris1 Panthéon-Sorbonne, 9 rue Malher, 75004 Paris

sonia.desmoulin@laposte.net 


\section{Granjou Céline}

Cemagref, UR DTM

2 rue de la Papeterie, BP 76, 38402 Saint-Martin-d'Hères Cedex

celine.granjou@grenoble.cemagref.fr

Guenancia Pierre

UFR de Lettre et Philosophie, Département de Philosophie, Centre Gaston Bachelard, UMR-CNRS 5605, Université de Bourgogne, 2 bd Gabriel, 21000 Dijon pierre.guenacia@u-bourgogne.fr

Guichet Jean-Luc

Collège international de philosophie et Centre Gaston Bachelard,

UMR-CNRS 5605, Université de Bourgogne, 2 bd Gabriel, 21000 Dijon

Larrère Catherine

UFR de philosophie, Université Paris 1, Panthéon-Sorbonne,

17 rue de la Sorbonne, 75231 Paris cedex 05

c.larrere@wanadoo.fr

Larrère Raphaël

INRA, unité TSV

63 bd de Brandebourg, 94205 Ivry-sur-Seine

larrere@ivry.inra.fr

Mauz Isabelle

Cemagref, UR DTM

2 rue de la Papeterie, BP 76, 38402 Saint-Martin-d'Hères Cedex

isabelle.mauz@grenoble.cemagref.fr

Pierron Jean-Philippe

Faculté de Philosophie, Université Lyon 3, Jean Moulin

7 rue de Chevreuil, 69007 Lyon

kitoup@club-internet.fr

Porcher Jocelyne

INRA-SADAPT,

16 rue Claude Bernard 75231 Paris Cedex 05

jo.porcher@wanadoo.fr

Rochette Luc

Laboratoire de Physiopathologie et de Pharmacologie Cardiovasculaire

Faculté de Médecine et de Pharmacie, Université de Bourgogne, 7 bd Jeanne d'Arc, BP 87900, 21079 Dijon

luc.rochette@u-bourgogne.fr

Sylvestre Jean-Pierre

UFR de Sciences Humaines, Département de Sociologie, UMR-CNRS 5605, Université de Bourgogne, Pôle AAFE, Esplanade Erasme, BP 26513, 21065 Dijon

Jean-pierre.sylvestre@u-bourgogne.fr

Vauclair Jacques

Université de Provence, Centre Psyclé

29 avenue Schuman, 13621 Aix-en-Provence Cedex 1

Jacques.vauclair@univ-provence.fr 

Formaté typographiquement par Desk :

0243012211 
Deux grands types de critiques convergent aujourd'hui pour remettre en question la thèse de la singularité radicale de I'homme. Au plan scientifique d'abord, de nombreux primatologues tendent à mettre en évidence que, du point de vue de l'identité psychologique comme de celui des performances cognitives, la différence entre les grands singes et l'homme ne serait pas de nature mais seulement de degrés.

D'une manière générale, la biologie de l'évolution ne nie pas l'existence de différences majeures entre l'homme et les animaux, même supérieurs, mais elle les réintroduit dans un paradigme continuiste, évolutionniste et non anthropocentré. La distinction entre l'homme et les autres animaux peut être maintenue, non pas tant comme une différence entre nature et culture, mais comme un ensemble de différences dans la nature.

Au plan éthique, des formes inédites d'appropriation des animaux vivants par l'homme ont conduit à instrumentaliser ces derniers. À cela s'ajoutent les menaces pesant sur les espèces sauvages en raison du développement des sociétés industrielles. Certains en viennent ainsi à admettre une véritable solidarité entre les formes de vie humaines et animales permettant de reconsidérer en profondeur les normes morales ou juridiques qui régentent et régulent l'ensemble des relations entre humains et non-humains.

Ce recueil n'a pas pour vocation d'apporter une réponse univoque à l'ensemble des problèmes posés. Il réunit des textes à caractère spéculatif et des observations plus empiriques. Écrit par des philosophes, des éthologues, des sociologues et des biologistes, il s'adresse aux étudiants et spécialistes des disciplines abordées ainsi qu'à tous les lecteurs que ce sujet, actuellement en débat, intéresse.

Professeur de psychologie à l'Université de Bourgogne et membre de l'Institut universitaire de France, Valérie Camos dirige l'équipe Développement au sein du laboratoire d'étude de l'apprentissage et du développement au CNRS.

Professeur d'écologie comportementale à l'Université de Bourgogne, Frank Cézilly est directeur de I'Institut Buffon et directeur adjoint de l'unité Biogéosciences du CNRS.

Professeur d'histoire de la philosophie moderne à l'Université de Bourgogne,

Pierre Guenancia est directeur du Centre Gaston Bachelard de recherche sur l'imaginaire et la rationalité au CNRS.

Jean-Pierre Sylvestre, est professeur de sociologie à l'Université de Bourgogne et chercheur dans I'unité mixte de recherches Georges Chevrier au CNRS.

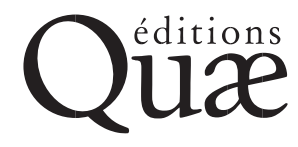

Éditions Cemagref, Cirad, Ifremer, Inra www.quae.com

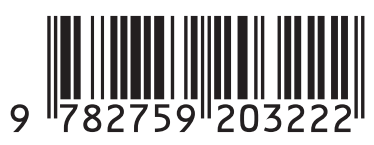

ISSN : 1773-7923

Réf. : 02137 